\title{
Coupling Numerical Simulation and Pattern Recognition to Model Production and Evaluate Carbon Dioxide Injection in Shale Gas Reservoir
}

Amirmasoud Kalantari-Dahaghi

West Virginia University

Follow this and additional works at: https://researchrepository.wvu.edu/etd

Part of the Artificial Intelligence and Robotics Commons, Hydraulic Engineering Commons, and the Mining Engineering Commons

\footnotetext{
Recommended Citation

Kalantari-Dahaghi, Amirmasoud, "Coupling Numerical Simulation and Pattern Recognition to Model Production and Evaluate Carbon Dioxide Injection in Shale Gas Reservoir" (2013). Graduate Theses, Dissertations, and Problem Reports. 8164.

https://researchrepository.wvu.edu/etd/8164

This Dissertation is protected by copyright and/or related rights. It has been brought to you by the The Research Repository @ WVU with permission from the rights-holder(s). You are free to use this Dissertation in any way that is permitted by the copyright and related rights legislation that applies to your use. For other uses you must obtain permission from the rights-holder(s) directly, unless additional rights are indicated by a Creative Commons license in the record and/ or on the work itself. This Dissertation has been accepted for inclusion in WVU Graduate Theses, Dissertations, and Problem Reports collection by an authorized administrator of The Research Repository @ WVU. For more information, please contact researchrepository@mail.wvu.edu.
} 
Coupling Numerical Simulation and Pattern Recognition to Model Production and Evaluate $\mathrm{CO}_{2}$ Injection in Shale Gas Reservoir

By

Amirmasoud Kalantari-Dahaghi

Dissertation submitted to the

Benjamin M. Statler College of Engineering and Mineral Resources

at West Virginia University

in partial fulfillment of the requirements for the degree of

Doctor of Philosophy

in

Petroleum and Natural Gas Engineering

Approved by

Shahab D. Mohaghegh, PhD., Chair

Samuel Ameri, Department Chair

KashyAminian, PhD

Timothy Carr, PhD

Grant Bromhal, Ph.D

Petroleum and Natural Gas Engineering Department

Morgantown, West Virginia

2013

Keywords: Shale Gas, Numerical Simulation, Hydraulic Fracture Cluster, Data-driven Proxy Model, Pattern Recognition, Artificial Intelligence, $\mathrm{CO}_{2}$ Sequestration, Enhanced Gas Recovery

(C) Copyright 2013 Amirmasoud Kalantari-Dahaghi 


\section{ABSTRACT \\ Coupling Numerical Simulation and Pattern Recognition to Model Production and Evaluate $\mathrm{CO}_{2}$ Injection in Shale Gas Reservoir}

\section{Amirmasoud Kalantrai-Dahaghi}

Massive multi-cluster, multi-stage hydraulic fractures have significantly increased the complexity of the flow behavior in shale. This has translated into multiple challenges in the modeling of production from shale wells.

Most commonly used numerical techniques for modeling production from shale wells are Explicit Hydraulic Fracture (EHF) and Stimulated Reservoir Volume (SRV). Model setup for the EHF technique is long and laborious and its implementation is computationally expensive, such that it becomes impractical to model beyond a single pad. On the other hand, identifying the extent and conductivity of SRV is a challenging proposition. SRV technique is commonly used to simplify the modeling and the history matching process.

In this dissertation, an integrated workflow, which demonstrates a quantitative platform to model shale gas production through capturing the essential characteristics of shale gas reservoirs, is developed. A dual porosity/ compositional simulation model with explicit hydraulic fractures is developed for a pad with six horizontal laterals and 169 clusters of hydraulic fractures in the Marcellus shale reservoir. This pad is history matched using three years of production history.

The history-matched model is used to develop Next-generation shale proxy model (data-driven shale proxy model) at the hydraulic fracture cluster level, using pattern recognition technology. Data-driven shale proxy model provides highly accurate simulation results for the methane production in a second, thus making a comprehensive analysis of production from shale a practical and feasible option.

The history-matched and depleted Marcellus shale gas reservoir simulation model is used to perform a feasibility study to evaluate $\mathrm{CO}_{2}$ injection process for the purpose of production enhancement and $\mathrm{CO}_{2}$ storage by coupling numerical simulation and pattern recognition capabilities of Artificial Intelligence.

Data-driven shale proxy model for $\mathrm{CO}_{2}$ Enhance Gas Recovery and Storage $\left(\mathrm{CO}_{2}\right.$-EGR\&S) is developed, which is capable of accurately replicating the generated injection and production profiles from the numerical simulation model for each cluster/stage and horizontal lateral.

Coupled use of the deterministic reservoir model with Data-driven shale proxy model is served as a novel screening and optimization tool in evaluating the viability of residual gas recovery and $\mathrm{CO}_{2}$ storage in depleted (or near-depleted) shale gas formations. It allows running the model in real time and making the uncertainty quantification possible for $\mathrm{CO}_{2}$-EGR\&S process. 


\section{DEDICATION}

This dissertation is dedicated to my family specially my Dad

For unconditional love, unwavering support and endless encouragement. 


\section{ACKNOWLEDGEMENT}

My sincere appreciation and special thanks goes to my advisor, Dr. Shahab D. Mohaghegh, who has been a dedicated mentor, a knowledgeable advisor and a great friend. Without his guidance and support, this journey would not be possible.

I would also like to thank Professor Sam Ameri, the department chair, for his blessings during this long process; he has always been there for me. I would like to offer my appreciation to my committee members specially Dr.Kashy Aminian for all of his support and also Dr.Timothy Carr, Dr. Grant Bromhal; many thanks for your interest in my work, supportive inputs and thought-provoking suggestions.

I also would express my gratitude to my fellows in Petroleum Engineering and Analytics Research Lab. (Jalal Jalali, Cesar Cilva, Camilo Calderon, Yorgi Gomez, Qin He, Daniel and David Moreno, Faegheh Javadi, Sajad Omidvar) for their assistance over the past five years. I am also grateful for my classmates in Petroleum and Natural Gas Engineering Department of WVU and my friends in all over the world for their friendship, positive inspirational messages and encouragements. My sincere appreciation is extended to my best friend, Soodabeh Esmaili, who has been always with me during all steps of my life during the past three years.

I also want to thank Intelligent Solutions Inc. and Schlumberger (Abingdon Technology Center and SISHouston) for providing the software packages and technical support. I would like to thank U.S. Department of Energy-National Energy Technology Laboratory for providing financial assistance.

I want to acknowledge the help and support that I received during these years from Ms. Beverly Matheny, Dr. Melanie Cook, Dr. Constinia Charbonnette and Dr.Grace Atebe.

Finally, my deepest gratefulness goes to my family for their unflagging love and unconditional support throughout my life and my studies.

Above all, utmost appreciation to the Almighty God for the divine intervention in this academic endeavor; I would not have come this far without his blessing. 


\section{Table of Content}

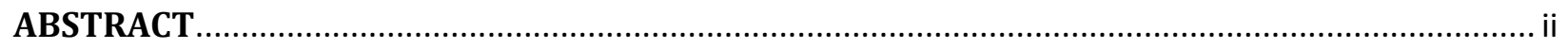

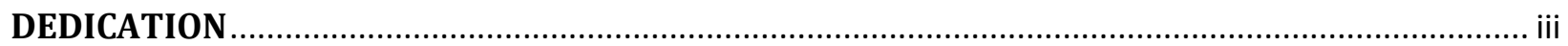

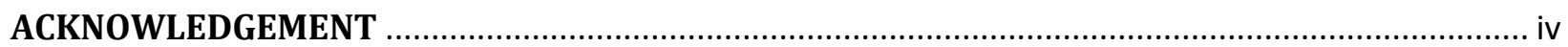

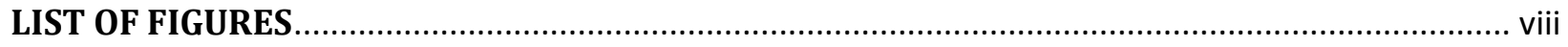

LIST OF TABLES ……………………………………………………………………………...iii

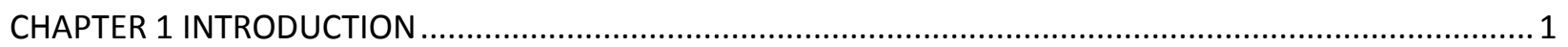

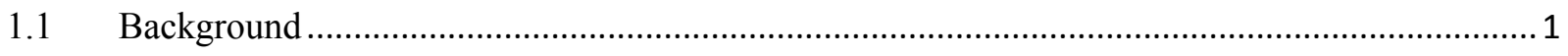

$1.2 \quad$ Problem Statement and Dissertation Contributions ................................................................. 4

1.3 Dissertation Organization ……………………………………………………………....

CHAPTER 2 LITERATURE REVIEW ...................................................................................... 9

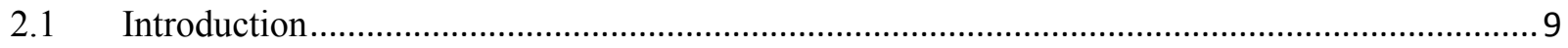

2.2 Numerical Methods for Shale Gas Reservoir....................................................................

2.3 $\quad \mathrm{CO}_{2}$-Enhanced Gas Recovery and Storage in Shale Reservoir.................................................15

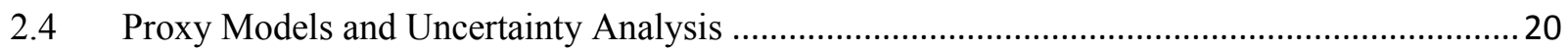

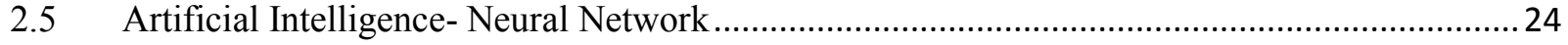

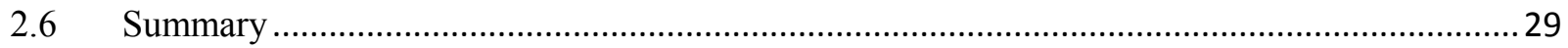

CHAPTER 3 SHALE GAS NUMERICAL SIMULATION DEVELOPMENT- INTEGRATED WORKFLOW.................30

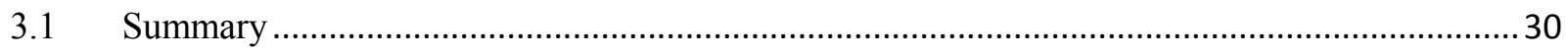

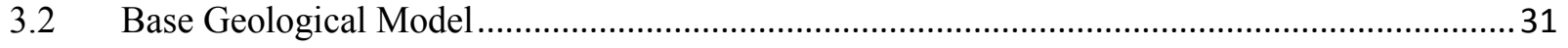

3.2.1 Data collection .......................................................................................................... 31

3.2.2 Structural Modeling …………………………………………………………….... 32

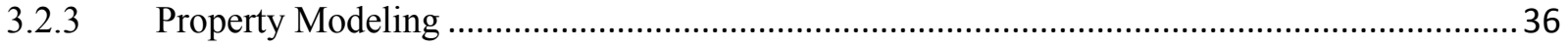

3.2.4 Natural Fracture Modeling -DFN Generation .................................................................... 39

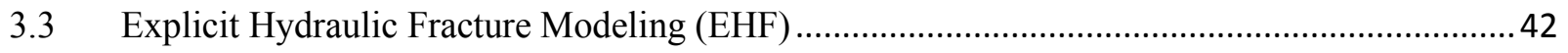

3.4 History Matching and Forecasting Production ……………………………………………..... 46

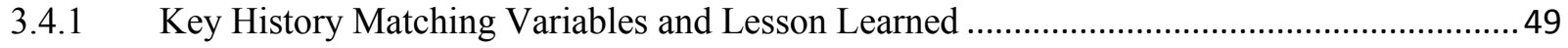

3.5 Summary and Conclusions...............................................................................................

CHAPTER 4 -DATA DRIVEN MODEL, A NEXT-GENERATION SHALE PROXY AT HYDRAULIC FRACTURE

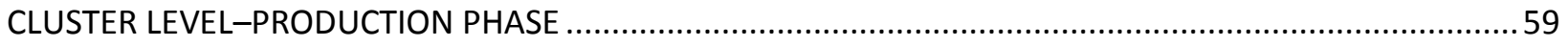

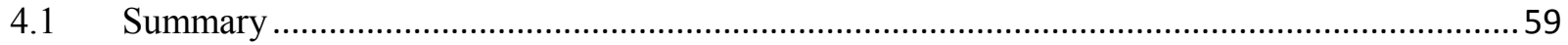

4.2 Data-driven Shale Proxy Model...…………………………………………………..... 59 


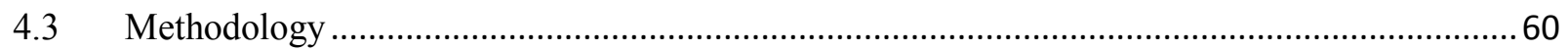

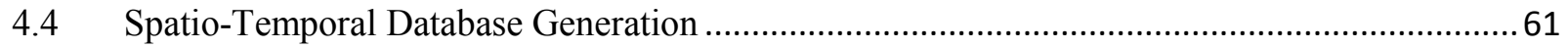

4.5 Data-Driven Proxy Model Development for Different Production Time Resolutions ...............66

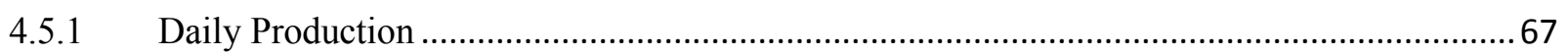

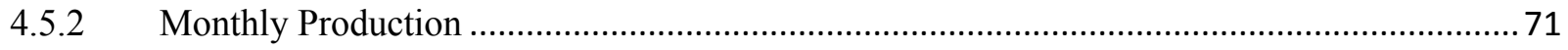

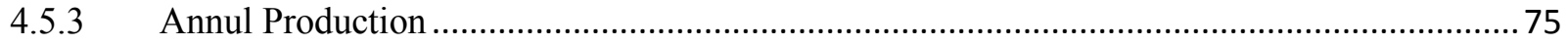

4.6 Data-Driven Proxy Models Validation by Blind Case .......................................................... 79

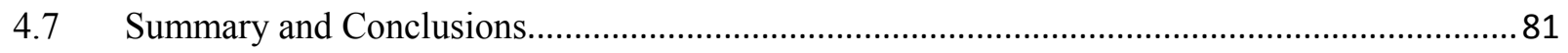

CHAPTER 5 -TECHNICAL ASPECTS OF $\mathrm{CO}_{2}$-ENHANCED GAS RECOVERY AND STORAGE (CO ${ }_{2}$-EGR\&S) IN

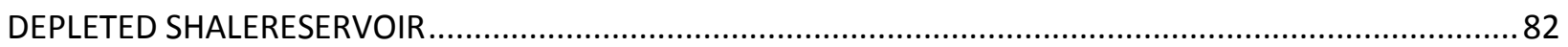

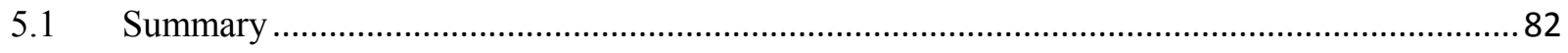

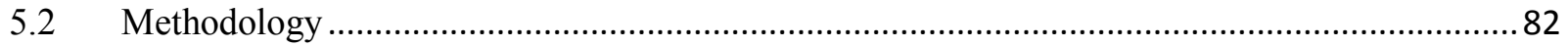

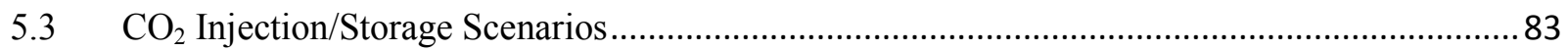

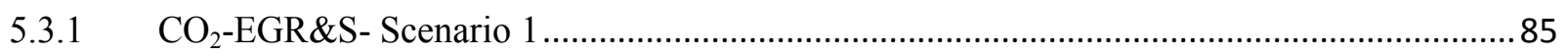

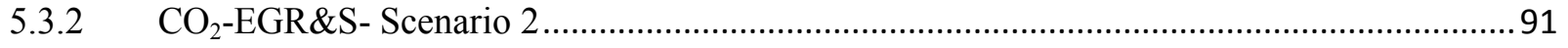

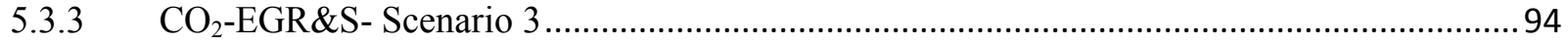

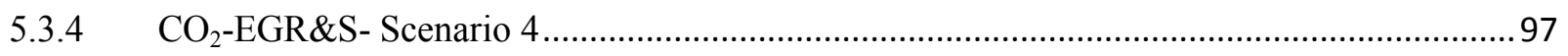

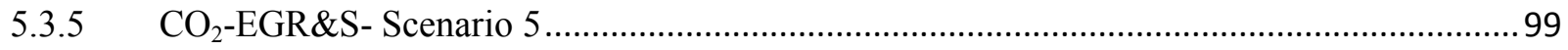

5.4 Effect of Sorption Isotherm on $\mathrm{CO}_{2}$-EGR\&S Process ....................................................... 105

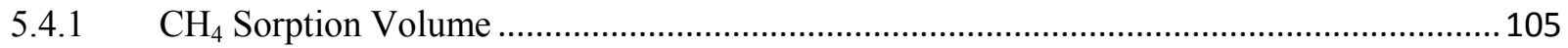

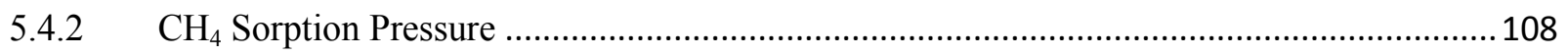

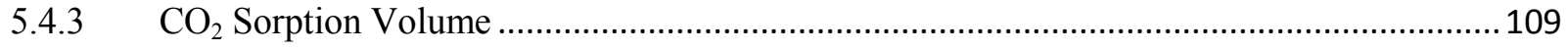

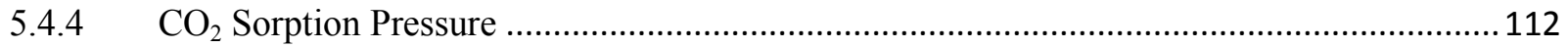

5.4.5 Comparison of the impact of $\mathrm{CO}_{2}$ and $\mathrm{CH}_{4}$ Isotherms on Methane Recovery, $\mathrm{CO}_{2}$ injection

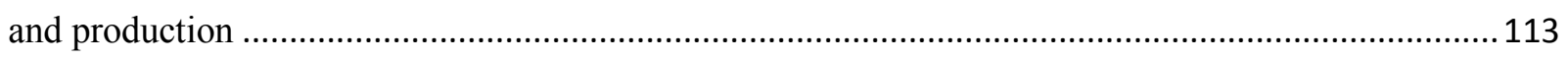

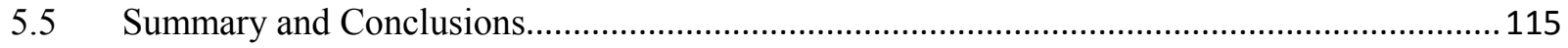

CHAPTER 6 - DATA-DRIVEN SHALE PROXY MODEL AT HYDRAULIC FRACTURE CLUSTER LEVEL -

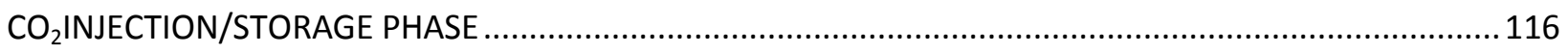

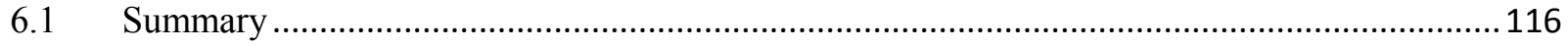

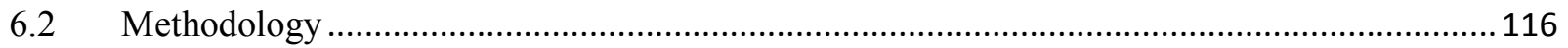

6.3 Spatio-Temporal Database Generation ....................................................................... 118

6.4 Data-Driven $\mathrm{CO}_{2}$-EGR\&S Proxy Development for $\mathrm{CH}_{4}$ Production Rate, $\mathrm{CO}_{2}$ Injection Rate and $\mathrm{CO}_{2}$ Breakthrough 
6.4.1 $\quad \mathrm{CH}_{4}$ Production Profile Predication .......................................................................... 120

6.4.2 $\quad \mathrm{CO}_{2}$ Injection Profile Predication.............................................................................. 125

6.4.3 $\quad \mathrm{CO}_{2}$ Breakthrough Time and Production Profile Predication ............................................. 129

6.5 Data-driven $\mathrm{CO}_{2}$-EGR\&S Proxy Model Validation by Blind Cases .....................................135

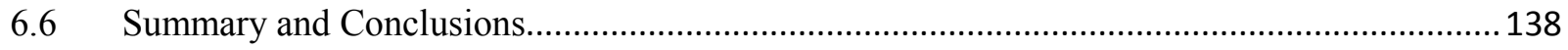

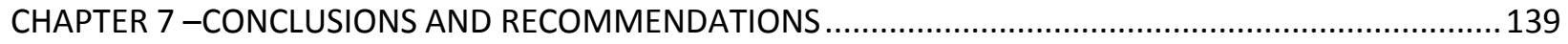

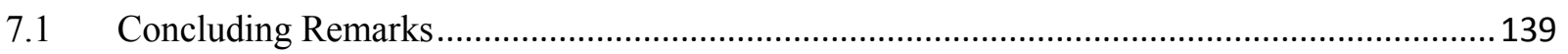

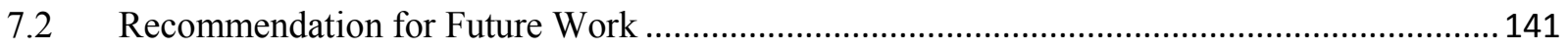

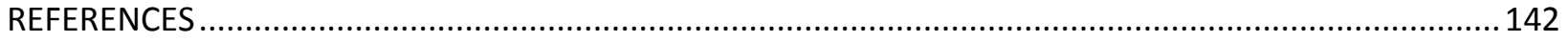

APPENDIX A -COMPARISON OF GAS PRODUCTION PROFILE FROM SIMULATOR AND DATA-DRIVEN PROXY MODEL FOR DIFFERENT TIME RESOLUTIONS (DAILY, MONTHLY \& ANNUAL)-PRODUCTION PHASE

APPENDIX B-COMPARISON OF $\mathrm{CH}_{4}, \mathrm{CO}_{2}$ PRODUCTION AND $\mathrm{CO}_{2}$ INJECTION RATES GENEARTED BY DATADRIVEN $\mathrm{CO}_{2}$-EGR\&SPROXY MODELS WITH THE NUMERICAL SIMULATION RESULTS 162 


\section{LIST OF FIGURES}

Figure 1-1.Map of shale gas plays and related basins in the U.S. lower 48 states. Sources: (U.S. Census Bureau, 2010; U.S. Energy Information Administration (EIA), 2011a, 2011b) ....

Figure 1-2.Map of Marcellus shale play, Marcellus shale producing wells and $\mathrm{CO}_{2}$ emitters, Sources: (Gurney et al., 2009; NPS, 2009; U.S. Census Bureau, 2010; EIA, 2011b; EPA, 2012a; Zagorski et al., 2011)

Figure 2-1.Dual porosity numerical and conceptual model (Kalantari-Dahaghi 2011) ........................... 10 Figure 2-2.Realization of shale-gas reservoir sample with components in series. The arrows indicate direction of gas flow (Hudson 2011) ................................................................................................. 11 Figure 2-3. Comparison of SRV and normalized gas production from each stage and Map View of

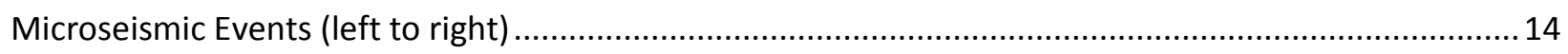

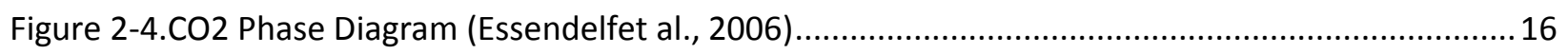

Figure 2-5.Artificial Neuron configuration (Barber 2007) .................................................................... 24

Figure 2-6.Three-layered neural network architecture (Kunzle 2003) ................................................. 25

Figure 3-1-Marcellus shale geomodeling to simulation workflow ....................................................... 30

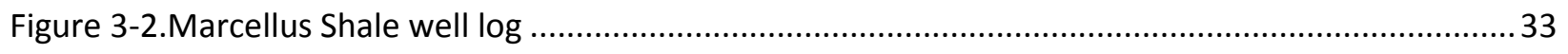

Figure 3-3.2D and 3D representation of Five cross-sections across the reservoir (Left to right) ............... 34

Figure 3-4.Cross Section /Top picking example in one of the cross sections .......................................... 34

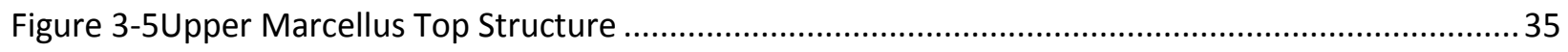

Figure 3-6.Distribution of 76 wells in the reservoir and reservoir boundary .......................................... 35

Figure 3-7.3D Grid skeleton representation for 1.16 million grid blocks ............................................... 36

Figure 3-8.Distribution of Matrix porosity, Net to Gross thickness and Matrix permeability in Upper and

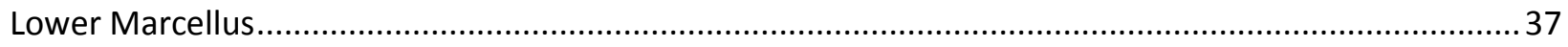

Figure 3-10. Distribution of Min. Horizontal stress gradient, Young's modulus and Poisson's ratio in

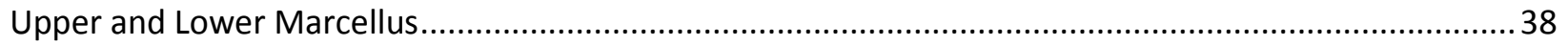

Figure 3-11.3D display of Dip angle point data for one of the wells. ..................................................... 40

Figure 3-12. FMI log example for one of the wells and fracture point data illustration on stereonet for all

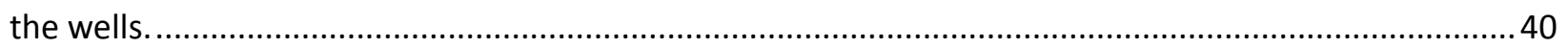

Figure 3-13. Dip angle distribution in generated Discrete Fracture Network (DFN) .............................. 41

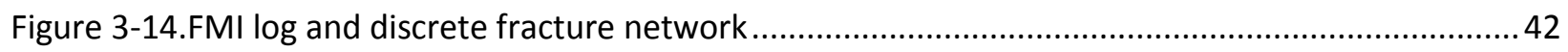

Figure 3-15.2D representation of generated hydraulic fracture with stress and width profile ................. 44

Figure 3-16-Local grid refinement for all the wells and the location and configuration of WVU pad ....... 45

Figure 3-17.3D LGR representation and distribution of hydraulic fractures conductivity ....................... 45

Figure 3-18. WVU Pad configuration and corresponding horizontal laterals ........................................ 46

Figure 3-19. Measured daily gas production and bottomhole pressure for all the wells in WVU Pad (Top to bottom relatively) ................................................................................................................. 47

Figure 3-20.An integrated workflow flowchart illustrating all steps from base model development to

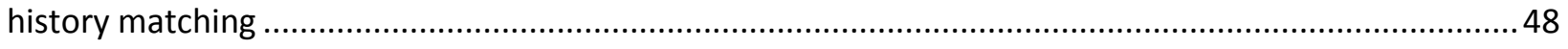

Figure 3-21Comparison of actual daily gas rate data points with the simulation results from Eclipse for

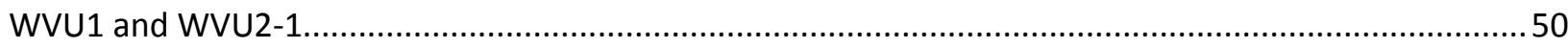


Figure 3-22.Comparison of actual daily gas rate data points with the simulation results from Eclipse for WVU3-1 and WVU3-3

Figure 3-23.Comparison of actual daily gas rate data points with the simulation results from Eclipse for WVU3-2 and WVU2-2

Figure 3-24.Comparison of actual daily gas rate data points with the simulation results from Eclipse for entire WVU pad

Figure 3-25. Gas production rate and bottomhole pressure changes for most of the wells after installing tubing. 54

Figure 3-26. 3D representation of hydraulic fracture conductivity for 169 clusters (Min. value=0.5 and Max.=5.4 md-ft) .55

Figure 3-27.Natural fracture permeability distribution in UM and LM from left to right (Min. value $=0.001$ and Max. $=0.01 \mathrm{md}$ ) .55

Figure 3-28.Matrix permeability distribution in UM and LM from left to right (Min. value $=0.0001$ and Max. $=0.001 \mathrm{md}$ ). 55

Figure 3-29. Matrix porosity distribution in UM and LM from left to right (Min. value=0.05 and Max. $=0.135 \mathrm{md}$ ) .56

Figure 3-30. NTG distribution in UM and LM from left to right (Min. value $=0.75$ and Max. $=0.98 \mathrm{md}$ ) ....56

Figure 3-31. Forecasting production behavior foal I WBVU pad's laterals

Figure 3-32.Pressure distribution in matrix after 6 month, 1 year, 3,5,10,20,30,60 and 90 years of production. .58

Figure 4-1. Data-driven Shale proxy model development workflow. .61

Figure 4-2. Ten different shale regions with unique sorption features .................................................. 63

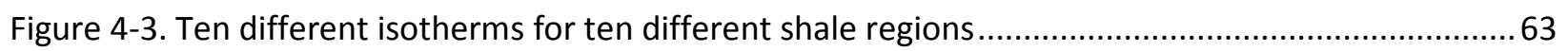

Figure 4-4. Different flowing bottomhole pressure profiles............................................................... 64

Figure 4-5.Tier system and cluster type definition (from left to right) ..................................................65

Figure 4-6. Illustration of generated production profile by Data-driven proxy model for each cluster and the corresponding lateral 66

Figure 4-7. Key performance indicators based on the daily database for the first two months of production. .68

Figure 4-8. NN training, calibration and verification results (From left to right relatively)- Daily proxy model .70

Figure 4-9. Comparison of gas production profile from simulator and Data-driven proxy model for some of the clusters 70 Figure 4-10. Comparison of gas production profile from simulator and Data-driven proxy model for some of the laterals....

Figure 4-11. Key performance indicators based on the monthly database for the first five years of production.

Figure 4-12.NN training, calibration and verification results (From left to right relatively)-Monthly proxy model......

Figure 4-13. Comparison of gas production profile from simulator and Data-driven proxy model for some of the laterals. .74 
Figure 4-14. Comparison of gas production profile from simulator and Data-driven proxy model for some of the clusters 75

Figure 4-15. Key performance indicators based on the monthly database for 100 years of production .. 76 Figure 4-16. NN training, calibration and verification results (From left to right relatively)-Annual proxy model. .77

Figure 4-17. Comparison of gas production profile from simulator and Data-driven proxy model for some of the clusters

Figure 4-18. Comparison of gas production profile from simulator and Data-driven proxy model for some of the laterals.

Figure 4-19. Combined monthly and annual proxies- Annual gas rate production for four clusters located

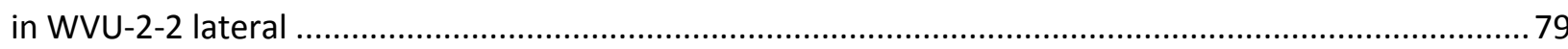

Figure 4-20. Combined monthly and annual proxies- Annual gas rate production WVU-2-2 lateral......... 79 Figure 4-21. Data-driven proxy models validation using blind run for WVU1 for different time resolutions

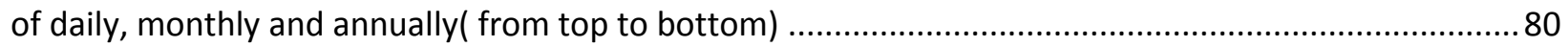

Figure 5-1. CO2-Enhanced Gas Recovery and Storage Workflow......................................................... 82

Figure 5-2.Highlighting Distance between adjacent laterals in part of WVU pad ................................... 83

Figure 5-3. Distribution of distances between lateral for Marcellus shale asset (Total number of 135

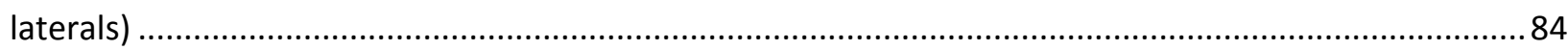

Figure 5-4. Schematics of five different injection scenarios for CO2 -EGR \&S process ............................ 85

Figure 5-5. $\mathrm{CO} 2$ injection rate profile and Comparison of $\mathrm{CH} 4$ cumulative production in base case and

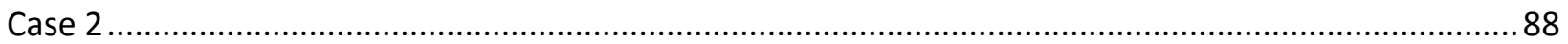

Figure 5-6. Methane molar density changes in monitoring wells in the scenario1- Case2 ...................... 89

Figure 5-7.Carbon dioxide molar density changes in monitoring wells in the scenario1- Case2 ............... 89

Figure 5-8. Well block pressure profile for all the wells during history matching, depletion and injection

Figure 5-9. $\mathrm{CO} 2$ injection rate profile and Comparison of $\mathrm{CH} 4$ cumulative production in base case and Case 2 92

Figure 5-10.Well block pressure profile for all the wells during history matching, depletion and injection

Figure 5-11.Methane and Carbon dioxide molar density changes in monitoring wells in scenario 2-Case2 (from top to bottom)

Figure 5-12.CO2 injection rate profile and Comparison of $\mathrm{CH} 4$ cumulative production in base case and Case 3

Figure 5-13.Methane and Carbon dioxide molar density changes in monitoring wells in scenario 3-Case2

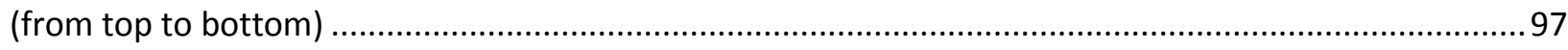

Figure 5-14-Production and injection wells schematic in Scenario 4 ......................................................... 98

Figure 5-15.CO2 injection rate profile and Comparison of $\mathrm{CH}_{4}$ cumulative production in base case and Case 1 101

Figure 5-16.CO2 injection rate profile and Comparison of $\mathrm{CH} 4$ cumulative production in base case and Case 2 102

Figure 5-17. Molar density of $\mathrm{CO} 2$ after $1,3,5,10,20$ and 50 years of injection.................................. 103

Figure 5-18. Molar density of Methane after 1,3,5,10,20 and 50 years of injection............................... 104 
Figure 5-19.Effect of different methane Langmuir volumes on $\mathrm{CH} 4$ cum production ............................106

Figure 5-20.Effect of different methane Langmuir volumes on $\mathrm{CO} 2$ injectivity ....................................... 106

Figure 5-21.Effect of different methane Langmuir volumes on $\mathrm{CO} 2$ breakthrough................................107

Figure 5-22.Effect of different methane Langmuir pressures on $\mathrm{CH} 4$ cum production .......................... 108

Figure 5-23.Effect of different methane Langmuir pressures on CO2 injectivity .................................. 109

Figure 5-24.Effect of different methane Langmuir pressures on CO2 breakthrough.............................110

Figure 5-25.Effect of different Carbone dioxide Langmuir volume on $\mathrm{CO} 2$ injectivity........................... 111

Figure 5-26.Effect of different carbon dioxide Langmuir volume on $\mathrm{CO} 2$ breakthrough........................111

Figure 5-27.Effect of different Carbone dioxide Langmuir pressure on $\mathrm{CO} 2$ injectivity...........................112

Figure 5-28.Effect of different carbon dioxide Langmuir pressure on $\mathrm{CO} 2$ breakthrough....................... 113

Figure 5-29. Impact of increasing $\mathrm{CH} 4$ and $\mathrm{CO} 2$ isotherms by $50 \%$ on total methane recovery.............. 114

Figure 5-30. Impact of increasing $\mathrm{CH} 4$ and $\mathrm{CO} 2$ isotherms by $50 \%$ on total injected $\mathrm{CO} 2$.....................114

Figure 5-31. Impact of increasing $\mathrm{CH} 4$ and $\mathrm{CO} 2$ isotherms by $50 \%$ on $\mathrm{CO} 2$ breakthrough ..................... 115

Figure 6-1. CO2-EGR\&SData-driven Shale proxy model development workflow..................................117

Figure 6-2. $\mathrm{CO}_{2}$-EGR\&S Process Simulation Cases (Producer and Injector patterns) ..............................118

Figure 6-3.Key performance indicators results based on the database for CO2-EGR\&S-Methane production rate (Mscf/year)

Figure 6-4. NN training, calibration and verification results (From left to right relatively) - $\mathrm{CH} 4$ Production (Mscf/year).

Figure 6-5.Comparison of $\mathrm{CH} 4$ production profile (Mscf/year) from simulator and CO2-EGR\&S Datadriven proxy model for some of the clusters (Cases1, 2 and 3) ...................................................... 123

Figure 6-6.Comparison of $\mathrm{CH} 4$ production profile (Mscf/year) from simulator and CO2-EGR\&S Datadriven proxy model for some of the laterals (Cases1, 2, 3 and 4) ........................................................ 124

Figure 6-7. Key performance indicators results based on the database for CO2-EGR\&S-CO2 Injection rate (Mscf/year)....................................................................................................................... 126

Figure 6-8.NN training, calibration and verification results (From left to right relatively) - CO2 Injection

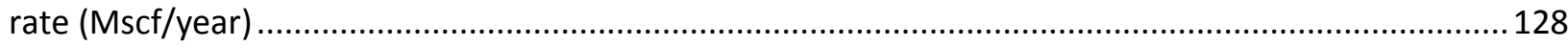

Figure 6-9.Comparison of CO2injection profile (Mscf/year) from simulator and CO2-EGR\&S Data-driven proxy model for some of the clusters (Cases1, 2, 3 and 4) ............................................................ 128

Figure 6-10.Comparison of CO2injection profile (Mscf/year) from simulator and CO2-EGR\&S Data-driven

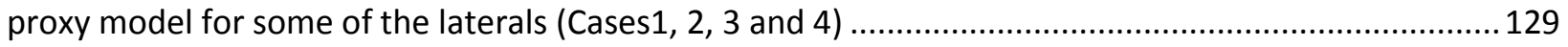

Figure 6-11.NN training, calibration and verification results (From left to right relatively) - CO2 BT time

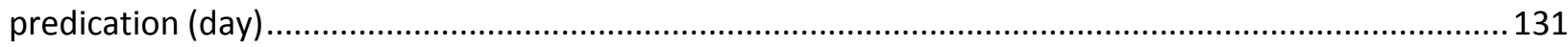

Figure 6-12 Key performance indicators results based on the database for CO2-EGR\&S-CO2 production rate (Mscf/year) ..................................................................................................................... 132

Figure 6-13.NN training, calibration and verification results (From left to right relatively) - $\mathrm{CO} 2$ Production predication (Mscf/year) ............................................................................................ 133 Figure 6-14.Comparison of $\mathrm{CO} 2$ production profile (Mscf/year) from simulator and CO2-EGR\&S Datadriven proxy model for some of the hydraulic fracture clusters (Cases1, 2, 3 and 4)........................... 134 Figure 6-15.Comparison of CO2 production profile (Mscf/year) from simulator and CO2-EGR\&S Datadriven proxy model for some of the laterals (Cases1, 2, 3 and 4) ...................................................... 134 
Figure 6-16.Comparison of $\mathrm{CH} 4$ production profile (Mscf/year) from simulator and CO2-EGR\&S Datadriven proxy model for some of the laterals -Blind Case. 135

Figure 6-17.Comparison of CO2 injection profile (Mscf/year) from simulator and CO2-EGR\&S Data-driven proxy model for some of the laterals -Blind Case. 136

Figure 6-18.Comparison of CO2 production profile (Mscf/year) from simulator and CO2-EGR\&S Datadriven proxy model for some of the laterals -Blind Case... 136

Figure 6-19. Schematic of new synthetic well for validation of the proxy models................................137

Figure 6-20. Comparison of $\mathrm{CH}_{4}, \mathrm{CO}_{2}$ production profile (Mscf/year) and $\mathrm{CO}_{2}$ Injection rate (Mscf/year) from simulator and CO2-EGR\&S Data-driven proxy model for some of the laterals -Blind Case............. 137 


\section{LIST OF TABLES}

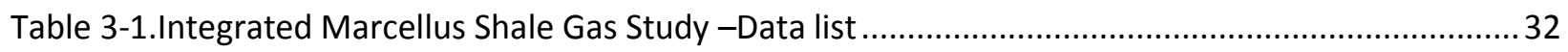

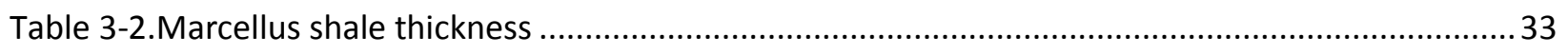

Table 3-3.Hydraulic fracturing hard data comparison-WVU pad and entire wells (77 wells) ................... 43

Table 3-4. History matching parameters and corresponding values for each well ..................................54

Table 4-1.Main input data for Data-driven shale proxy models development .......................................62

Table 4-2.List of optimum number of input for developing Data-driven proxy model (Daily basis)..........69

Table 4-3. List of optimum number of input for developing Data-driven proxy model (Monthly basis) ... 73

Table 4-4. List of optimum number of input for developing Data-driven proxy model (Annual basis) ..... 76

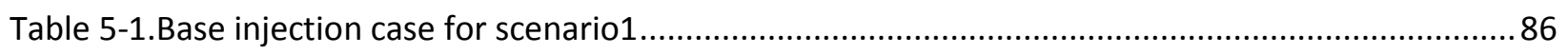

Table 5-2.Injection scenario 1-Case 1: Duration of injection and the amount of recovered $\mathrm{CH} 4$ and stored

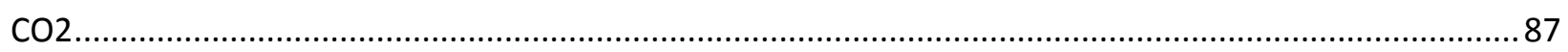

Table 5-3.Injection scenario 1-Case 2: Duration of injection and the amount of recovered $\mathrm{CH} 4$ and stored

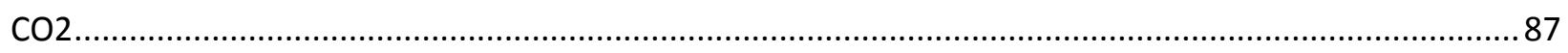

Table 5-4.Base injection case for scenario2 …................................................................................. 91

Table 5-5. Injection scenario 2-Case 1: Duration of injection and the amount of recovered $\mathrm{CH} 4$ and

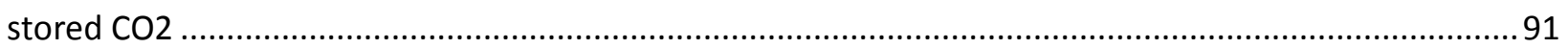

Table 5-6. Injection scenario 2-Case 2: Duration of injection and the amount of recovered $\mathrm{CH} 4$ and

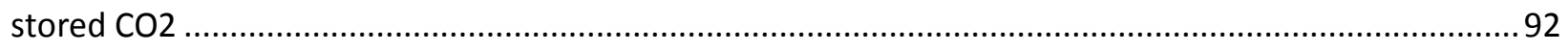

Table 5-7.Base injection case for scenario3 ................................................................................. 95

Table 5-8. Injection scenario 3-Case 1: Duration of injection and the amount of recovered $\mathrm{CH}_{4}$ and stored

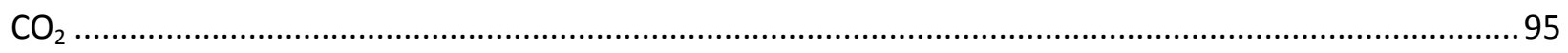

Table 5-9. Injection scenario 3-Case 2: Duration of injection and the amount of recovered $\mathrm{CH} 4$ and

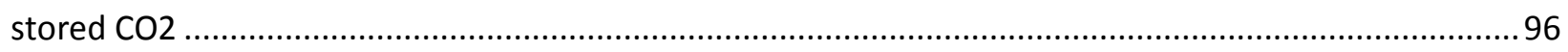

Table 5-10.Base injection case for scenario3 .................................................................................. 98

Table 5-11. Injection scenario 4: Duration of injection and the amount of recovered $\mathrm{CH}_{4}$ and stored $\mathrm{CO}_{2}$

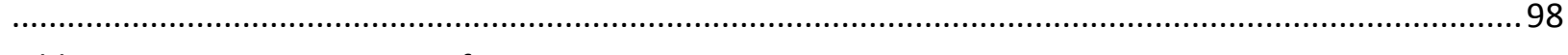

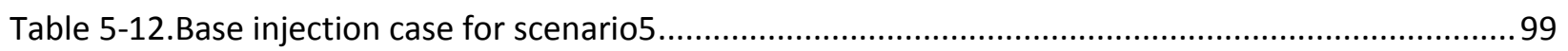

Table 5-13. Injection scenario 5-Case 1: Duration of injection and the amount of recovered $\mathrm{CH}_{4}$ and

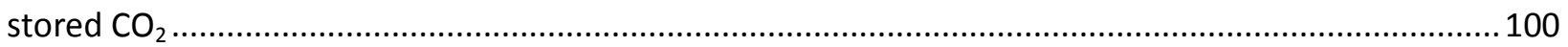

Table 5-14. Injection scenario 5-Case 2: Duration of injection and the amount of recovered $\mathrm{CH}_{4}$ and

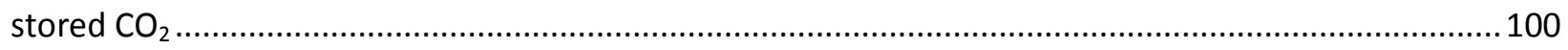

Table 5-15. Longmuir isotherms and selectivity ratio for sensitivity cases on VL-CH4 ......................... 105

Table 5-16. Longmuir isotherms and selectivity ratio for sensitivity cases on PL-CH4..........................108

Table 5-17. Longmuir isotherms and selectivity ratio for sensitivity cases on VL-CO2 .......................... 110

Table 5-18. Longmuir isotherms and selectivity ratio for sensitivity cases on PL-CO2 …...................... 112

Table 6-1.Main input data for $\mathrm{CO}_{2}$-EGR\&S Data-driven shale proxy models development .....................119

Table 6-2.List of optimum number of input for developing Data-driven CO2-EGR\&S proxy model........122

Table 6-3.List of optimum number of input for developing Data-driven CO2-EGR\&S proxy model........ 127

Table 6-4.List of optimum number of input for developing Data-driven CO2-EGR\&S proxy model........ 130

Table 6-5.List of optimum number of input for developing Data-driven CO2-EGR\&S proxy model........ 133 


\section{CHAPTER 1 INTRODUCTION}

\subsection{Background}

Shale gas production, as an unconventional source of natural gas, has had a long history in the United States, dating back to 1930 's. Shale gas has become an increasingly important source of natural gas in several regions of the world over the past decade. An analyst expects that shale gas will supply as much as half the natural gas production in North America by 2020 (Polczer, 2009). Figure 1-1 illustrates shale gas plays and related basins in the U.S. lower 48 states.

Shale gas is an unconventional gas reservoir contained in fine-grained, organic rich, sedimentary rocks, including shale, but composed of mud containing other minerals like quartz and calcite (Kennedy, 2012; U.S. DOE 2009; Warlick, 2010; U.S. EIA, 2011). As stated by Kennedy et al. (2012), a number of formations broadly referred to by the industry as shale, may contain very little shale lithology/mineralogy, but is shale by "grain size" only.

Shale gas formations are essentially lithified clays with organic matter present in varying amounts. The organic matter is believed to be an integral constituent of productive gas shale. Quantities of gas can be stored either as a dissolved phase in liquid hydrocarbons, or as an adsorbed phase on other materials within the shales of the kerogen, i.e., certain forms of illite. The phenomena of gas storage and flow in shale gas sediments are believed to be a combination of different controlling processes (Li, 2012). Gas is stored in three different ways in a shale reservoir: 1) Free Gas- in the rock matrix porosity and in the natural fractures, 2) Gas adsorbed (chemically bound) to the organic matter \&mineral surfaces within the natural fractures and/or absorbed (physically bound) to the organic matter and mineral surfaces within the matrix rock, 3) Dissolved-In the hydrocarbon liquids present in the bitumen (Kennedy, 2012).

According to Katsube (2000), gas flows through a network of pores with different diameters ranging from nanometers to micrometers .More specifically, the diameter of the pores in shale gas sediments ranges from a few nanometers to a few micrometers. In this very low permeability environment, gas (hydrocarbon) flow through the matrix is extremely limited and insufficient for commercial production. Various authors have estimated that a gas molecule will move no more than 10 to 50 feet per year through the shale matrix rock (Kennedy, 2012).

Unique characteristics of shale gas reservoirs causes generally less gas recovery (from $<5 \%$ to $20 \%$ ) relative to conventional gas reservoirs, although the naturally well-fractured shale like Antrim Shale may have a recovery factor as high as 50\%-60\%. 


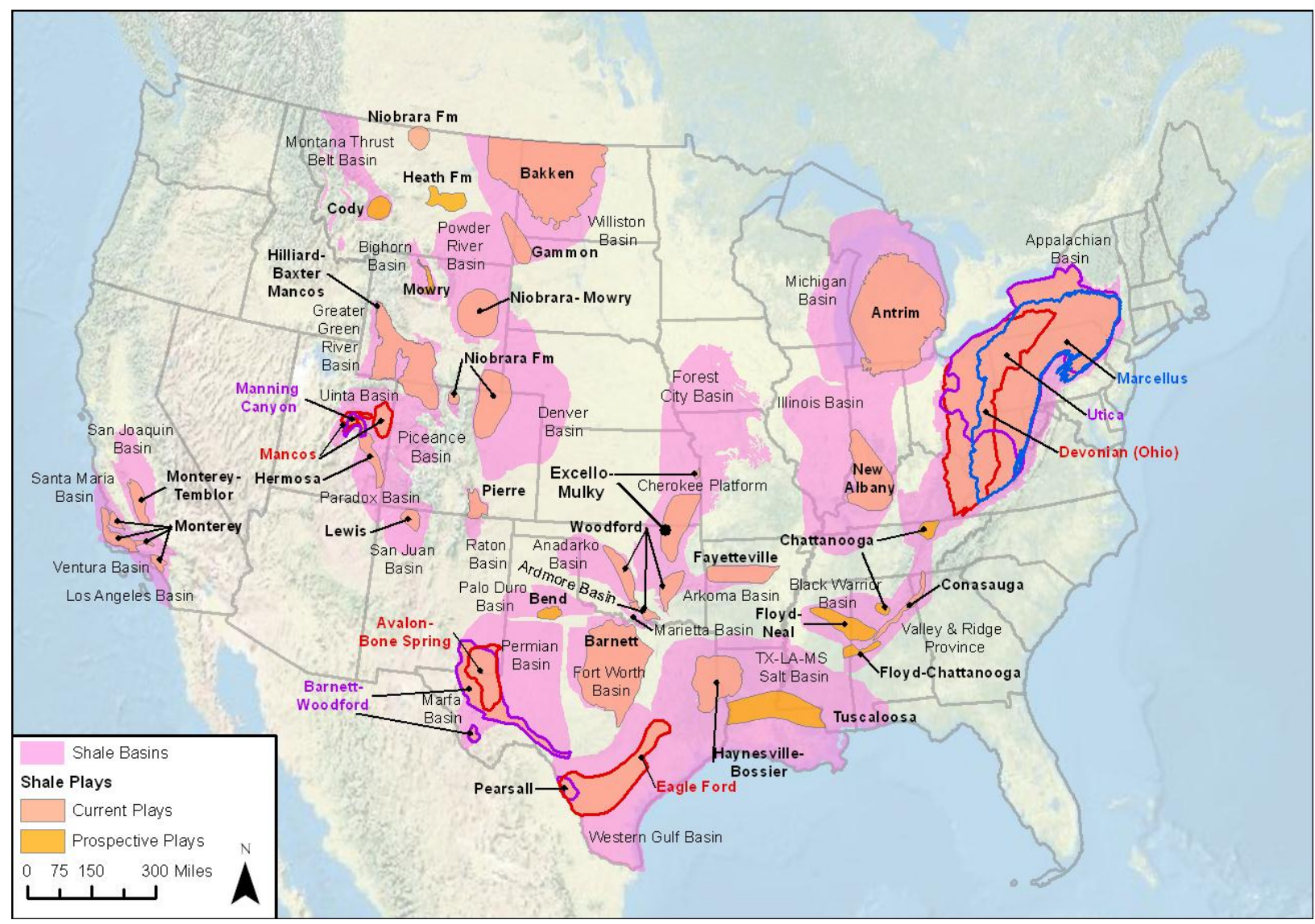

Figure 1-1. Map of shale gas plays and related basins in the U.S. lower 48 states. Sources: (U.S. Census Bureau, $2010 ;$ U.S. Energy Information Administration (EIA), 2011a, 2011b)

To increase the recovery factor, innovation in drilling and completion technology is essential in lowpermeability shale reservoirs. In recent years, improvements in the use of horizontal drilling combined with multi-stage/multi cluster hydraulic fracturing have resulted in some shale gas formations becoming some of the most attractive natural gas resources in the United States.

The more fractures in the shale around the wellbore, the faster the gas will be produced. Because of shale's extremely low permeability, the best fracture treatments are those that expose as much of the shale as possible to the pressure drop that allows the gas to flow. The natural formation pressure of a large gas shale reservoir will decline only slightly over decades of production. Any pressure drop on individual wells is likely the result of fractures closing up, rather than depletion of the reservoir. Therefore, the key to good shale gas production over time is having the proper distribution and placement of proppant to overcome the closure stress and keep the fractures open.

The Marcellus Shale has attracted a great deal of attention in the last few years as oil and gas operators explore for new sources of natural gas in locations close to the large markets in the northeastern United 
States. The Marcellus Formation is regionally extensive, covering large parts of Maryland, New York, Ohio, Pennsylvania, Virginia, West Virginia, and parts of Canada. It is also relatively thick (ranging from 40 to 900 feet in thickness), with low porosity $(\sim 0.09)$ and permeability $(5.9-19.6 \mu \mathrm{d})$ (U.S DOE, 2010).

In general, shale gas exploration and production has many requirements:

- Methods for handling multi scale heterogeneity and associated scaling relationships (sample-core, core-log, log-seismic).

- Knowledge of source-rock geochemistry, kerogen-rock interactions, and associate fluid-solid interactions.

- Understanding the drivers of reservoir/rock quality and the potential for accessing high quality reservoir sections via hydraulic fracturing.

- Understanding fracture containment and the drivers of fracture complexity. Including the associated problems of proppant transport, water trapping, and resulting low fracture conductivity.

Massive multi-cluster, multi-stage hydraulic fractures not only have proven to be essential for economic methane recovery from shale plays, but also in concurrence with competitive adsorption capacity of shale to carbon dioxide, may serve as an excellent sink for $\mathrm{CO}_{2}$ and have the added benefit of serving to enhance natural-gas production that could offset the cost of $\mathrm{CO}_{2}$ disposal.

The reservoir characteristics of Marcellus shale as it was explained, make it an excellent caprock formation for $\mathrm{CO}_{2}$ injected into deeper formations (Soeder, 1988), and by itself a possible geologic storage option, where the $\mathrm{CO}_{2}$ could be stored in an adsorbed state that is expected to be stable for geologically significant periods.

Figure 1-2 shows the extension of Marcellus shale play with producing wells and compare the $\mathrm{CO}_{2}$ generating industrial point sources (emitters) in southwestern Pennsylvania (SW PA) from two sources: the Environmental Protection Agency (EPA) and the Vulcan Project database (Gurney, 2009). The sinks are producing Marcellus shale wells when they produce their estimated ultimate accessible gas and at that point, the $\mathrm{CO}_{2}$ injection into the Marcellus shale is initiated. 

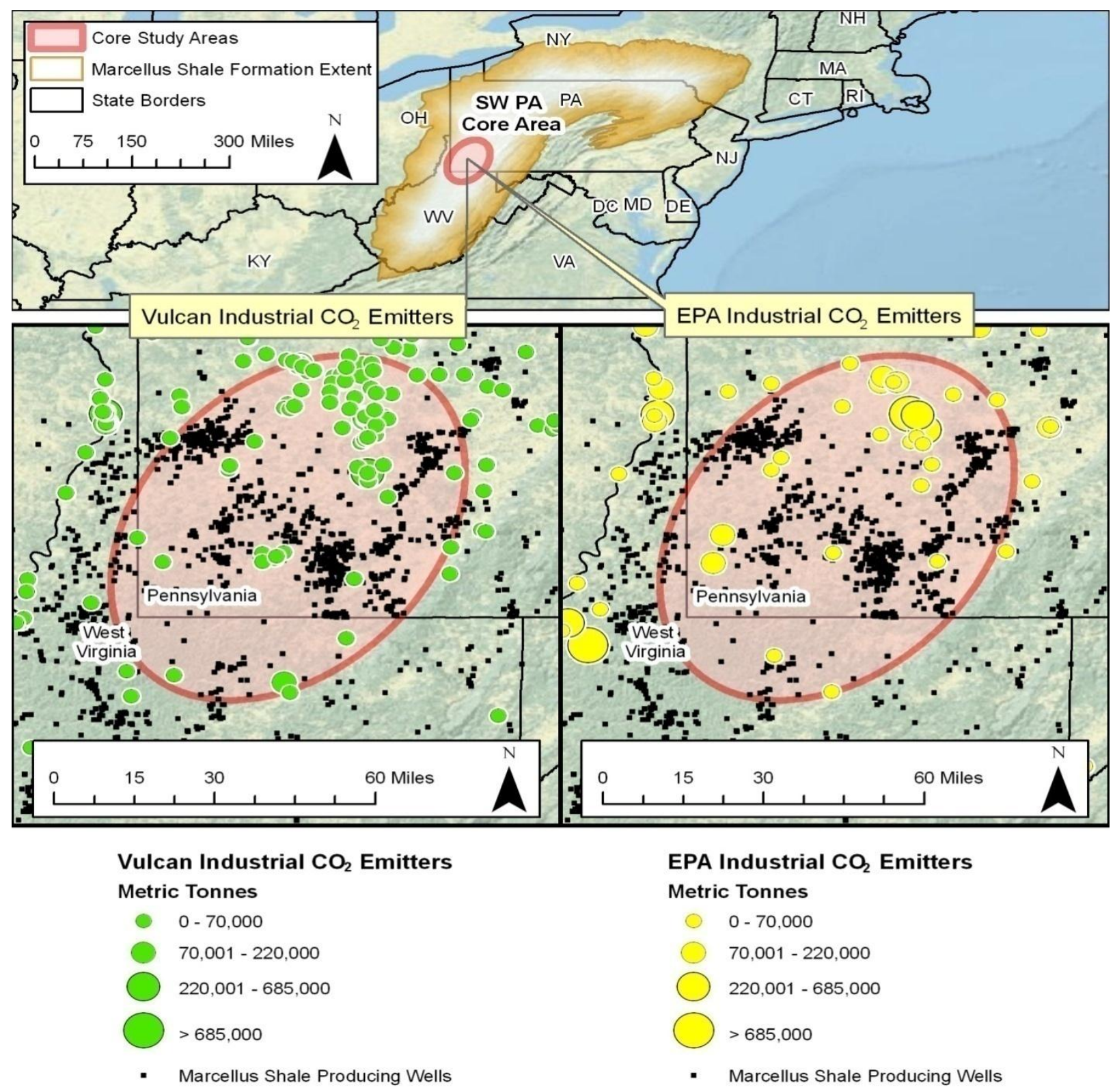

\section{EPA Industrial $\mathrm{CO}_{2}$ Emitters Metric Tonnes \\ $0-70,000$ \\ $70,001-220,000$ \\ $220,001-685,000$ \\ $>685,000$ \\ - Marcellus Shale Producing Wells}

Figure 1-2. Map of Marcellus shale play, Marcellus shale producing wells and $\mathrm{CO}_{2}$ emitters, Sources: (Gurney et al., 2009; NPS, 2009; U.S. Census Bureau, 2010; EIA, 2011b; EPA, 2012a; Zagorski et al., 2011)

\subsection{Problem Statement and Dissertation Contributions}

Latest advances in shale gas reservoir simulation and modeling have made it possible to optimize and enhance the production from organic rich shale gas reservoirs. Various attempts have been made to model fluid flow in shale gas systems. Models describing detailed physical processes can be built in a numerical setting where fractures can be discretely modeled and matrix blocks are assigned to transfer gas through diffusion and desorption into the fracture blocks (e.g. Lewis, 2004; Cipollaet al., 2009, Rubin, 2010; Wang, 2011; Mongalvy, 2011). 
The combination of flow through dual porosity, naturally fractured shale formation, and concentration gradient driven diffusion that is governed by Fick's law integrated with Langmuir isotherms that controls the desorption of methane into the natural fractures, has become the cornerstone of reservoir modeling in shale. The coupling of hydraulic fractures and natural fracture networks and their integration and interaction with the shale matrix remains the major challenge in reservoir simulation and modeling of shale formations.

Most commonly used numerical techniques for modeling production from shale wells are Explicit Hydraulic Fracture (EHF) and Stimulated Reservoir Volume (SRV). EHF modeling attempts to honor the impact of hydraulic fracturing at the cluster/stage level by incorporating hydraulic fracture characteristics in the simulation model. Model setup for the EHF technique is long and laborious and its implementation is computationally expensive, such that it becomes impractical to model and history match beyond a single pad. On the other hand, identifying the extent and conductivity of SRV is a challenging proposition. SRV technique is commonly used to simplify the modeling and the history matching process.

Apart from the complexity associated with developing a numerical simulation model, reservoir simulator is no longer used with a simple description of the complex shale gas reservoirs, but with multiple, equally probable realizations to allow risk assessment. Nevertheless, if we assume that we have full understanding of fluid flow mechanism in shale reservoirs, the perennial challenge in shale reservoir modeling is to strike a balance between explicit representation of reservoir complexity and long simulation run time for multiple realizations.

Exhaustive and comprehensive evaluation of the solution space for designing field development strategies as well as quantification of uncertainties associated with the static model are the type of analyses that require a large number of simulation runs in order to provide meaningful and usable results. When a numerical simulation model takes hours for a single run, performing such analyses become impractical and the engineers have to compromise by designing and running a much smaller number of runs in order to make decisions.

These problems in shale gas modeling caused engineers to rely heavily on the simplest, most accessible tool: such as using a reduced physics model (Wilson et al., 2012) or using the simplest production data analysis approach ( e.g. Decline curve analysis) by knowing the fact that tools of traditional production data analysis have not been sufficient identifying flow behavior/flow regimes in shale system. The 
problem will be even more noticeable, when trying to understand the physical process of $\mathrm{CO}_{2}$-Enhanced Gas Recovery and Storage $\left(\mathrm{CO}_{2}\right.$-EGR\&S) in shale formation.

The motives of this research is to couple numerical simulation and pattern recognition capabilities of artificial intelligence to model shale gas production at the hydraulic fracture cluster level and to evaluate $\mathrm{CO}_{2}$ injection process for the purpose of production enhancement and $\mathrm{CO}_{2}$ sequestration in the Marcellus shale gas reservoir.

To do so, an integrated workflow for shale gas simulation model development using a conventional numerical simulator, which incorporates the latest and best results from available core analysis; geological, petrophysical, and geophysical analyses; geomechanical analysis; and discrete (natural) fracture network, as well as multi-stage hydraulic fractures, is proposed. The history matching process is performed for a pad with six horizontal laterals and 169 clusters of hydraulic fractures.

To the date of writing this dissertation, there is no comprehensive reservoir simulation study in the literature to evaluate $\mathrm{CO}_{2}$-Driven Enhanced Gas Recovery and Storage $\left(\mathrm{CO}_{2}\right.$-EGR\&S) in shale. Therefore, the depleted history matched reservoir model is used to propose the best practices to enhance methane recovery, minimize $\mathrm{CO}_{2}$ production while maximizing the amount of stored $\mathrm{CO}_{2}$ in shale formations.

In order to facilitate the understanding of the massive potentials of the developed shale numerical reservoir simulation model for predicting production performance and EGR\&S that is needed to fulfill the modeling and simulation objectives, a next generation of proxy model, which is called Data-driven Shale Proxy model, based on pattern recognition capabilities of Artificial Intelligence is developed.

The developed Data-driven shale proxy model would be able to predict the production profile for different time resolutions at hydraulic fracture cluster level (Production phase) in a second. Besides, the predicted production rate at cluster level can be accounted as a synthetic PLT log for different time intervals. In addition, the developed Data-driven proxy model is capable of accurately reproducing the $\mathrm{CO}_{2}$ injection and $\mathrm{CH}_{4} / \mathrm{CO}_{2}$ production profiles and predicts $\mathrm{CO}_{2}$ breakthrough time $\left(\mathrm{CO}_{2}\right.$ Injection/Storage Phase) for each hydraulic fracture cluster and horizontal lateral and making it possible to perform detailed uncertainty and optimization studies of $\mathrm{CO}_{2}$-Enhanced Gas Recovery (EGR) and Storage process.

Coupled use of the deterministic reservoir model with Data-driven proxy model is expected to serve as a novel screening and optimization tool in evaluating the viability of residual gas recovery and $\mathrm{CO}_{2}$ storage 
in depleted (or near-depleted) shale gas formations.

\subsection{Dissertation Organization}

This dissertation has seven chapters that are organized in the following way:

\section{Chapter1- Introduction}

In this chapter, a brief overview of shale gas reservoirs in terms of their area of extension in the U.S. and their unique characteristics is presented and its potential as $\mathrm{CO}_{2}$ storage is highlighted. The challenges that are involved in the process of modeling and simulation of the production and also $\mathrm{CO}_{2}$-Enhanced Gas Recovery \& Storage $\left(\mathrm{CO}_{2}\right.$-EGR\&S) are addressed and the solution for overcoming these issues is presented as the contribution of this dissertation.

\section{Chapter 2- Literature Review}

This chapter presents a comprehensive literature review on shale gas numerical modeling and simulation techniques, $\mathrm{CO}_{2}$-EGR\&S and the application of proxy models in E\&P industry. Additionally, an overview of Artificial intelligence techniques especially neural networks, which are extremely powerful systems for pattern recognition and nonlinear multidimensional interpolation, is presented.

\section{Chapter 3-Shale Gas Numerical Simulation Development- Integrated Workflow}

Describes step-by-step procedure for developing an integrated workflow, which demonstrates a quantitative platform to model shale gas production, through capturing the essential characteristics of shale gas reservoirs. The steps are included: 1) Developing a base geological model, 2) Modeling the impact of the hydraulic fracture3) Incorporation of fracture (both natural and hydraulic) characteristics in the geological model 4) History matching the base model 5) Forecasting production for 100 years.

Chapter 4-Data-driven Model, A Next-Generation Shale Proxy at Hydraulic Fracture Cluster LevelProduction Phase

This chapter is focused on the development of Data-driven Shale Proxy Model based on the historymatched model at the hydraulic fracture cluster level using pattern recognition technology for different production time resolutions (Daily for the first two months, monthly for the first 5 years and annually for the 100 years). The developed proxies are further validated by completely blind simulation runs.

\section{Chapter 5-Technical Aspects of $\mathrm{CO}_{2}$-Driven Enhanced Gas Recovery and Storage in Depleted Shale}

This chapter gives a comprehensive analysis of the technical aspects of $\mathrm{CO}_{2}$-Enhanced Gas Recovery and storage in depleted shale gas reservoir. Different $\mathrm{CO}_{2}$ injection scenarios based on different well pattern (injector and producer pairs spacing), different reservoir characteristics, different sorption features and 
different operational constraint based on the history matched numerical simulation model are defined and the best practices to enhance methane recovery and to minimize $\mathrm{CO}_{2}$ production while maximizing the amount of stored $\mathrm{CO}_{2}$ in shale formation are proposed.

\section{Chapter 6-Data-driven Model, A Next-Generation Shale Proxy at Hydraulic Fracture Cluster Level-} Injection/Storage Phase

In this chapter, a detailed procedure for designing different simulation runs for making a spatio-temporal database, which is the core for the development of a Data-driven shale proxy model for $\mathrm{CO}_{2}$-driven enhanced gas recovery and storage, is presented. The resulting Data-driven proxy model is capable of accurately reproducing the injection and production profiles at each hydraulic fracture cluster and horizontal lateral and making it possible to perform detailed uncertainty and optimization studies of Enhanced Gas Recovery $\left(\mathrm{CO}_{2}\right.$-EGR) and Storage process.

\section{Chapter 7-Conclusions and Recommendations}

This chapter summarizes the research and its contributions, and gives future research recommendations. 


\section{CHAPTER 2 LITERATURE REVIEW}

\subsection{Introduction}

In this chapter, a comprehensive literature review on shale gas numerical modeling and simulation techniques, $\mathrm{CO}_{2}$-Enhanced Gas Recovery and Storage and the different type of proxy models and their application in E\&P industry is presented. In addition, an overview of Artificial intelligence techniques especially neural networks as a tool for pattern recognition and nonlinear multidimensional interpolations presented.

\subsection{Numerical Methods for Shale Gas Reservoir}

A vibrant and fast-growing literature exists related to various aspects of gas shales, including operational (e.g., drilling, completion, and production) and technological challenges. The latter mainly involves difficulties in formation evaluation/characterization, in modeling gas-matrix-fracture phenomena, and in developing reliable reservoir simulators .In times, these studies directly point to difficulties in accurately predict the ultimate gas recovery and to explain high variability in gas well productivity, which are common to nearly all shale gas reservoirs (Fathiet al., 2009).

There is currently considerable focus on the reserves estimation and also on optimizing the production from shale formation through advanced completion technology and numerical reservoir simulation.

The current state of reservoir modeling technology for shale uses the lessons-learned from modeling naturally fractured carbonate reservoirs and those from Coalbed Methane reservoirs. Therefore, applying conventional knowledge and methodologies to unconventional reservoirs could be a humbling experience.

Dual continuum models are the conventional method for simulating fractured systems and are widely used in the industry (Figure 2-1).Barenblatt et al. (1960) and Warren-Root (1963) first developed mathematical models describing the fluid flow in dual-porosity media. These models assume single-phase fluid in pseudo-steady-state, transfer from matrix to fracture. Kazemi (1969), Rossen (1977), and deSwaan (1976) and Saidi (1983), extended the Warren and Root approach to multiphase flow and developed dual porosity simulators, which considered un-steady-state or transient fluid transfer between matrix and fracture. These models represented naturally fractured media as a set of uniform matrix blocks and fractures. 


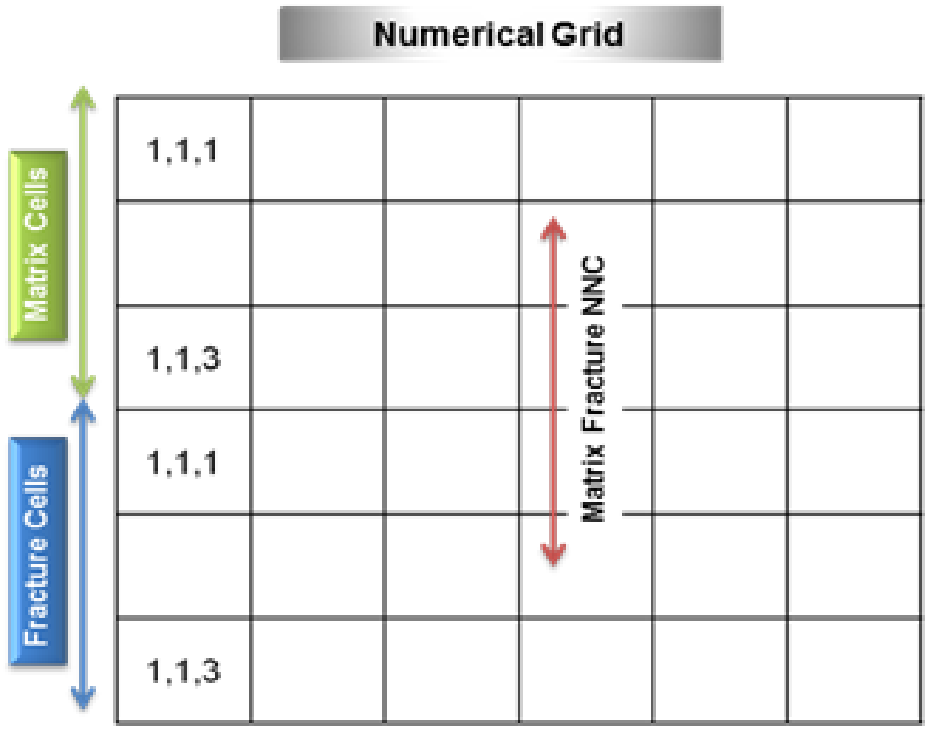

Conceptual Model

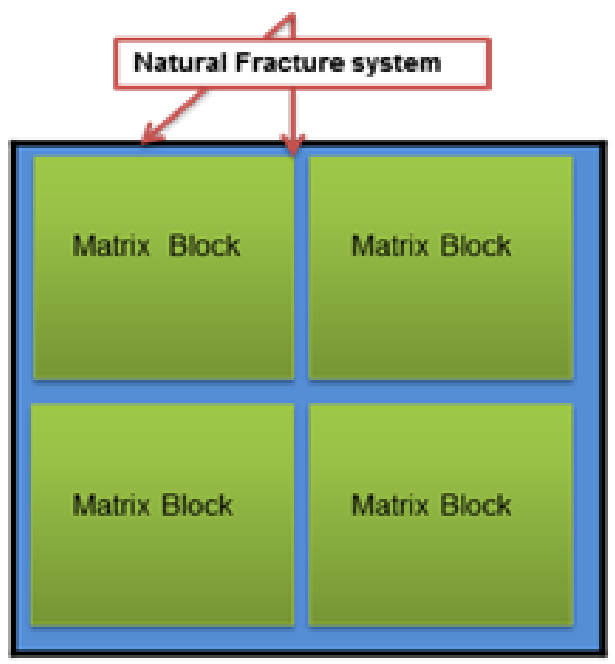

Figure 2-1. Dual porosity numerical and conceptual model (Kalantari-Dahaghi 2011)

Later, Blaskovich et al. (1983), Hill and Thomas (1985), and Dean and Lo (1986) developed dual permeability models, which allow for matrix to- matrix flow, but these reservoirs are modeled as a continuum.

In addition, a dual permeability grid is used to allow simultaneous matrix-to-matrix and fracture-tofracture flow. This method can model transient gas production from hydraulic fractures of the horizontal wells in shale gas reservoirs (Rubin, 2010; Cipolla et al., 2010).

Dual-Mechanism Approach (Darcy flow and Fickian diffusion occur parallel in matrix) was introduced to characterize the gas flow in coal or shale formations through the dynamic gas slippage factor (Ertekin, et al., 1986; Clarkson, et al., 2010).

Javadpour (2009) first derived the concept of apparent permeability considering the Knudsen diffusion, slippage flow and advection flow, and it was further applied to pore-scale modeling for shale gas (Shabro et al.,2011 and Shabro et al.,2012). Based on a unified Hagen-Poiseuille-type formula, Beskoket al. (1999), Civan et al. (2010) and Ziaraniet al.(2012) proposed a method to calculate apparent permeability through the flow condition function (a function of Knudsen number) and the intrinsic permeability of porous media.

Yen et al. (2013) believed that the validity of this latter model for different flow regimes still requires further confirmation. However, even with this greater detail, these models may not be sufficient due to the 


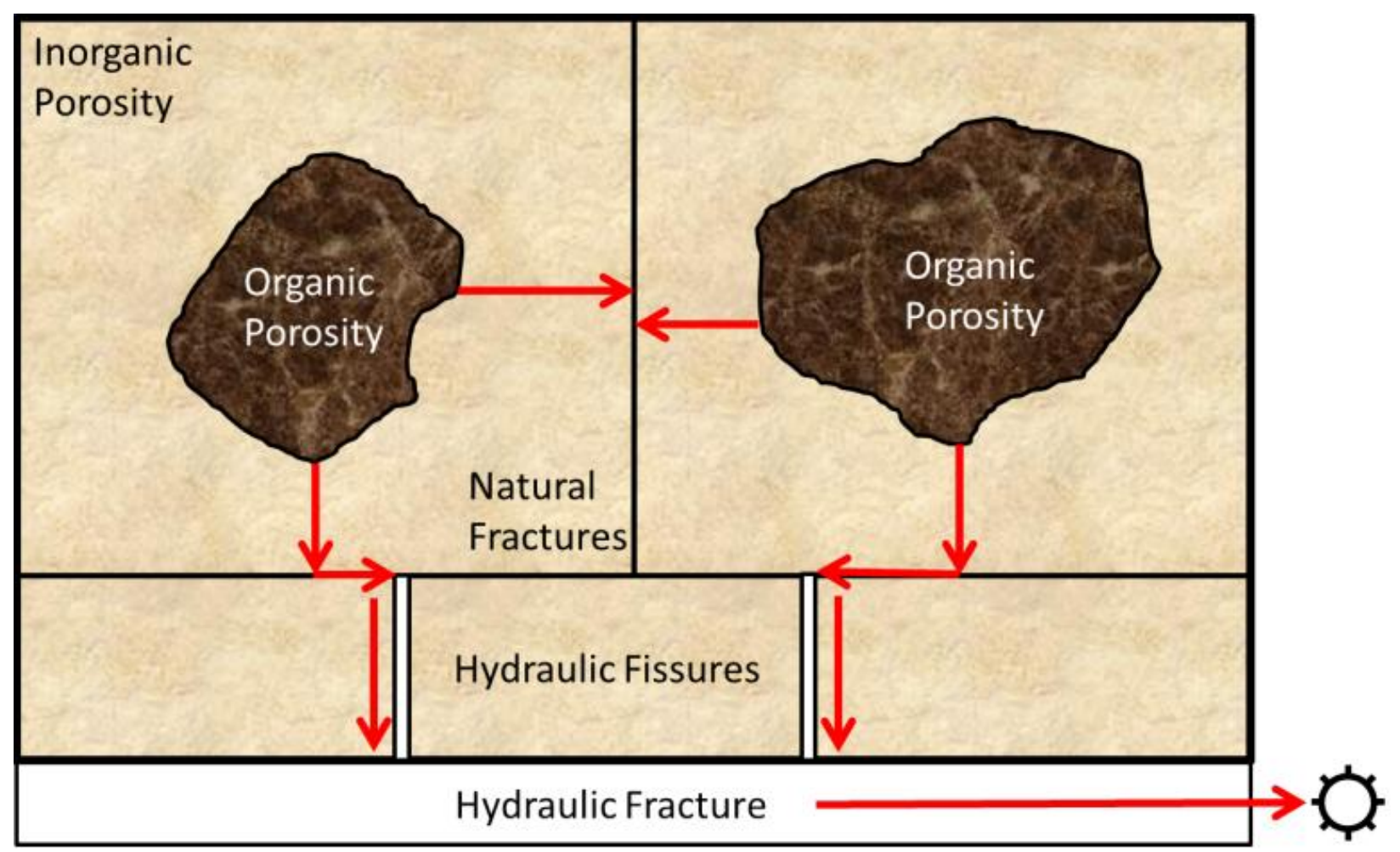

Figure 2-2. Realization of shale-gas reservoir sample with components in series. The arrows indicate direction of gas flow (Hudson 2011)

use of an undivided shale matrix. Even with a proper connectivity between different pore systems, there is still a little understanding of this aspect (Andrade et al., 2011). On the other hand, Hudson et al.(2011, 2012) did categorize the shale reservoir into organic porosity, inorganic porosity, natural fractures and hydraulic fractures (Figure 2-2), and explored several different tank models for connections between each pore system.

Unfortunately, the models to characterize the distribution of each continuum and the connections between those continua appear too regular to be realistic for shale reservoirs. Clearly, there remains a strong requirement to accurately model production from unconventional reservoirs based on detailed physics of the process and the interaction between two different porosities such that modeling uncertainty is reduced (Yen et al.2013).

On the other hand, hydraulic fractures are the main reason for economic production from shale and given the complex nature of hydraulic fracture growth and their interaction with the rock fabric, they becomes one of the most important aspects of modeling storage and flow in shale formations. However, the presence of the massive multi-cluster, multi-stage hydraulic fractures makes the reservoir modeling of shale formation even more complicated. 
There are several ways of modeling hydraulic fractures characteristics and their interaction with natural fractures using analytical and semi-analytical techniques before going to reservoir simulation. MFrac ${ }^{\mathrm{TM}}$ and FracPro ${ }^{\mathrm{TM}}$ are two examples of software packages that can be used to calculate the characteristics of an idealized penny-shaped hydraulic fracture by using the hydraulic fracture job characteristics (hard data) such as fluid and proppant amount and rate and pressure of injection, along with some reservoir characteristics and stresses.

In order to implement the calculated hydraulic fracture characteristics to numerical simulation, a proper gridding technique is required. All the existing approaches can be ultimately divided into two distinct groups. The first is the Explicit Hydraulic Fracture (EHF) modeling method, and the second is known as Stimulated Reservoir Volume (SRV).

Explicit Hydraulic Fracture (EHF) modeling is the most complex (as well as the most robust) approach for modeling the impact of hydraulic fracturing during numerical simulation of production from shale. EHF modeling attempts to honor the impact of hydraulic fracturing at the cluster/stage level by incorporating hydraulic fracture characteristics in the simulation model.

Model setup for the EHF technique is long and laborious and its implementation is computationally expensive, such that it becomes difficult or in some cases impractical to model beyond a single pad. By looking at the numerical reservoir simulation modeling efforts concentrated on shale assets, it can be noticed that almost all of the published studies are concentrated on analyzing production from single wells (Bazan et al., 2010; Chaudhri, 2012; Meyer et al., 2010; Cipolla et al., 2010a, 2010b; Samandarli et al., 2011). This confirms the complexity and computational prohibitively of using this technique specially in the case of performing full-field simulation.

The second technique for modeling production from shale wells is known as Stimulated Reservoir Volume (SRV) modeling technique. Stimulated Reservoir Volume (SRV) modeling technique is a different and much simpler way of handling the impact of massive multi-cluster, multi-stage hydraulic fractures in numerical reservoir simulation and modeling.

Using SRV instead of EHF can expedite the modeling process by orders of magnitude. This is due to the fact that instead of exactly modeling every individual hydraulic fracture, in this method the modeler assumes a three dimensional volume around the wellbore with enhanced permeability as the result of the hydraulic fractures. By modifying the permeability and the dimensions of the Stimulated Reservoir Volume (SRV), the modeler can now match the production behavior of a given well in record time. 
Nevertheless, there are two main issues with SRV concepts: 1) How one would calculate, or more accurately, estimate, the size of the Stimulated Reservoir Volume? 2) Is SRV a continuous medium or it has discrete characteristics for each hydraulic fracture and whether or not these discrete volumes are connected to one another? Furthermore, how are the aspect ratios (ratio of height, to width and to length) of the Stimulated Reservoir Volume determined? (Mohaghegh 2013)

As discussed by Suliman et al. 2013, during the last few years, the concept of Stimulated Reservoir Volume (SRV) has been linked to microseismic. Numerous published papers addressed the geometry and shape of the SRV created around horizontal wellbores in shale reservoirs using microseismic monitoring techniques with simple diagnostic plots (Daniels et al.2007, Fisher et al.2002, Maxwell et al.2002). Most of these efforts had not been calibrated quantitatively by mathematical correlations to production data and depletion efficiency.

Mohaghegh (2013) believes that the evidence that supports the estimation of the size of the Stimulated Reservoir is countered equally by evidence that negates it. Furthermore, it has been shown that misinterpreting the size of the Stimulated Reservoir Volume can result in large discrepancies in forecasting the potentials of a given well.

The sensitivity of production from shale wells to the size and the conductivity assigned to the Stimulated Reservoir Volume explains the uncertainties associated with the forecasts that are made using this technique.

Although there have been attempts to address the dynamic nature of the SRV (Suliman et al.2013) by incorporating Stress Dependent Permeability (opening and closure of the fractures as a function of time and production), the entire concept remains in the realm of creative adaptation of existing tools and techniques to solve a new problem. In the opinion of the author, while SRV serves the purposes of modeling and history matching the observed production from a well, its contribution to forecasting the production (looking forward) is questionable at best.

A recent study by Ciezobka (2012) demonstrated that the calculated SRV by itself is not always reliable and other factors are needed to be considered in order to predict production. In that study, the estimated SRV cloud was compared with the associated gas production contribution from each stage by running PLT on a pad with six horizontal laterals in the Marcellus shale.

The interesting point was that the production rates from two laterals were almost triple the production rates of other wells, which had higher calculated SRV, almost double in magnitude. 

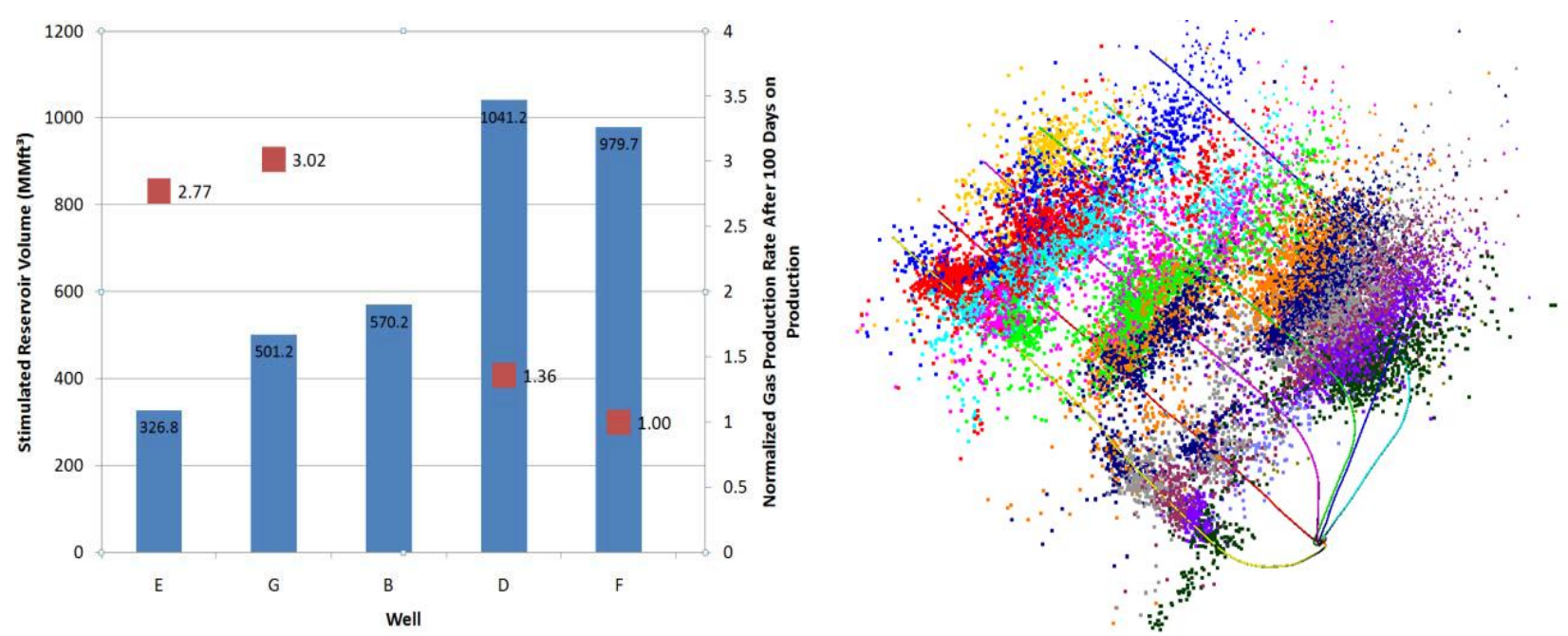

Figure 2-3. Comparison of SRV and normalized gas production from each stage and Map View of Microseismic Events (left to right)

Because of the uncertainty and complexity associated with the reservoir simulation model, analytical workflows like Rate Transient Analysis (RTA) and data centric workflows like Decline Curve Analysis (DCA) are widely used (Zhou et al. 2013).

Lee et al. (2010) believe that the time and effort required to history match individual wells, when production forecasts for hundreds of wells must be provided in a short period of time. Thus we must continue model development, while we depend on less rigorous approached (such as empirical decline curve models) for much of our production forecast and reserve estimation.

Decline Curve Analysis (DCA) is a well-known, easy to use, and popular technique in the petroleum industry. When applied to shale wells, DCA has many shortcomings. Several authors (Mattar et al. 2008; Boulis et al.2009; Johnson et al. 2009; Cheng et al.2010; Can et al. 2012; Ikewun et al. 2012) have come up with interesting techniques to overcome some of the well-known shortcomings of DCA, but nevertheless, many facts remains that make the use of Decline Curve Analysis suboptimal.

One of the major criticisms of Decline Curve Analysis is its lack of sensitivity to major physical phenomena in shale wells that has to do with the fluid flow, the hydraulic fracture, and the reservoir characteristics. In cases like Marcellus and Utica shale reservoirs, where short periods of production are available, the use of Decline Curve Analysis becomes increasingly problematic because at different production time intervals, well will be in different flow regimes that cannot be captured by DCA .

There are many studies in the literature about the Rate Transient Analysis (RTA) (Bello and Wattenbarger, 2008; Al-Ahmadiet al., 2010; Anderson et al., 2010; Nobakhtet al., 2010; Ilk et al., 2011; 
Nobakht and Clarkson, 2012; Nobakht and Mattar, 2012;) that approximates the essence of reservoir simulation and modeling using a series of analytical approaches. RTA is based on the transient solution of the pressure diffusion equation that governs fluid flow in porous media. RTA's ease of use and consistency of results are among its strongest points. On the other hand using RTA with no further information about fracture geometry could be very misleading (Clarkson 2011).

\section{3 $\mathrm{CO}_{2}$-Enhanced Gas Recovery and Storage in Shale Reservoir}

Increased emissions of carbon dioxide $\left(\mathrm{CO}_{2}\right)$, especially from the combustion of fossil fuels, are being linked to global climate change and are of considerable concern. These concerns are driving initiatives to develop carbon management technologies, including geologic sequestration of $\mathrm{CO}_{2}$. At present, several geological $\mathrm{CO}_{2}$ sequestration technologies, such as $\mathrm{CO}_{2}$ injection into saline aquifer, $\mathrm{CO}_{2}-\mathrm{EOR}, \mathrm{CO}_{2^{-}}$ ECBM, and so forth, have been studied to minimize the $\mathrm{CO}_{2}$ release into the atmosphere, and these projects have been operating all over the world(Stevens et al.1998, Ennis-King et al.2002,Orr 2004, Sinayuçet al.2008, Petrusaket al.2009, Liner 2009).

In general, physical properties of $\mathrm{CO}_{2}$, such as density and viscosity, are highly depend on the reservoir condition of the underground storage, which controls the mobility and occupied volume by $\mathrm{CO}_{2}$ in the reservoir. According to Essendelf et al. (2006) study, a large volume changes are associated with $\mathrm{CO}_{2}$ phase changes (under reservoir condition), as a result, it might be desirable to store $\mathrm{CO}_{2}$ under physical conditions that are not close to the phase boundary.

The typical $\mathrm{CO}_{2}$ phase diagram is shown in Figure 2-4. According to this figure, $\mathrm{CO}_{2}$ exists in the supercritical (dense) phase condition at above its critical pressure of $7.38 \mathrm{MPa}$ (1071 psi) and critical temperature of $31.1^{\circ} \mathrm{C}\left(88^{\circ} \mathrm{F}\right)$.

At supercritical conditions, $\mathrm{CO}_{2}$ has a density similar to a liquid and viscosity and diffusivity comparable to a gas (Gupta 2006). It is desirable to store $\mathrm{CO}_{2}$ as a supercritical fluid or a liquid because of higher phase density that will occupy a much smaller volume in the subsurface at supercritical pressure and temperature than it would be at surface pressure and temperature (Essendelf et al., 2006).

For example, 1 short ton of $\mathrm{CO}_{2}$ gas at surface temperature and pressure occupies a volume of 18,000 $\mathrm{ft}^{3}$, whereas supercritical $\mathrm{CO}_{2}$ at a depth of $2,600 \mathrm{ft}$. below the surface occupies a volume of $50 \mathrm{ft}^{3}$ (Wickstrom and others, 2005). 


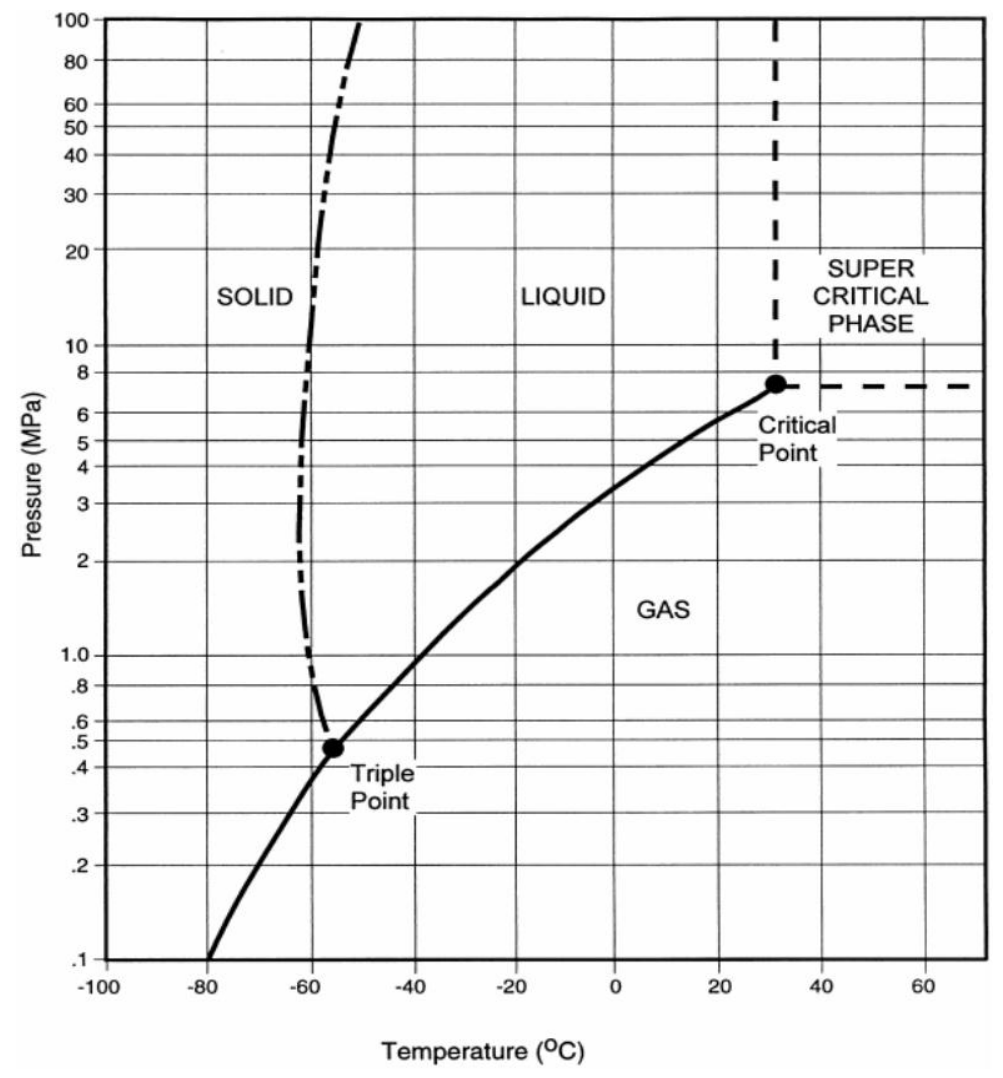

Figure 2-4. $\mathrm{CO}_{2}$ Phase Diagram (Essendelfet al., 2006)

One option for sequestration may be black shales, organic-rich rocks that are both the source and trap for natural gas (primarily methane). In shale gas reservoirs, natural gas occurs as free gas in the intergranular and fracture porosity and is adsorbed on clay and kerogen surfaces, very similar to the way methane is stored within coal beds.

It has been demonstrated in gassy coals that on average; $\mathrm{CO}_{2}$ is preferentially adsorbed, displacing methane at a ratio of two for one or more. Black shale reservoirs may react similarly and desorb methane in the presence of adsorbing $\mathrm{CO}_{2}$. If this is the case, black shales may serve as an excellent sink for $\mathrm{CO}_{2}$ and have the added benefit of serving to enhance natural-gas production (Nuttall et al., 2005, Schepers et al., 2009).

The continuing development of the Marcellus Shale, one of the largest natural gas plays in the United States, has the potential to and positively impact the future of Carbon Capture \& Sequestration (CCS) in the Appalachian Basin. Continuous, low-permeability, fractured, organic-rich gas shale units are widespread and are possible geologic storage targets. The Marcellus could act as a sealing (cap rock) formation for injection of $\mathrm{CO}_{2}$ into limestone and sandstone formations below the Marcellus, and (2) the Marcellus itself could act as a storage reservoir for captured $\mathrm{CO}_{2}$. In this scenario, Marcellus could 
provide favorable $\mathrm{CO}_{2}$ adsorption as well as available pore space from formation stimulation/fracturing and additional layers above the Marcellus would act as seals (U.S. DOE 2010).

There are few active sites involved in $\mathrm{CO}_{2}$ sequestration and enhanced gas recovery in CBM reservoirs, but according to Seto (2009), there is no field operation of gas injection in shale reservoirs up to date and there is no detailed numerical modeling and simulation study as well. At the time of writing this dissertation, only one pilot test has been done in Kentucky to examine the potential of $\mathrm{CO}_{2}$ storage and EGR in Devonian shale of Eastern Kentucky (Nuttall et al., 2005,Schepers et al., 2009).A very simple simulation model was built by Schepers et al. (2009) with only one well to investigate $\mathrm{CO}_{2}$ plum extension.

Kang et al. (2011) presented an experimental study on the ability of organic rich shale core samples (from the Fort Worth basin) to store carbon dioxide. Although their study was important for understanding of gas storage and transport in very specific organic rich shale, but the result may not necessarily be applicable to the other shale formations in US due to their difference in terms of organic content, pore structure of organic materials etc.

Therefore, there is a great need for performing comprehensive simulation studies to better understand $\mathrm{CO}_{2}$ injection process in shale gas reservoirs by considering different reservoir characteristics, sorption features, operational constraint and well patterns configuration.

The potential storage resource has not been computed for the Marcellus Shale, but given the greater depths and higher organic content, it could be extremely large.

Technical and economic challenges to $\mathrm{CO}_{2}$ geologic storage and enhanced gas recovery from shale gas reservoirs include (1) potential reduction of the permeability of already low-permeability (nano-darcy) shale due to differential swelling, similar to that of coal beds, and (2) the potential negative impact on long-term natural gas production, due to $\mathrm{CO}_{2}$ contamination of producing methane (U.S. DOE 2010).

Based on data for the adsorption of $\mathrm{CO}_{2}$ onto organic shales of $14 \mathrm{scf} /$ ton shale to $136 \mathrm{scf} / \mathrm{ton}$ shale at $400 \mathrm{psi}$ and the following Marcellus Formation characteristics,

Density $=159 \mathrm{lb} / \mathrm{ft}^{3}$

Area $=95,000 \mathrm{mi} 2$

Average Thickness $=100 \mathrm{ft}$. 
$\mathrm{CO}_{2}$ Density $_{\mathrm{Gas}}=5.8 \mathrm{e}-5 \mathrm{scf} / \mathrm{ton}$

the procedure by Nuttall et al. (2005) can be used to estimate the $\mathrm{CO}_{2}$ storage potential across the entire Marcellus Formation, which, as a whole, has the potential to store from 17 to 166 billion tons of $\mathrm{CO}_{2}$ (U.S. DOE-NETL 2010).

As with any developed or under developing oil/gas field, existing wellbores (pads with multiple horizontal laterals) will be a concern that needs to be addressed. The placement, type, and number of well penetrations can have implications for future geologic storage opportunities in regions overlying the Marcellus. As stated by U.S. DOE (2010), while Marcellus shale horizontal wells provide the opportunity to acquire natural fuel resources, they might create potential leakage conduits for $\mathrm{CO}_{2}$ either injected into deeper formations (like the Oriskany Sandstone) or into the vacant fractured spaces in the Marcellus Formation, possibly creating avenues for $\mathrm{CO}_{2}$ to migrate to shallower formations. However, this potential concern could be modeled, identified, and mitigated with risk assessment techniques.

In unconventional reservoir simulators, the diffusive flow between the matrix and the fracture (during production and injection) is given by:

$$
F_{i}=D I F F M F * D_{c, i} * S_{g} * R F_{i}\left(m_{i}-\rho_{s h} L_{i}\right)
$$

Where:

$m_{i}=$ Molar density in the shale matrix.

DIFFMF = Matrix fracture diffusivity.

$P_{s h}=$ Rock density (shale density)

$D_{c, i}=$ Diffusion coefficient (shale) component $i$

$R F_{i}=$ Readsorption factor component $i$,

$S_{g}=$ Gas saturation, for desorption a value of unity is used.

$\rho_{s h} L_{i}=$ equilibrium adsorbed molar density

$\rho_{\text {sh }}=$ shale density

The matrix fracture diffusivity is given by:

$\mathrm{DIFFMF}=\operatorname{DIFFMMF} * \mathrm{Vol} * \sigma$

Where:

DIFFMMF $=$ multiplying factor $($ default $=1.0)$ 
$V o l=$ cell shale volume and $\sigma$ is the factor to account for the matrix-fracture interface area per unit volume.

Often the sorption times are a quantity that is easier to obtain than the diffusion coefficients. For desorption we write the flow as:

$$
\mathrm{F}_{\mathrm{i}}=\frac{\mathrm{Vol}}{\tau_{\mathrm{i}}} *\left(\mathrm{~m}_{\mathrm{i}}-\rho_{\mathrm{c}} \mathrm{L}_{\mathrm{i}}\right)
$$

Where:

$$
\tau_{\mathrm{i}}=\frac{1}{\mathrm{D}_{\mathrm{c}, \mathrm{i}} * \mathrm{DIFFMMF} * \sigma}
$$

is called the sorption time. The parameter controls the time lag before the released gas enters the shale fracture system. The sorption times are given by the diffusion coefficients and the matrix-fracture interface area together with the multiplying factor DIFFMMF. If the sorption times are known a value of unity can be assigned to and DIFFMMF. The diffusion coefficients can then be assigned to the reciprocal of the sorption times.

The amount of gas contained or adsorbed in the shale at equilibrium conditions can be calculated using the Langmuir isotherm equation. The general Langmuir isotherm equation for gas is the following:

$V(P)=\frac{V_{L} * P}{P_{L}+P}$

The maximum volume of gas adsorbed at infinite pressure is called Langmuir Volume. Langmuir pressure is determined by taking the pressure at about half of the Langmuir volume. Having measured values for gas content, Langmuir volume and Langmuir pressure, then the critical pressure can be calculated as indicated in the following equation 2. The critical pressure at which Methane starts flowing is proportional to the gas production capability. Higher pressure values are related to larger amount of gas produced.

$$
\mathrm{P}_{\mathrm{C}}=\frac{\mathrm{P}_{\mathrm{L}} * \mathrm{G}_{\mathrm{C}}}{\mathrm{V}_{\mathrm{L}}-\mathrm{G}_{\mathrm{C}}}
$$

The Extended Langmuir isotherm is used to describe the shale sorption for the different components. The adsorption capacity is a function of the pressure and the free gas phase composition. For each component, 
two parameters need to be input, the Langmuir volume constant and the Langmuir pressure constant. These parameters are typically determined from experiments. The multi-component adsorption capacity is calculated by:

$$
\begin{aligned}
& \mathrm{L}\left(\mathrm{p}, \mathrm{y}_{1}, \mathrm{y}_{2}, \ldots\right)_{i} \\
& =\theta \frac{P_{s}}{\mathrm{RT}_{S}}\left\lfloor\mathrm{~V}_{i} \frac{\mathrm{y}_{i} \frac{p}{P_{i}}}{1+\sum_{i=1}^{n c} \mathrm{y}_{j} \frac{p}{P_{j}}}\right\rfloor \ldots
\end{aligned}
$$

Where:

$\theta=$ Scaling factor

$P s=$ Pressure at standard conditions

$R=$ Universal gas constant

$T_{S}=$ Temperature at standard conditions

$V i=$ Langmuir volume constant comp. $i$

$P i=$ Langmuir pressure constant comp. $i$

$y i=$ Hydro carbon mole fraction in gas phase comp. $i$

$p=$ Pressure

\subsection{Proxy Models and Uncertainty Analysis}

Proxies are fast approximates to full-scale engineering simulation. They have been used extensively for more than a decade in many industries including the aerospace industry and weather forecasting. They have become popular in the E\&P industry over the past several years (Goodwin et al., 2012). They are mathematically/statistically defined functions that replicate simulation model output for selected input parameters and provide fast-approximated solutions that substitute large numerical simulation models and are used to fulfill many different purposes.

As stated by Goodwin et al. (2012), in the reservoir engineering world, proxies have been found useful for accelerating the history-matching process, and they can cope with the large number of samples required for Monte Carlo (MC) simulations. Moreover, they are used to assist in the field development planning, uncertainty analysis, optimization of operational design, and history matching. Most common proxy models are either reduced models (increase run-time speed by grossly approximating the problem) or response surfaces (grossly approximating the solution space). 
Current state of building proxy models, when it comes to representing numerical reservoir simulation models, leaves much to be desired. Nevertheless, they are routinely developed and used in order to generate fast solutions to changes in the input space.

Given the fact that computational power has increased vastly in recent years, an argument can be made about the role and even necessity of developing proxy models. However, Williams et al. (2004, 2006) observe that gains in computational power "are generally used to increase the complexity of the models rather than to reduce model run time." Therefore, still there is a need in the development of effective and robust proxy models.

In order to build an effective proxy model, an appropriate Experimental design method is needed to build a comprehensive and informative data set. Moreover, Experimental design is the most widely used methods in the industry for uncertainty analysis and sensitivity analysis given the uncertain parameters. The basic idea behind this methodology is to vary multiple parameters at the same time so that maximum inference can be attained with minimum cost.

Once the appropriate design is established and the corresponding experiments (simulations) are performed, the results can be investigated by fitting them to a response surface that can be used as a proxy to reservoir simulation to quantify the uncertainties (Friedmann et al. 2003, Yeten et al. 2005, Xie et al. 2013). The experimental design method is more efficient than the direct Monte Carlo method.

The most prominent and commonly used approaches for developing a proxy model that can be used in reservoir simulation and modeling are as follows:

RSM-Polynomial regression models are especially suitable in cases, where the problem is known to be governed predominantly by low-order effects (linear or quadratic) and the number of input variables is limited ( $\mathrm{n} \leq 10)$. When defining the setup of RSMs, the only free choice is in the regressors. On the one hand, this limited number of options makes RSM easy to handle, but on the other hand, it restricts the range of possible applications (Myers et al 2002).

MLS-The moving-least-squares approach makes use of regression techniques to define a global model based on a locally weighted polynomial approximation, which typically does not interpolate the observations. Accordingly, MLS models are favorable whenever RSMs cannot be established to be globally valid, but the function to be approximated is smooth enough such that in the proximity of the prediction point a polynomial relationship can be accepted (Jurecka 2007). 
Kriging- This type of proxy model is flexible due to the large variety of feasible correlation formulations but from a computational point of view, kriging models are less favorable. For the fitting process, an ndimensional optimization is necessary to find the correlation parameters via maximum likelihood estimation (Jakumeit et al. 2005).

RBF- Radial basis function approximations are comparable to kriging models. In this technique, the flexibility to adapt to many different applications is due to the variety of radial basis functions. The accuracy of the obtained approximation is affected by the opted radial basis function and its free parameter. Similar to the problem of specifying the weighting functions for MLS approximations, there is no general rule how to find the best radial basis function for a problem (Krishnamurthy 2003).

The probabilistic collocation method (PCM) is another efficient stochastic approach. It has been applied to uncertainty quantification for flow in porous media in hydrogeology and petroleum engineering (Sarma et al., 2005; Li and Zhang, 2007; Li and Zhang, 2009). In the PCM, the dependent random variables are represented by employing the orthogonal polynomial functions (polynomial chaos expansions) as the bases of the random space (Xieet al. 2013).

Polynomial chaos expansions are orthogonal with respect to the specific probability density functions of the input random variables. They are capable of encapsulating information between the input and output random variables by generating accurate "polynomial chaos proxies" with few model evaluations. In addition to polynomial chaos expansion specified for Gaussian random variables, generalized polynomial chaos expansions have been developed for classic probability density functions such as uniform, gamma, beta distributions (Xiu and Karniadakis, 2002) and arbitrary distribution. The key to the PCM is that the simulation values of each parameter (collocation points) at a given order of polynomial chaos expansion can be selected from the roots of the next higher order orthogonal polynomial (Li and Zhang, 2007). Since each distribution has its corresponding orthogonal polynomials, it is obvious that the PCM naturally accounts for parameter distributions. Furthermore, the response surface generated from PCM uses the resulting orthogonal polynomial, which is more robust than traditional response surfaces used in the ED (Xieet al., 2013, Sarmaet al., 2005).

In summary, by returning to the initial discussion about shale gas reservoirs, and given the complex nature of hydraulic fracture networks and extremely low permeability of the matrix, reservoir simulations are the only robust tools, which permit to understand better flow behavior and production mechanisms of shale reservoir in order to evaluate and predict well and reservoir performance. Nevertheless, the semianalytical and analytical solutions cannot capture the very long transient behavior in the matrix blocks of 
extremely low permeability reservoirs. Several approaches were adapted to improve run time of reservoir flow simulation, but they lack the ability to model the transient behavior (Xie et al. 2013, Darishchevet al.2013). On the other hand, the most frequent developed proxy models leaves much to be desired, when it comes to representing numerical reservoir simulation models. Therefore, the need for developing the next generation of proxy model is an extreme need to enable the reservoir modeler to use all capabilities of the developed complex numerical shale simulation model more efficiently.

Data-driven shale proxy model (AI-Based Proxy Model) is our proposed technique as a next generation of proxy models, which will be explained in detail in Chapter 4. It is an Artificial Intelligence-based proxy model that has the capability of reproducing highly accurate well-based and grid-based simulation responses as a function of changes to all the involved input parameters (reservoir characteristics, hydraulic fracture properties, sorption features and operational constraints) in a second. It integrates reservoir engineering and reservoir modeling with machine learning and data mining (Kalantari-Dahaghi et al.2012).

The approximation quality can be controlled by the selection of appropriate activation functions and efficient spatio-temporal database generation. On the other hand, the wide range of alternatives encountered while setting up an artificial neural network (ANN) approximations, can pose a problem for the inexperienced user, which often leads to a categorical rejection of this method. However, if employed properly, ANNs are a suitable approximation technique for high-dimensional and highly nonlinear problems (Kalantari-Dahaghi, Mohaghegh et al 2012).

As presented by Mohagheghat al. (2012), this is not the first time that a technology has been misused, consequently misjudged, and prematurely dismissed. The brief explanation provided in Zubarev's study (2009) on how the Neural Network has been used to build the proxy model, presents ample reasoning on why it did not work. Without going into the details of what was wrong with the way the Neural Network was used in the aforementioned study, it suffices to say that whenever Neural Networks have been used purely as a regression tool it has resulted in disappointing outcomes. Neural Networks should not be used merely as a regression tool, without paying attention that as part of a larger toolset, it attempts to observe, learn, and generalize. This is due to the fact that artificial intelligence and data mining (as an overarching discipline) are far more than regression tools and certain understanding of machine learning activities are required for their effective use and deployment.

Neural networks are the core for the development of Data-driven shale proxy model (surrogate reservoir model) and will be overviewed in the next section of this chapter. 


\subsection{Artificial Intelligence- Neural Network}

As stated before, ANNs are the core for the development of AI-based shale proxy models. An artificial neural network can be defined as an information processing system that has certain performance characteristics similar to biological neural networks. As stated by Fausett (1994), they have been developed as a generalization of mathematical models of human cognition or neural biology, based on the assumptions that:

- Information processing occurs in many simple elements that are called neurons (processing elements).

- Signals are passed between neurons over connection links.

- Each connecting link has an associated weight, which, in a typical neural network, multiplies the signal being transmitted.

- Each neuron applies an activation function (usually non-linear) to its net input to determine its output signal

Figure 2-5 is a schematic diagram of a typical neuron (processing element) in an artificial neural network. Output from other neurons is multiplied by the weight of the connection and enters the neuron as input. Therefore, an artificial neuron has many inputs and only one output. The inputs are summed and subsequently applied to the activation function and the result is the output of the neuron.

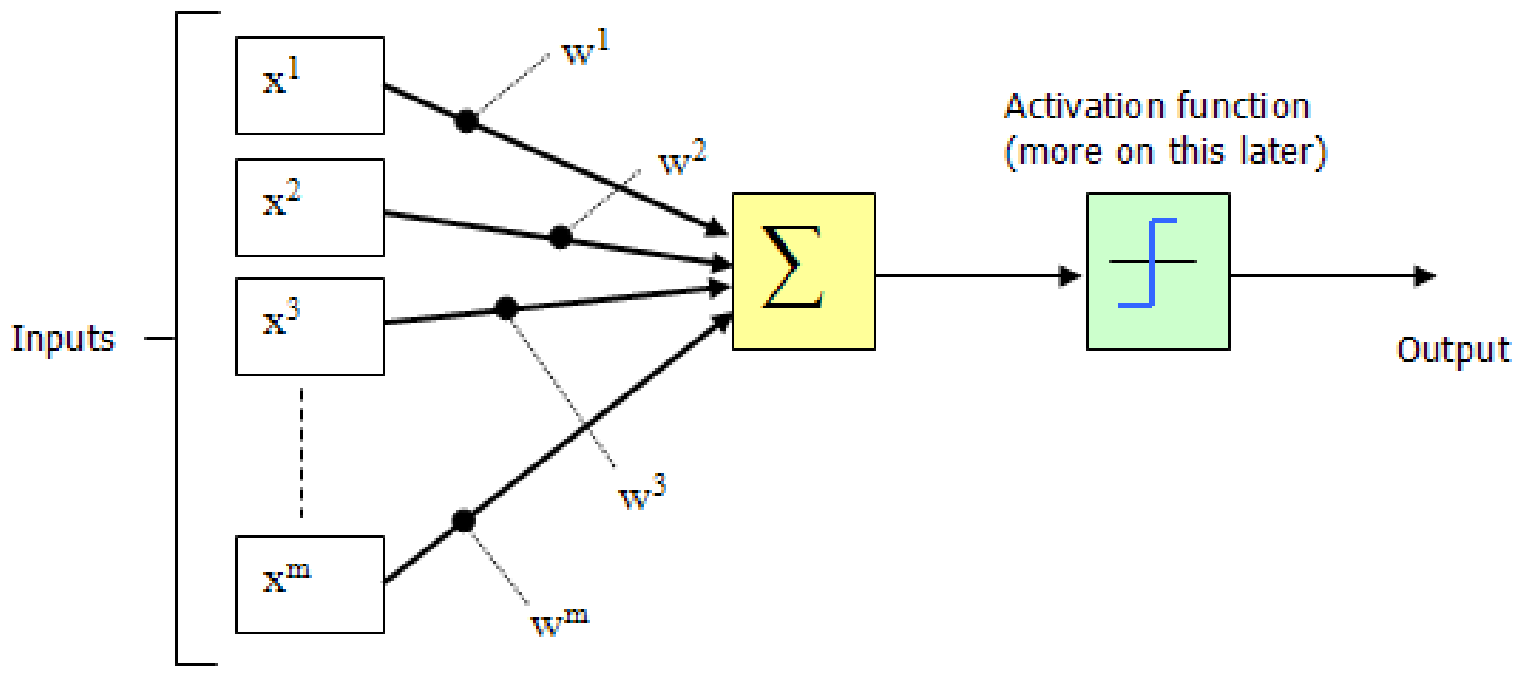

Figure 2-5.Artificial Neuron configuration (Barber 2007)

In an artificial neural network, neurons are grouped into layers. In a multi-layer network there are usually an input layer, one or more hidden layers and an output layer. The number of neurons in the input layer 
corresponds to the number of parameters that are being presented to the network as input. The same is true for the output layer. It should be noted that neural network analysis is not limited to a single output and that neural nets can be trained to build neuro-models with multiple outputs. The neurons in the hidden layer or layers are mainly responsible for feature extraction. They provide increased dimensionality and accommodate tasks such as classification and pattern recognition. Figure 2-6 is a schematic diagram of a fully connected three-layered neural network (Mohaghegh 2000).

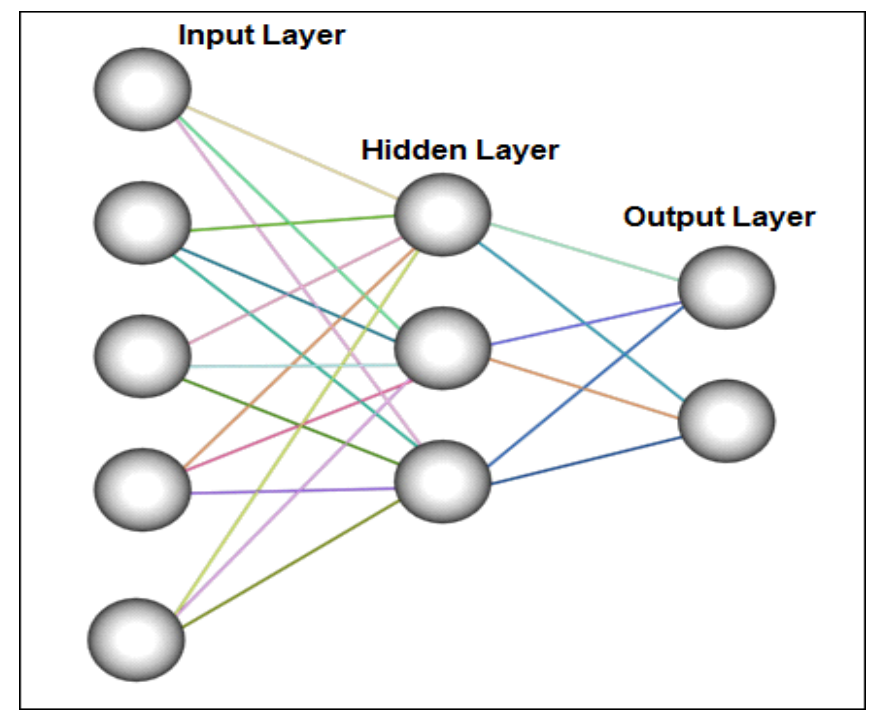

Figure 2-6.Three-layered neural network architecture (Kunzle 2003)

There are many kinds of neural networks. Neural network scientists and practitioners have provided different classifications for neural networks. One of the most popular classifications is based on the training methods. Neural nets can be divided into two major categories based on the training methods, namely supervised and unsupervised neural networks. Unsupervised neural networks, also known as selforganizing maps, are mainly clustering and classification algorithms. They have been used in oil and gas industry to interpret well logs and to identify lithology. They are called unsupervised simply because no feedback is provided to the network. The network is asked to classify the input vectors into groups and clusters. This requires a certain degree of redundancy in the input data and hence the notion that redundancy is knowledge (Barlow 1988, Mohaghegh 2000).

Most of the neural network applications in the oil and gas industry are based on supervised training algorithms. During a supervised training process both input and output are presented to the network to permit learning on a feedback basis. A specific architecture, topology and training algorithm is selected and the network is trained until it converges. 
During the training process, neural network tries to converge to an internal representation of the system behavior. Although by definition neural networks are model-free function approximators, some people choose to call the trained network a neuro-model.

The connections correspond roughly to the axons and synapses in a biological system, and they provide a signal transmission pathway between the nodes. Several layers can be interconnected. The layer that receives the inputs is called the input layer (Figure 2-6). It typically performs no function other than the buffering of the input signal. The network outputs are generated from the output layer. Any other layers are called hidden layers because they are internal to the network and have no direct contact with the external environment. Sometimes they are likened to a "black box" within the network system. However, just because they are not immediately visible does not mean that one cannot examine the function of those layers. There may be zero to several hidden layers. In a fully connected network, every output from one layer is passed along to every node in the next layer (Mohaghegh 2000).

In a typical neural data processing procedure, the database is divided into three separate portions called training, calibration and verification sets. The training set is used to develop the desired network. In this process (depending on the paradigm that is being used), the desired output in the training set is used to help the network adjust the weights between its neurons or processing elements. During the training process the question arises as when to stop the training. How many times should the network go through the data in the training set in order to learn the system behavior? When should the training stop? These are legitimate questions, since a network can be over trained. In the neural network related literature overtraining is also referred to as memorization. Once the network memorizes a data set, it would be incapable of generalization. It will fit the training data set quite accurately, but suffers in generalization. Performance of an over-trained neural network is similar to a complex non-linear regression analysis.

Memorization and over-training is applicable to those networks that are historically among the most popular ones for engineering problem solving. These include back-propagation networks that use an iterative process during the training. In order to avoid over training or memorization, it is a common practice to stop the training process every so often and apply the network to the calibration data set. Since the output of the calibration data set is not presented to the network, one can evaluate network's generalization capabilities by how well it predicts the calibration set's output. Once the training process is completed successfully, the network is applied to the verification data set.

During the training process, each artificial neuron (processing element) handles several basic functions. First, it evaluates input signals and determines the strength of each one. Second, it calculates a total for 
the combined input signals and compares that total to some threshold level. Finally, it determines what the output should be. The transformation of the input to output - within a neuron - takes place using an activation (transfer) function. Sigmoid and Hyperbolic Tangent are two of the commonly used activation (transfer) functions. Sigmoid transfer function is referred to the non-linear curved S-shape function that is the most common type of transfer function used to construct the neural networks. It is mathematically well behaved, differentiable and strictly increasing function (Chakraborty 2010). A sigmoidal transfer function can be written in the form of:

$$
f(y)=\frac{1}{1+e^{-\alpha y}}
$$

The sigmoid function is achieved using exponential equation and it gives the scaled outputs in the range between 0 and 1 . By varying shape parameter, $\alpha$, different shapes of the function can be obtained which adjusts the abruptness of the function. The sigmoid function has another useful characteristic that its derivative is easily expressed in terms of its output as following;

$$
f^{\prime}(y)=f(y)(1-f(y)
$$

Similar to sigmoid function, hyperbolic tangent function is a nonlinear transfer function frequently used in the training of ANNs. The difference from sigmoid is that the output from this function is in the ranges of -1 and 1 and it also differentiable anywhere. Because of this, greater numeric range the hyperbolic tangent function is often used in place of the sigmoid function (Chakraborty 2010). Hyperbolic tangent function is expressed in the form of:

$$
f(y)=\frac{e^{y}-e^{-y}}{e^{y}+e^{-y}}
$$

All the inputs come into a processing element simultaneously. In response, neuron either "fires" or "doesn't fire", depending on some threshold level. The neuron will be allowed a single output signal, just as in a biological neuron - many inputs, one output. In addition, just as things other than inputs affect real neurons, some networks provide a mechanism for other influences. Sometimes this extra input is called a bias term, or a forcing term. It could also be a forgetting term, when a system needs to unlearn something (Nelson et al.1990).

Initially each input is assigned a random relative weight (in some advanced applications - based on the experience of the practitioner - the relative weight assigned initially may not be random). During the 
training process the weight of the inputs is adjusted. The weight of the input represents the strength of its connection to the neuron in the next layer. The weight of the connection will affect the impact and the influence of that input. This is similar to the varying synaptic strengths of biological neurons. Some inputs are more important than others in the way they combine to produce an impulse. Weights are adaptive coefficients within the network that determine the intensity of the input signal. The initial weight for a processing element could be modified in response to various inputs and according to the network's own rules for modification.

Mathematically, the inputs and the weights on the inputs can be treated as vectors, such as $I_{1}, I_{2} \ldots I_{n}$ for inputs and $\mathrm{W}_{1}, \mathrm{~W}_{2} \ldots \mathrm{W}_{\mathrm{n}}$ for weights. The total input signal is the dot, or inner, product of the two vectors. Geometrically, the inner product of two vectors can be considered a measure of their similarity. The inner product is at its maximum if the vectors point in the same direction. If the vectors point in opposite directions (180 degrees), their inner product is at its minimum. Signals coming into a neuron can be positive (excitatory) or negative (inhibitory). A positive input promotes the firing of the processing element, whereas a negative input tends to keep the processing element from firing. During the training process some local memory can be attached to the processing element to store the results (weights) of previous computations. Training is accomplished by modification of the weights on a continuous basis until convergence is reached. The ability to change the weights allows the network to modify its behavior in response to its inputs, or to learn. For example, suppose a network identifies a production well as "an injection well". On successive iterations (training), connection weights that respond correctly to a production well are strengthened and those that respond to others, such as an injection well, are weakened until they fall below the threshold level and the correct recognition of the well is achieved (Mohaghegh 2000).

There are several types of ANNs in literature. For the purpose of this study Feed-Forward Backpropagation network that is one of the most commonly used supervised training algorithms is used. In this $\mathrm{NN}$ the artificial neurons are organized in layers, and send their signals "forward", and then the errors are propagated backwards. In the back propagation algorithm, the network output is compared with the desired output - which is part of the training data set, and the difference (error) is propagated backward through the network. During this back propagation of error, the weights of the connections between neurons are adjusted. This process is continued in an iterative manner. The network converges when its output is within acceptable proximity of the desired output. 


\subsection{Summary}

In this chapter, the current stage of shale numerical simulation is discussed and the weak and strong points of the current technique are highlighted. No matter which simulation modeling technique (Dual porosity with explicit hydraulic fractures, SRV, wiremesh etc.) be used for modeling shale gas production performance, model setup and development specially for the EHF technique is long and the computational overhead can prove to be impractical once the modeling goes beyond a single pad. On the other hand, the most frequent developed proxy models leaves much to be desired when it comes to representing numerical reservoir simulation models therefore the need for developing the next generation of proxy model is an extreme need. Moreover, the possibility of injecting $\mathrm{CO}_{2}$ for enhancing methane recovery and $\mathrm{CO}_{2}$ storage is discussed and an overview of different types of ANN and its functionality is presented. 


\section{CHAPTER 3 SHALE GAS NUMERICAL SIMULATION DEVELOPMENT- INTEGRATED WORKFLOW}

\subsection{Summary}

In this chapter, a detailed procedure for developing an integrated workflow, which demonstrates a quantitative platform to model shale gas production through capturing the essential characteristics of shale gas reservoirs is discussed (Figure 3-1). The workflow entails three main steps procedure. First, systematic procedure to develop a base geological model using well logs is discussed. Second, the procedure for DFN development, up-scaling and modeling the impact of the hydraulic fracture and incorporation of fracture characteristics in the geological model are explained. Third, the chapter is finished by detailing the history matching procedure and forecasting the production for the next 100 years.

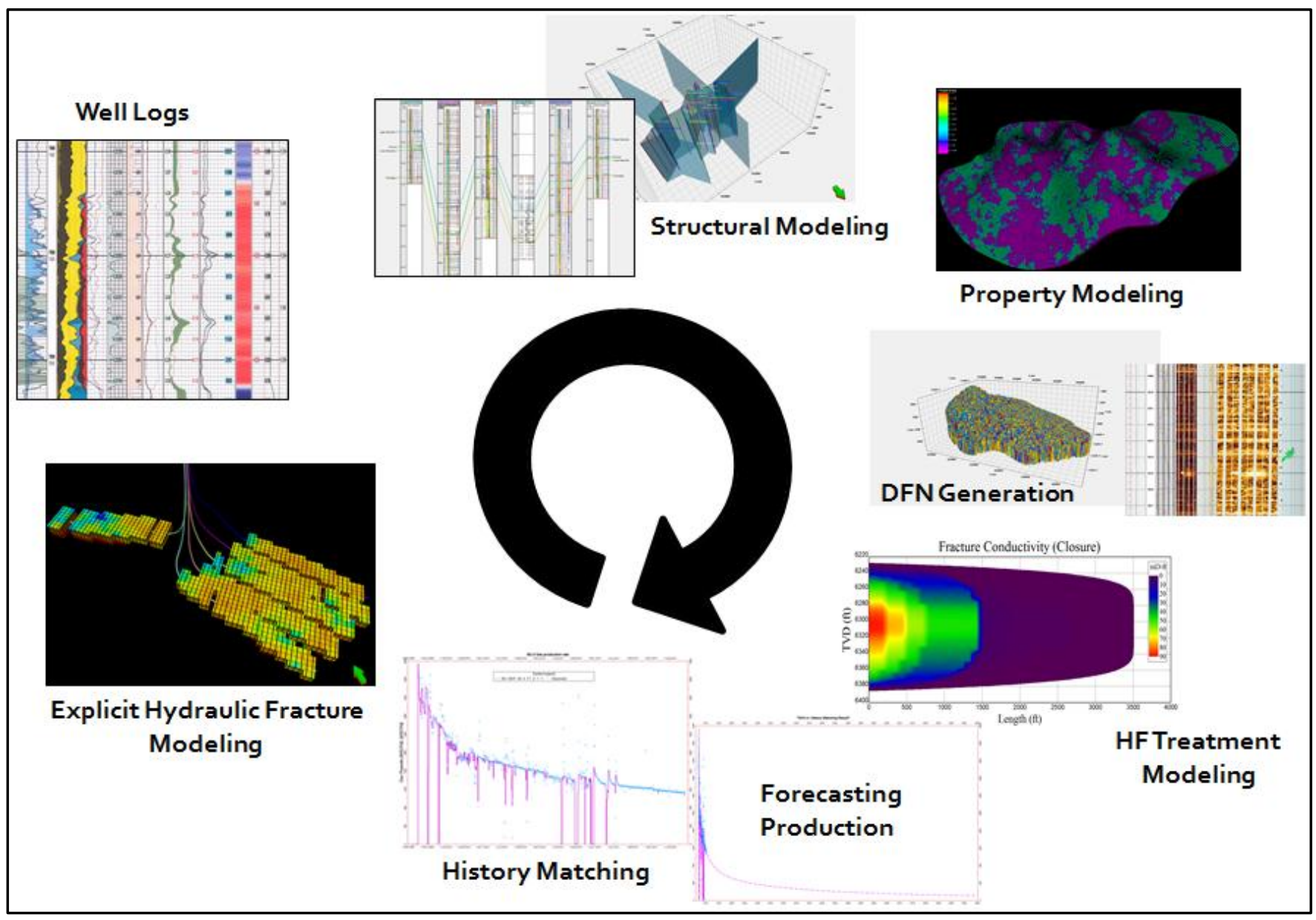

Figure 3-1-Marcellus shale geomodeling to simulation workflow 


\subsection{Base Geological Model}

Modeling and simulation of shale gas reservoir are challenging due to complex nature, strong heterogeneous and anisotropic system, different reservoir behavior, multiple gas-storage mechanisms and unique attributes that control productivity, which is vastly different from conventional reservoirs. Therefore, building a general and practical workflow is critical in order to capture all aspects of shale characteristics and to obtain a clear understanding and an accurate description of the reservoir.

As in all serious shale reservoir simulation and modeling exercises, developing a geological model is a necessary step in the numerical modeling of production from shale. During this step all the geological, petrophysical and geophysical information available to the modeling team is used to develop a reasonably detailed geological model. Even for a single well model, this process may generate a detail multi-million grid block geological model. Usually data from all the available wells are used to generate the structural map and volume that is then populated with appropriate data based on availability. This process is usually performed using a geological modeling software application, several of which are currently available in the market and are extensively used during the modeling process.

Inclusion of Discrete Fracture Network (DFN) in the modeling process is performed during this step. The common approach is to develop the DFN using statistical means and then use analytical or numerical techniques to incorporate the impact of the developed DFN into the existing grid block system that is generated during the construction of the Geo-cellular model.

\subsubsection{Data collection}

Information from 77 Marcellus shale gas wells in the Southwestern Pennsylvania with a total number of 652 stages of hydraulic fracture and 1893 clusters in the area of about 53,241 acres, are used to perform the analysis in this study.

Developing a detailed geological model in the vicinity of the well, requires the integration of wellbore measurements (e.g. core data, well logs), which provide important details of the lateral heterogeneity and variation of reservoir and rock properties.

As shown in Table 3-1, all data items for integrated shale gas study and simulation are categorized into six groups:1) Wells location and trajectories, 2) Static data, 3) Well logs, 4) Completion information, 5) hydraulic fracturing data (Hard data) and 6) Production data and operational constraints and are shown. Additionally, some other data including PVT, Gas composition, Rock physics functions (relative 
permeability and capillary pressure), Isotherms and diffusion coefficients for each component (i.e. $\mathrm{CH}_{4}$ and $\mathrm{CO}_{2}$ ) are used for performing the simulation.

Table 3-1Integrated Marcellus Shale Gas Study -Data list

\begin{tabular}{|c|c|c|}
\hline Easting & \multirow{6}{*}{ 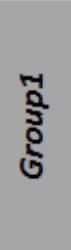 } & \multirow{6}{*}{ 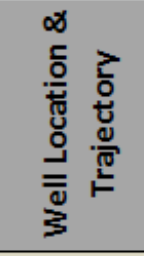 } \\
\hline Northing & & \\
\hline $\mathrm{MD}$ (End) & & \\
\hline TVD (End) & & \\
\hline Azimuth (End) & & \\
\hline Inclination (End) & & \\
\hline Upper Marcellus -Porosity \% & \multirow{5}{*}{$\begin{array}{l}r 1 \\
\text { r } \\
0 \\
3 \\
0 \\
0\end{array}$} & \multirow{10}{*}{ 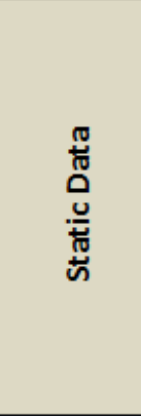 } \\
\hline Upper Marcellus -Permeability (md) & & \\
\hline Upper Marcellus -Gross Thickness(ft) & & \\
\hline Upper Marcellus -NTG & & \\
\hline Upper Marcellus -TOC \% & & \\
\hline Lower Marcellus -Porosity\% & \multirow{5}{*}{$\begin{array}{l}\text { N } \\
\text { v } \\
\frac{0}{3} \\
0 \\
\text { ல }\end{array}$} & \\
\hline Lower Marcellus -Permeability(md) & & \\
\hline Lower Marcellus -Gross Thickness (ft) & & \\
\hline Lower Marcellus -NTG & & \\
\hline Lower Marcellus -TOC \% & & \\
\hline Conventional Logs (Gamma ray, resistivity, density etc.) & \multirow{4}{*}{$\begin{array}{l}m \\
\stackrel{2}{3} \\
0 \\
\text { ல }\end{array}$} & \multirow{4}{*}{ 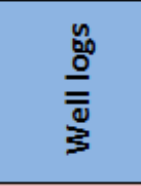 } \\
\hline Geomechanical Logs (Sh min ,Young's Modulus, Poisson's Ratio, etc) & & \\
\hline FMI logs ( Dip angle andDip Azimuth) & & \\
\hline Producttion Logging tool (PLT) & & \\
\hline Perforation Intervals & \multirow{4}{*}{ 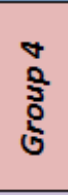 } & \multirow{4}{*}{$\frac{\text { 음 }}{\frac{0}{0}}$} \\
\hline HF Clusters coordinate & & \\
\hline Number of Clusters per Stage & & \\
\hline Shot Density (shots/ft) & & \\
\hline Average Injection Pressure per Stage (psi) & \multirow{6}{*}{$\begin{array}{l}\text { no } \\
0 \\
0 \\
0 \\
6\end{array}$} & \multirow{6}{*}{ 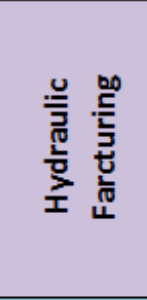 } \\
\hline Average Injection Rate Per Stage (bbl/min) & & \\
\hline Total clean Volume per Stage(bbl) & & \\
\hline Total Slurry Volume per Stage(bbl) & & \\
\hline Maximum Proppant Concentration per Stage(lb/gal) & & \\
\hline Total Proppant per stage(lb) & & \\
\hline & \multirow{3}{*}{$\begin{array}{l}6 \\
0 \\
5 \\
0 \\
6\end{array}$} & \multirow{3}{*}{ 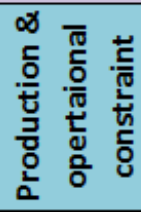 } \\
\hline Daily gas production (MScf/day) & & \\
\hline Calculated Flowing Bottob-hole Pressure from well head pressure(psia) & & \\
\hline
\end{tabular}

\subsubsection{Structural Modeling}

A structural model can be built with defined significant stratigraphic horizons, lithological zones and lease line boundary and proper layering can be achieved by taking into account the reservoir simulation cell geometry requirement. As shown in Figure 3-2, three distinct zones can be identified based on 
gamma ray log. It should be noted that upper and lower Marcellus are both considered as reservoir layers and Purcell, which is a thin limestone layer, is treated as non-reservoir layer.

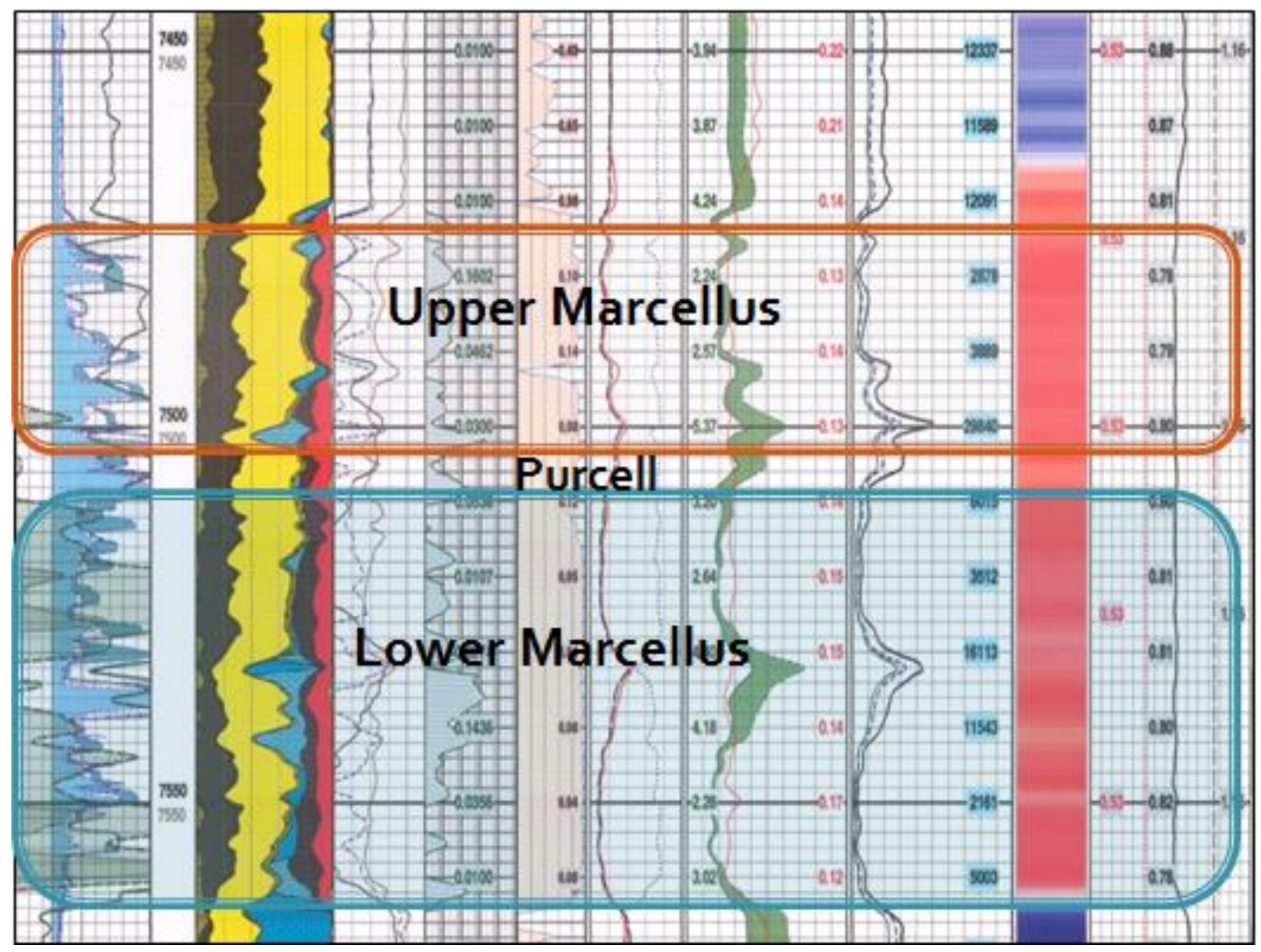

Figure 3-2.Marcellus Shale well log

Table 3-2 shows the minimum, maximum and average thickness of Upper, Lower Marcellus and Purcell in this study based on log interpretation.

Table 3-2-Marcellus shale thickness

\begin{tabular}{cccc}
\hline & Gross Thickness Min. (ft.) & Gross Thickness Max.(ft.) & Gross Thickness Mean(ft.) \\
\hline Upper Marcellus & 20 & 85 & 33 \\
Purcell & 2 & 15 & 8 \\
Lower Marcellus & 35 & 134 & 62 \\
\hline
\end{tabular}

In order to construct the structure of the reservoir, identifying the top and bottom of each layer is the minimum requirement. Therefore, 38 logs with high quality gamma ray profile are used for top picking. 
Five different cross sections are constructed across the reservoir to include all the wells in the area of study. Figure 3-3 shows the detail of five cross sections in 2D and 3D and one of them is illustrated in detail in Figure 3-4. Moreover, top of Upper Marcellus, Purcell, Lower Marcellus and Onondaga formations can be seen in this figure.

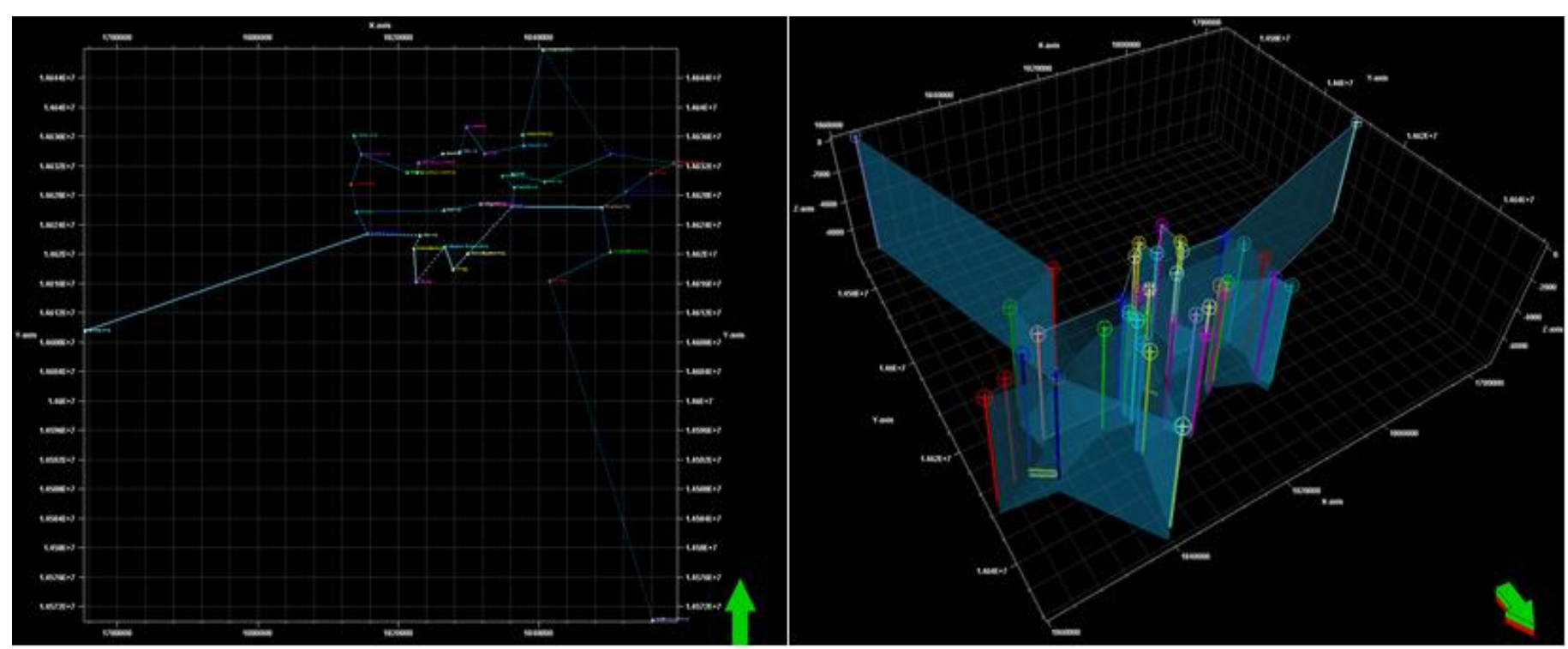

Figure 3-3.2D and 3D representation of Five cross-sections across the reservoir (Left to right)

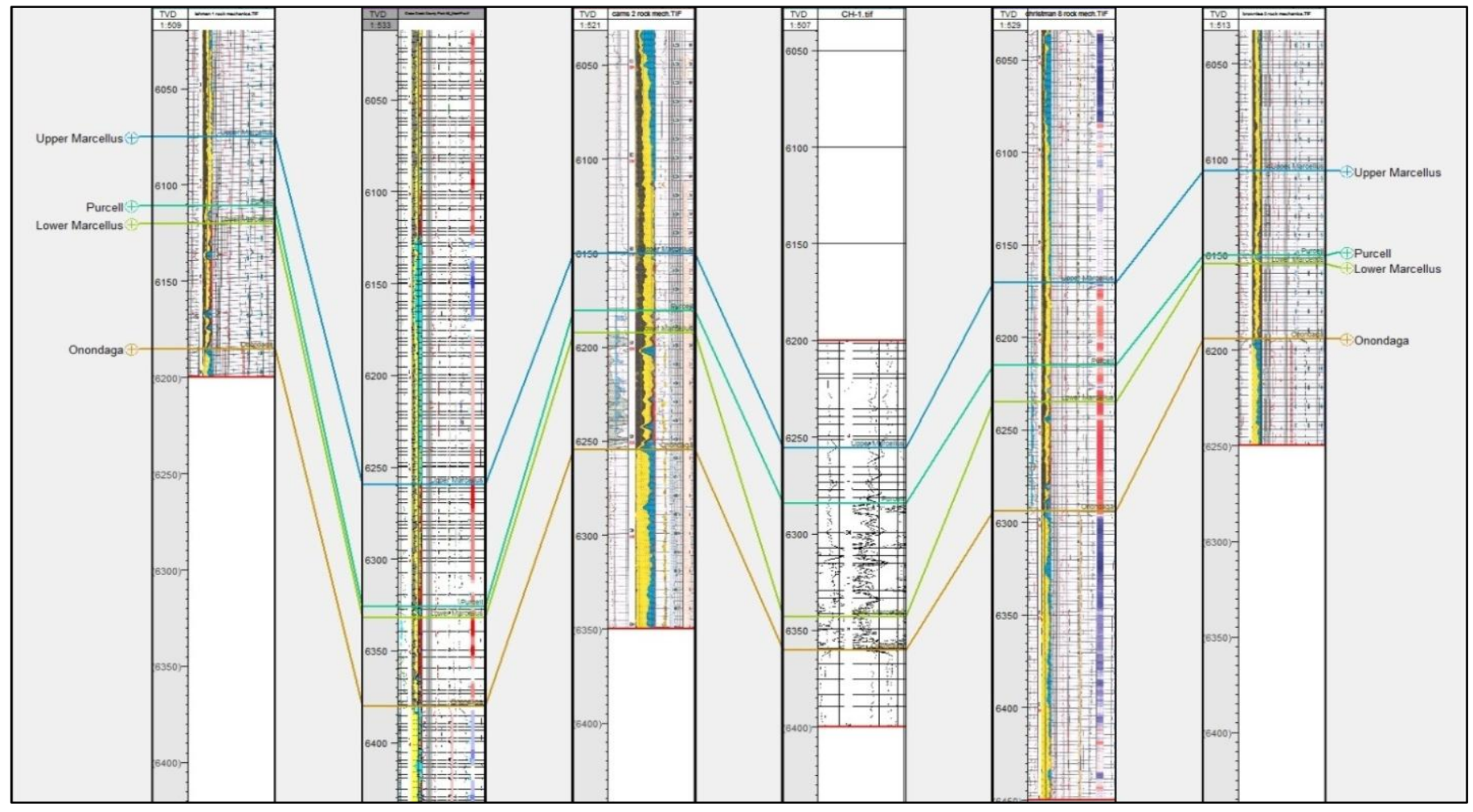

Figure 3-4.Cross Section /Top picking example in one of the cross sections 
After identifying the tops for each geological layer (rock type), the surfaces of each layer is constructed. Figure 3-5 shows the top structure for Upper Marcellus as an example.

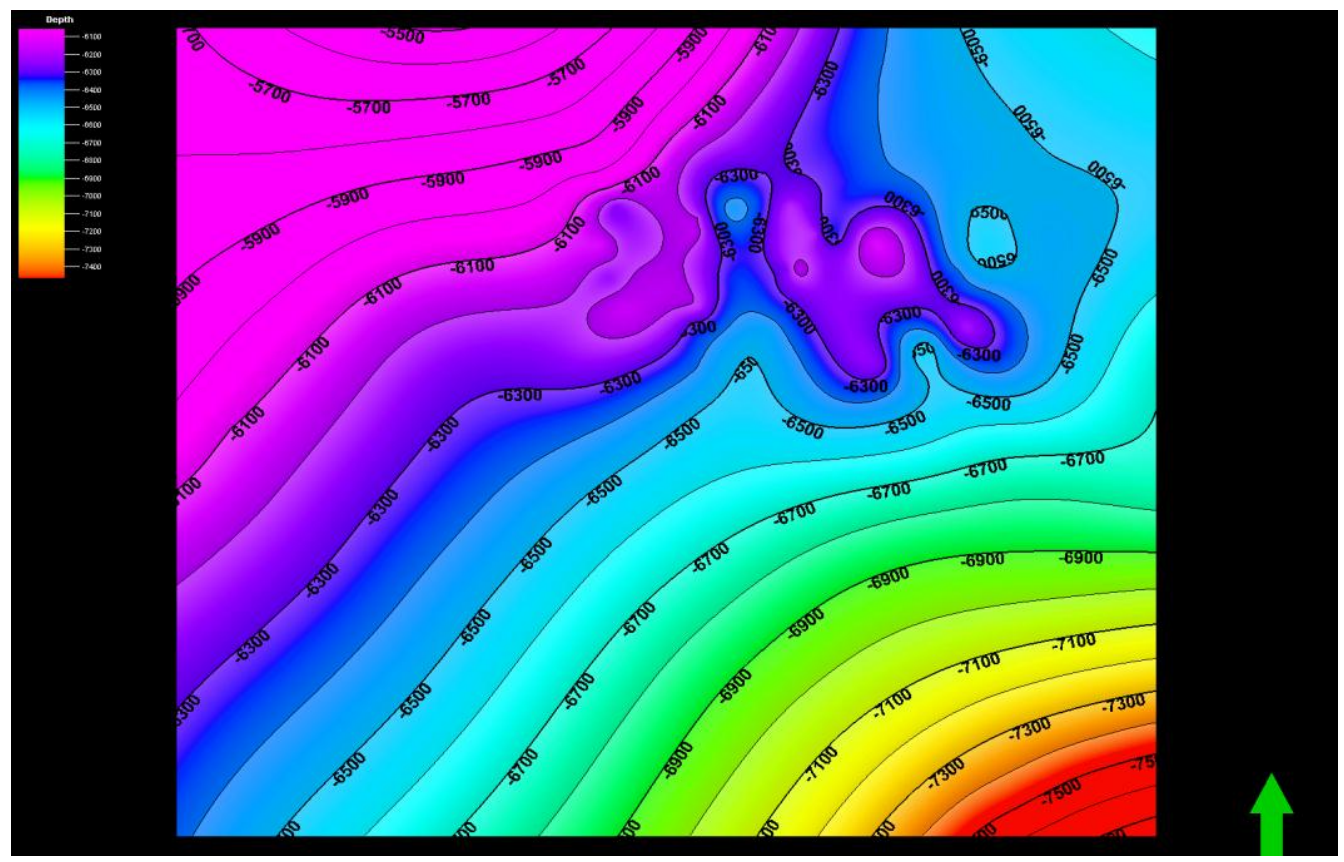

Figure 3-5Upper Marcellus Top Structure

After generating the surface for each layer, the target area, which has more population of wells, is identified (Figure 3-6) and around 1.16 million grid blocks (100*100 ft.) are generated to cover the study area (Figure 3-7).

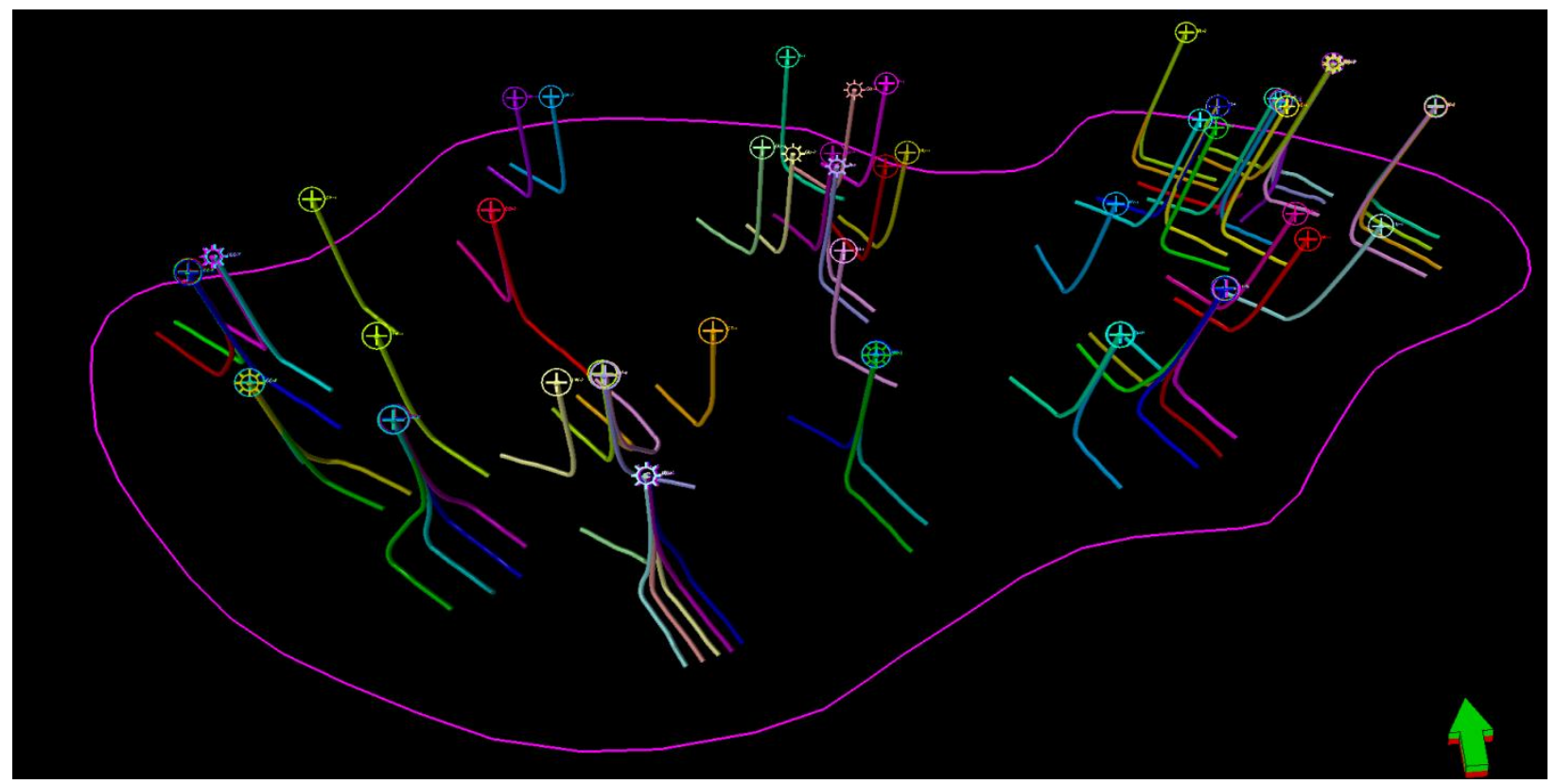

Figure 3-6.Distribution of 76 wells in the reservoir and reservoir boundary 


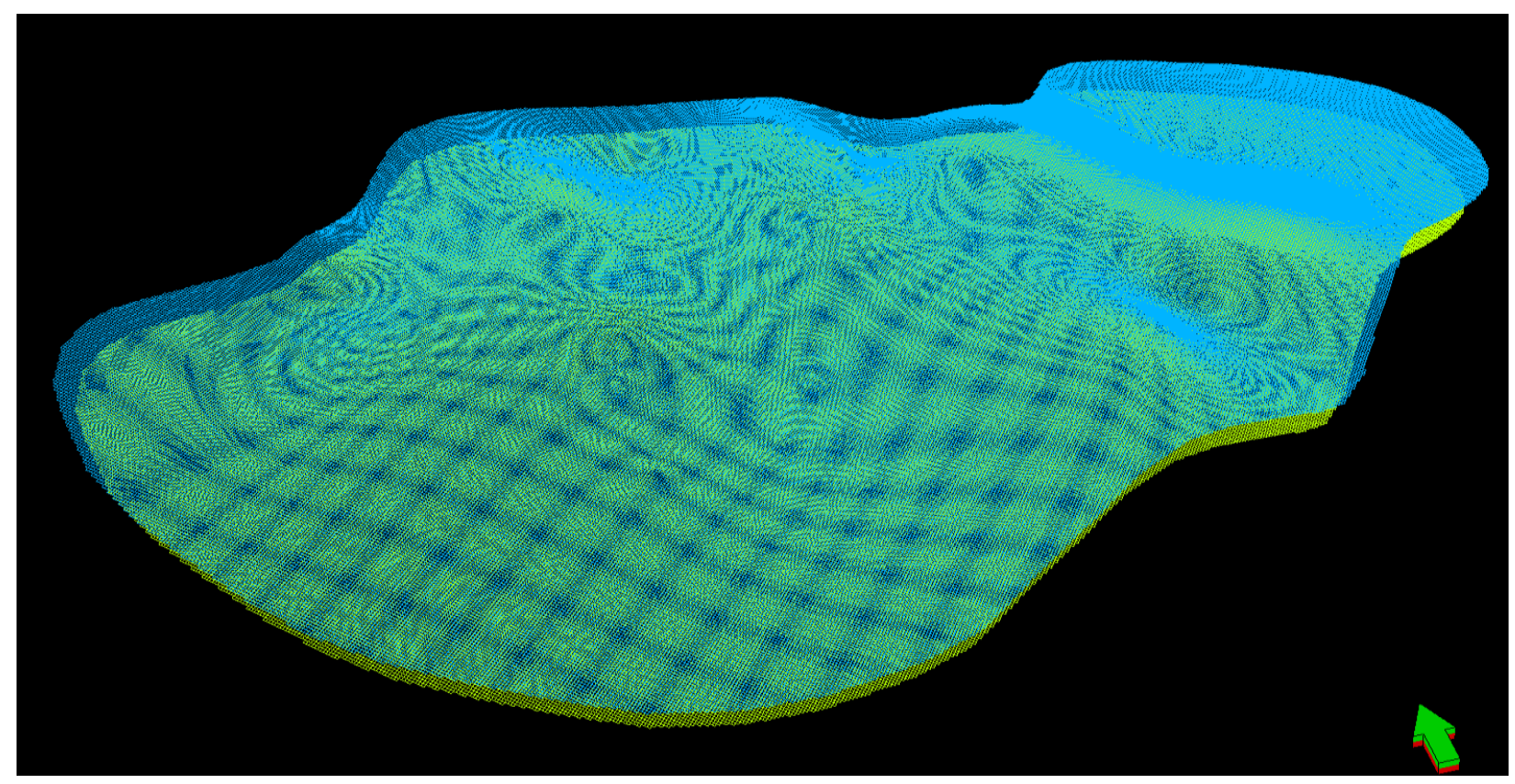

Figure 3-7.3D Grid skeleton representation for 1.16 million grid blocks

\subsubsection{Property Modeling}

Property modeling is the process of filling cells of the grid with discrete or continues properties. The objective is to use all geological information available to build a realistic property model. Therefore, Sequential Gaussian Simulation(SGS) is used to generate the gesostatistical distribution of all the properties including: matrix porosity, matrix permeability, net to gross thickness, TOC and also geomechanical properties such as Bulk Modulus, Shear Modulus and Young's Modulus, Poisson's ratio and Minimum horizontal stress for all three rock types.

Figure 3-8 illustrates the distribution of matrix porosity, NTG and matrix permeability for Upper and Lower Marcellus as an example. In this figure, the red and purple color represents the maximum and minimum value of the parameter in the map, respectively.

Marcellus shale is extremely variable in thickness, ranging from a few feet to more than 250 feet in thickness, and generally becomes thicker to the east. Due to different well trajectory (deviation type), landing targets and completed stages, the pay zone is fully or partially accessible. The Net to Gross ratio ranges from 0.74 to 0.985 . Matrix porosity and matrix permeability are changing from 5 to $12.5 \%$ and 0.00018 to $0.0009 \mathrm{md}$. In general, Lower Marcellus has higher quality than Upper Marcellus in terms of matrix porosity, matrix permeability, NTG and TOC. 

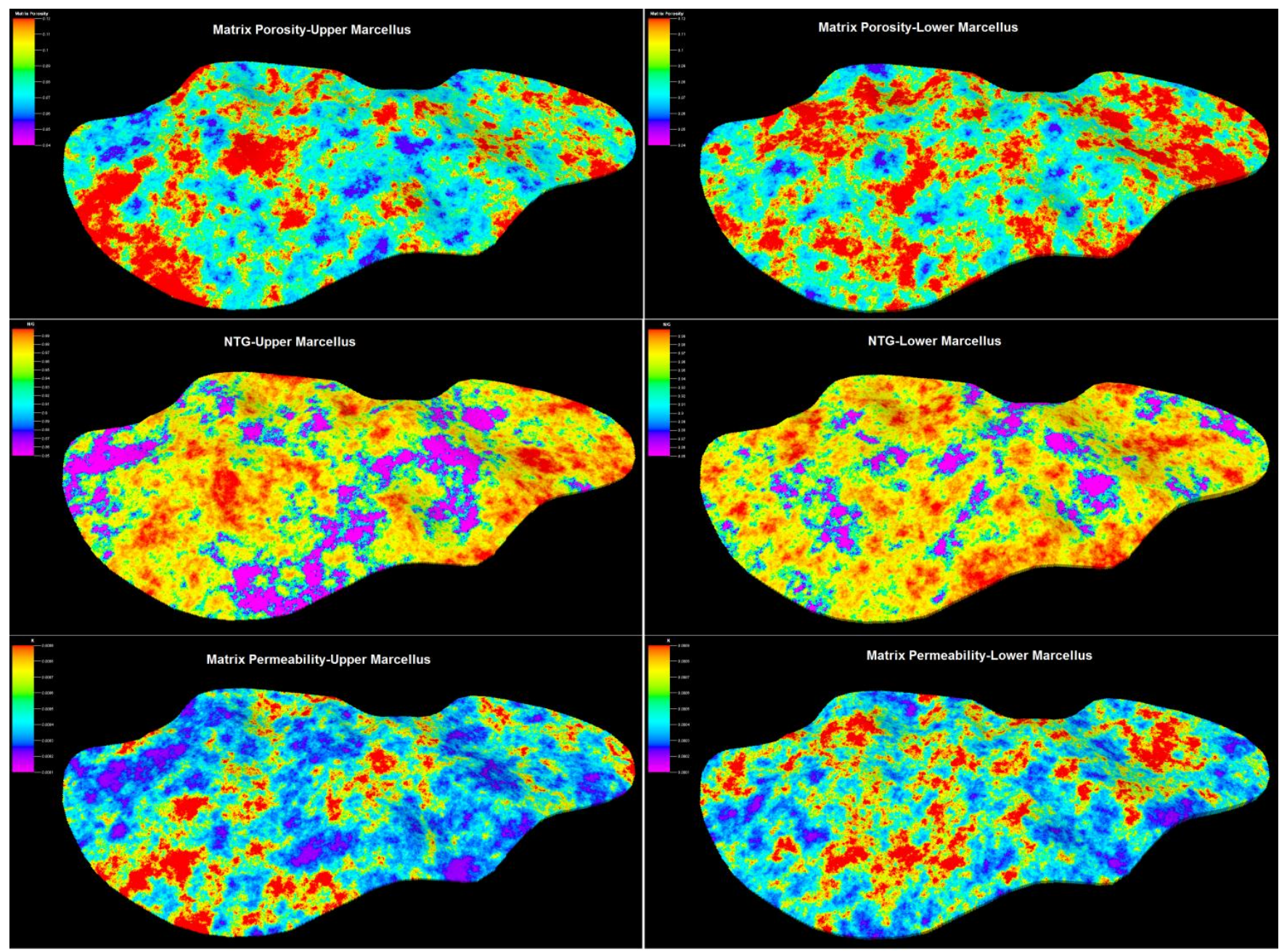

Figure 3-8.Distribution of Matrix porosity, Net to Gross thickness and Matrix permeability in Upper and Lower Marcellus

Geomechanics plays a very important role in the hydraulic fracture generation, propagation and effective proppant placement and consequently, production performance of a horizontal shale gas well. In this study, 30 gemochenaical logs are interpreted to extract very important gemochenaical properties such as Bulk Modulus, Shear Modulus and Young's Modulus, Poisson's ratio and Minimum horizontal stress for all three layers (UM, LM and Purcell). Figure 3-10 illustrates the distribution of Minimum horizontal stress, Young's Modulus, Poisson's ratio as an example.

As a proven fact, a horizontal well should be drilled parallel to minimum horizontal stress, while the hydraulic fractures tend to initiate parallel to maximum horizontal stress. Rock mechanical studies show that, any increase in minimum horizontal stress causes the decrease in created hydraulic fracture width and results in lower performance of hydraulic fracture (HF). As a result, by looking at minimum horizontal stress distribution in the reservoir, the area of having higher minimum horizontal stress is less 
favorable for creating wide and more conductive hydraulic fractures (Figure 3-10). Minimum horizontal stress gradient ranges from a minimum of 0.51 up to a maximum of $0.88 \mathrm{psi} / \mathrm{ft}$. in the area of study.

Young's modulus is the other critical elastic attributes for shale gas reservoir characterization, which indicate the resistance of the rock to elastic deformation under pressure, i.e. the less young's modulus, the more flexible the rock could be. Large young's modulus makes it harder for the fracturing fluid to produce width. This will make the fracture thinner, higher and longer. As stated by Cipolla (2010), Marcellus shale is amidst those shale reservoirs with lower Young's modulus ( $\left.\mathrm{E} \approx 2.5 \times 10^{6} \mathrm{psi}\right)$ and therefore, the higher closure stress on the fracture network could significantly reduce the production. Although the impact of increasing closure stress on the fracture network can be severe for lower-modulus rock, the effects may not be evident during the initial 1-2 years of production. As shown in Figure 3-9, this property ranges from $1.8 \mathrm{E}+6$ to a maximum up to $5 \mathrm{E}+6 \mathrm{psi}$ in some part of the reservoir.
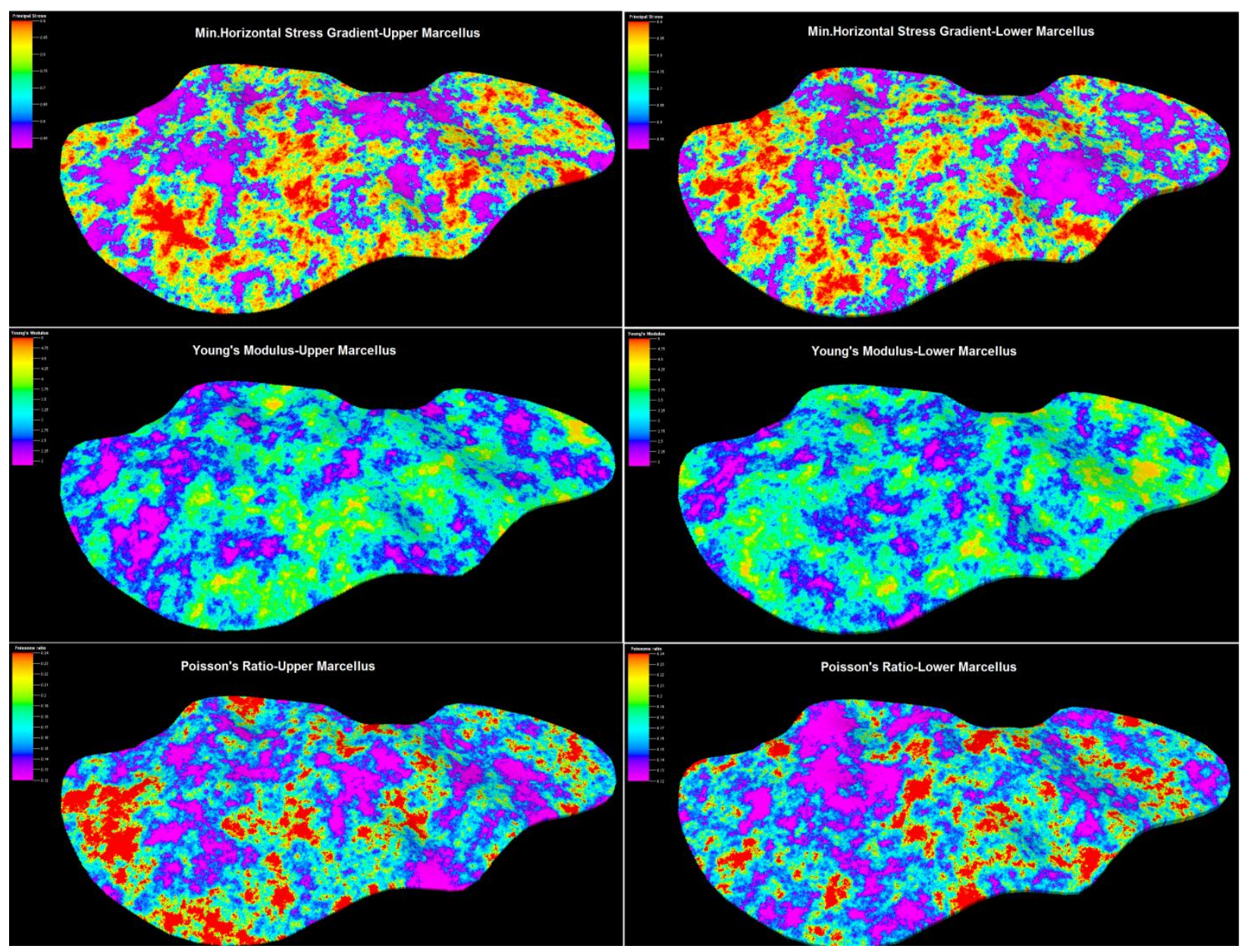

Figure 3-10. Distribution of Min. Horizontal stress gradient, Young's modulus and Poisson's ratio in Upper and Lower Marcellus 
Poisson's ratio is the other important elastic parameter. Generally, zones of high Poisson's ratio and low young's modulus indicate the ductile behavior of rocks so that they can be avoided during hydraulic fracturing. This parameter varies from 0.1 to 0.24 .

\subsubsection{Natural Fracture Modeling -DFN Generation}

The most common technique for modeling Discrete Natural Fracture (DNF) network is to generate them stochastically. Using Borehole Image Logs (e.g. FMI), some of the initial characteristics of the DNF can be estimated and used in their stochastic generations. Parameters such as fracture point's dip angle, dip azimuth, averages for fracture length, aperture, density of center points are among those that need to be provided (guessed or estimated) so that the stochastic algorithms can generate a Discrete Natural Fracture Network (DFN).

FMI-interpreted fractures are subject to easier opening by hydraulic fracturing than virgin shale rock. From the practical point of view, all types of interpreted fractures can be considered as constituting a "natural" fracture network that partially dominates hydraulic fracture network intensity and distribution. Additional important factors, such as in-situ stress field and geomechanical properties, have great impact on generation of complex fracture systems.

To continue with the workflow, 21 FMI logs are used to model natural fracture distribution in the Marcellus shale. FMI interpreted fractures are classified, analyzed and two important properties of dip angle and dip azimuth are extracted in several depths for each well. Figure 3-11 shows 3D display of Dip angle point data in each layer (UM, Purcell and LM) for one of the wells as an example. The fracture data points are then used to generate Intensity $\log$, which is used as fracture density volume for the generation of DFN.

In addition, fracture points for all 21 wells are plotted on the stereonet (Figure 3-12) that shows the direction of main natural fracture set from NNE to SSW that is parallel to maximum horizontal stress.

With proper property drivers, fracture intensity 3D distributions can be achieved, mostly with stochastic simulations .With given well control fracture dips and azimuths input as constants, 2D or 3D properties, and specific fracture geometry specifications, 3D discrete fracture network (DFN) is generated (Figure 3-13). 

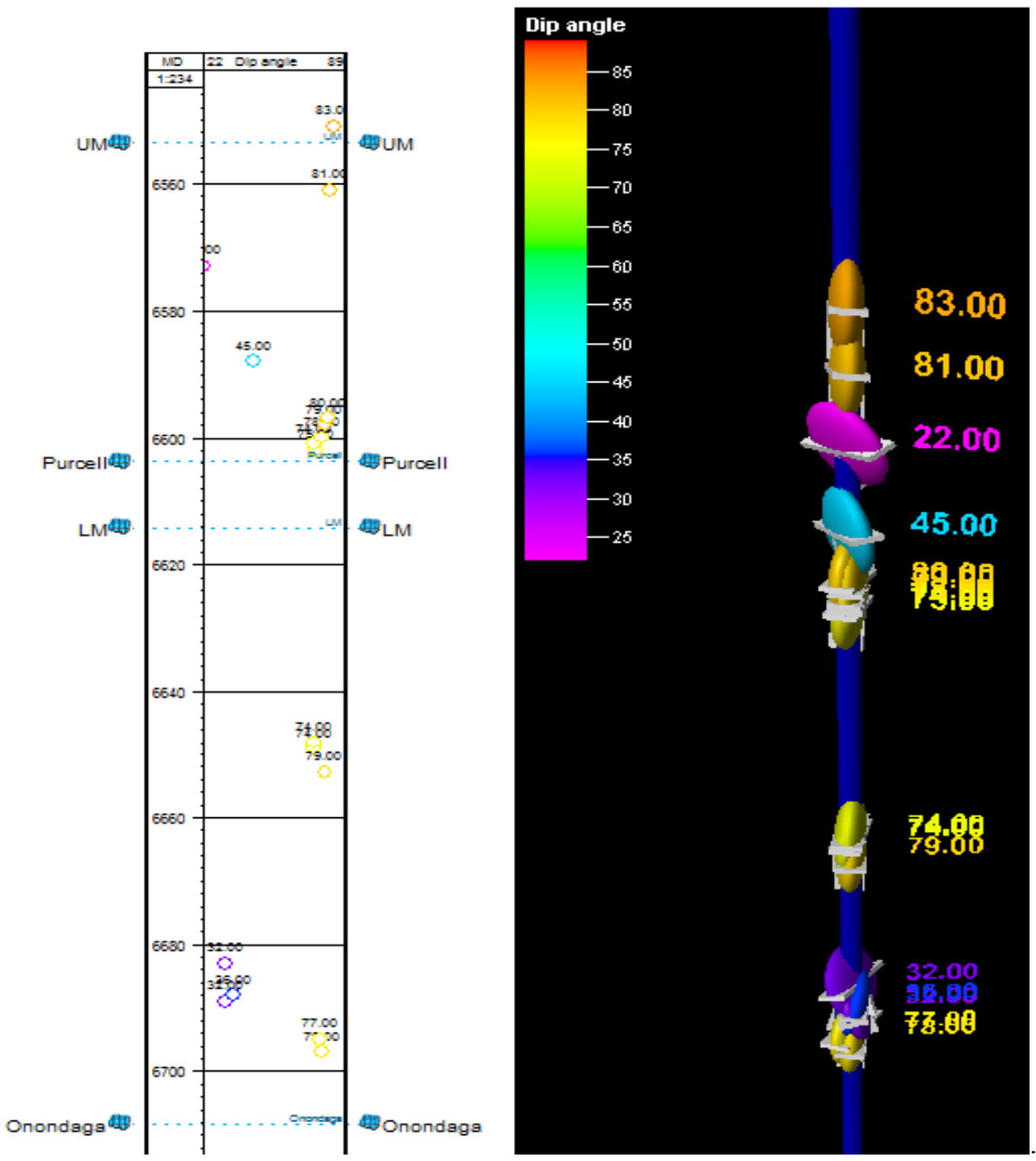

Figure 3-11.3D display of Dip angle point data for one of the wells.

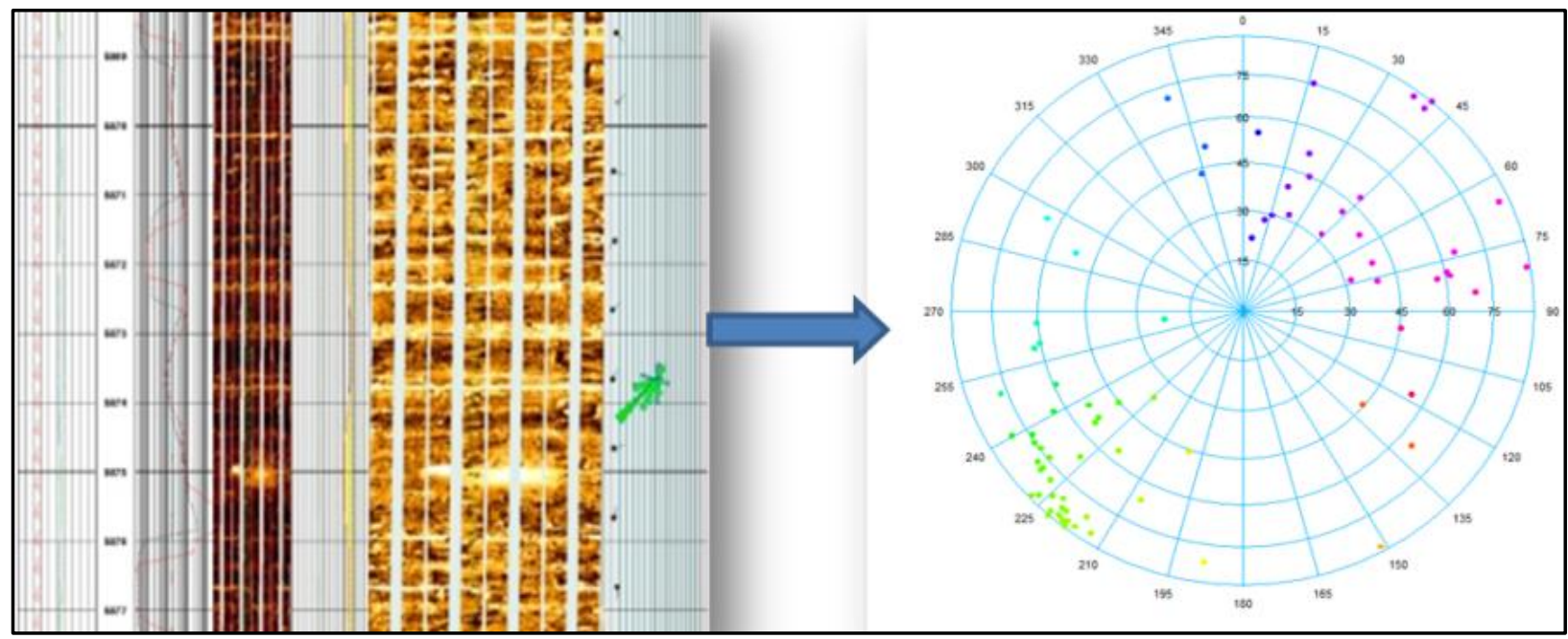

Figure 3-12. FMI log example for one of the wells and fracture point data illustration on stereonet for all the wells. 


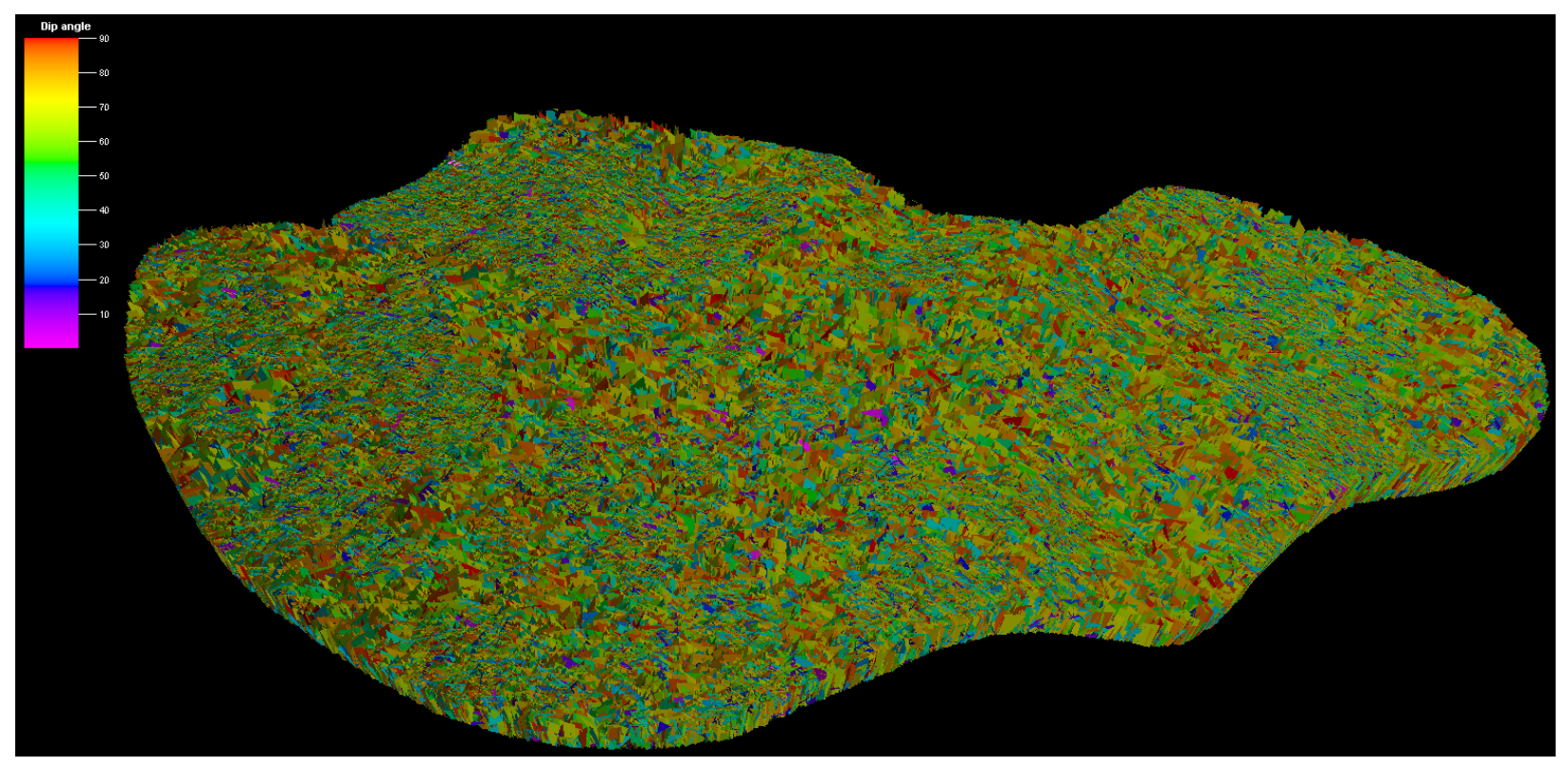

Figure 3-13. Dip angle distribution in generated Discrete Fracture Network (DFN)

One of the difficulties in DFN modeling is the fracture geometry and its distributions. These parameters have to come from relevant and realistic geological studies. Even though may be possible to obtain some references from a fracture intensity property, but the final estimation has to come from production history matching.

A DFN model is made with some fracture network attributes, however, for practical purposes in dynamic reservoir models, these are not useful until upscale them into the required grid properties that can be used for performing dual continuum (dual porosity or dual porosity/dual permeability) flow simulation. The new grid properties are fracture permeability (either diagonal or full tensor), fracture porosity and shape factor (matrix-fracture transfer function). The final properties for each grid will be obtain during the history matching process.

Fracture porosity is calculated simply by:

$$
\emptyset_{f}=\text { Total fracture area } * \text { Aperture/Volume of cell }
$$

The numerically derived expression for sigma (shape) factor in terms of fracture spacing (matrix block size) in $\mathrm{i}, \mathrm{j}$ and $\mathrm{k}$ grid coordinate directions of given cell is as follows:

$$
\sigma=4 *\left(\frac{1}{\mathrm{~L}_{\mathrm{i}}^{2}}+\frac{1}{\mathrm{~L}_{\mathrm{j}}^{2}}+\frac{1}{\mathrm{~L}_{\mathrm{k}}^{2}}\right)
$$


Oda and Flow based methods are two common techniques to upscale the DFN properties in the grid .A brief description of each technique is as follows:

ODA method (Statistical)-This method primarily relies on the geometry and distribution of fractures in each cell to build permeability tensor .It uses a statistical method based on the number and sizes of the fractures in each cell. It is fast but does not take into account the connectivity of fractures and can therefore underestimate fracture permeability when the intensity is low.

Flow-based tensor up scaling -It creates a finite element grid for each grid cell and uses finite element code to run three small-scale flow simulations per coarse cell on the DFN. It is much slower than Oda method, but provides accurate results.

In this study, due to very large reservoir size, Oda method is used to upscale DFN to grid properties. Figure shows the distribution of natural fracture porosity, natural fracture permeability and matrix-fracture transfer function (Sigma).In this figure, the red and purple colors illustrate the maximum and minimum value of the parameter in the map, respectively.

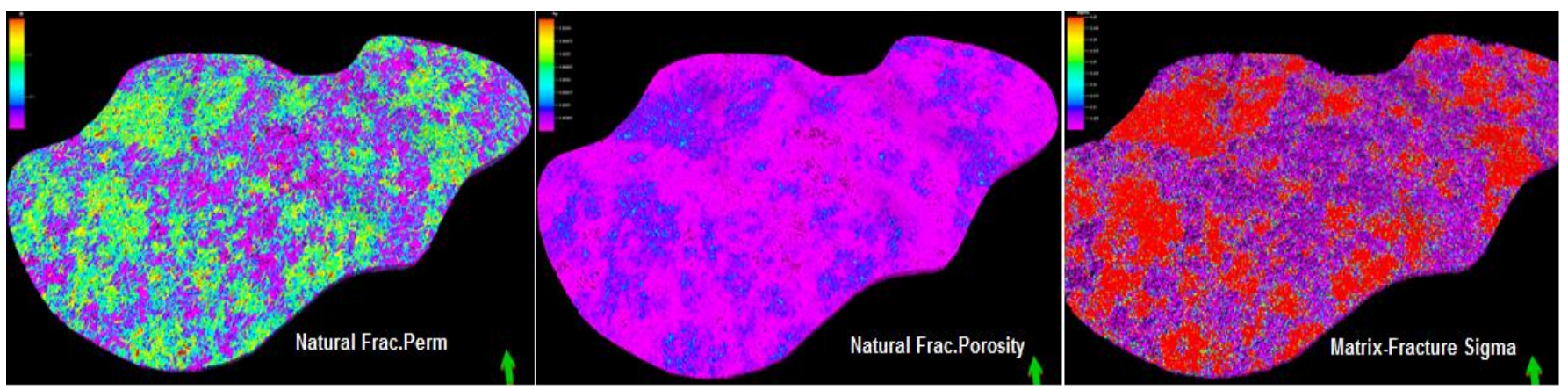

Figure 3-14.FMI log and discrete fracture network

\subsection{Explicit Hydraulic Fracture Modeling (EHF)}

During this step, each cluster of hydraulic fracture is modeled individually using independent hydraulic fracture simulation software applications such as $\mathrm{MFrac}^{\mathrm{TM}}$. These models use the fracturing job characteristics (hard data) such as fluid and proppant amount and rate of injection, fluid loss, well trajectory, perforation, along with some reservoir characteristics and, stresses to calculate the characteristics of an idealized hydraulic fracture. Since these models assume a well-behaved pennyshaped hydraulic fracture, the characteristics they calculate are fracture half-length, fracture height, fracture width, and fracture conductivity. This process is repeated for every single cluster of hydraulic fractures. 
In order to incorporate the hydraulic fracture characteristics into the geological model, first all the wellbores and corresponding completions must be included. Upon inclusion of the well bore, all the calculated hydraulic fracture characteristics are imported into the geological model. This is a rather painstaking process through which the grid system developed during geological modeling is modified in order to be able to accommodate the hydraulic fracture characteristics and properly incorporate the transient flow behavior from the matrix to the fracture. Usually a logarithmic local grid refinement process is required (both horizontally as well as vertically) for this process. The result is a detailed model that includes a large number of grid blocks. When building a model that includes multiple wells in a pad or multiple pads, this process may take a long time.

Due to the detailed nature of the model, the computational cost of such models is too high. This fact makes full-field modeling and history matching of shale assets, impractical. That is the main reason behind the fact that the overwhelming number of numerical simulation studies conducted on shale formations so far, (At the time of writing this dissertation) are single well models. Still, the amount of time it takes to complete all the steps and develop an explicitly hydraulic fracture model and history match gas production from several horizontal wells in a Pad can be quite extensive.

Nevertheless, for the purpose of this research, this process is completed for multiple pads and at the end, due to computational limitation for performing numerical simulation, one of the pads with six horizontal wells with 169 clusters of hydraulic fracture is selected for history matching. This pad is referred to as the WVU pad that is a good representative of the Marcellus asset in the study area, in terms of reservoir characteristic, number stages of hydraulic fracture and the rest of hydraulic fracturing hard data. The comparison of WVU pad with all the wells in terms of hydraulic fracturing hard data is summarized in Table 3-3.

Table 3-3-Hydraulic fracturing hard data comparison-WVU pad and entire wells (77 wells)

\begin{tabular}{ccc}
\hline Parameters & All the Pads & WVU-Pad \\
\hline Number of stages & $8-10$ & $8-10$ \\
Number of cluster/stage & 3 & 3 \\
Stimulated lateral length(ft.) & $2500-3500$ & $2573-3102$ \\
Injected Proppant (lb) & $4,000,000-6,000,000$ & $3,794,742-5,099,860$ \\
Total slurry volume (bbl) & $120,000-150,000$ & $113,568-144,722.8$ \\
Average injection rate & $70-85$ & $55-81.6$ \\
Average injection pressure & $6000-8000$ & $3200-4654$ \\
\hline Initial shut in pressure & 3750 to 4250 & 5263 \\
\hline
\end{tabular}


Figure 3-15, shows the example of the stress distribution and created width profile and fracture conductivity distribution at the end of hydraulic fracturing treatment for one of the stages in one of WVU pad's horizontal laterals. Fracture propagates primarily only in the lower stress portion of the rock (Lower Marcellus). High stress barriers exist above and below the fracture, matching lithology changes and limit fracture growth vertically.

As shown in this figure, while the wetted length goes to $2300 \mathrm{ft}$., the propped half-length after closure does not exceed 500-550 ft. and the maximum conductivity in propped region is between 160 to $300 \mathrm{md}-$ $\mathrm{ft}$, which is too high. The calculated fracture properties (e.g. half-length, and conductivity) should be modified during the history matching process to match actual field measurement rates.

It should be noted that since the fracture propagation pressure is simply the Initial Shut-In-Pressure (ISIP) plus the extra pressure related to frictional effects from the injection, changes in the ISIP serve as a proxy for changes in $\mathrm{S}_{\mathrm{hmin}}$. Therefore, this parameter is used instead of minimum horizontal stress distribution for each cluster during the hydraulic fracture modeling, which represents the initial state of stress in the reservoir.

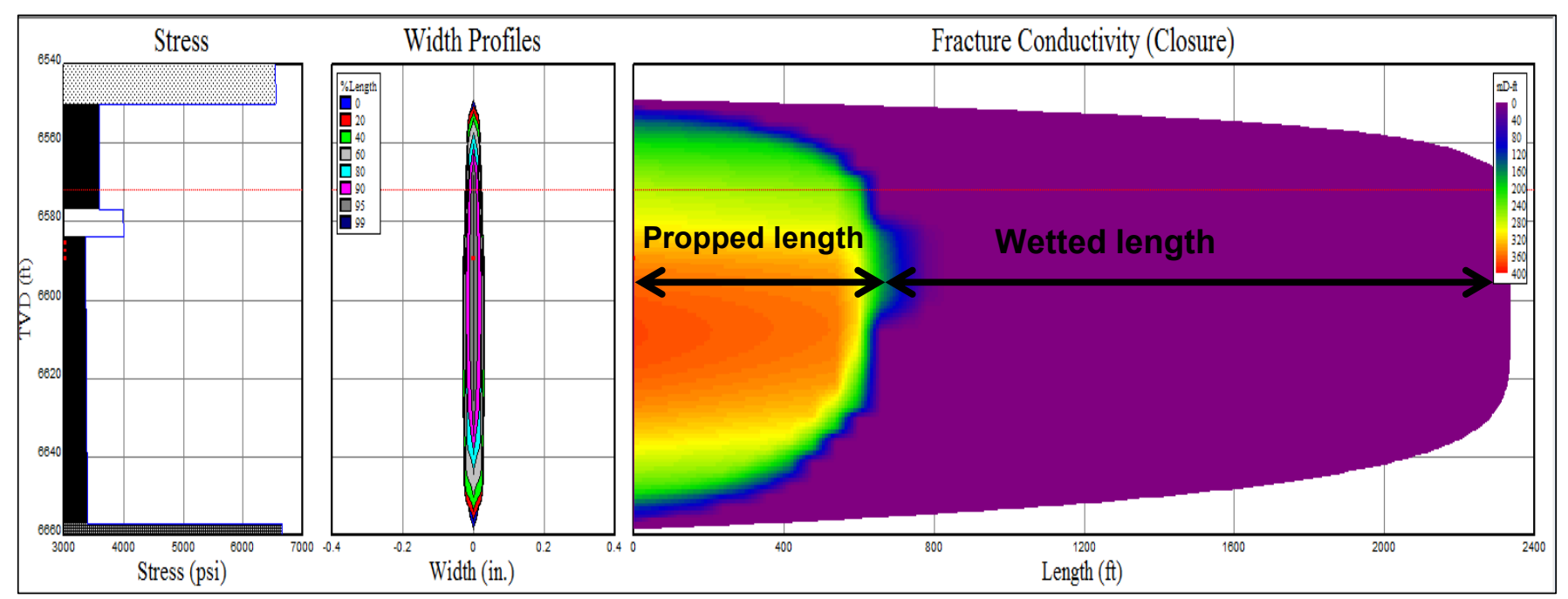

Figure 3-15.2D representation of generated hydraulic fracture with stress and width profile

As was mentioned before, due to computational limitation for performing numerical simulation, one of the pads with six horizontal wells with 169 clusters of hydraulic fracture is selected for history matching and the rest of the studies in this dissertation.

Figure 3-16 shows the entire study area with 77 horizontal wells and the location and configuration of laterals in WVU pad. 


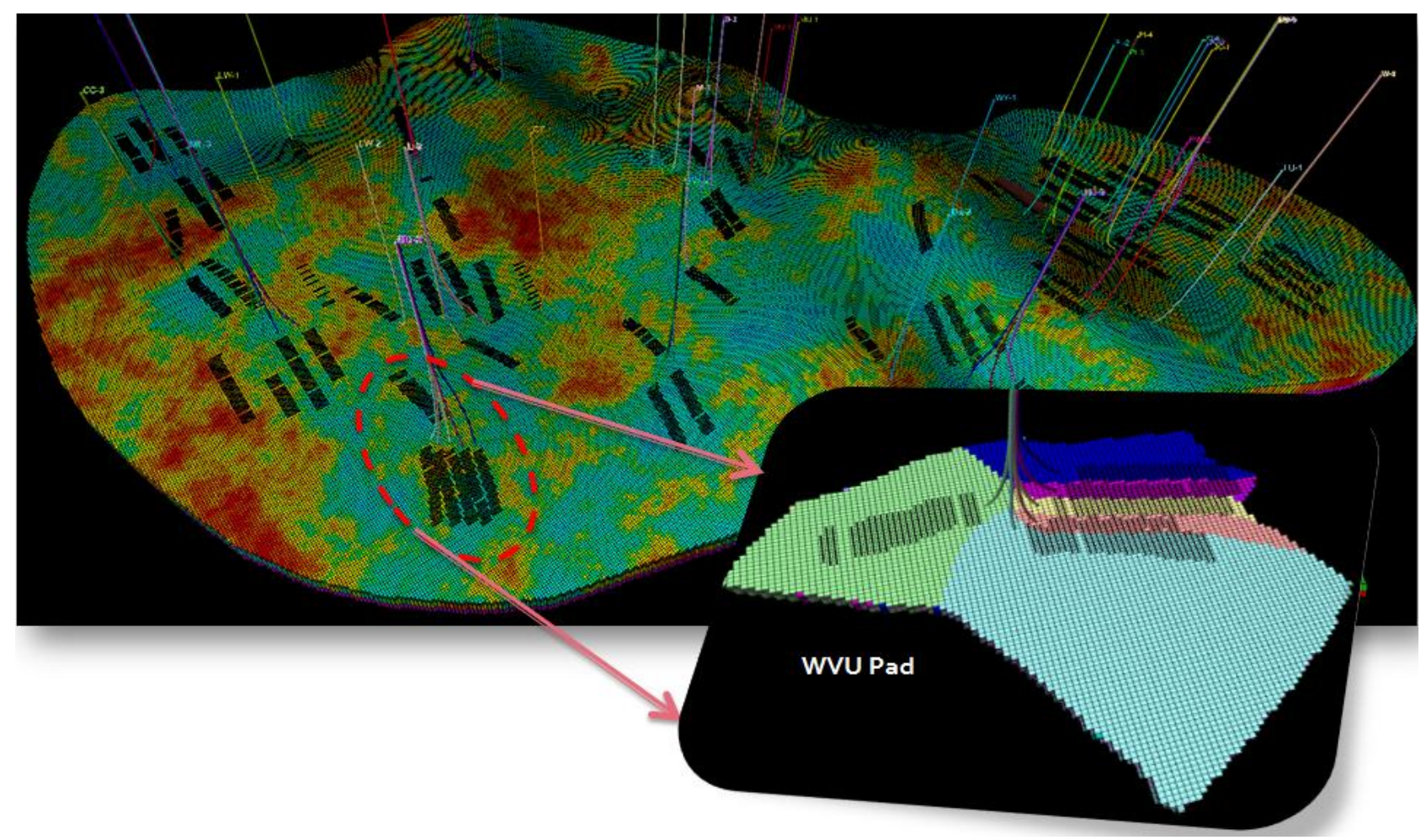

Figure 3-16-Local grid refinement for all the wells and the location and configuration of WVU pad

As can be seen from this figure, logarithmic local grid refinement (LGR) is performed and each host cell is divided into seven grid blocks laterally and three vertically. The finest grid has $1 \mathrm{ft}$ width and represents the hydraulic fracture in the reservoir simulation model, which possesses the hydraulic fracture characteristics that are calculated in the previous step. The schematics of host cells (left), LGRs and the distribution of fracture conductivity (right) for all the clusters in WVU- Pad are shown in Figure 3-17.

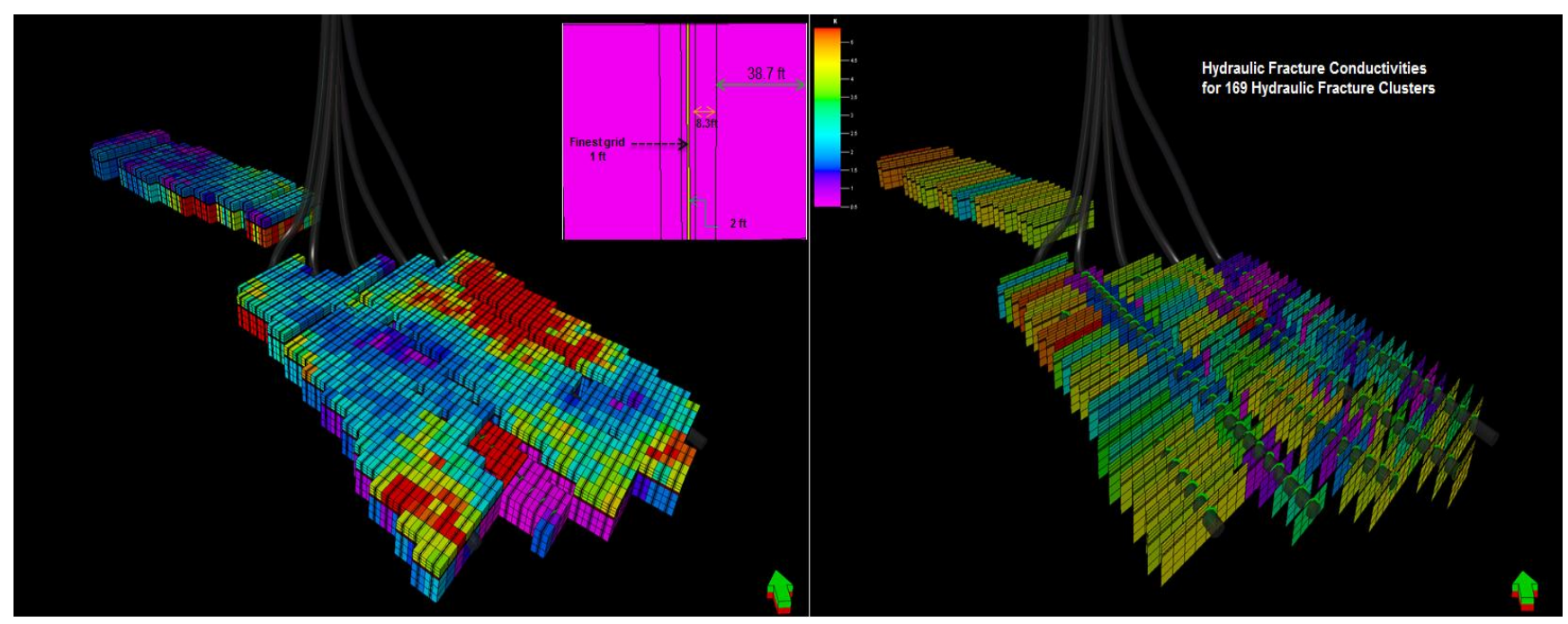

Figure 3-17.3D LGR representation and distribution of hydraulic fractures conductivity 


\subsection{History Matching and Forecasting Production}

The last step in the development of shale gas simulation model is to adjust the geological model and its parameters such that the simulation model is able to reproduce the gas rate and bottom-hole pressure histories reasonably. This history matching is an inverse problem, meaning that there is no unique solution and new simulation model can be different from the base geological model.

Generally, in modeling and simulation, two time-consuming tasks are data gathering and history matching. Shortcuts in gathering data often increase the time needed for history matching because either limited or bad data require additional trial-and-error iterations. Moreover, in case of EHF modeling long simulation time is a serious issue, which makes the history matching process even more complicated.

As was mentioned earlier, almost all the numerical simulation studies conducted on shale formations so far, (At the time of writing this dissertation) are single well models. The reason for that is not just limited to long, laborious model setup, and computationally expensive implementation of it, the interferences between the hydraulic fractures in a Pad with multilateral makes the history matching for those wells very tough. Any changes in target well's matrix and especially natural and hydraulic fracture characteristics have positive or negative impact on the history matching results for the offset laterals.

Dual porosity, compositional simulation model with around 200,000 grid blocks with three simulation layers in non-refined regions and nine simulation layers in the refined regions, and shale related keywords is set up to history match almost 2.5 years of daily gas production for six horizontal laterals in WVU pad. Methane and Carbon dioxide are two main compositions with 99.9 and 0.1 percent relatively. Figure 3-18 shows the entire WVU pad configuration and corresponding six horizontal laterals.

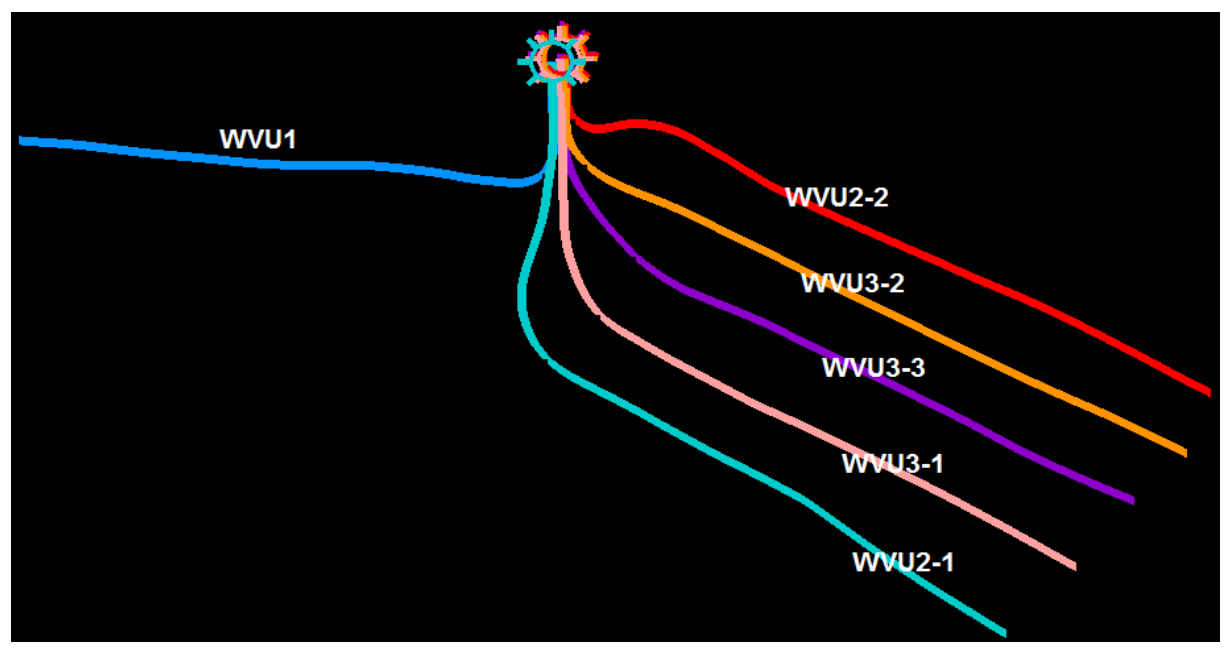

Figure 3-18. WVU Pad configuration and corresponding horizontal laterals 
Figure 3-19 illustrates the measured daily gas production rate (top) and the calculated bottom-hole pressure (bottom) from measured wellhead pressure for all six horizontal laterals in WVU pad.

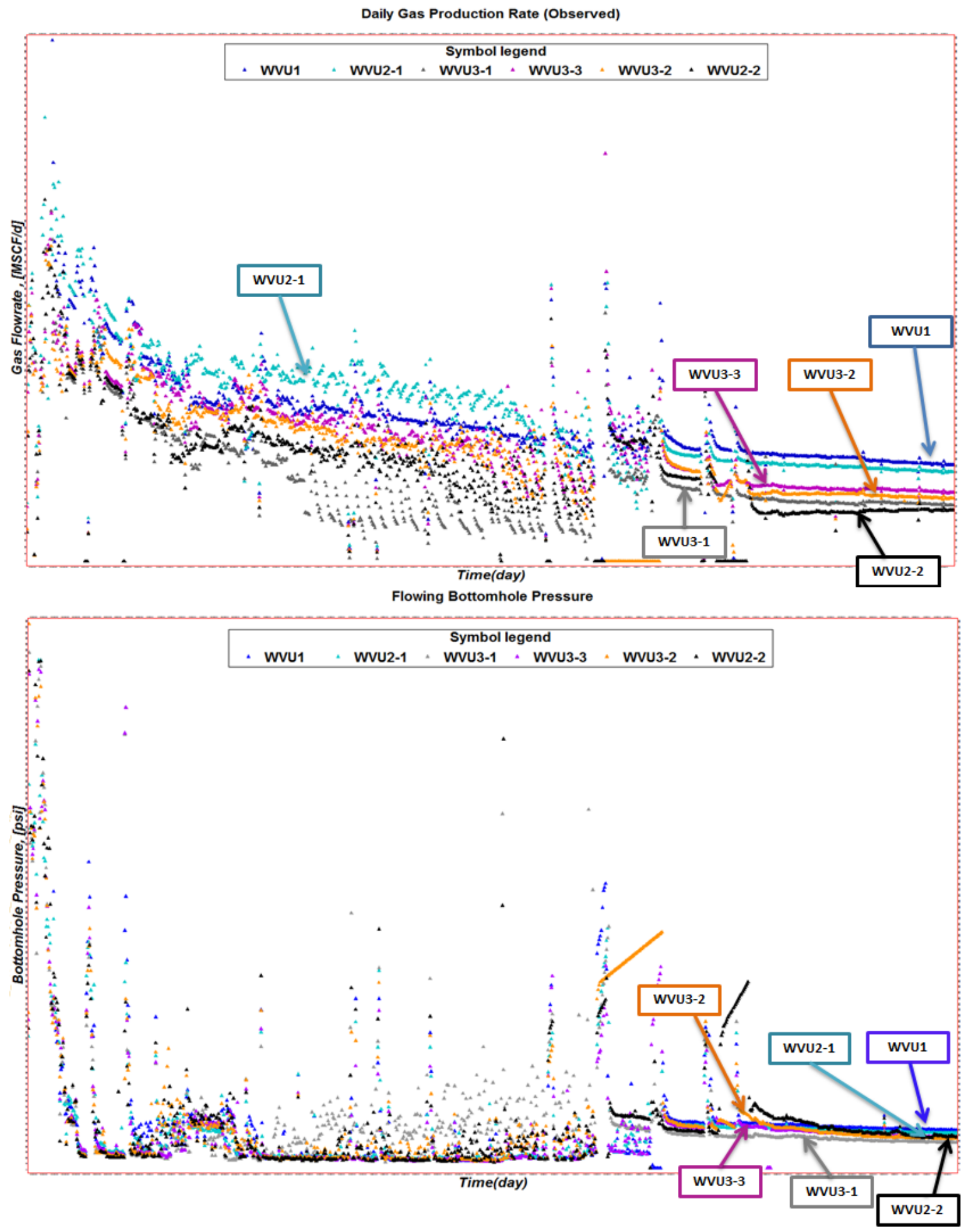

Figure 3-19. Measured daily gas production and bottomhole pressure for all the wells in WVU Pad (Top to bottom relatively) 
In order to perform the history matching and forecast the production for all the wells in WVU pad, a multidisciplinary approach to build a base geological model, which integrates aspects related to reservoir characteristics, Geomechanics, completion and stimulation, is utilized and was explained in previous sections.

A detailed workflow showing the interaction among all disciplines involved in the base model development, history matching and forecasting is highlighted and summarized in Figure 3-20. Some of key reservoir characteristics (e.g. matrix porosity, natural fracture porosity, natural fracture permeability, rock fraction etc.), hydraulic fracture properties (e.g. HF length and conductivity) and some other completion related parameters such as skin factor are tuned manually to achieve a satisfactory history match for all individual laterals and the entire pad. As was mentioned earlier, apart from long simulation time, the interference between WVU2-1, WVU2-2, WVU3-1, WVU3-2 and WVU3-3 makes the process very tricky.

The key in achieving a good history matching result is having in depth understanding of physics that is involved in shale production and is implemented in reservoir simulator, which is ECLIPSE 2012.1 (Schlumberger proprietary reservoir simulation software) in this study. For performing the historymatching, ECLIPSE is run in flowing bottomhole pressure control mode. It means that the bottomhole pressure is constrained in the simulation and the measured daily gas rate production is set as a target to be matched.

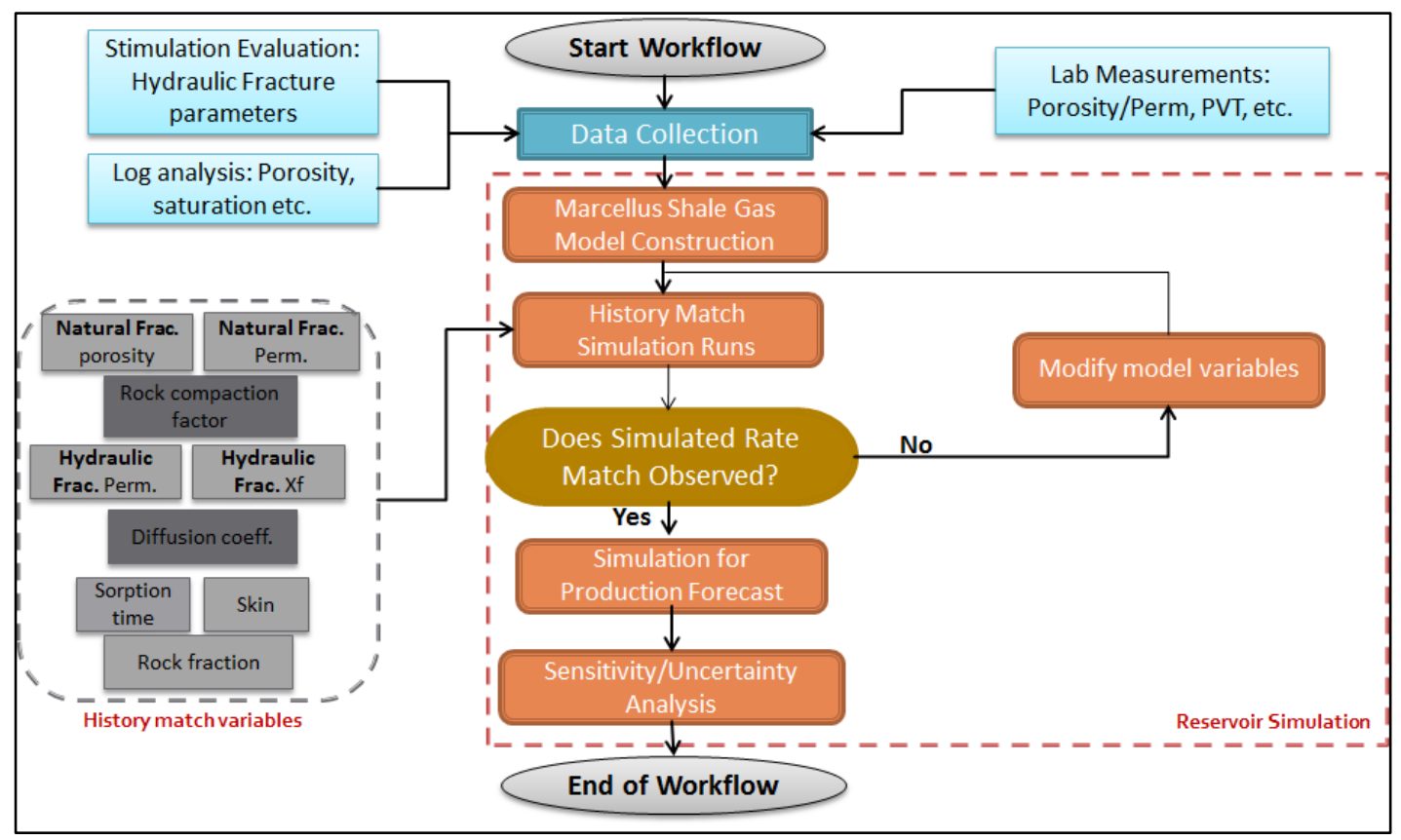

Figure 3-20.An integrated workflow flowchart illustrating all steps from base model development to history matching 


\subsubsection{Key History Matching Variables and Lesson Learned}

In this part, some of the key history matching parameters that play significant role in achieving a good enough history matching results and lesson learnt during this process are discussed.

As highlighted in Figure 3-20, natural fracture, hydraulic fracture and some of matrix properties and completion related parameters such as skin factor are among the most important variables for history matching.

Hydraulic fracture Conductivity (HFC) -Is one of the important history matching parameters. Increasing this value causes the simulated gas production rate profile to increase by a positive offset with little change in its slope. The relationship between the increase in hydraulic fracture conductivity and the offset shift in the gas rate is not linear. The sensitivity in the increase in gas rate offset by an increase in hydraulic fracture conductivity depends on the values of the other variables such as hydraulic fracture half-length.

Hydraulic fracture half-length $\left(\boldsymbol{X}_{f}\right)$ - Increasing the half-length causes the gradient of the simulated gas rate to decrease (get less steep). In addition, there is an increase in the simulated gas offset. This increase in offset is generally smaller than the increase observed hydraulic fracture conductivity especially at early times.

Natural fracture porosity $\left(\boldsymbol{\phi}_{f}\right)$-Increasing the value of the fracture porosity results in more gas production at the early stage (beginning) of production and is a key parameter to control IP (Initial Potential) and capturing sharp decline at the beginning of production.

Sigma- Accounts for the matrix/fracture interface area per unit matrix volume. Sigma values lower than 0.08 yields the spacing greater than $10 \mathrm{ft}$. Sensitivity studies show that any increase in Sigma value beyond 0.08 has minimal impact on the simulated gas rate.

Matrix porosity $\left(\boldsymbol{\phi}_{m}\right)$-Which refers to gas-filled porosity in pores does not have a significant impact on the production at the beginning, but generally any increase, elevates the production profile by positive offset.

Figure 3-21, Figure 3-22 and Figure 3-23 show the history matching results for six horizontal laterals in WVU Pad. The location of each lateral in the pad is illustrated in each plot as well. In all plots, dots refer to measured daily gas production (Mscf/d) and the solid lines show Eclipse simulation result. 
WVU1-History Matching Result

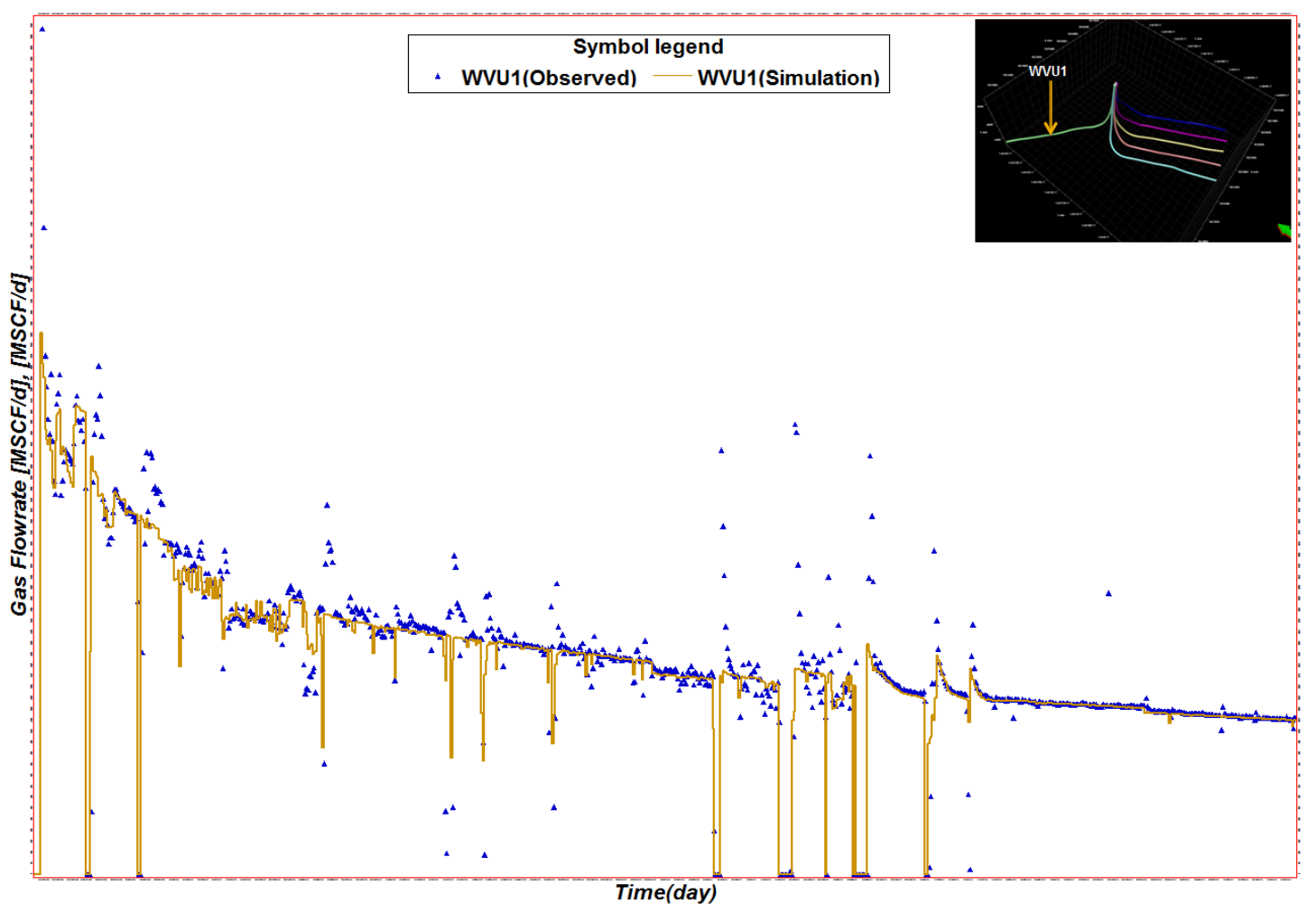

WVU2-1-History Matching Result

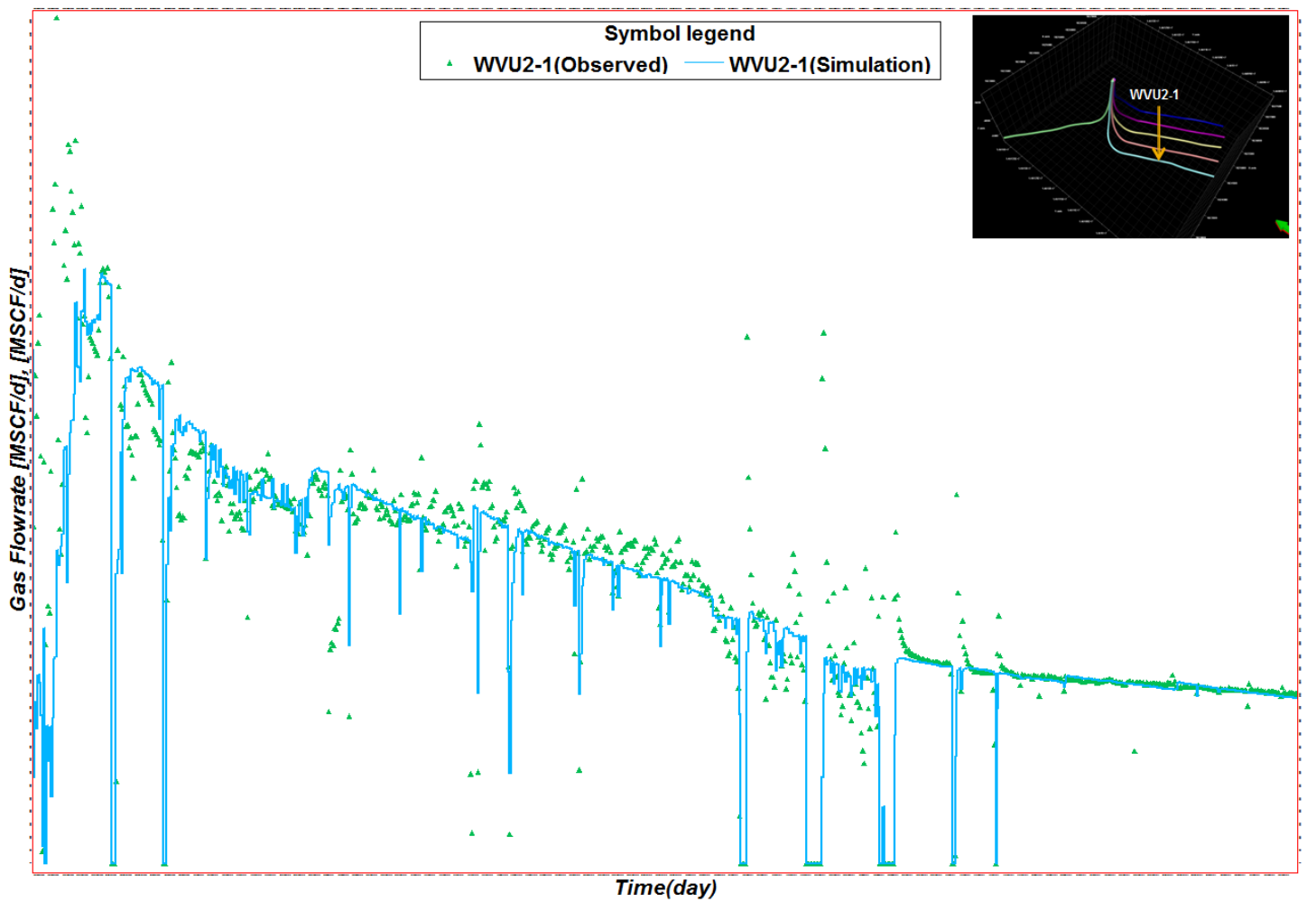

Figure 3-21.Comparison of actual daily gas rate data points with the simulation results from Eclipse for WVU1 and WVU2-1 

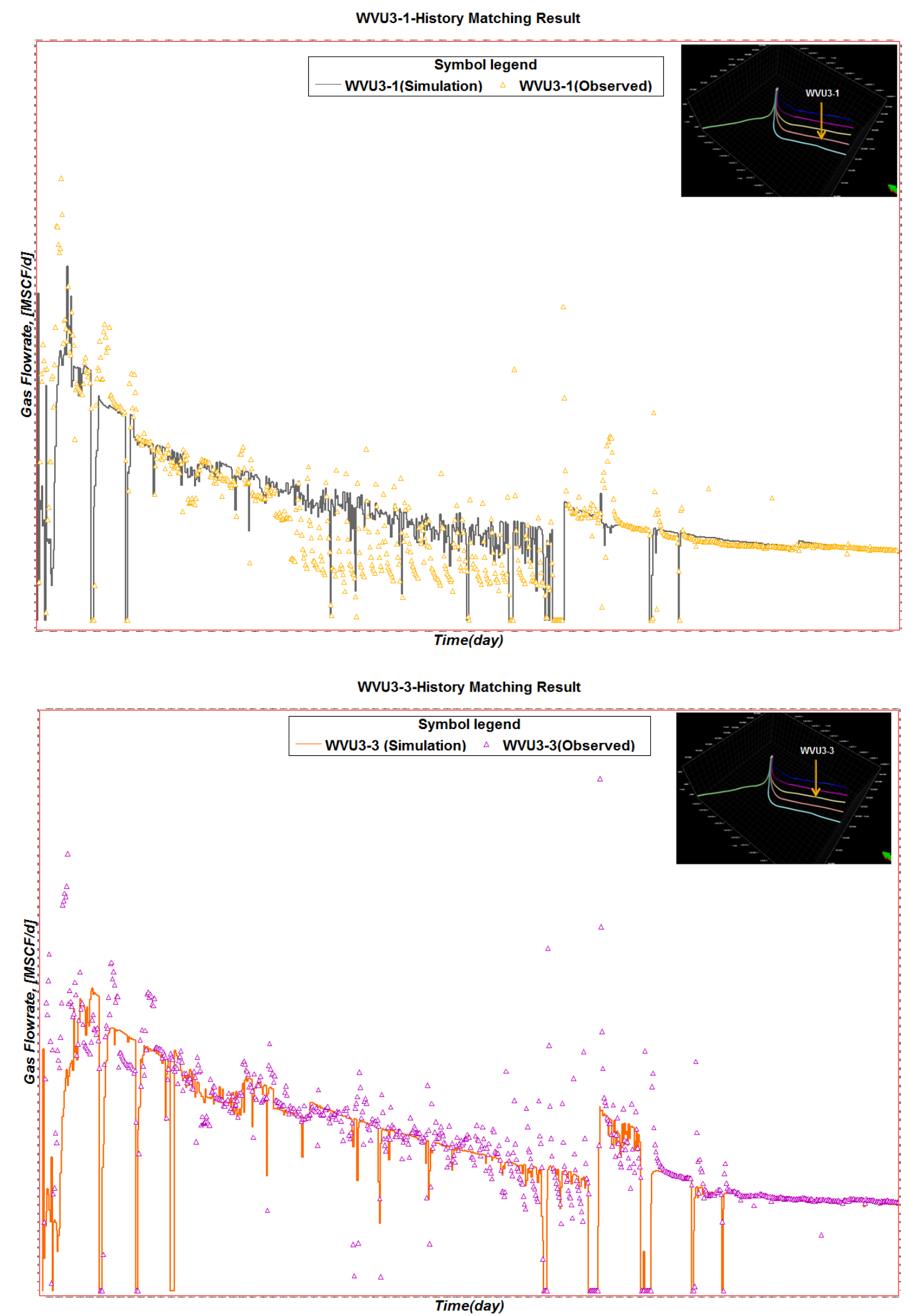

Figure 3-22.Comparison of actual daily gas rate data points with the simulation results from Eclipse for WVU3-1 and WVU3-3 

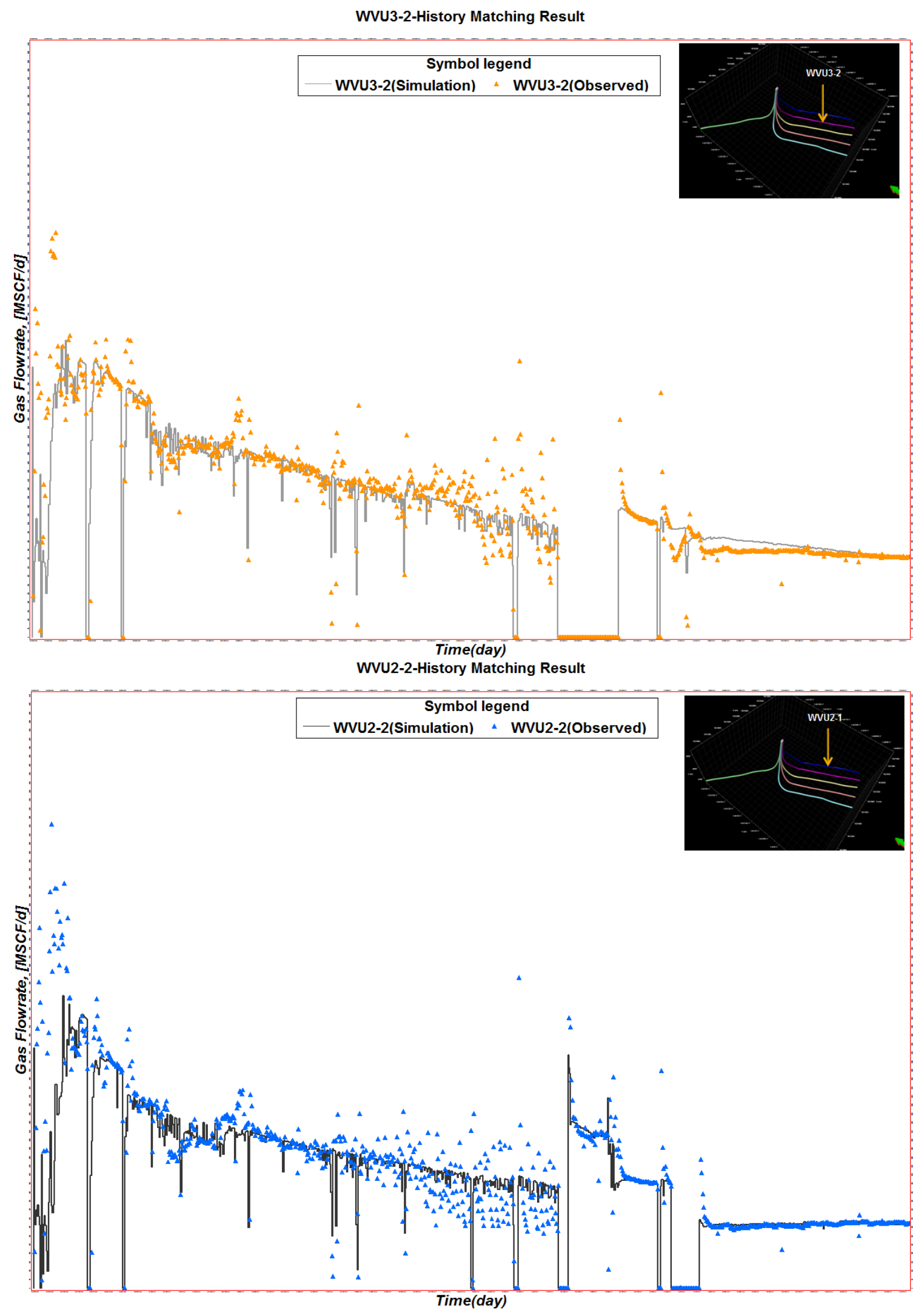

Figure 3-23.Comparison of actual daily gas rate data points with the simulation results from Eclipse for WVU3-2 and WVU2-2 


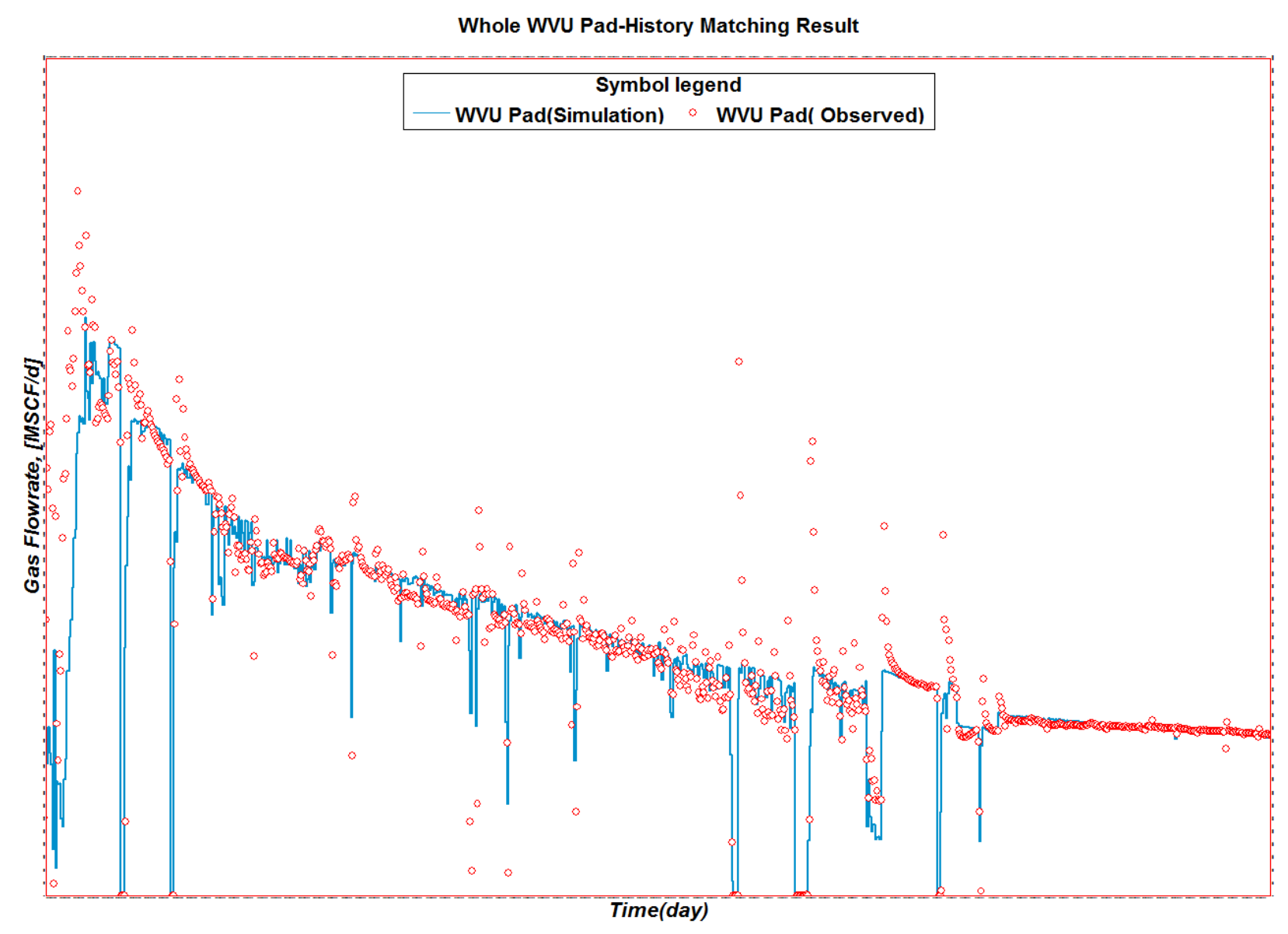

Figure 3-24.Comparison of actual daily gas rate data points with the simulation results from Eclipse for entire WVU pad

As can be seen from these graphs, acceptable history matching results are achieved. In addition, the history matching result for entire WVU pad is illustrated in Figure 3-24, which shows a good match between the measured and simulated daily gas production for entire pad.

As can be seen from the production profile (Figure 3-25) for almost all the laterals after a period of production the wells are shut and then -opened with higher production rate. This behavior might be due to the fact that gas wells can commonly experience a loading up of water in the wellbore after a period of time. This water can exert a backpressure on the formation and act as a "choke" on the production. Therefore, the operators may stop production to clean the wellbore and remove the water, then install production tubing into the well to help lift the liquids and optimize the gas production. Immediately after the tubing is installed, it is common to see a change in well's production profile. The flowing bottomhole pressure is also seen to increase at this point (due to less liquid loading in the wellbore). In order to capture this behavior during the history matching, "WPIMULT" keyword is introduced to the simulation. 

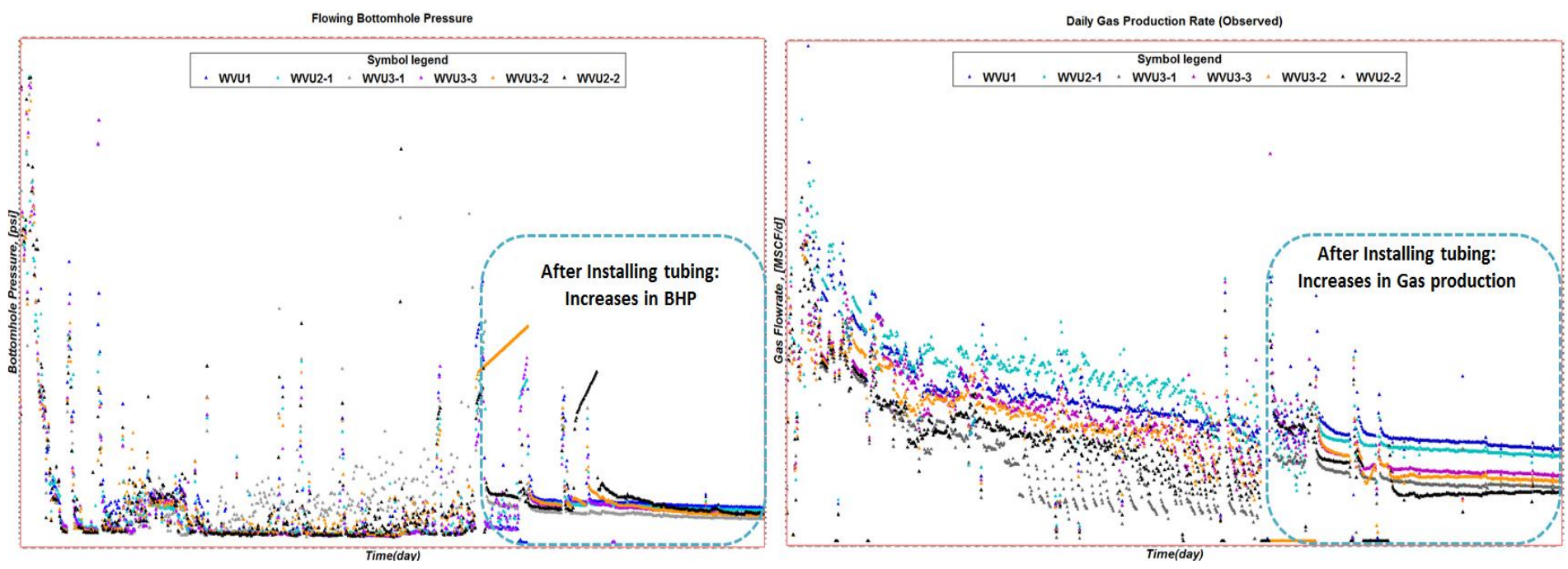

Figure 3-25. Gas production rate and bottomhole pressure changes for most of the wells after installing tubing

The WPIMULT keyword multiplies the connection transmissibility factor of the perforations open to flow. A value greater than 1.0 increases connection transmissibility, and a value less than 1.0 decreases it. For example, WPIMULT value of 1.3 increases the connection transmissibility by $30 \%$.

History matching values for some of the parameters that are either discrete or do not have the distribution map are summarized in Table 3-4 for each horizontal laterals. For the rest of properties in the history matched model such as hydraulic fracture conductivity, natural fracture and matrix permeability, matrix porosity and net to gross ratio for upper and lower Marcellus, the distributions are provided in Figure 3-27 through Figure 3-30. Red color shows the maximum and purple one represents the minimum value for each parameter.

Table 3-4. History matching parameters and corresponding values for each well

\begin{tabular}{|c|c|c|c|c|c|c|c|c|c|c|c|c|c|}
\hline $\begin{array}{l}\text { Well } \\
\text { Name }\end{array}$ & WPIMULT & Skin & $\begin{array}{c}\text { HF } \\
\text { length(ft) }\end{array}$ & $\begin{array}{l}\text { No. of } \\
\text { clusters }\end{array}$ & $\boldsymbol{\Phi}_{\mathrm{f}}$ & $\begin{array}{c}\text { Diff.Coeff. } \\
\left(\mathrm{Ft}^{2} / \mathrm{day}\right)\end{array}$ & $\begin{array}{c}\mathrm{VL}_{\mathrm{CH} 4} \\
\text { (Sef/ton) }\end{array}$ & $\begin{array}{c}\mathrm{PL}_{\mathrm{CH} 4} \\
\text { (psi) }\end{array}$ & $\begin{array}{c}\mathrm{VL}_{\mathrm{CO} 2} \\
\text { (Scf/ton) }\end{array}$ & $\begin{array}{c}\mathbf{P L}_{\mathrm{CO} 2} \\
\text { (psi) }\end{array}$ & $\begin{array}{l}\text { Kv/Kh } \\
\text { Matrix }\end{array}$ & $\begin{array}{c}\text { Kv/Kh } \\
\text { Fracture }\end{array}$ & Sigma \\
\hline WVU1 & 1.05 & 3 & $\begin{array}{l}700- \\
1100\end{array}$ & 24 & 0.035 & 0.7 & 73 & 726 & 120 & 400 & 0.1 & 0.5 & 0.06 \\
\hline WVU2-1 & 1.28 & 4.2 & $600-800$ & 29 & 0.031 & 0.7 & 73 & 726 & 120 & 400 & 0.1 & 0.5 & 0.055 \\
\hline WVU3-1 & 1.2 & 4.5 & $200-500$ & 30 & 0.023 & 0.7 & 73 & 726 & 120 & 400 & 0.1 & 0.5 & 0.02 \\
\hline WVU3-3 & 1.32 & 5.1 & $500-700$ & 30 & 0.023 & 0.7 & 73 & 726 & 120 & 400 & 0.1 & 0.5 & 0.032 \\
\hline WVU3-2 & 1.12 & 4.8 & $300-500$ & 29 & 0.023 & 0.7 & 73 & 726 & 120 & 400 & 0.1 & 0.5 & 0.03 \\
\hline WVU2-2 & 1.1 & 5.3 & $400-500$ & 27 & 0.026 & 0.7 & 73 & 726 & 120 & 400 & 0.1 & 0.5 & 0.015 \\
\hline
\end{tabular}




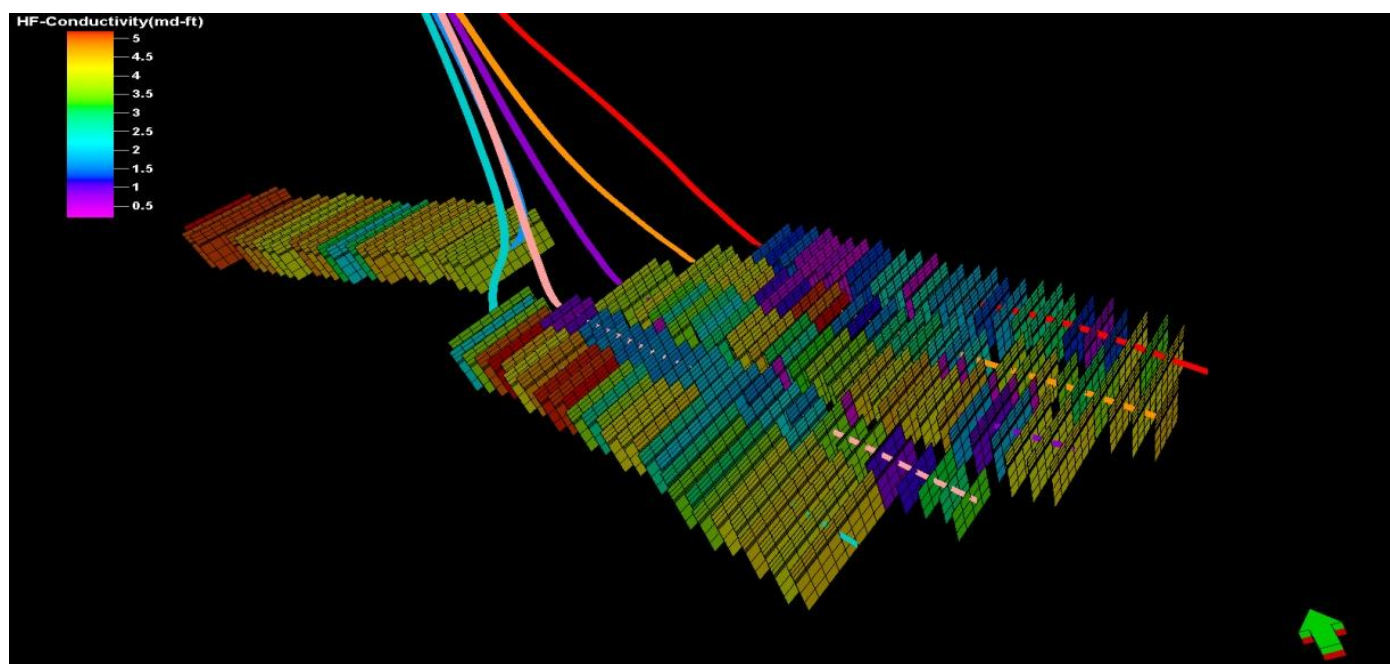

Figure 3-26. 3D representation of hydraulic fracture conductivity for 169 clusters (Min. value=0.5 and Max.=5.4 md-ft)

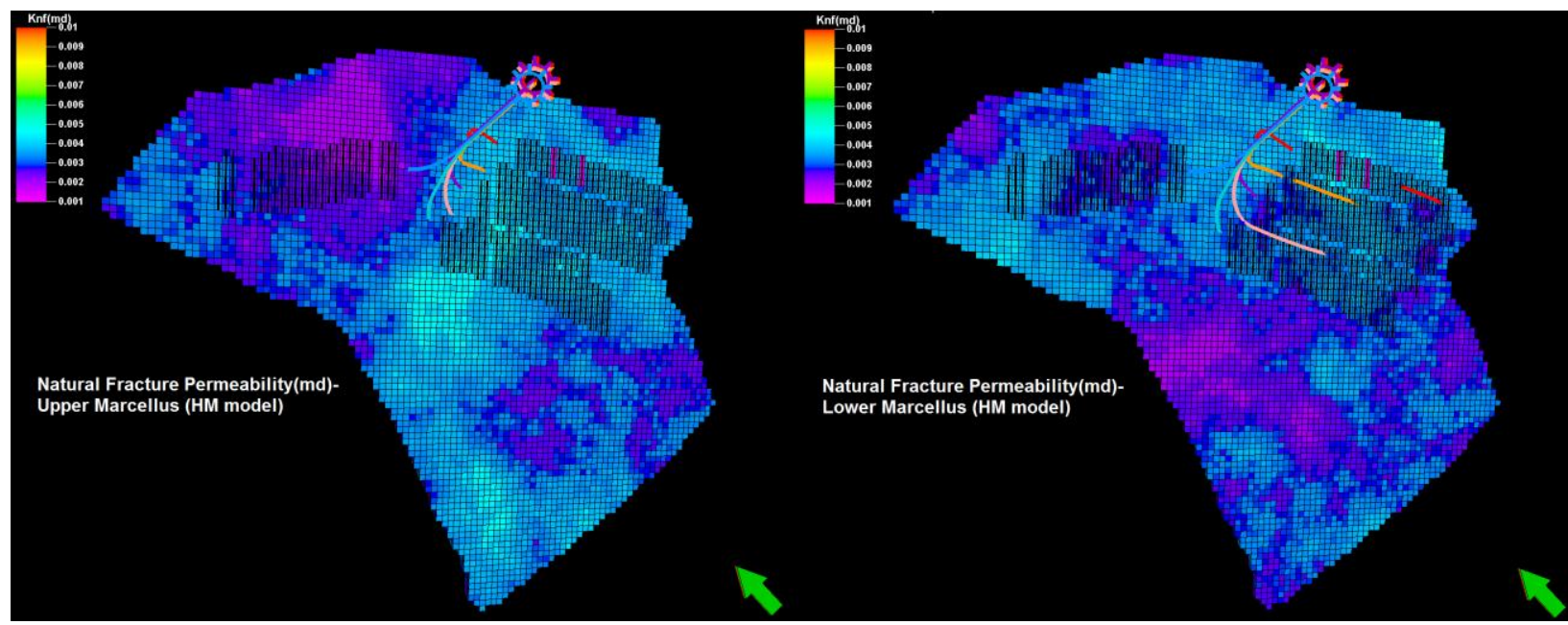

Figure 3-27.Natural fracture permeability distribution in UM and LM from left to right (Min. value=0.001 and Max.=0.01 $\mathrm{md}$ )

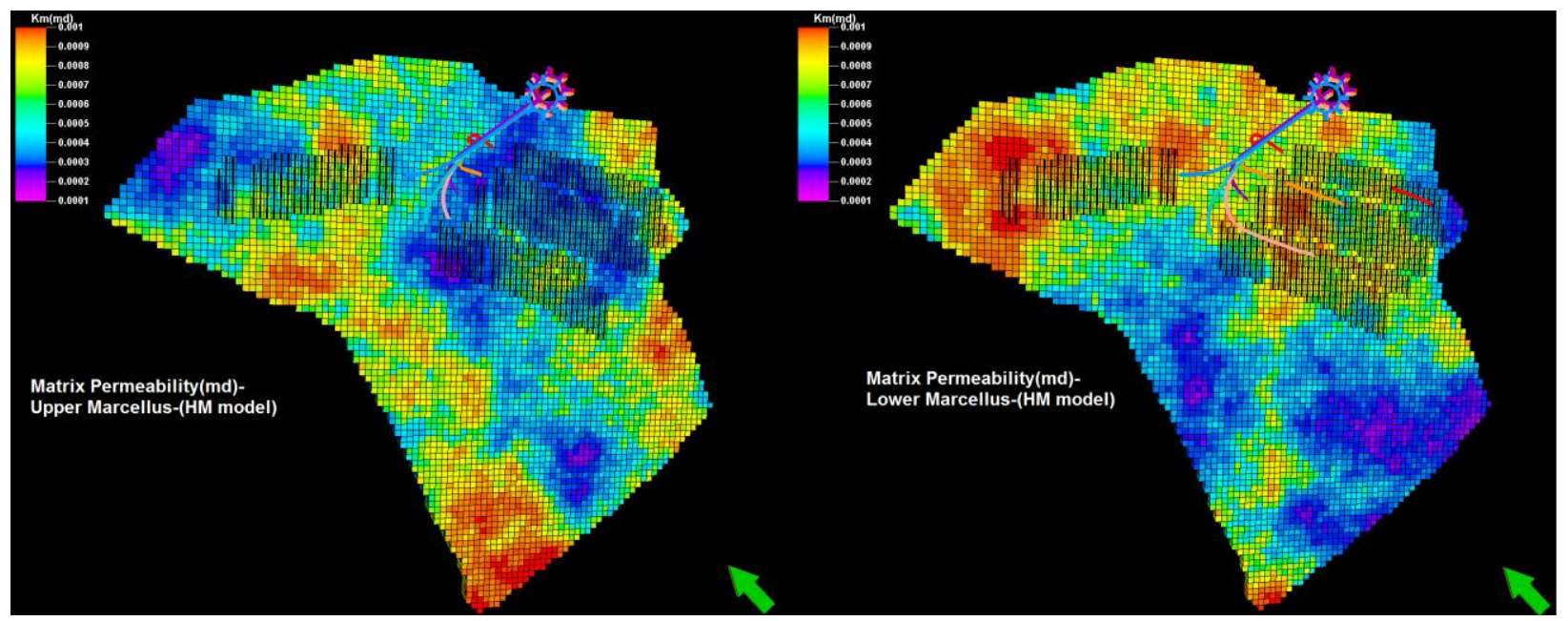

Figure 3-28. Matrix permeability distribution in UM and LM from left to right (Min. value $=0.0001$ and Max. $=0.001 \mathrm{md}$ ) 


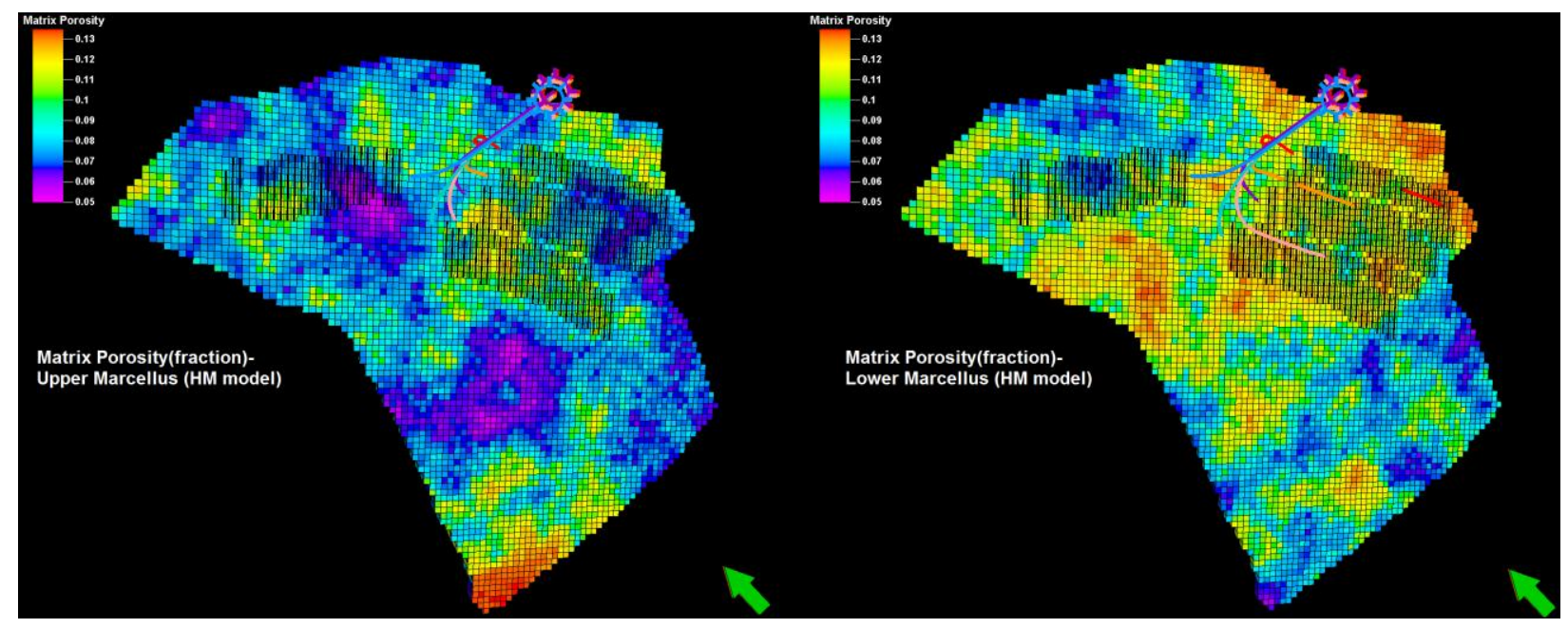

Figure 3-29. Matrix porosity distribution in UM and LM from left to right (Min. value=0.05 and Max. =0.135 $\mathrm{md}$ )

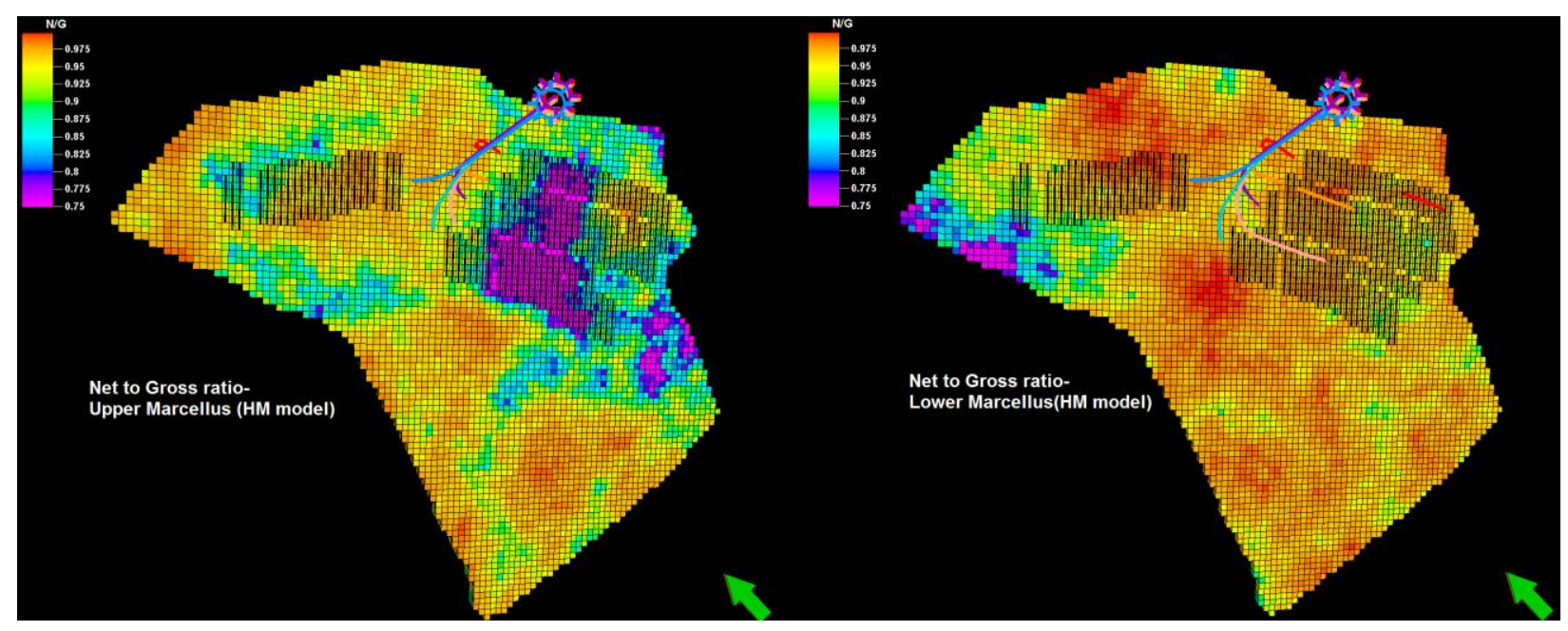

Figure 3-30. NTG distribution in UM and LM from left to right (Min. value=0.75 and Max. $=0.98 \mathrm{md}$ )

After completing the history matching process, the simulation is continued for 90 years to forecast the production for each horizontal lateral in WVU pad by taking into account the economic gas production limit of $50 \mathrm{Mscf} /$ day. Figure 3-31 shows the production profile for all laterals until reach to the economic limit.

As can be seen from this plot, WVU1 and WVU2-1 are still producing above the set economic limit after 90 years, while WVU2-2, WVU3-1, WVU3-2, and WVU3-3 are reached to their economic limits after $39,56,77$ and 28 years respectively.

The pressure distributions for entire WVU pad after 6 months, 1 year, 3, 5, 10, 20, 30, 60 and 90 years of production are illustrated in Figure 3-32. In this figure, the pressure is changing from 3890 psi, which is the initial reservoir pressure to the minimum value of 370 psi after 90 years of production. It should be 
noted that in some part of the reservoir the pressure is still around 2800 psi after 90 years of production due to very low matrix permeability and very low gas diffusion rate.

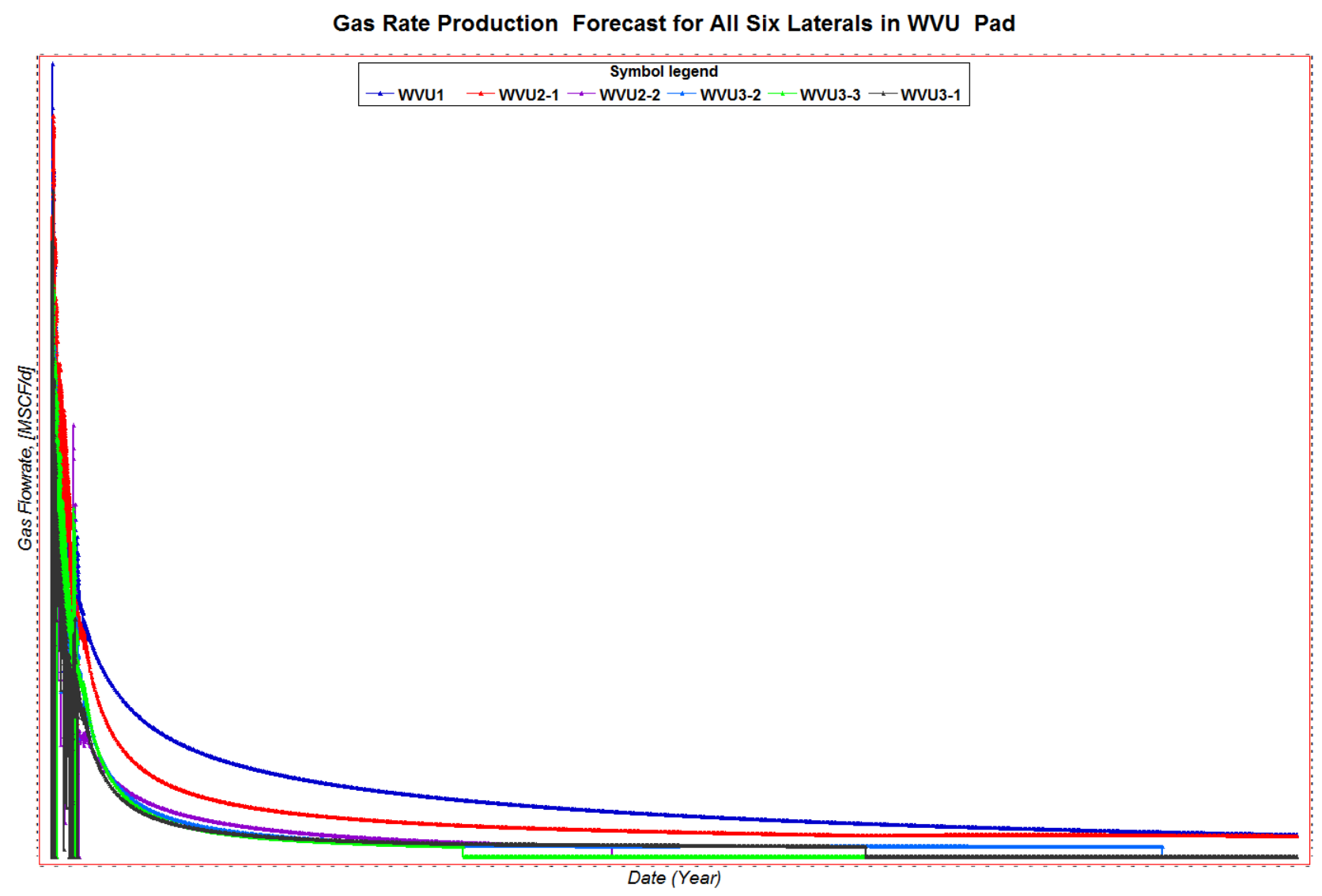

Figure 3-31. Forecasting production behavior foal I WBVU pad's laterals

\subsection{Summary and Conclusions}

In this chapter an integrated workflow, which demonstrates a quantitative platform for shale gas production modeling and simulation through capturing the essential characteristics of the shale gas reservoir is proposed.

In this workflow, dual porosity, compositional simulation with explicit representation of hydraulic fracture is used to perform history matching and production forecast. A representative pad in terms of reservoir and hydraulic fracture characteristics in the large Marcellus shale study area in SW PA is selected to perform the simulation. History matching process is successfully completed for all the laterals (six horizontal laterals in WVU Pad) and the production is forecasted for 90 years. 
Nearly all the numerical simulation studies conducted on shale formations so far, are single well models because of long and laborious model setup and computationally expensive implementation of it, and also the interferences between the hydraulic fractures in a Pad with multilaterals makes the history matching job very difficult. Therefore, there is a need to develop a technique to make this process easier and performing uncertainty analysis more efficient.

The developed history matched model is the basis for all future analysis in this dissertation such as developing next-generation Data-driven shale proxy model and $\mathrm{CO}_{2}$-Enhanced gas recovery and storage.

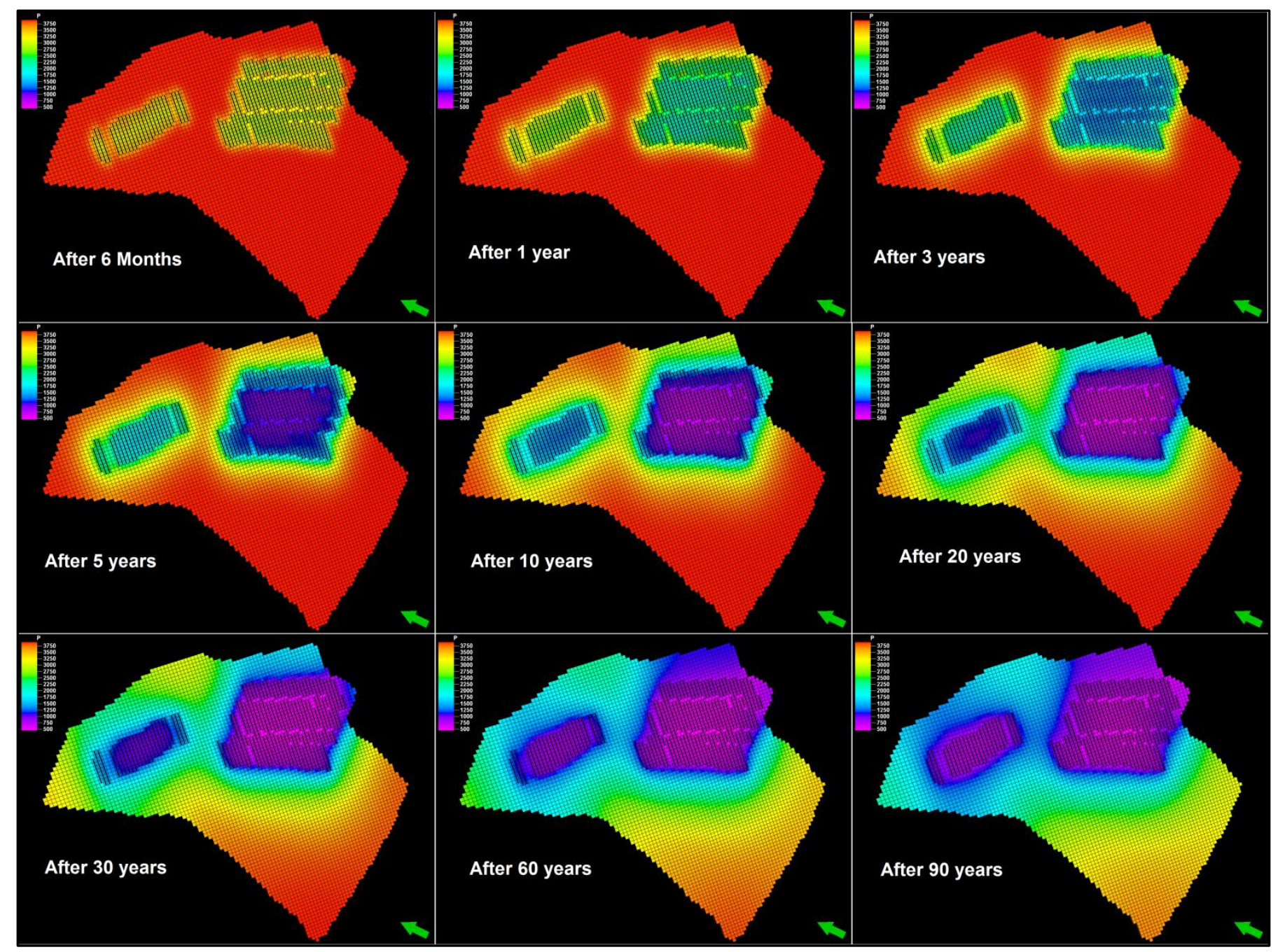

Figure 3-32. Pressure distribution in matrix after 6 month, 1 year, 3,5,10,20,30,60 and 90 years of production 


\section{CHAPTER 4 -DATA DRIVEN MODEL, A NEXT-GENERATION SHALE PROXY AT HYDRAULIC FRACTURE CLUSTER LEVEL-PRODUCTION PHASE}

\subsection{Summary}

As was mentioned previously, EHF modeling attempts to honor the impact of hydraulic fracturing at the cluster/stage level by incorporating hydraulic fracture characteristics in the simulation model. Nevertheless, model setup for the EHF technique is long and laborious and its implementation is computationally expensive, such that it becomes impractical to model beyond a single pad.

In this chapter, a Next-generation shale proxy model (Data-driven proxy model) based on pattern recognition capabilities of Artificial Intelligence is introduced. A detailed procedure for development of this data-driven proxy model, which is capable to reproduce the simulation output(169 production rate profiles for 169 clusters of hydraulic fracture) with acceptable accuracy, is explained .The proxy model is validated using blind simulation run that is not used during the neural network training and validation.

\subsection{Data-driven Shale Proxy Model}

Date driven shale proxy model, which is based on the pattern recognition capabilities of artificial intelligence and data mining, has several advantages over traditional proxy models such as response surfaces or reduced ordered models. These advantages include:

- No need to approximate the existing simulation model to develop an AI-based proxy model

- The number of simulation runs required for the development of an AI-based proxy model is at least an order of magnitude less than traditional proxy models, and

- Beyond representing the pressure and production profiles at each well individually, AI-based shale proxy model can replicate, with high accuracy, the pressure and molar density changes at each grid block.

During the development process, numerical reservoir simulation model is used to teach the Data-driven proxy model the principles of fluid flow through shale porous media and the complexities of the heterogeneous reservoir represented by the geological model and its impact on the fluid production and pressure changes in the reservoir.

Unlike other proxy models that require hundreds of simulation runs for their development, the proposed proxy model, requires only a small number of simulation runs to be used for representative spatio- 
temporal database generation. This database is uniquely built for each problem by designing and running several reservoir simulation cases/realizations, to teach the neural network the impact of changing all parameters that are involved in shale gas modeling (e.g. matrix and natural fracture properties, hydraulic fracture characteristics, sorption type and flow regimes, operational constraint). In other words, all that you want the neural network to learn and to be able to accurately reproduce the output/s must be included in this database.

Necessity of Date-driven shale proxy model has to do with the fact that massive potentials of the existing numerical reservoir simulation models go unrealized because it takes a long time to make a single run. Numerical models that are built to simulate complex reservoirs with multi-million grid blocks require considerable run-time even on a cluster of parallel CPUs.

Exhaustive and comprehensive evaluation of the solution space for designing field development strategies as well as quantification of uncertainties associated with the static model are the type of analyses that require a large number of simulation runs in order to provide meaningful and usable results. When a numerical simulation model takes hours for a single run, performing such analyses become impractical and the engineers have to compromise by designing and running a much smaller number of runs in order to make decisions.

When the quantification of uncertainties associated with the geologic model is one of the objectives, Data- driven shale proxy model can prove to be a valuable asset. Since a single run of the full-field model (Single pad with multiple horizontal laterals in our study) takes several hours, history matching and uncertainty analysis become a painful and time-consuming process and almost impractical.

In order for the uncertainty analysis to be meaningful, the number of geologic realizations of the reservoir must be statistically significant. This is the reason behind the fact that most of the techniques used for quantification of uncertainties call for analysis of several (sometimes hundreds of) geological realization of the reservoir. As the number of independent parameters involved in a problem increases, so does the number of realizations needed for statistical significance. (Mohaghegh 2011, 2012)

\subsection{Methodology}

Inclusive spatio-temporal database generation is the starting point and the most important step toward building a Data-driven proxy model. The history-matched model with nine additionally defined realizations, are used to build an expert database, which is capable of teaching the multilayer feed- 
forward back-propagation neural networks, highly non-linear and complex shale system behavior and regenerate production profiles for all 169 clusters of hydraulic fractures for six horizontal laterals.

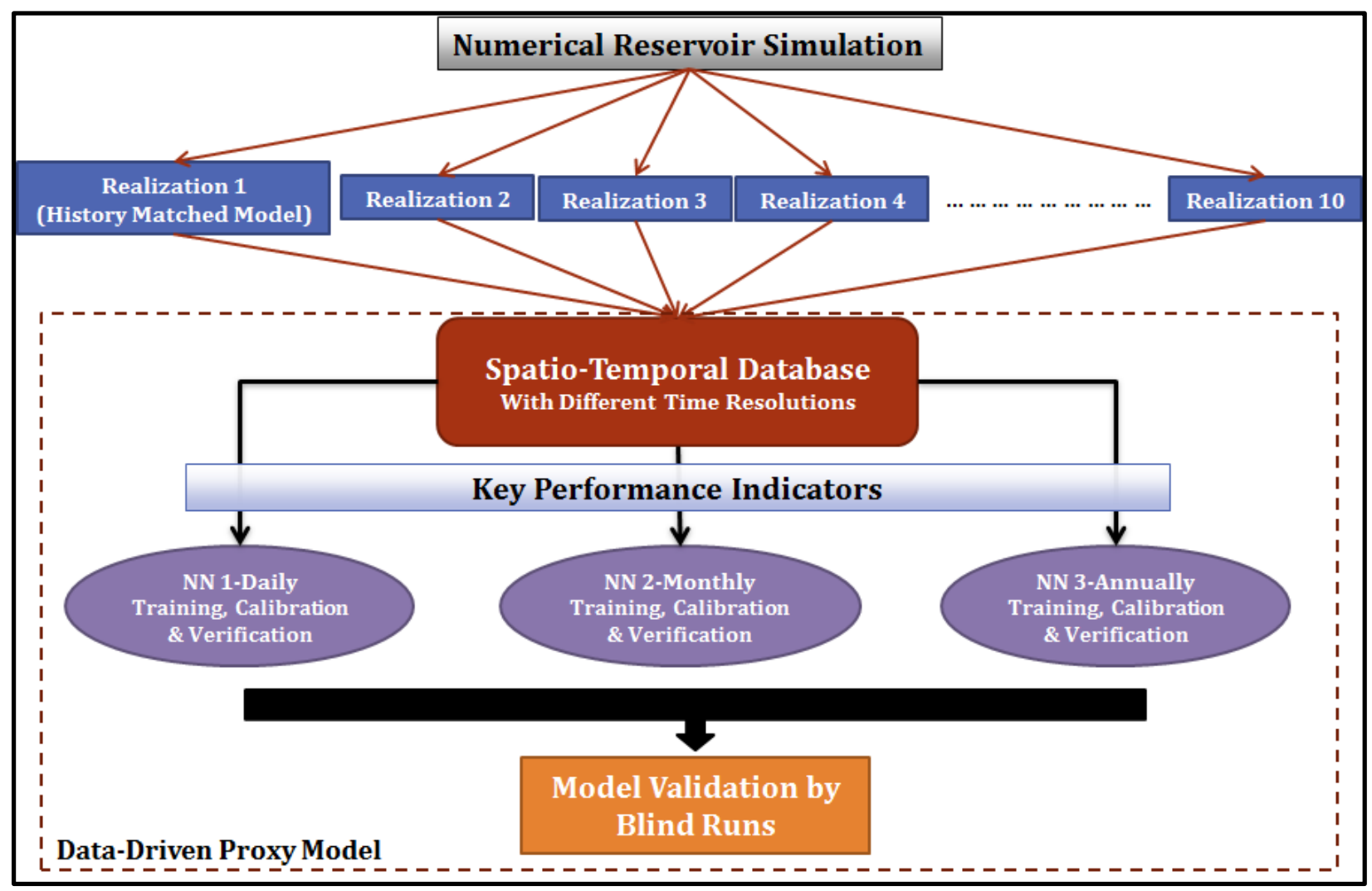

Figure 4-1. Data-driven Shale proxy model development workflow.

Data-driven proxy model generation workflow is completed by validating the neural networks (NNs) with a completely different (but in the uncertainty range) and blind simulation run that is not used during training, calibration and verification step. Figure 4-1 shows the detailed workflow for Data-driven shale proxy model development.

\subsection{Spatio-Temporal Database Generation}

As briefly mentioned before, the first and the most important step in the development of any data-centric reservoir model is preparing a representative spatio-temporal database, which forms the foundation of Data-driven shale proxy model.

The extent at which this database actually represents the fluid flow behavior of the reservoir that is being modeled, determines the potential degree of success in developing a successful Data-driven shale proxy model. 
This database includes the input-output pairs of data that is used for the training process. The inputs are static data (e.g. reservoir and hydraulic fracture characteristics, sorption features etc.) and operational constraint and the output is the gas production rate for all 169 clusters of hydraulic fractures.

Ten simulation runs cover different initial reservoir pressures, operational constraints, different isotherms and sorption features as well as different reservoir characteristics. In order to fully capture the shale gas production behavior as a function of time, three spatio-temporal databases for developing threes proxy models at cluster level with different time resolutions are developed (Daily for the first two months, monthly for the first 5 years and annually for 100 years).

Ten different shale regions with having unique sorption features such as Langmuir pressure, Langmuir volume, diffusion coefficient, sigma factor are defined in a way to include the equal number of clusters per region and shown in Figure 4-2.

In addition, corresponding methane isotherm for each region is illustrated in Figure 4-3.A complete list of inputs and corresponding ranges that are included in the data set is shown in Table 4-1.

Table 4-1.Main input data for Data-driven shale proxy models development

\begin{tabular}{|c|c|c|c|c|}
\hline $\begin{array}{c}\text { Matrix porosity } \\
{[0.054-0.135]}\end{array}$ & $\begin{array}{c}\text { Matrix permeability } \\
{[0.0001-} \\
0.00097(\mathrm{md})]\end{array}$ & $\begin{array}{c}\text { Natural fracture } \\
\text { porosity } \\
{[0.01-0.04]}\end{array}$ & $\begin{array}{c}\text { Natural fracture } \\
\text { permeability } \\
{[0.001-0.01(\mathrm{md})]}\end{array}$ & $\begin{array}{l}\text { Sigma factor } \\
{[0.005-0.6]}\end{array}$ \\
\hline $\begin{array}{c}\text { Hydraulic } \\
\text { fracture height } \\
{[100-125 \mathrm{ft}]}\end{array}$ & $\begin{array}{c}\text { Hydraulic fracture } \\
\text { length } \\
{[200-1100 \mathrm{ft}]}\end{array}$ & $\begin{array}{c}\text { Hydraulic fracture } \\
\text { conductivity } \\
{[0.5-5.4(\text { md-ft })]}\end{array}$ & $\begin{array}{c}\text { Rock Density } \\
{[100-180(1 \mathrm{~b} / \mathrm{ft} 3)]}\end{array}$ & $\begin{array}{l}\text { Net to Gross } \\
\text { ratio } \\
{[0.75-0.98]}\end{array}$ \\
\hline $\begin{array}{c}\text { Longmuir } \\
\text { volume } \\
{[40-85} \\
\text { (scf/ton)] }\end{array}$ & $\begin{array}{l}\text { Longmuir pressure } \\
\text { [600-870 psi] }\end{array}$ & $\begin{array}{c}\text { Diffusion coefficient } \\
{[0.5-2.8(\mathrm{ft} 2 / \text { day })]}\end{array}$ & $\begin{array}{l}\text { Sorption time } \\
{[1-250(\text { day })]}\end{array}$ & $\begin{array}{c}\text { Initial Reservoir } \\
\text { Pressure } \\
{[3000-4288(\mathrm{psi})]}\end{array}$ \\
\hline
\end{tabular}

In order to consider the possible changes in operational constraints and include them in the database, ten different bottom-hole pressure profile is designed with constant (200, 250, 300, 350 and 400 psi), increasing (from 150 to 550psi) and declining (from 2500 to 90 psi) trends. Figure 4-4 shows designed BHP profile changes for 100 years of production. 


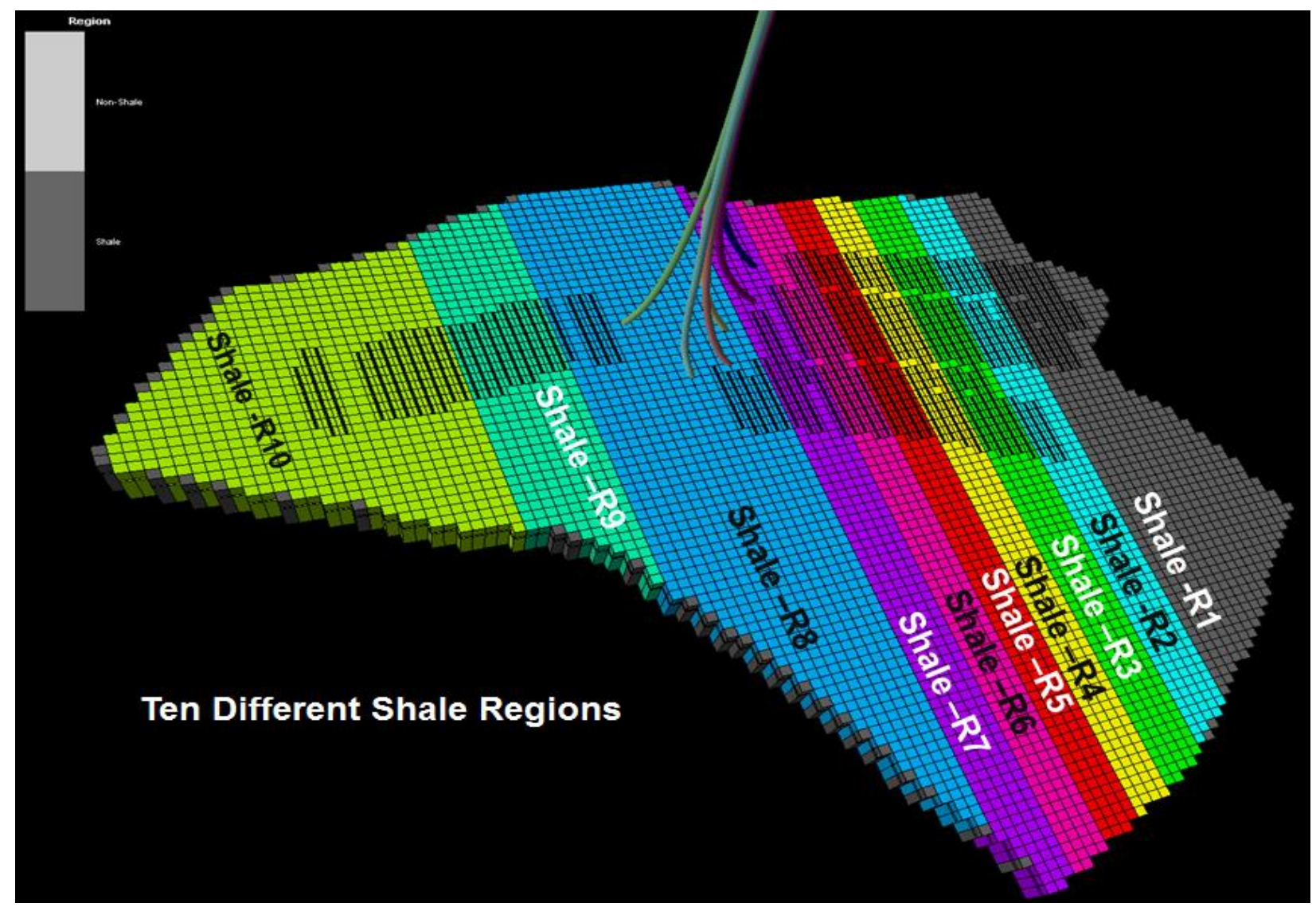

Figure 4-2. Ten different shale regions with unique sorption features

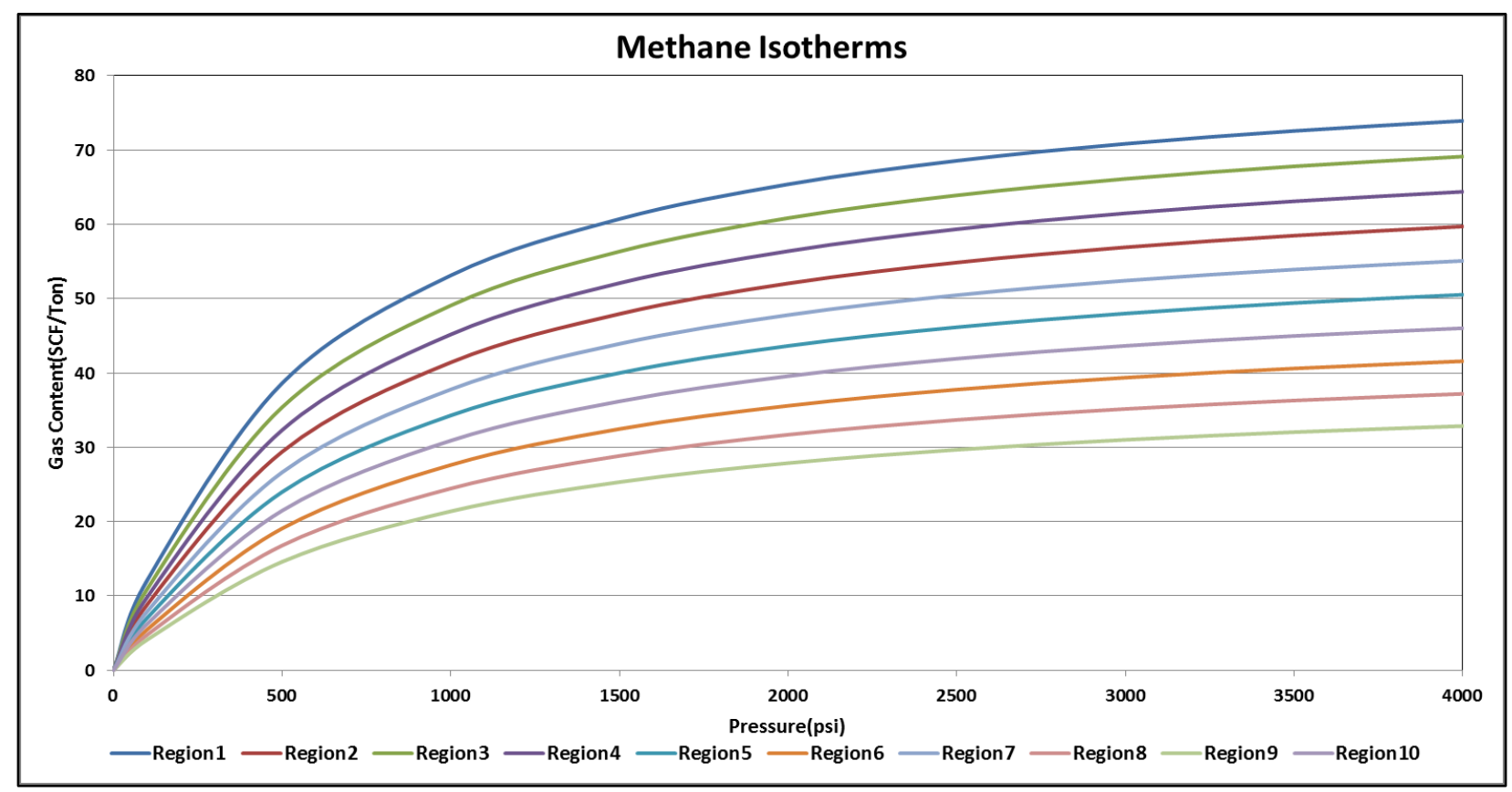

Figure 4-3. Ten different isotherms for ten different shale regions 


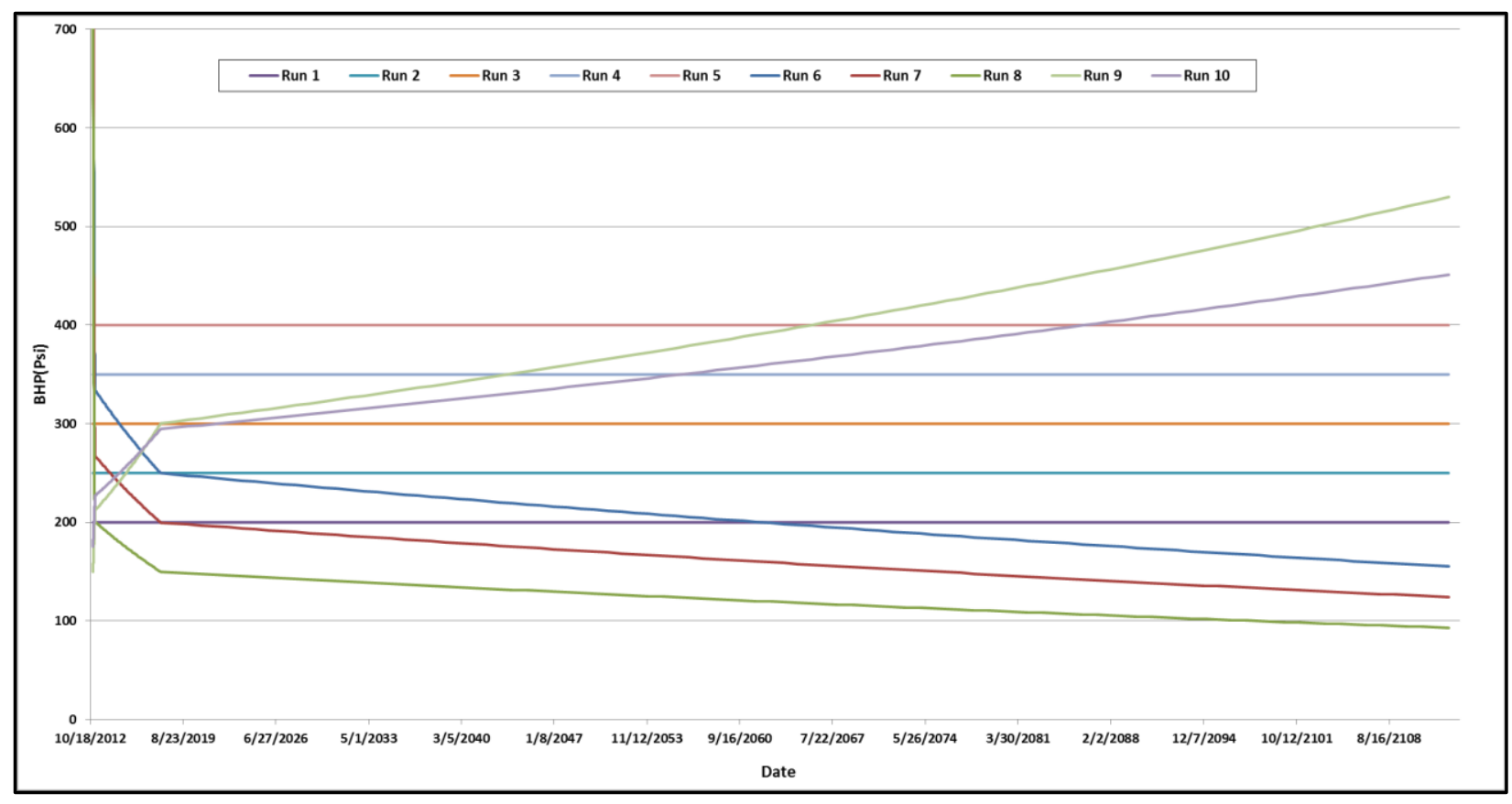

Figure 4-4. Different flowing bottomhole pressure profiles

It is important to realize that, since during the development of the Data-driven shale proxy model at the cluster level, the behavior of shale as a complex and non-linear system is taught to a computer using data, this information should cover the uncertainty domain and not to be the same (redundant). Therefore, in the case of dealing with homogenous reservoir, there is not much that can be taught to the neural network and more simulation runs are needed to get an acceptable result. On the other hand, even though having heterogeneous system, which is the case in this study, makes the process of building the Data-driven proxy model easier and requires less number of simulation runs, but it is impractical to include all grid block's properties in the spatio-temporal database.

In order to overcome this problem, a "Tiering system" is defined to take into account the impact of different grid blocks properties on each cluster production. Therefore, three different tiers are defined and a property for each tier is calculated by averaging the properties of all the grid blocks in the corresponding tier. Figure 4-5 (left) illustrates the Tiering systems and shows three types of tiers that are used in this study.

- Tier 1: Includes all the refined grid blocks for a target hydraulic fracture cluster. In other word, tier 1 covers the grid blocks that the hydraulic fracture cluster is extended horizontally and vertically and is equal to HF length and HF height. 
- Tier 2(- and +$)$ : Covers the rest of grid blocks that are extended to the north $(+)$ and south (-) of the tier 1, all the way toward the reservoir boundary or a hydraulic fracture from an offset lateral, both laterally and vertically.

- Tier 3(- and +$)$ : Covers the rest of grid blocks that are not covered by Tiers 1 and 2 .

Moreover, in order to teach the NNs, the interference effect between the clusters, they are divided into four classes based on their relative location respect to the other offset ones and is illustrated in Figure 4-5 (right).

- Type 1 Cluster: This type of cluster has only one neighboring cluster and therefore; it shares part of the drainage area.

- Type 2 Cluster: The second type of cluster has two neighboring clusters thus; the drainage area will be shared more than the type 1.

- Type 3 Cluster: Three neighboring clusters bound the third type thus; the drainage area will be shared more than type 1 and 2 .

- Type 4 Cluster: Four neighboring clusters bound the last type and drainage area will be shared more than the other 3 types.

Tier System Definition

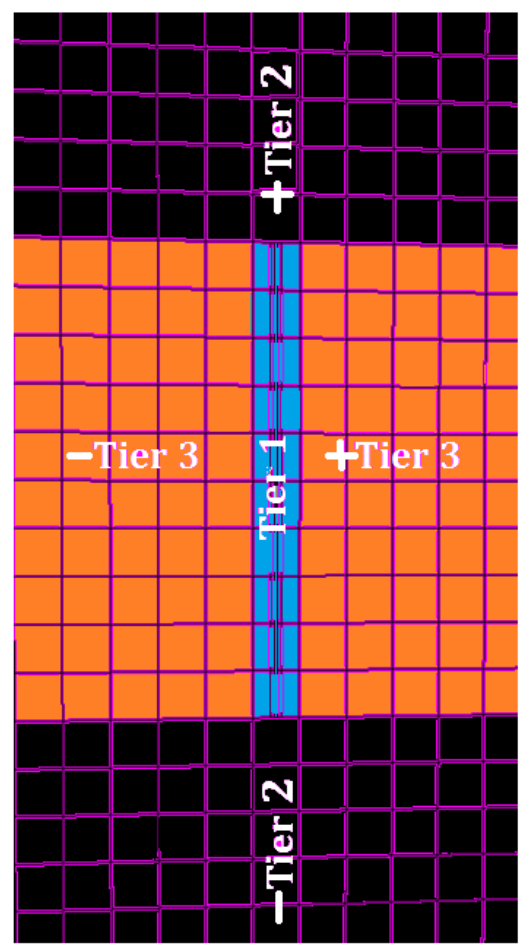

Cluster Type Definition

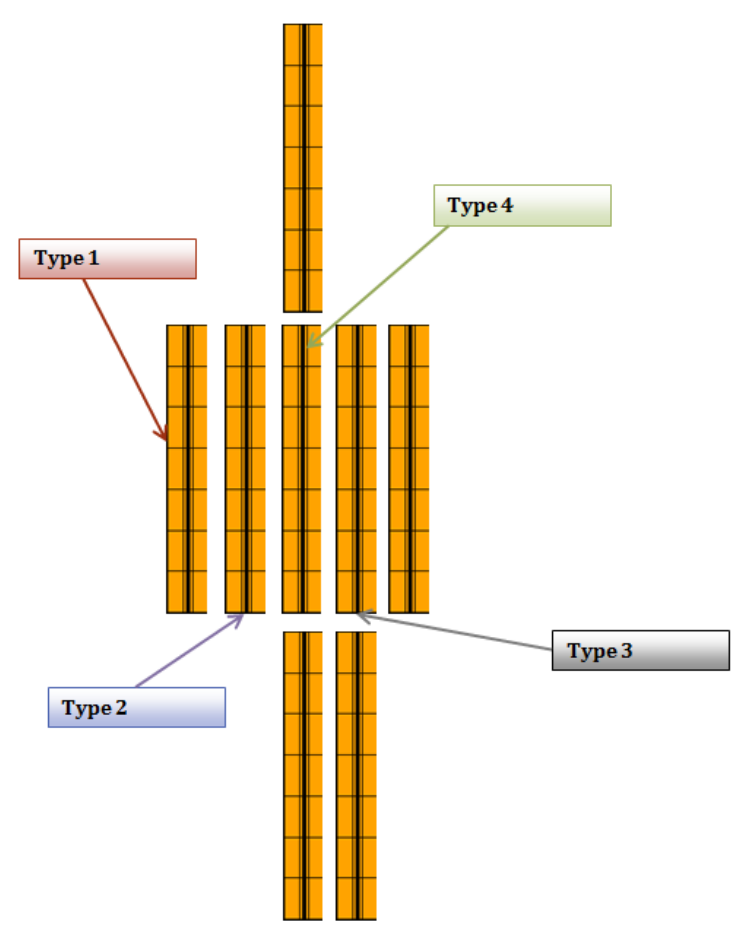

Figure 4-5.Tier system and cluster type definition (from left to right) 


\subsection{Data-Driven Proxy Model Development for Different Production Time Resolutions}

In this section, the results of three Data-driven shale proxy models at hydraulic fracture cluster level with different time resolutions are discussed. (Daily for the first two months, monthly for the first 5 years and annually for 100 years).

The developed Data-driven proxies are capable to generate the daily, monthly and annual gas production rate profile for each hydraulic fracture clusters as wells as corresponding laterals (Figure 4-6) (The summation of all the gas rates from all clusters generates a production profile for the corresponding horizontal lateral).

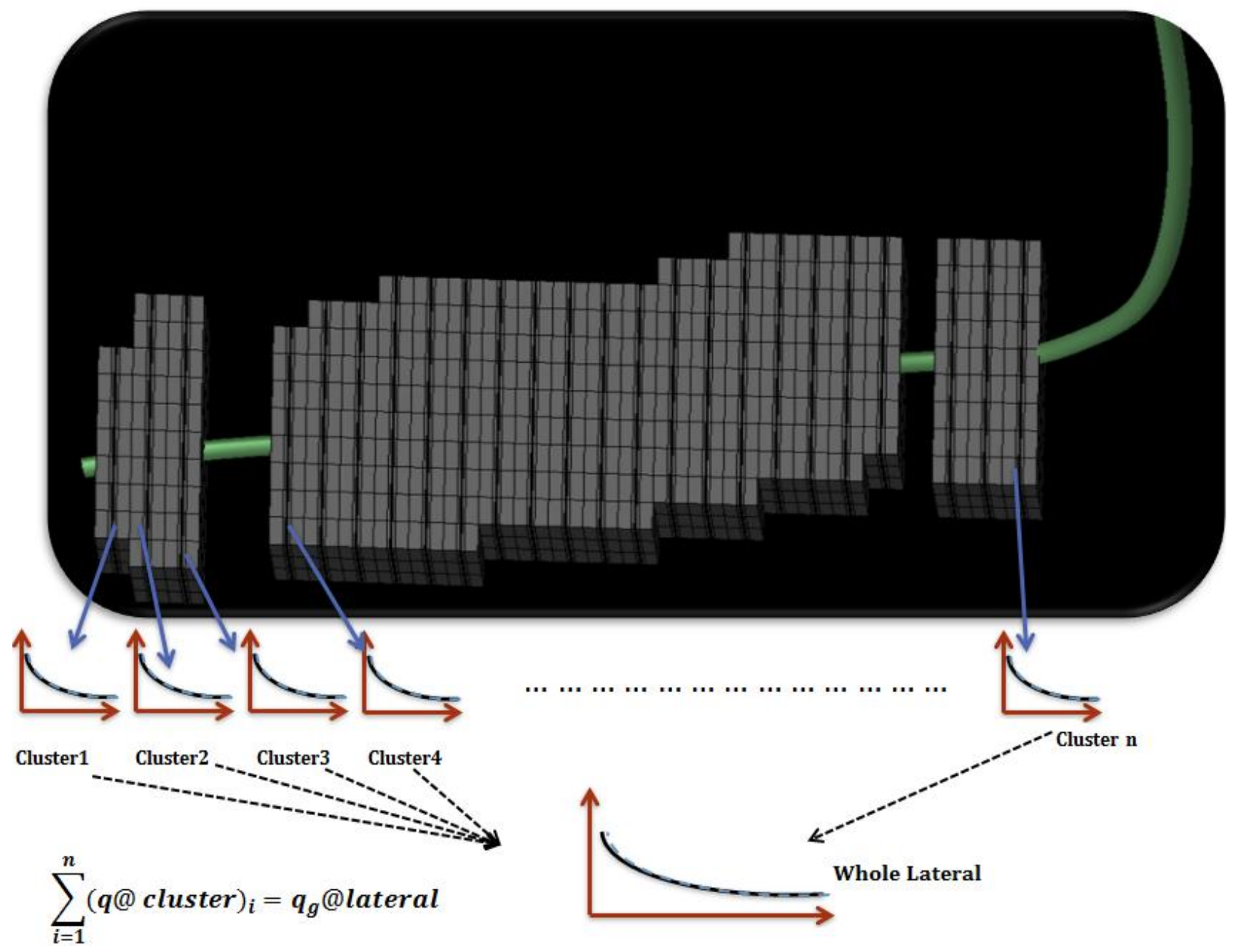

Figure 4-6. Illustration of generated production profile by Data-driven proxy model for each cluster and the corresponding lateral 


\subsubsection{Daily Production}

The first Data-driven shale proxy model is developed to re-generated daily gas production rate for the first two months of production that is already generated by Eclipse ${ }^{\mathrm{TM}}$ reservoir simulator, for each hydraulic fracture cluster. In order to do that, ten simulation runs are designed to generate 1690 unique (169 clusters* 10 runs) production profile with different reservoir characteristics, hydraulic fracture properties, sorption features (sorption time and Langmuir isotherms) and operational constraints.

The representative database with 98020 pairs of input-output is used to train a multilayer feed-forward back-propagation neural network, which is suitable for pattern recognitions especially in non-linear problems.

Key performance indicator (KPI) process is performed to rank the most influential input parameters on shale gas production. In this process, not only the impact of each input parameter but also influence of a combination of different inputs on monthly gas production can be identified.

A propriety algorithm (dubbed Fuzzy Pattern Recognition (Bezdeket al., 1992) is used to identify the influence of a combination of parameters. During Fuzzy Pattern Recognition (FPR), a curve is generated from the existing data. The slope of the curve determines the degree of influence of a parameter on the production indicator (the output - the target of the correlation). Therefore, the parameter with the largest slope is identified as the parameter with the highest influence on the output.

In order to simplify the analysis of the order of influence of parameters, the slopes are normalized giving the parameter with the highest slope the value of 100.Figure 4-7 shows the KPI analysis for daily database. According to this analysis, the hydraulic fracture conductivity and natural fracture permeability are among the most influential parameters for the first two months of production in the Marcellus shale.

Moreover, as it is expected, the sorption time and the Langmuir pressure and Langmuir volume which, controls the desorption, diffusion and adsorbed gas content of shale have the minimal impact on the first two months of production.

After completing the KPI analysis, the main part of the process of developing a proxy model can be started. Designed neural network for building daily Data-driven shale proxy model, has one hidden layers with 55 hidden neurons that are selected based on the number of data records available and the number of input parameters selected in each training process. 


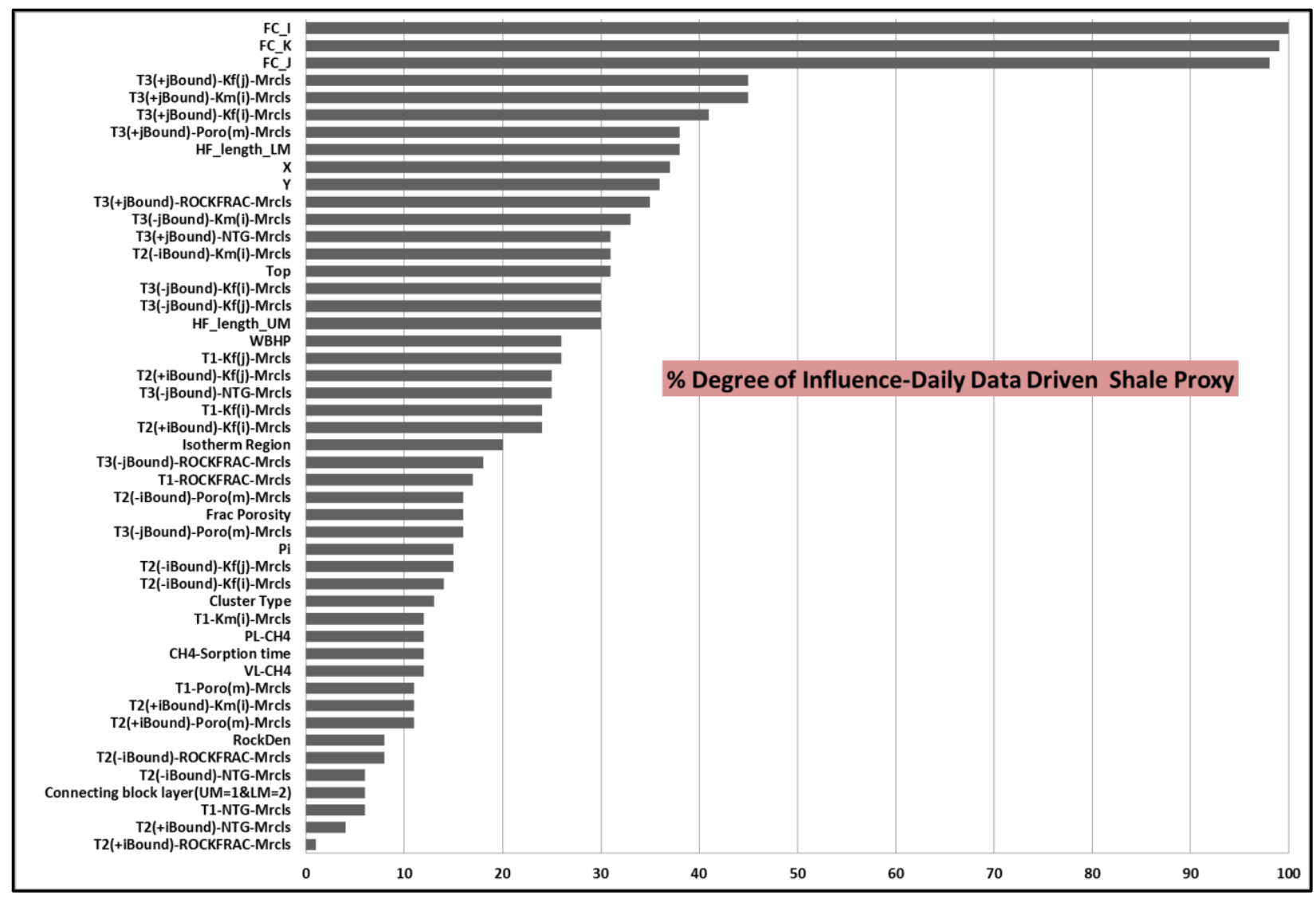

Figure 4-7. Key performance indicators based on the daily database for the first two months of production

In the training process, the data set is partitioned into three separate segments. This is done in order to make sure that the neural network will not be trapped in the memorization phase. The intelligent partitioning process allows the network to adapt to new data once it is being trained. The first segment, which includes the majority of the data, is used to train the model.

In order to prevent the memorizing and overtraining effect on the neural network training process, a second segment of the data is taken for calibration that is blind to the neural network and at each step of the training process, the network is tested for this set. If the updated network gives better predictions for the calibration set, it will replace the previous neural network; otherwise, the previous network is selected. Training will be continued once the error of predictions for both the calibration and training data set is satisfactory. This will be achieved only if the calibration and training partitions are showing similar statistical characteristics.

Verification partition is the third and last segment used for the process that is kept out of training and calibration process and is used only to test the precision of the neural networks. Once a network is trained 
and calibrated, then the final model is applied to the verification set. If the results are satisfactory then the neural network is accepted as part of the entire prediction system.

It should be noted that using all the data for training-calibration and verification process might not always lead to a successful trained NN. In other word, the process can be started with maximum number of inputs and based on the modeler's reservoir engineering judgments and understanding of fluid flow in shale and also the training results, this number can be reduced to come up with the optimum number of inputs to achieve the best training-calibration and verification results.

Table 4-2 summarizes the list of optimum inputs (35 inputs) that are used for development of daily basis shale proxy model.

Table 4-2. List of optimum number of input for developing Data-driven proxy model (Daily basis)

\begin{tabular}{|c|c|c|}
\hline \multicolumn{3}{|c|}{ Daily NN- Input Parameters } \\
\hline \multirow{24}{*}{ Static Inputs } & \multirow{14}{*}{$\begin{array}{c}\text { Cluster/Reservoir } \\
\text { Characteristics }\end{array}$} & Location $(X, Y)$ \\
\hline & & Top \\
\hline & & Cluster Type \\
\hline & & T1-Km(I)-Marcellus \\
\hline & & T2(-iBound)-Km(I)-Marcellus \\
\hline & & $\mathrm{T} 2(+\mathrm{iBound})-\mathrm{Km}(\mathrm{I})$-Marcellus \\
\hline & & T3(-jBound)-Km(I)-Marcellus \\
\hline & & T3(-jBound)-Km(I)-Marcellus \\
\hline & & T1-Porosity-Marcellus \\
\hline & & T2(-iBound)-Porosity-Marcellus \\
\hline & & T2(+iBound)-Porosity-Marcellus \\
\hline & & T3(-jBound)-Porosity-Marcellus \\
\hline & & T3(-jBound)-Porosity-Marcellus \\
\hline & & Initial Reservoir Pressure \\
\hline & \multirow{4}{*}{ HF Properties } & Fracture Conductivity in $\mathrm{I}, \mathrm{J}, \mathrm{K}$ \\
\hline & & \# LGR blocks_UM \\
\hline & & \# LGR blocks_UM \\
\hline & & Connection Block Layer (UM=1, LM=2) \\
\hline & \multirow{6}{*}{$\begin{array}{c}\text { Natural Fracture } \\
\text { Properties }\end{array}$} & T1-Kf(I,k)-Marcellus \\
\hline & & T2(-iBound)-Kf(I,k)-Marcellus \\
\hline & & T2(+iBound)-Kf(I,k)-Marcellus \\
\hline & & T3(-jBound)-Kf(I,k)-Marcellus \\
\hline & & T3(-jBound)-Kf(I,k)-Marcellus \\
\hline & & Porosity \\
\hline \multirow{2}{*}{ Dynamic Input } & \multicolumn{2}{|r|}{ Time } \\
\hline & \multicolumn{2}{|c|}{ Well Bottomhole Pressure } \\
\hline \multicolumn{3}{|c|}{35 Inputs } \\
\hline
\end{tabular}



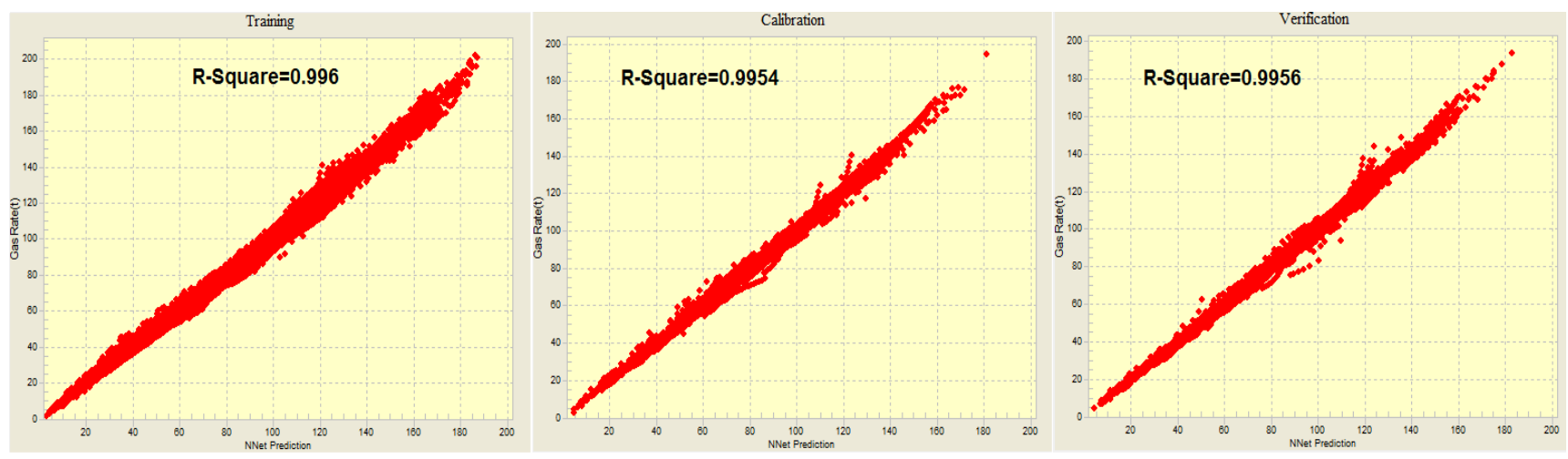

Figure 4-8. NN training, calibration and verification results (From left to right relatively)- Daily proxy model

Data are partitioned with a $80 \%$ training fraction, $20 \%$ for calibration and verification ( $10 \%$ for each). The cross plots for predicted and simulated values of daily gas flow rate (Mscf/day) for training, calibration and verification steps (from left to right) are shown in Figure 4-8. In these plots, $x$-axis corresponds to the neural network predicted gas rate and the y-axis shows the simulated gas rate by reservoir simulator (Eclipse). The result with an $\mathrm{R}^{2}$ of more than 0.99 in all steps shows the successful development of daily basis Data-driven shale proxy model.

Figure 4-9 and Figure 4-10 show some examples of the comparison of reservoir simulation output for daily gas production rate (Mscf/day) with the predicted one by Data-driven proxy model for some of the clusters as well as some of the laterals. The rest of the results are shown in Appendix A. It should be noted that, Data-driven proxy model, AI-based proxy model and Cluster based surrogate reservoir model (CSRM) are used in this dissertation interchangeably.

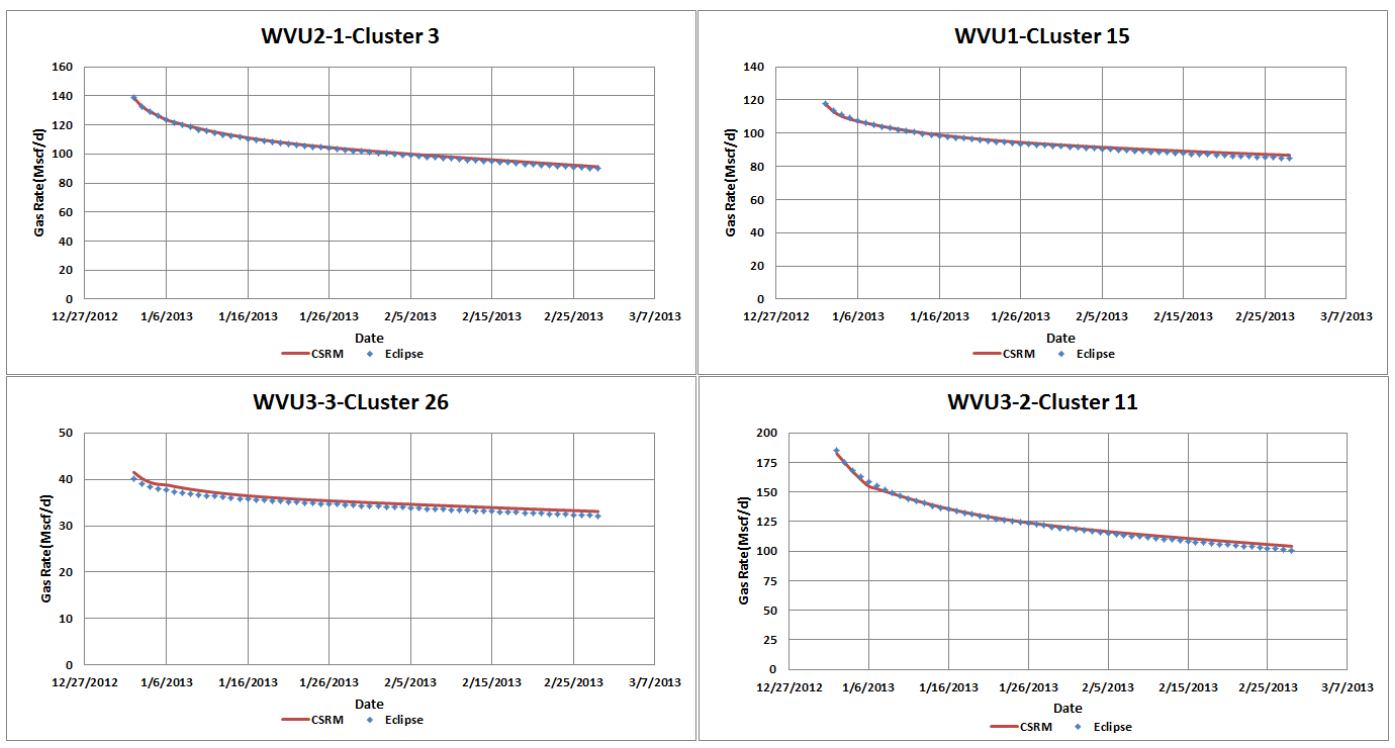

Figure 4-9. Comparison of gas production profile from simulator and Data-driven proxy model for some of the clusters 


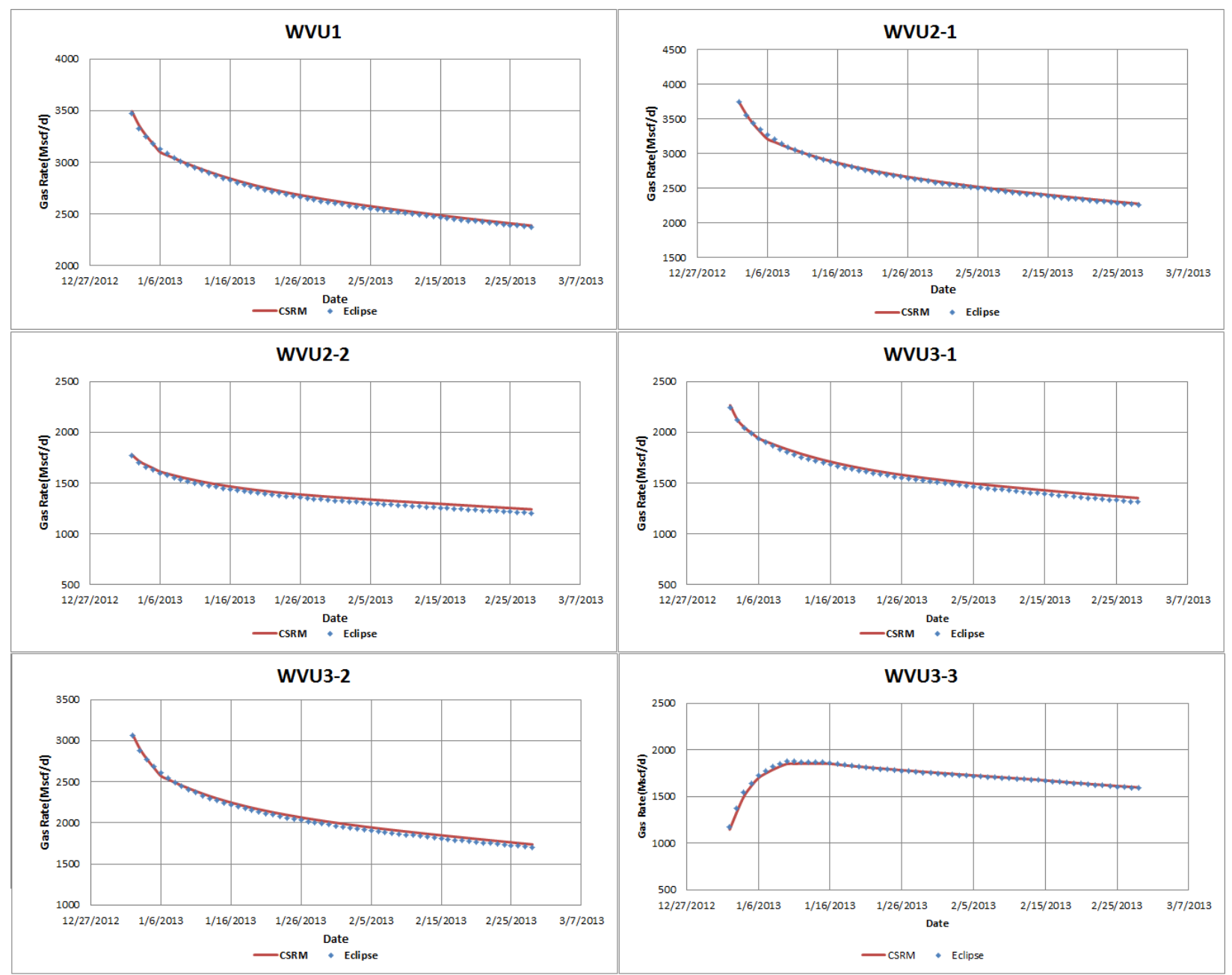

Figure 4-10. Comparison of gas production profile from simulator and Data-driven proxy model for some of the laterals

In all the plots blue dots represent the daily gas rate generated by Eclipse and the solid red line is the result of Data-driven shale proxy model. The results are self-descriptive enough to show the capability of Data-driven proxy model in predicting the daily gas production profile for each hydraulic fracture cluster as well as lateral for the first two months of production. Moreover, the predicted production rate at cluster level can be accounted as synthetic PLT log at each time step.

\subsubsection{Monthly Production}

Second Data-driven proxy model is developed to re-generate the monthly gas rate production (Mscf $/ \mathrm{m})$ for the first five years of production. The same procedure for building the first proxy (daily basis) is followed here too. In order to identify the impact of each parameter on monthly gas production (for the first five years) KPI analysis is performed. Figure 4-11 shows the KPI analysis for monthly database. It is interesting that time is the most influential parameter on five years of production. 


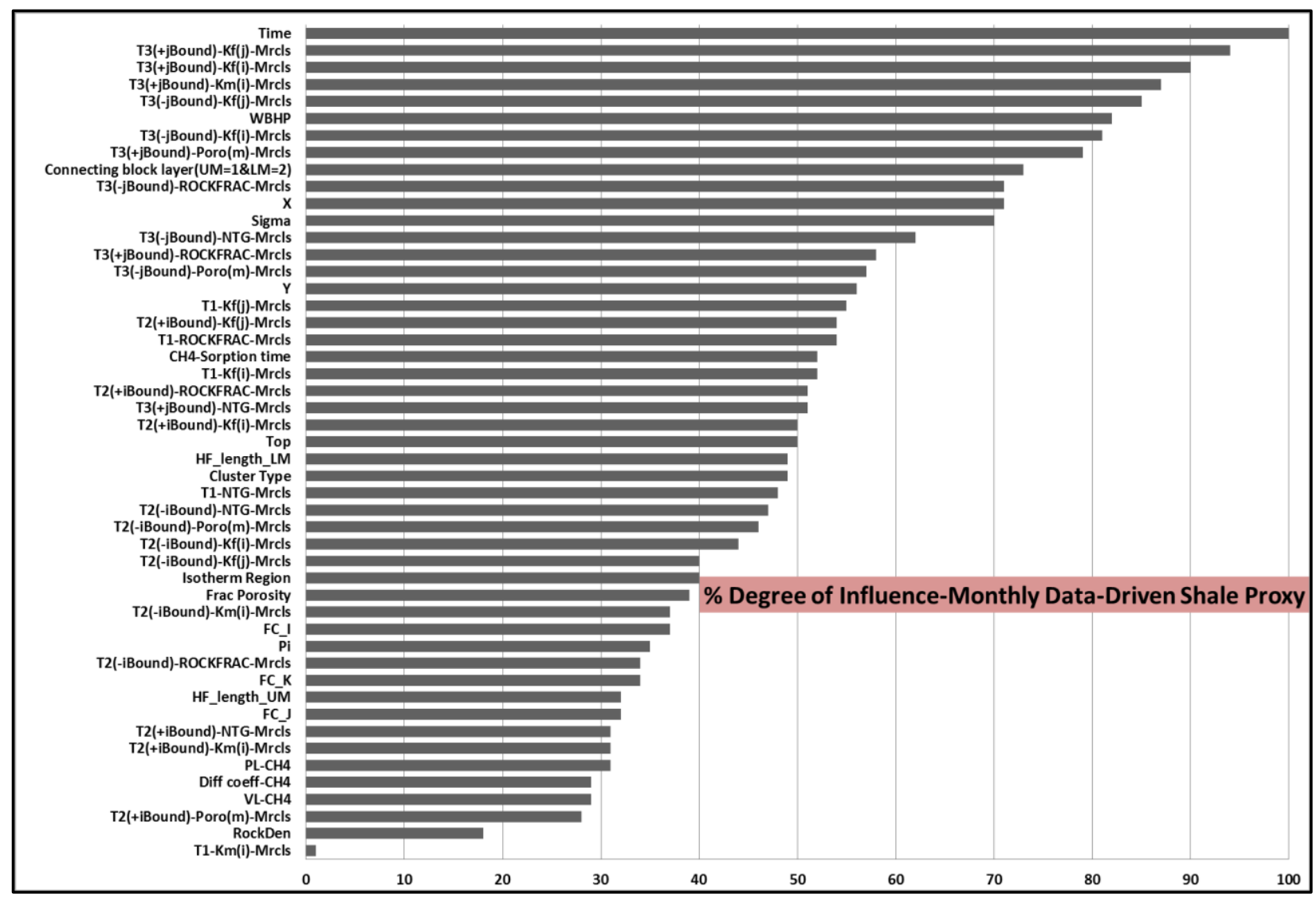

Figure 4-11. Key performance indicators based on the monthly database for the first five years of production

The other parameters such as natural fracture permeability, flowing bottomhole pressure as well as porosity are among the highest ranked parameters. Another interesting point by looking at KPI behavior are high ranking of hydraulic fracture cluster location, which accounts for the interference between the clusters and also high impact of sigma factor that control matrix to fracture flow. Alternatively, very low ranking of Langmuir volume and Langmuir pressure as well as diffusion coefficient and sorption time confirm the insignificant impact of sorption features on production performance for the first five years of production.

Table 4-3 summarizes the list of optimum inputs (49 inputs) that are used for development of monthly basis shale proxy model.

The representative database with 101,400 pairs of input-output is used to train a multilayer feed-forward back-propagation neural network. Before starting the training process, the data is partitioned with $80 \%$ training, and $20 \%$ for calibration and verification. The neural network has one hidden layers with 55 hidden neurons. 
Table 4-3. List of optimum number of input for developing Data-driven proxy model (Monthly basis)

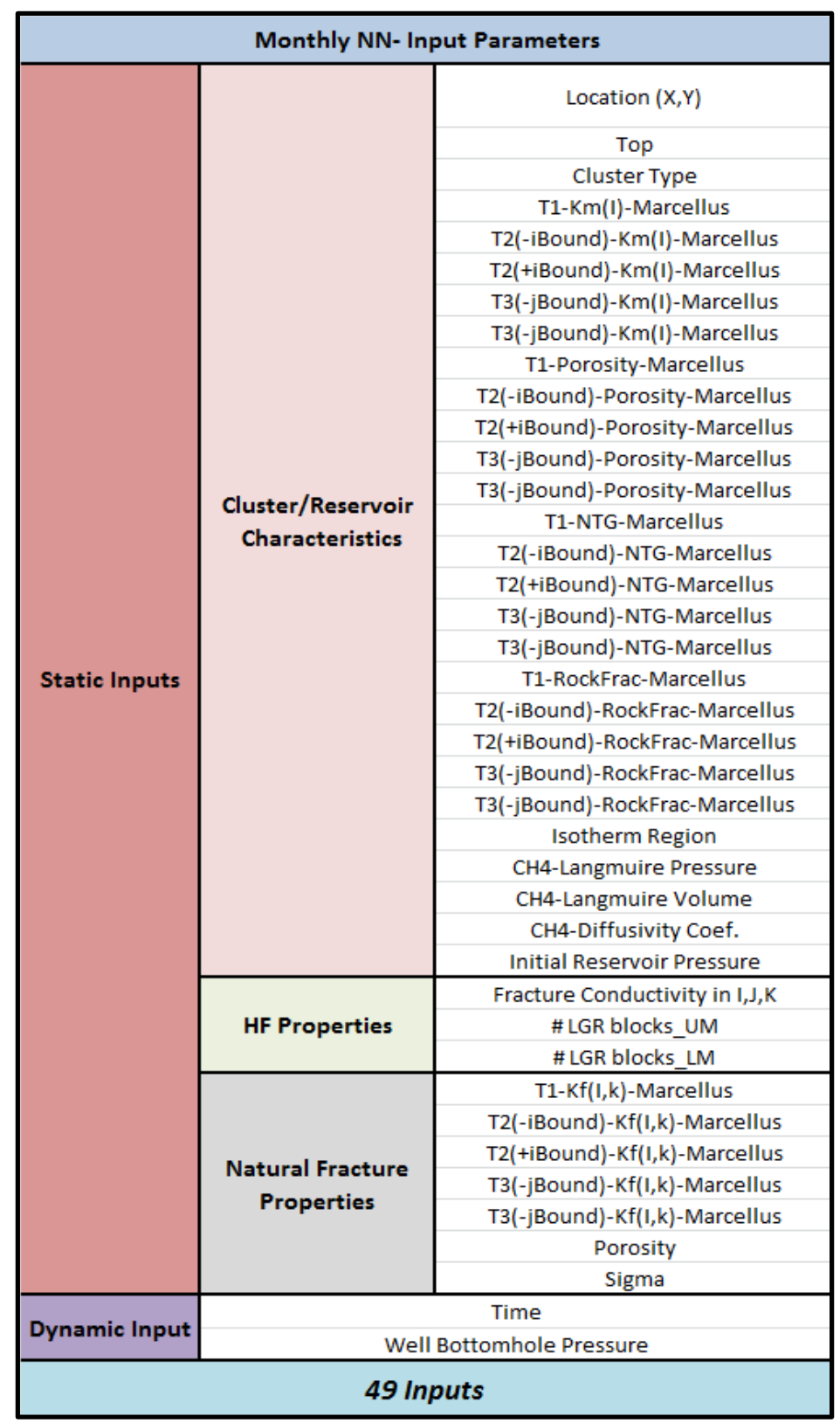

The cross plots for predicted and simulated values of monthly gas flow rate (Mscf/month) for training, calibration and verification steps (from left to right) are shown in Figure 4-12. In these plots, x-axis corresponds to the neural network predicted gas rate and the y-axis shows the simulated gas rate by Eclipse.

In all the steps (training, calibration and verifications), $\mathrm{R}^{2}$ of more than 0.99 shows the successful development of monthly basis Data-driven shale proxy model. Figure 4-13 and Figure 4-14 show some 

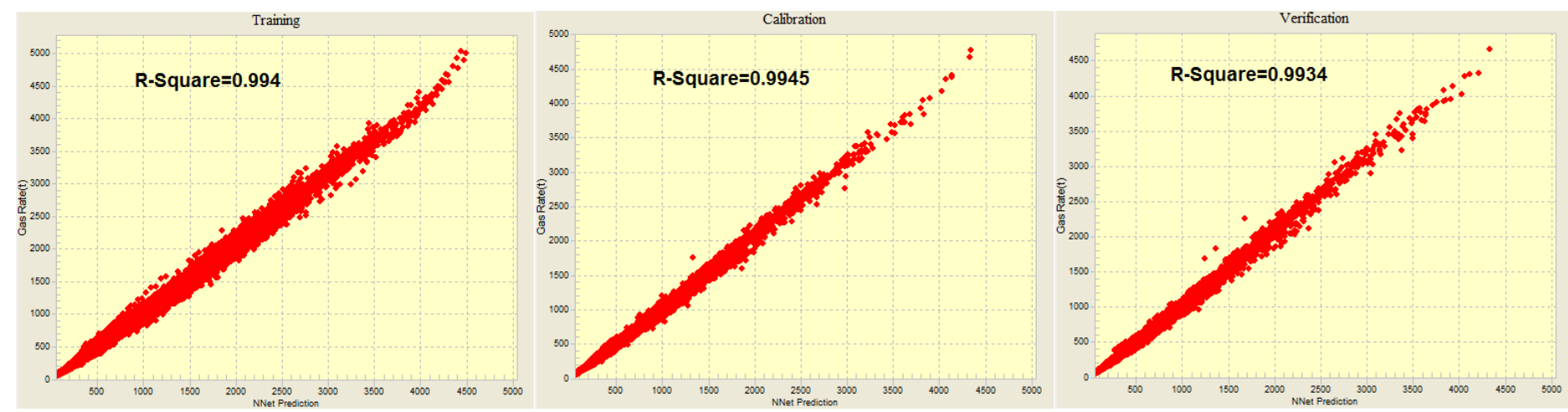

Figure 4-12.NN training, calibration and verification results (From left to right relatively)-Monthly proxy model

examples of the comparison of reservoir simulation output for monthly gas production rate with the predicted one by Data-driven proxy model for some of the clusters as well as some of the laterals. The rest of the results are shown in Appendix A-2.

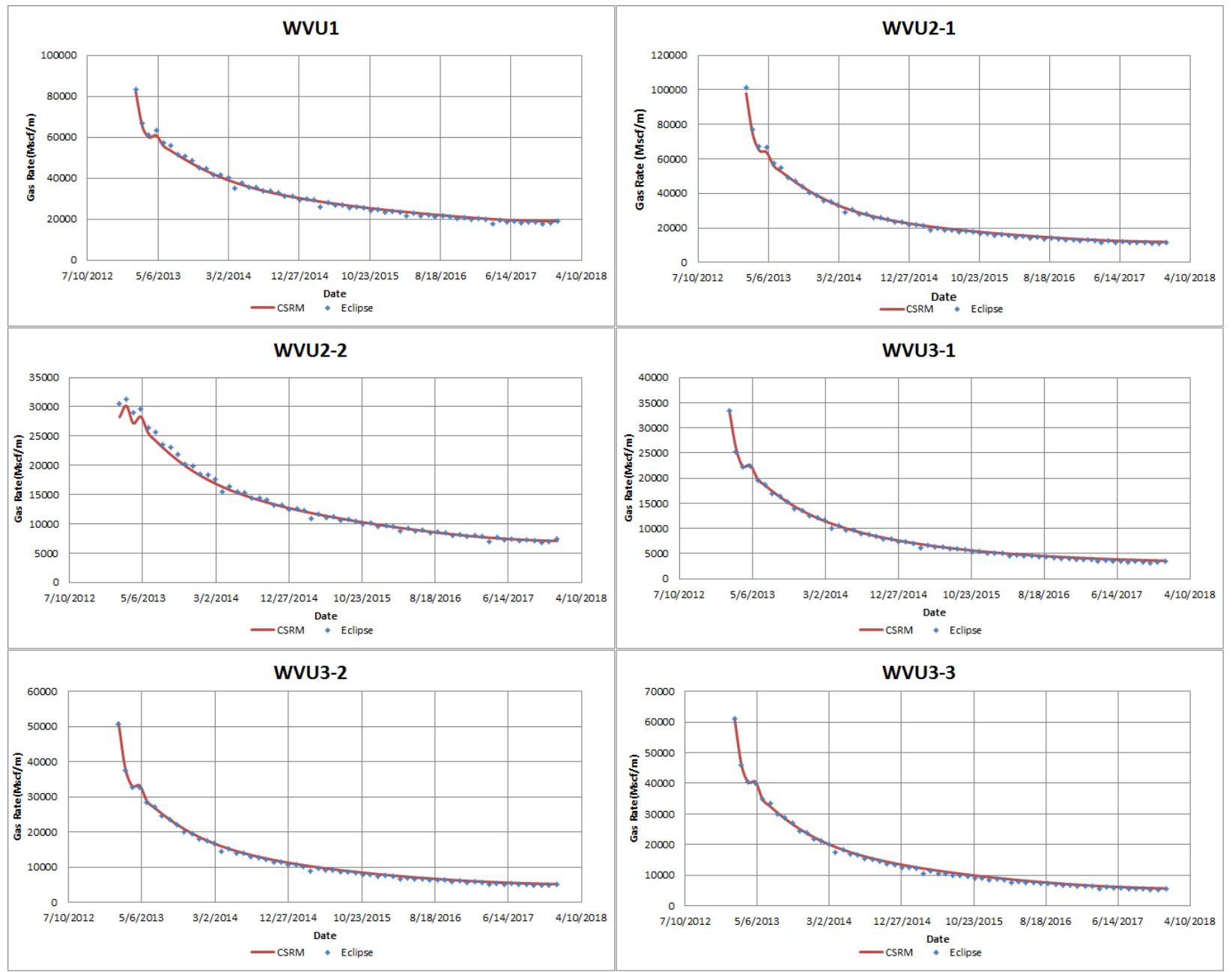

Figure 4-13. Comparison of gas production profile from simulator and Data-driven proxy model for some of the laterals 


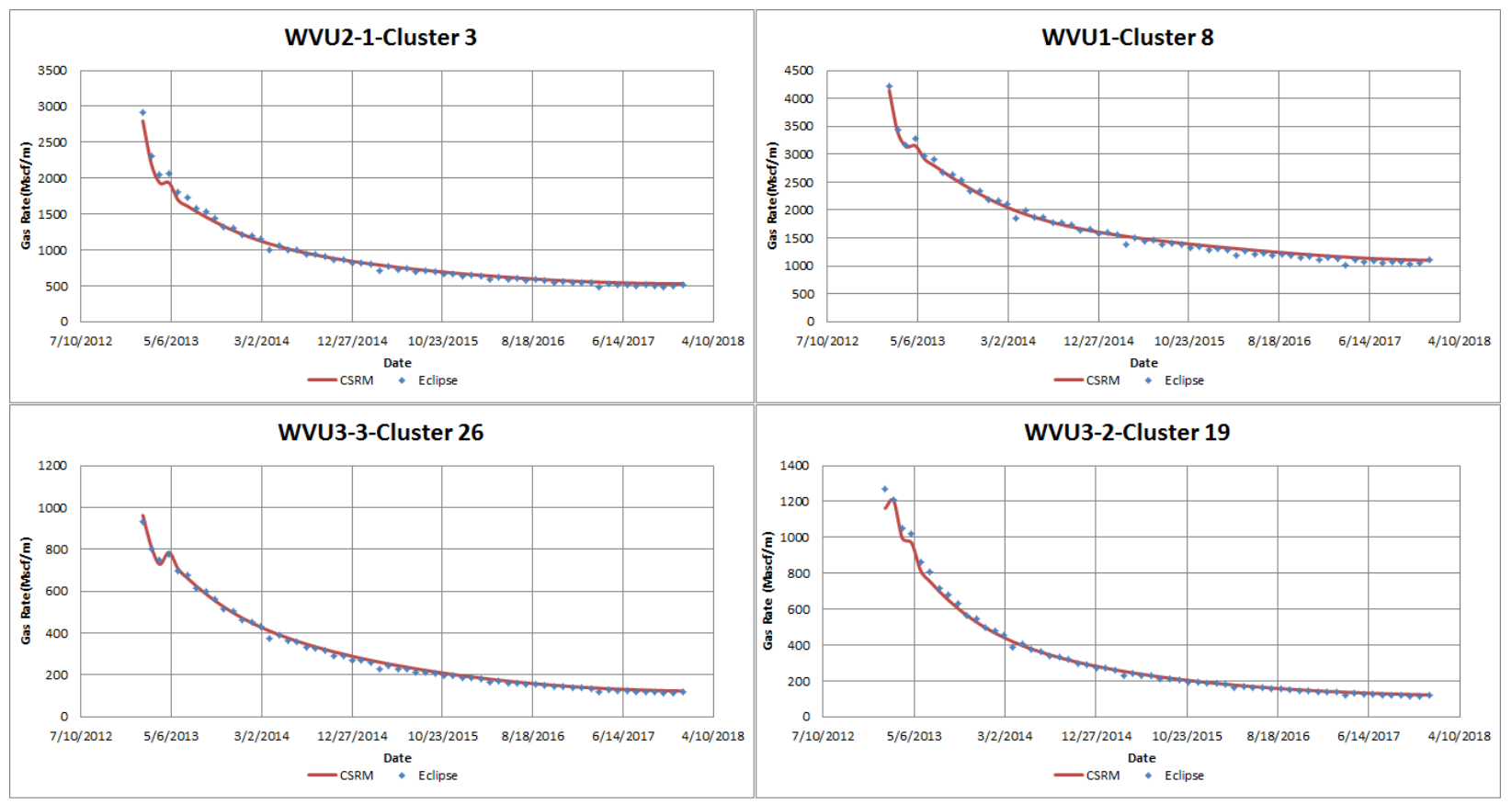

Figure 4-14. Comparison of gas production profile from simulator and Data-driven proxy model for some of the clusters

As can be seen from Figure 4-13 and Figure 4-14, Data-driven proxy model is re-produced the monthly gas rate production profile, which is generated by Eclipse for the first five years of production at cluster and the lateral level with high accuracy. In all the plots, blue dots represent the monthly gas rate generated by Eclipse and the solid red line demonstrates the result of the shale proxy model.

\subsubsection{Annul Production}

The last Data-driven shale proxy model is developed to re-generate the annual gas rate production (Mscf/year) for 100 years of production. The same procedure for the development of previous proxies is followed here as well. In order to see the impact of each parameter on annual gas production (for 100 years) KPI analysis is performed. Figure 4-15shows the KPI analysis for annual database. Production time, sorption time and natural fracture and matrix permeability are the most influential parameters on 100 years production performance of shale gas well.

Table 4-4summarizes the list of optimum inputs (44 inputs) that are used for development of annual basis shale proxy model.

The descriptive spatio-temporal database with 169,000 pairs of input-output is used to train a multilayer feed-forward back-propagation neural network. The data set is partitioned with $80 \%$ training, and $20 \%$ for calibration and verification. The neural network has one hidden layers with 55 hidden neurons. 


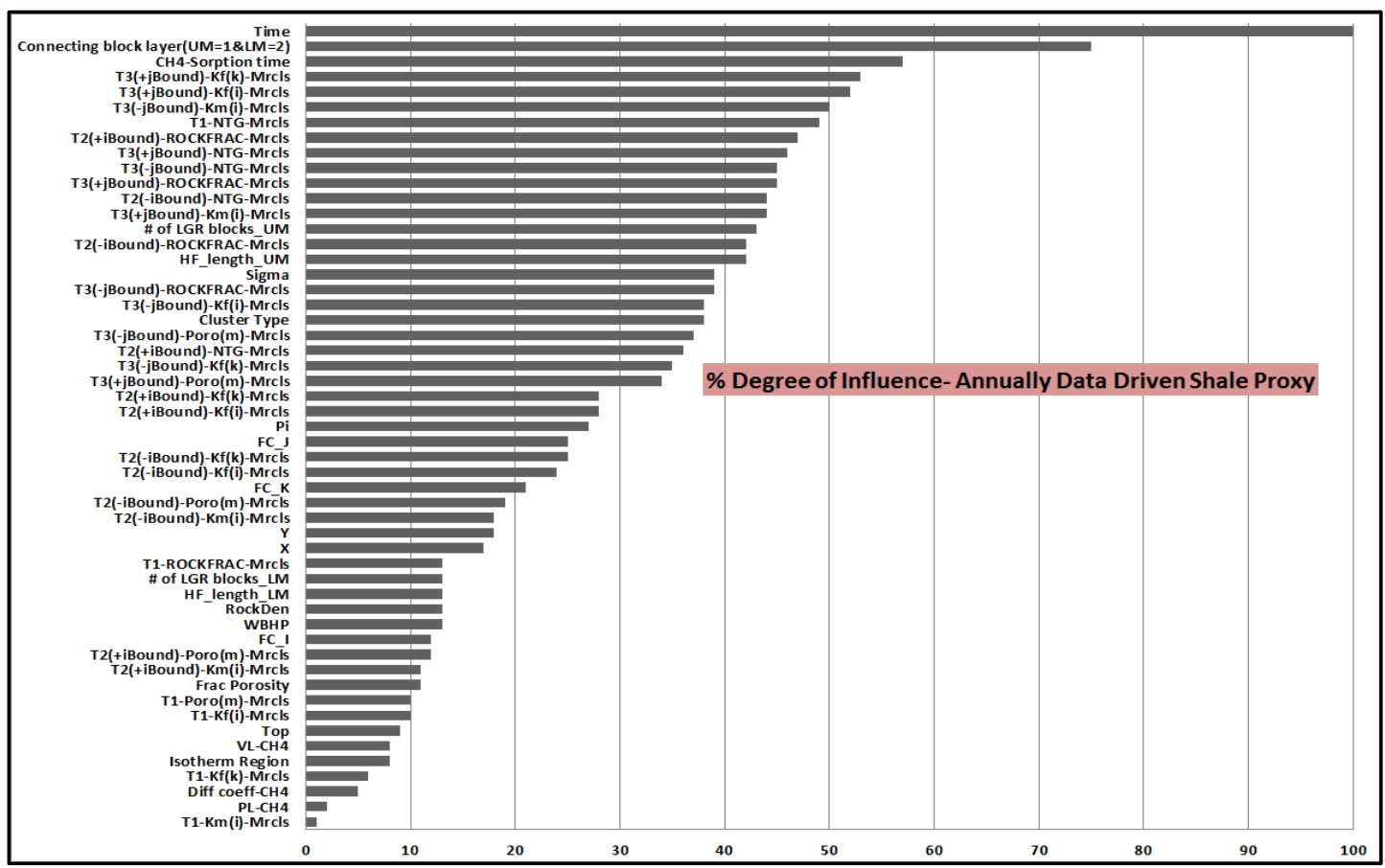

Figure 4-15. Key performance indicators based on the monthly database for 100 years of production

Table 4-4. List of optimum number of input for developing Data-driven proxy model (Annual basis)

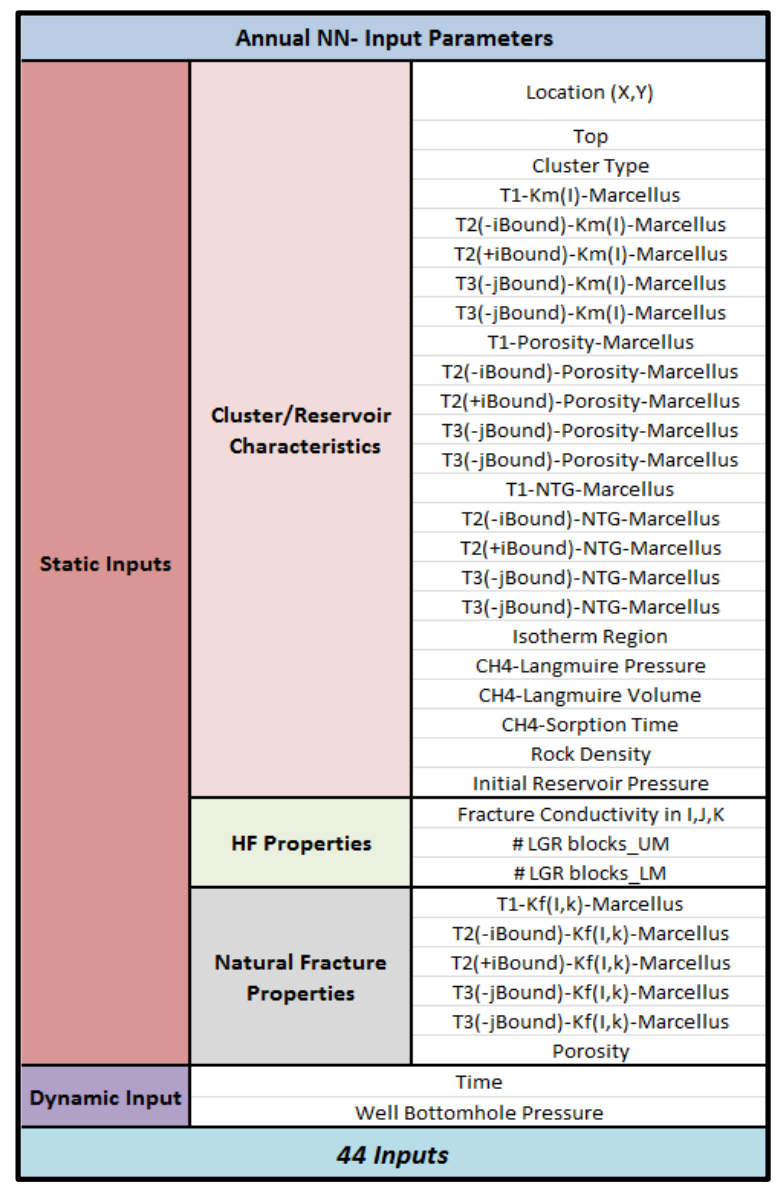



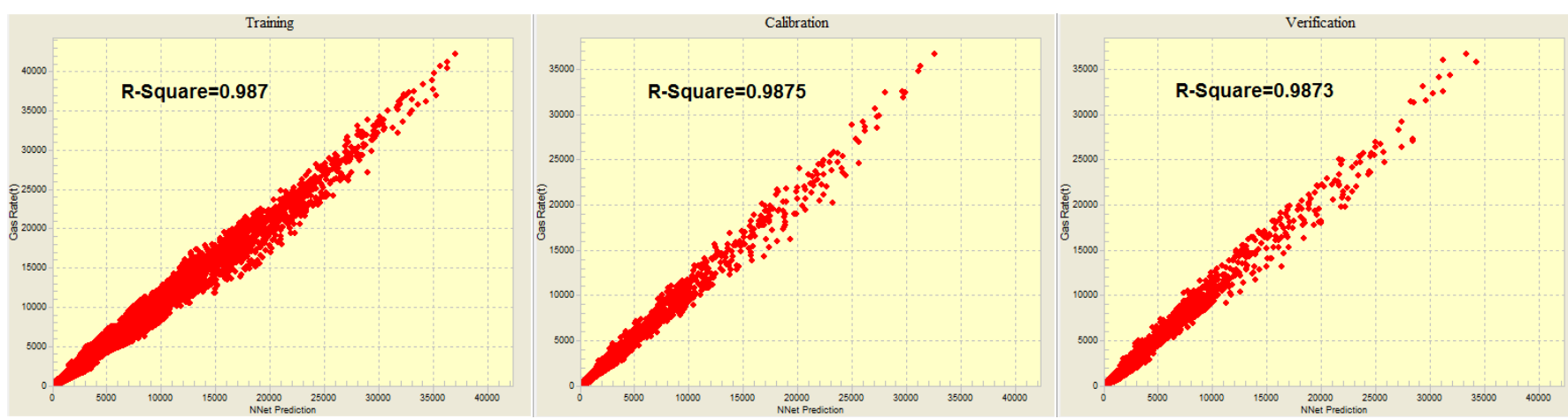

Figure 4-16. NN training, calibration and verification results (From left to right relatively)-Annual proxy model

Figure 4-16 illustrates the cross plots for predicted and simulated values of annual gas flow rate (Mscf/year) for training, calibration and verification steps (from left to right).In these plots, $\mathrm{x}$-axis corresponds to the neural network predicted gas rate and the y-axis shows the simulated gas rate by Eclipse. The calculated $\mathrm{R}^{2}$ for training, calibration and verification results are around 0.99 .

Several examples of the comparison of reservoir simulation output for annual gas production rate with the predicted one by Data-driven proxy model for some of the clusters and the laterals are shown in Figure 4-17 and Figure 4-18. The rest of the results are shown in Appendix A-3.In all the plots, blue dots represent the annual gas rate generated by Eclipse while the solid red line shows the proxy model result.

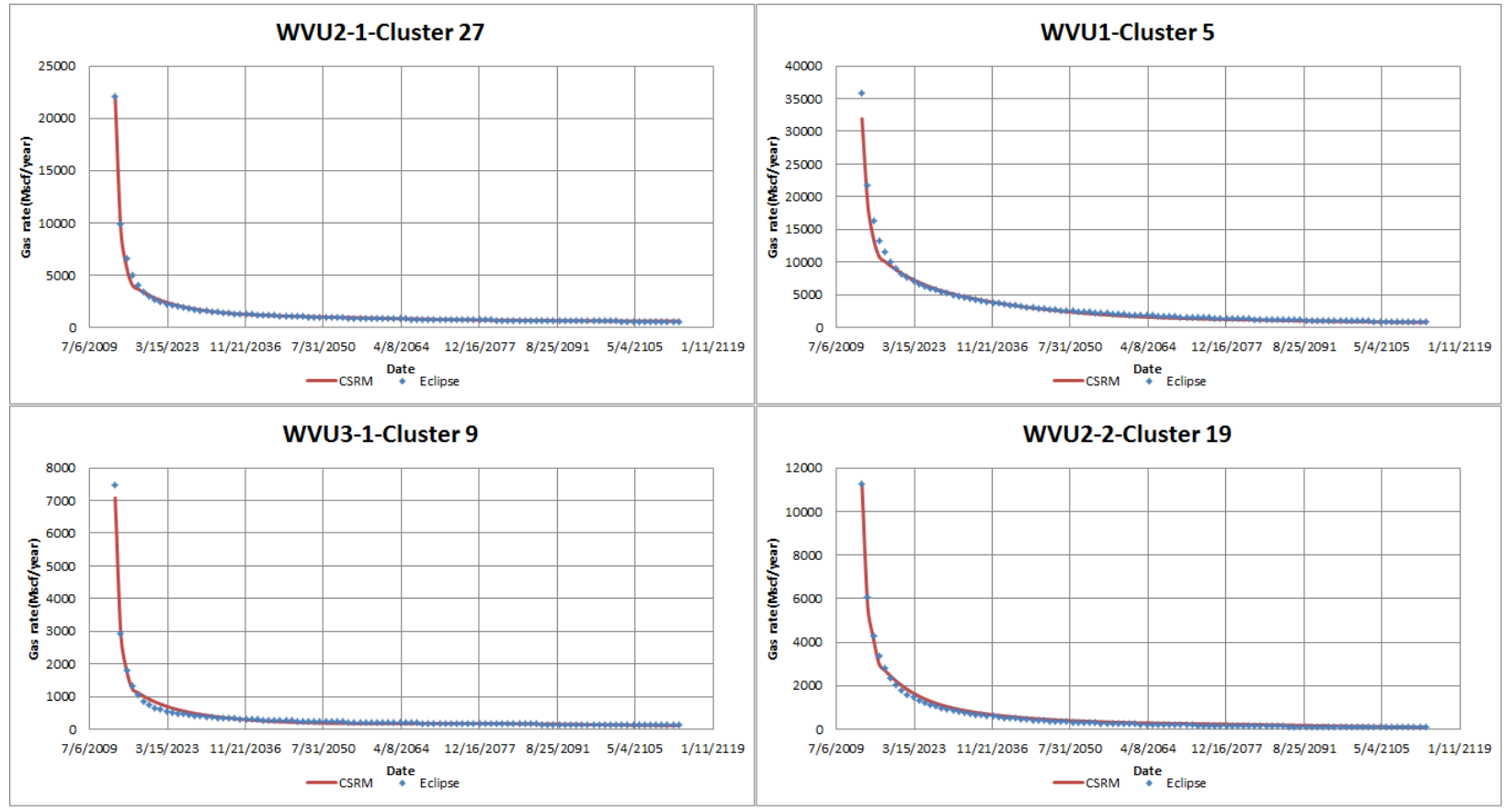

Figure 4-17. Comparison of gas production profile from simulator and Data-driven proxy model for some of the clusters 


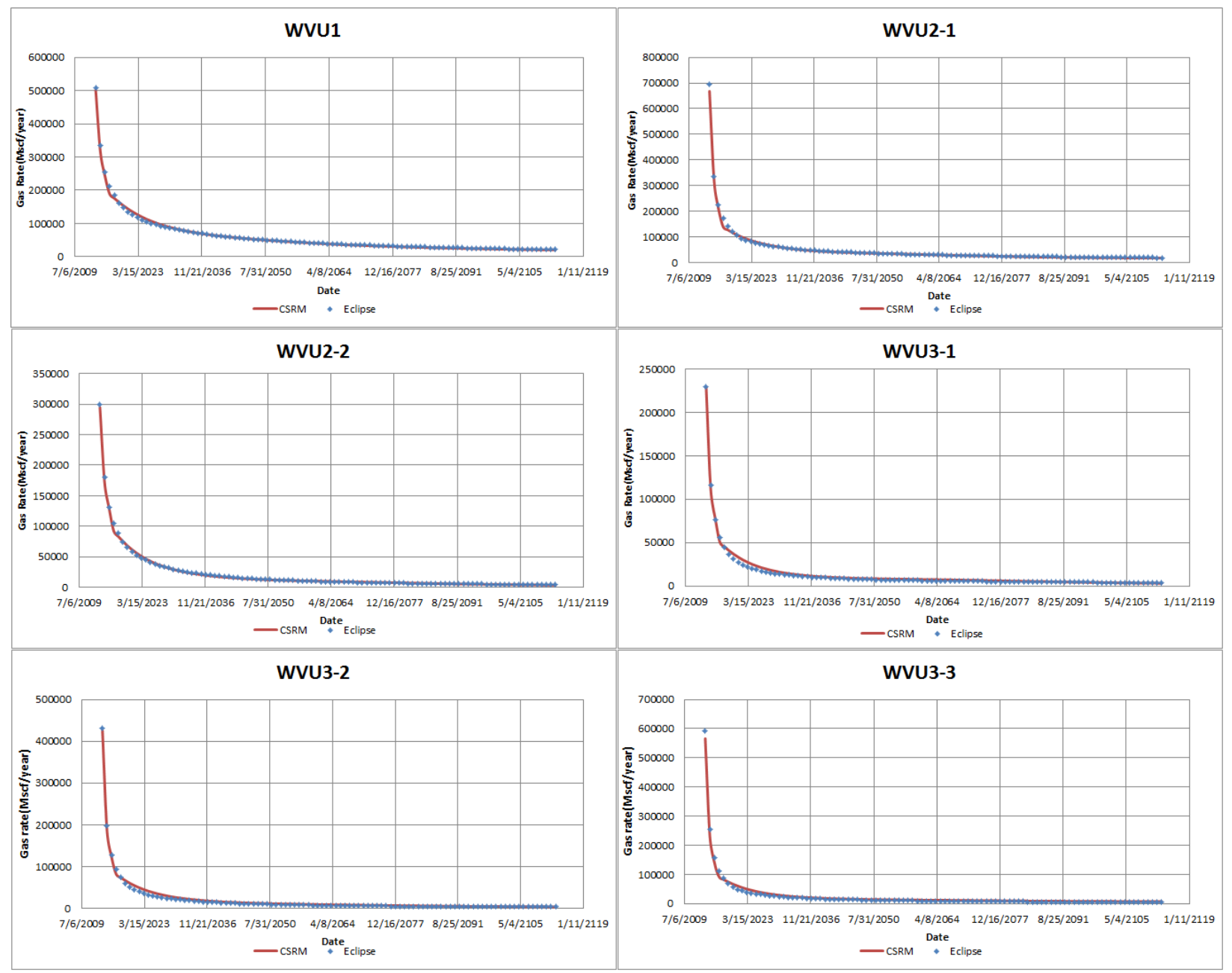

Figure 4-18. Comparison of gas production profile from simulator and Data-driven proxy model for some of the laterals

As illustrated in Figure 4-17 and Figure 4-18, Data-driven proxy model is successfully re-generated annual gas rate production profile (Red solid line) that generated by Eclipse ( Blue dots) for the 100 years of production at cluster and lateral level.

The only problem that can be observed in almost all the cases is that the annual based proxy model could not capture the transient behavior that is happening during the first five years of production. In order to address this problem, monthly and annual based shale proxies are combined. In other word, the first five years of production in annual proxy is replaced by the corresponding values that already generated by monthly proxy model. In this case, the results are improved significantly.

Figure 4-19 and Figure 4-20 show this improvement for four clusters as an example (Figure 4-19) and the whole WVU2-2 lateral as well (Figure 4-20). 

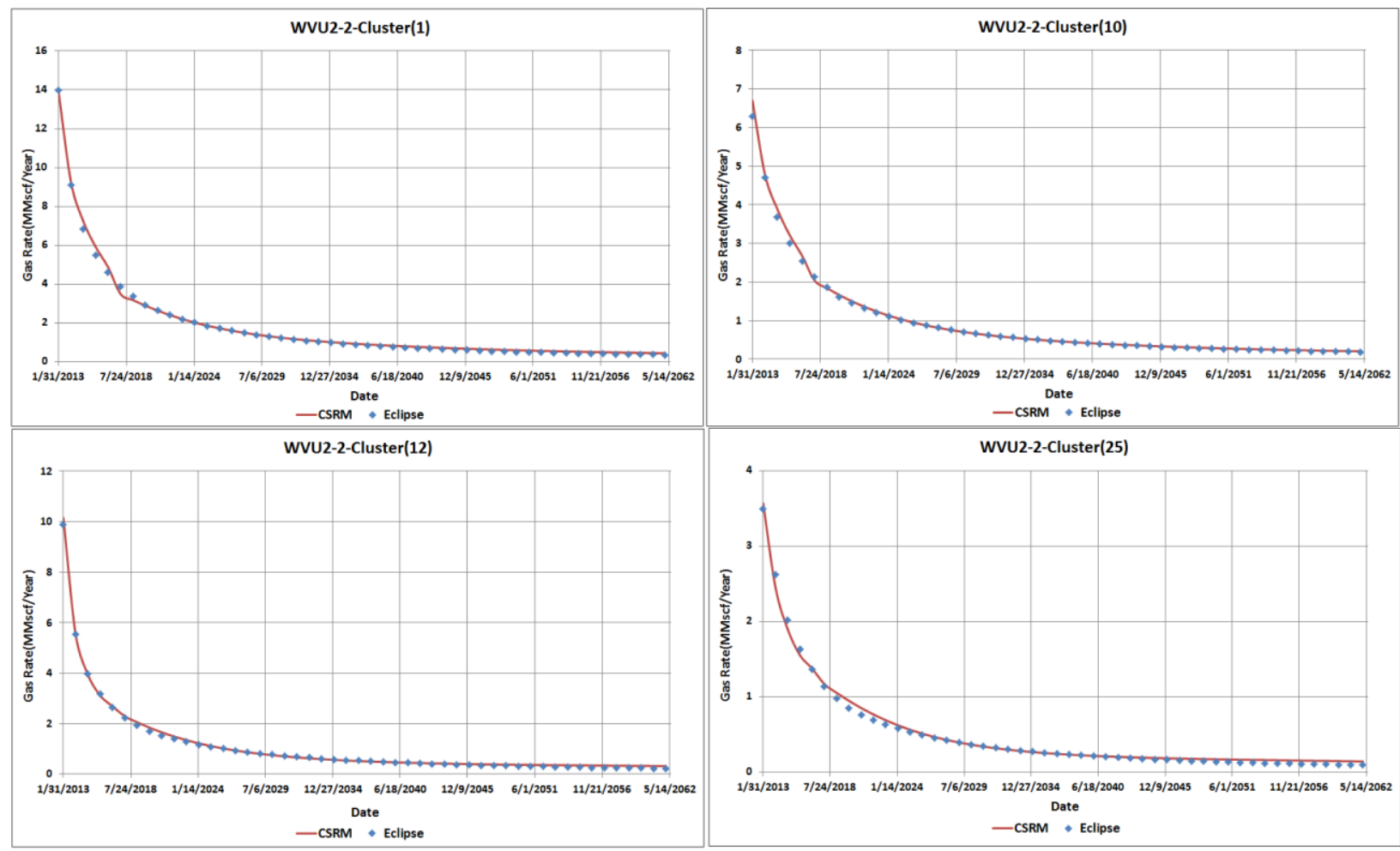

Figure 4-19. Combined monthly and annual proxies- Annual gas rate production for four clusters located in WVU-2-2 lateral

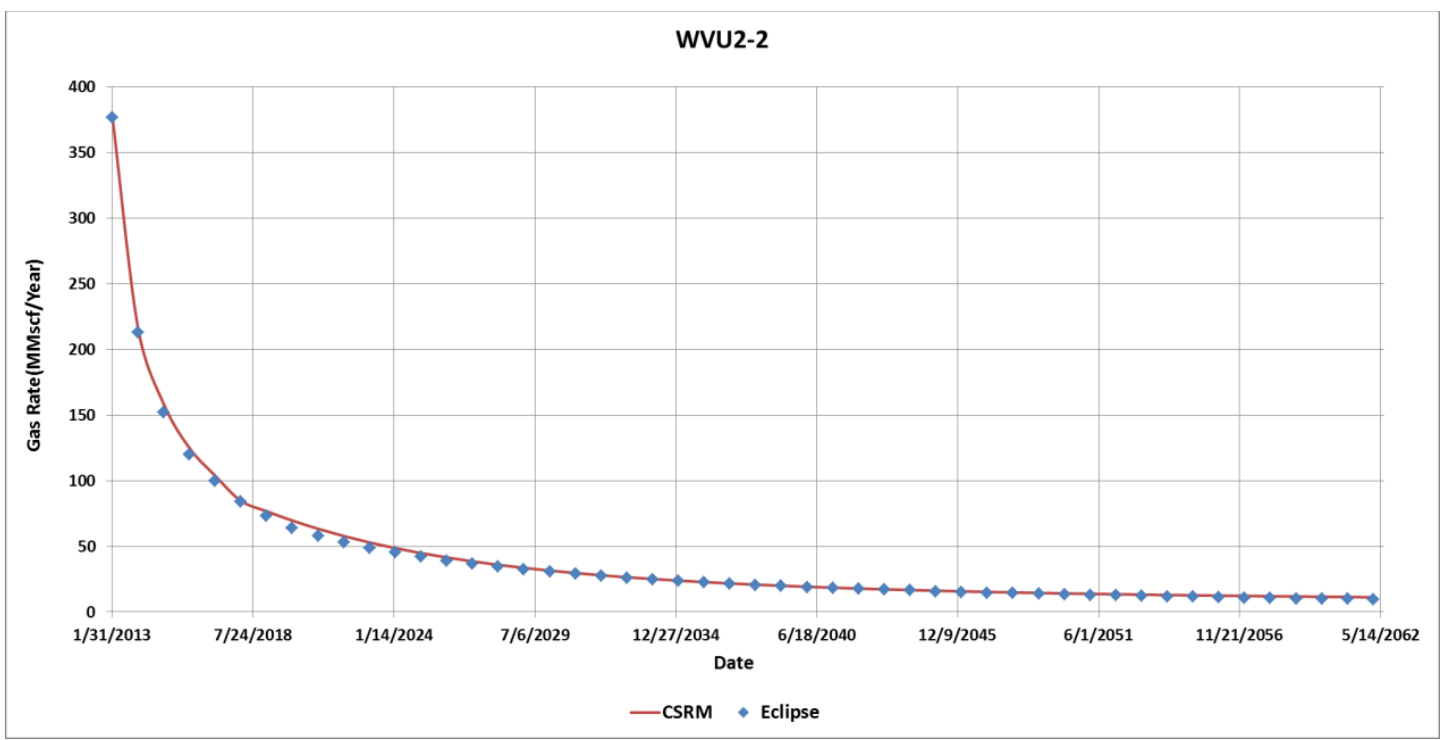

Figure 4-20. Combined monthly and annual proxies- Annual gas rate production WVU-2-2 lateral

\subsection{Data-Driven Proxy Models Validation by Blind Case}

During Data-driven proxy model development, some of the data were not included in the training set and used for calibration and verification purpose. Nevertheless, in order to test the predictive capability of the developed shale proxy models and taking validation one-step further, a simulation run is designed in a 
way to be completely different (but in uncertainty range) from those previous runs, which were used for developing proxy models.

Figure 4-21 shows the predictive capability of the developed Data-driven proxy models .In this figure the blind simulated gas production rates (Blue dots) for different time resolutions of daily, monthly and annually (from top to bottom) are compared with the shale proxy models (Red lines) for WVU1 lateral. Good results show that the developed Data-driven shale proxy model can be effectively used as a tool for uncertainty quantification since thousands of shale surrogate model runs can be made in a few seconds to fulfill the requirement of the Monte Carlo simulation for uncertainty analysis.

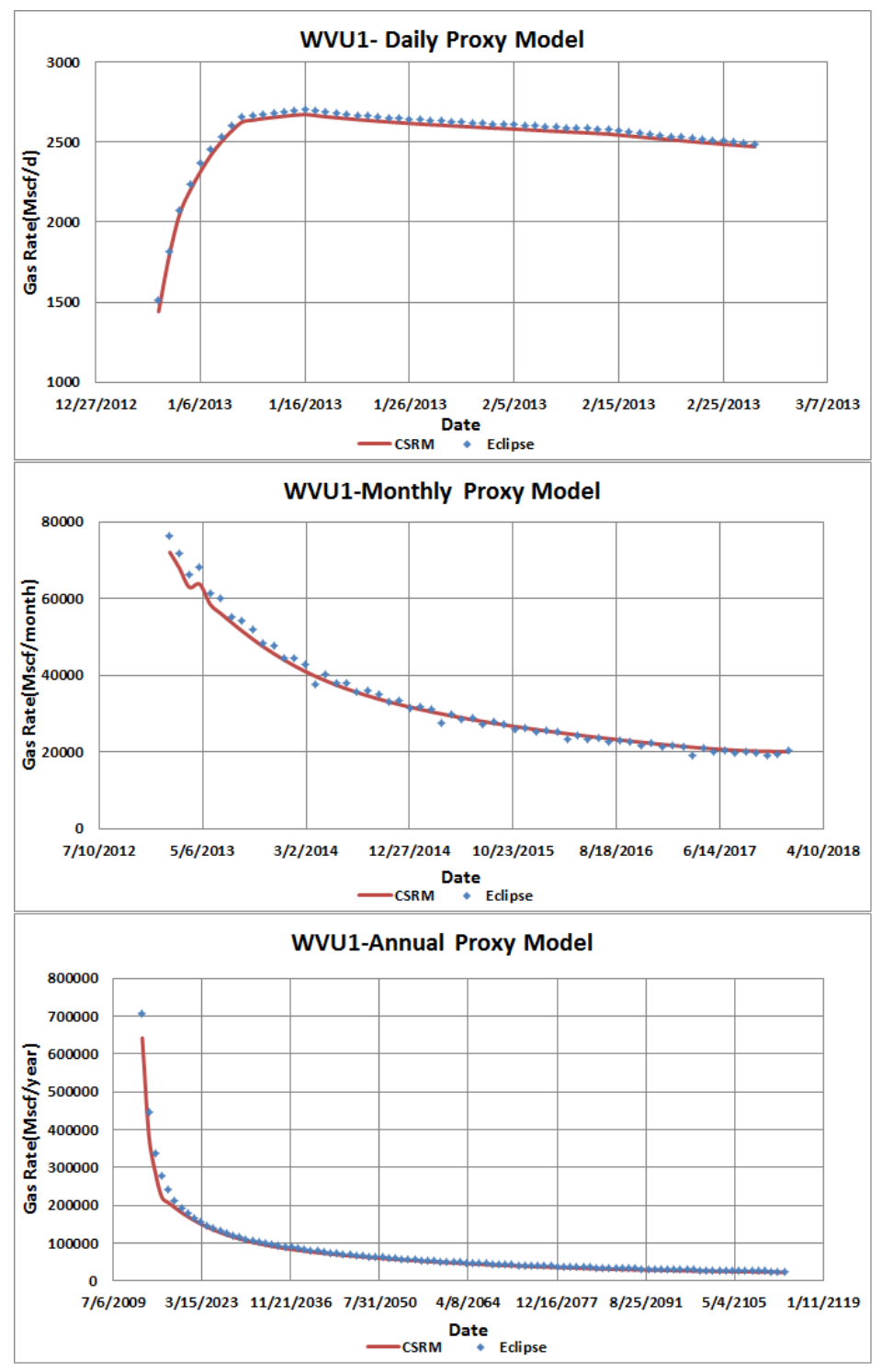

Figure 4-21. Data-driven proxy models validation using blind run for WVU1 for different time resolutions of daily, monthly and annually( from top to bottom) 


\subsection{Summary and Conclusions}

In this chapter, a Next-generation shale proxy model based on pattern recognition capabilities of Artificial Intelligence is introduced to assist in shale gas modeling and simulation in the case of using EHF technique.

Data-driven proxy models are developed for three different time resolutions of daily, monthly and annually that are able to generate production profile for each cluster with acceptable accuracy. This technique enables the shale modelers to use all functionality of the developed simulation model and predict shale gas production behavior at each cluster faster with high accuracy compared with the numerical simulator.

Moreover, it can be used as an assisted history-matching tool and also for uncertainty analysis and designing new wells with different clusters of hydraulic fracture. Because the modeling is performed at the HF cluster level, the Data-drive proxy model is robust enough to predict the new well production profile with any definite number of hydraulic fractures and any assigned properties in uncertainty domain. 


\section{CHAPTER 5 -TECHNICAL ASPECTS OF $\mathrm{CO}_{2}$-ENHANCED GAS RECOVERY AND STORAGE $\left(\mathrm{CO}_{2}\right.$-EGR\&S) IN DEPLETED SHALERESERVOIR}

\subsection{Summary}

The objective of this chapter is to evaluate $\mathrm{CO}_{2}$ injection process for the purpose of production enhancement and $\mathrm{CO}_{2}$ sequestration in the Marcellus shale gas reservoir using numerical simulation. All simulation runs are based on the depleted, history matched numerical reservoir simulation model that is already developed and is explained in previous chapters. By designing different injection scenarios, the best practices to enhance methane recovery, minimize $\mathrm{CO}_{2}$ production while maximizing the amount of stored $\mathrm{CO}_{2}$ in shale formation are proposed.

\subsection{Methodology}

The workflow for evaluating $\mathrm{CO}_{2}$ Enhance Gas Recovery and Storage $\left(\mathrm{CO}_{2}\right.$-EGR\&S) in this study is summarized in Figure 5-1.Depleting the history-matched model to the point that makes the $\mathrm{CO}_{2}$ process feasible is the first step in this workflow that is needed to be determined. Different injection scenarios are defined based on a series of modifications in all involved parameters. The parameters that will be modified in each scenario are Well patterns (injector and producer spacing), Reservoir characteristics, Hydraulic fracture properties, Sorption features (Langmuir isotherms, sorption time and diffusion coefficient), and Operational constraints (bottom-hole injection pressure, duration of injection). Finally, the best injection practice is proposed.

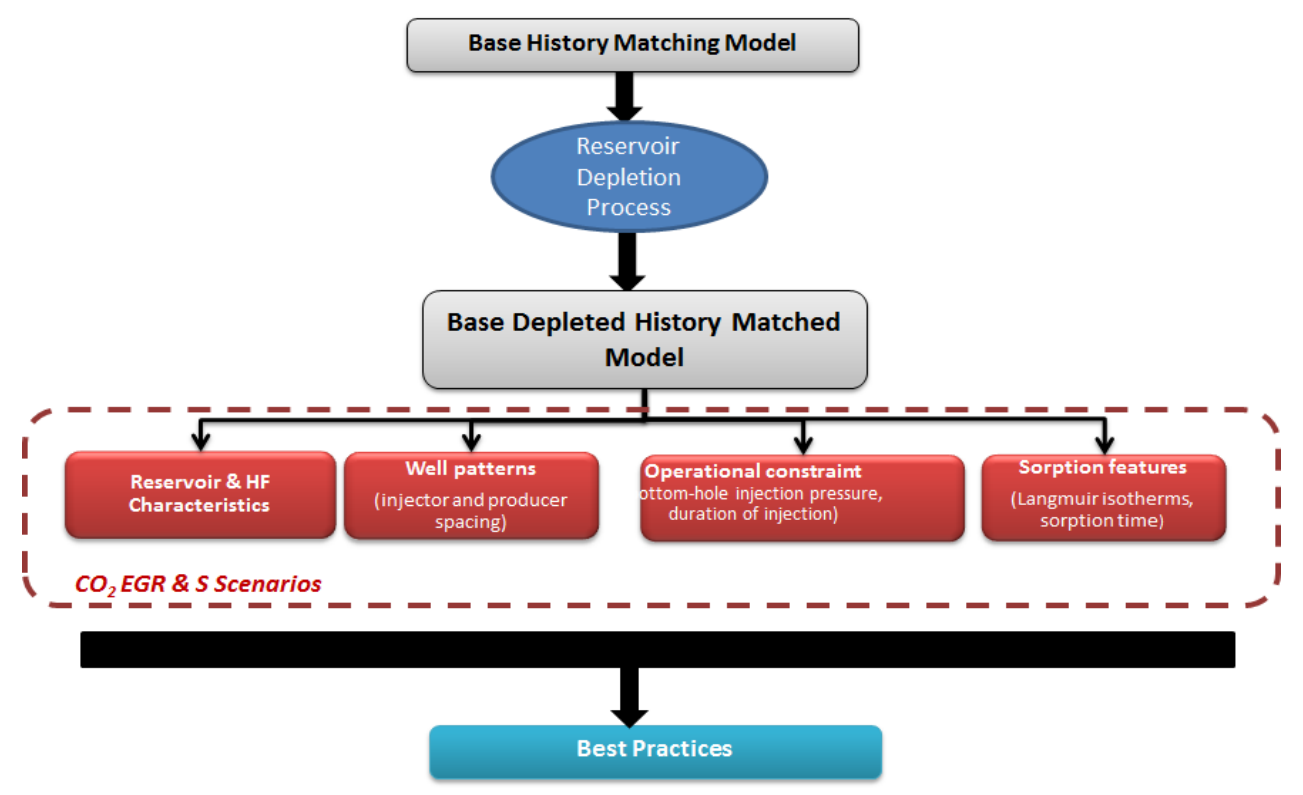

Figure 5-1. CO2-Enhanced Gas Recovery and Storage Workflow 


\section{3 $\mathrm{CO}_{2}$ Injection/Storage Scenarios}

Part of the history matched WVU pad with five laterals is used for performing injection practices. As shown in Figure 5-2 the distances between horizontal laterals in WVU pad are between 400 to 2,094 ft.

Figure 5-3 shows the distribution of distance between laterals in the shale asset that is the subject of this study. Comparing the distances between the laterals in WVU pad with the bar chart (Figure 5-3) demonstrates that this pad can be a good representative of part of Marcellus shale asset in SW PA in terms of well configuration as well as reservoir and hydraulic fracture characteristics that has already been discussed in previous chapters. Orange dotted-line boxes in Figure 5-3 represent the range of inside distances between the WVU pad laterals.

The first step in the process of modeling $\mathrm{CO}_{2}$ injection is to evaluate a range of injection scenarios and then, based on the results of that modeling effort; propose an appropriate set of injection cases. The most appropriate injection scenario attempts to maximize methane production while delays $\mathrm{CO}_{2}$ breakthrough time (BT) and maximizing the amount of $\mathrm{CO}_{2}$ that is stored.

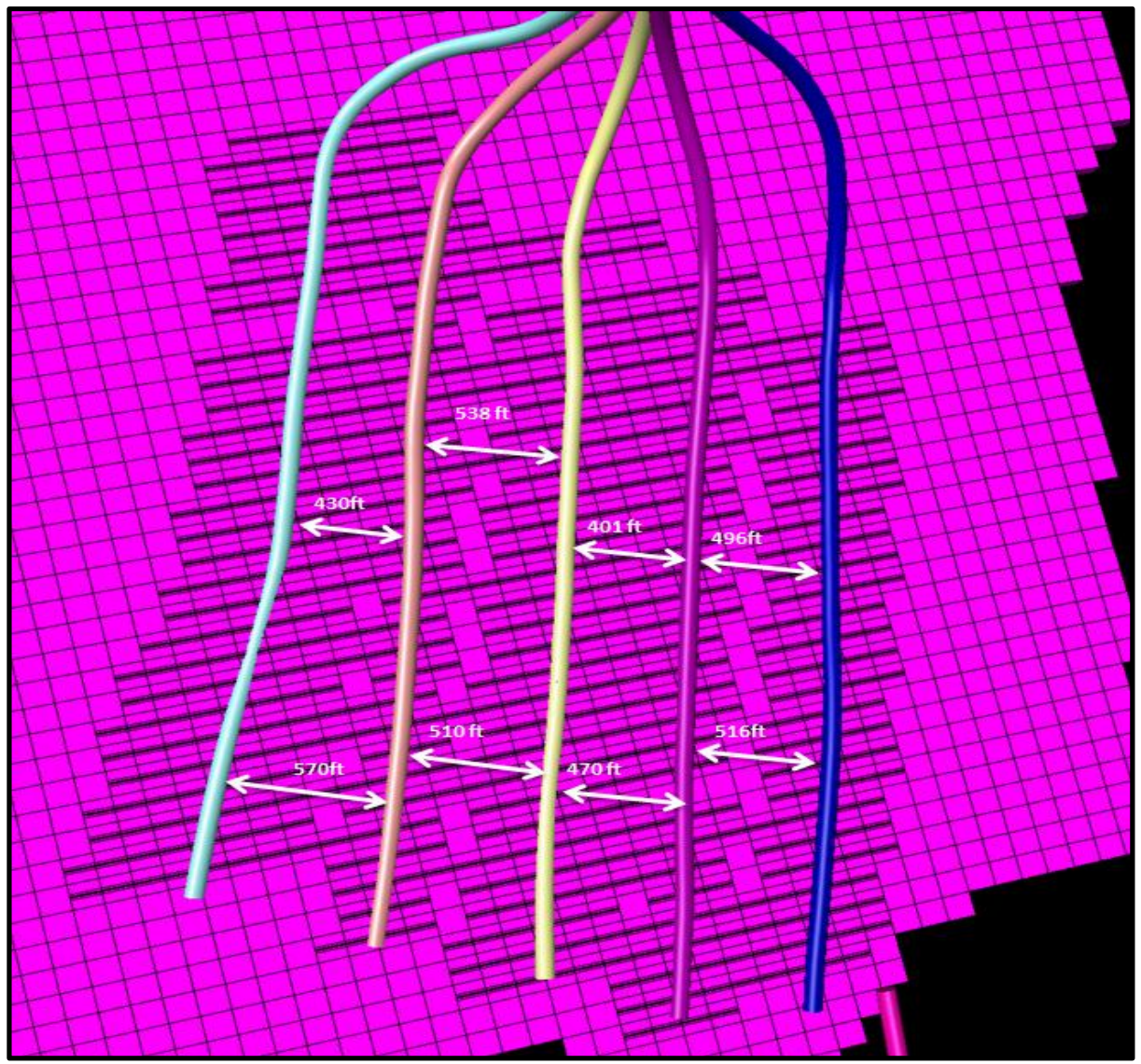

Figure 5-2.Highlighting Distance between adjacent laterals in part of WVU pad 


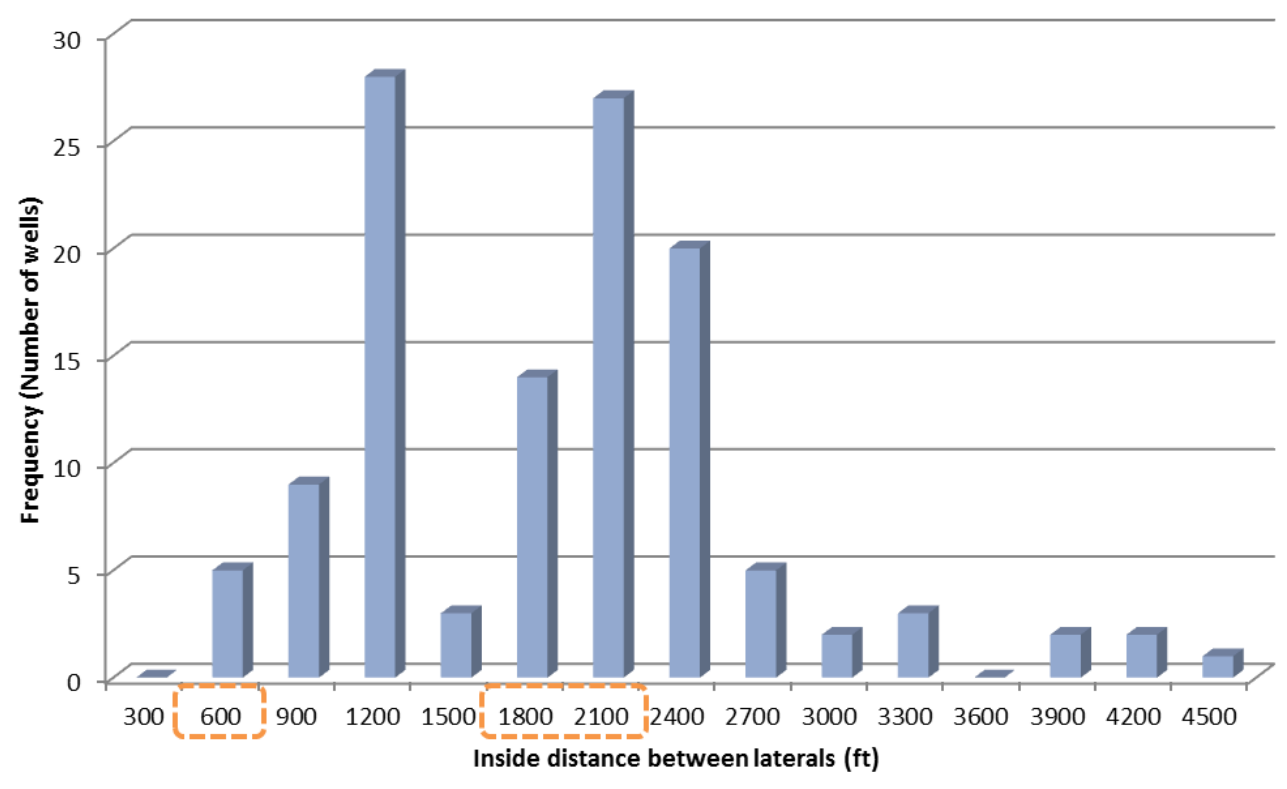

Figure 5-3. Distribution of distances between lateral for Marcellus shale asset (Total number of 135 laterals)

With this strategy in mind, it is impractical to inject $\mathrm{CO}_{2}$ into all the laterals. Instead, the proper set of injector-producer laterals must be selected with the aim of enhancing methane production from the offset well(s). This is due to the fact that communication between hydraulic fractures in the so-called SRV (Stimulated Reservoir Volume) regions contributes to early breakthrough.

The injection strategy includes five cases with different distances between injection and production well(s). Figure 5-4 illustrates these five cases and shows the minimum and maximum distances between the producer(s)/injector pairs. Moreover, the number of clusters for each case and for each well is shown in this figure.

Before starting the injection process, all the wells in each of the cases are put on production for 100 years, which is called base injection case. When one of the production wells, which is going to be converted to an injection well, produced $75 \%$ of its accessible gas, the $\mathrm{CO}_{2}$ injection starts and continues until the end of the $\mathrm{t}=100$ years. This usually happens between 45 to 55 years after the start of production.

It should be noted that in all cases, WVU2-1 remains as a production well, while the injector is changing. Therefore, not only the distance between the laterals is changing, but also reservoir characteristics and hydraulic fracture properties for each cluster in production and prospective injection wells are changing as well. 


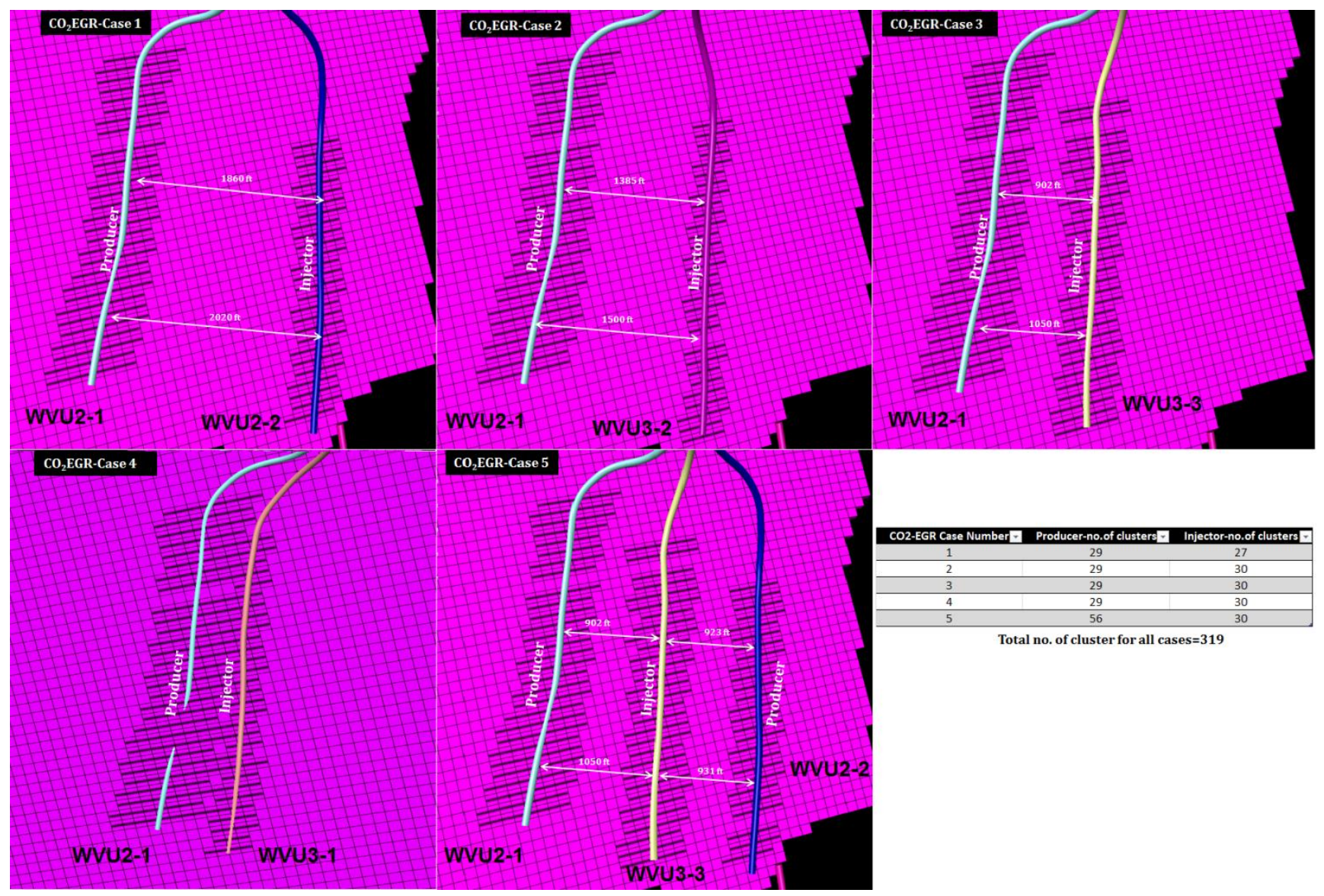

Figure 5-4. Schematics of five different injection scenarios for CO2 -EGR \&S process

\subsection{1 $\mathrm{CO}_{2}$-EGR\&S- Scenario 1}

In scenario 1, WVU2-1 is defined as the production well that is located around $2000 \mathrm{ft}$. away from WVU2-2, which is the injector. The production well has 29 clusters of hydraulic fractures with fracture conductivity from 2.06 to $5.29 \mathrm{md}-\mathrm{ft}$, while the injector has 27 clusters with fracture conductivity of 1.23 to 4.39 md-ft.

As was mentioned before, for the base injection case, both wells (producer and prospective injector) have been produced for 100 years. Before starting injection process, both wells that are already history matched are continuing their production to reach to the point that the WVU2-2 produces $75 \%$ of its accessible gas (

Table 5-1). According to this table, the prospective injection well (WVU2-2) is produced $75 \%$ of its accessible reserve after 40.5 years of production and the well block pressure is declined from initial reservoir pressure of 3890 psi to 480 psi. At that point, $\mathrm{CO}_{2}$ injection is started at constant bottomhole 
injection pressure (as a constraint) and the simulation output is $\mathrm{CO}_{2}$ injection rate and $\mathrm{CO}_{2}$ and $\mathrm{CH}_{4}$ production rates.

Table 5-1.Base injection case for scenario1

\begin{tabular}{cccccc}
\hline Well name & $\begin{array}{c}\text { Gas rate @ the } \\
\text { end of 100 } \\
\text { years(Mscf/d) }\end{array}$ & $\begin{array}{c}\text { 100 years } \\
\text { Cum. gas } \\
\text { production } \\
\text { (Bcf) }\end{array}$ & $\begin{array}{c}\text { 75\% of } \\
\text { Reachable } \\
\text { Reserve (Bcf) }\end{array}$ & $\begin{array}{c}\text { Time to reach } \\
\mathbf{7 5 \%} \text { of } \\
\text { accessible } \\
\text { reserve(year) }\end{array}$ & $\begin{array}{c}\text { P_block after } \\
\text { 100 years(psi) }\end{array}$ \\
\hline WVU2-1 & 127.0 & 11.072 & 8.305 & 51.5 & 523 \\
WVU2-2 & 50.9 & 6.781 & 5.086 & 40.5 & 480 \\
\hline
\end{tabular}

The total cumulative gas production for both well after 100 years of production is around $17.85 \mathrm{Bcf}$. The best injection case is the one to yield at least the same recovery as the base case provides over 100 years and store a considerable amount of $\mathrm{CO}_{2}$. For that reason, selecting an appropriate bottomhole injection pressure plays significant role in attaining the objective of $\mathrm{CO}_{2}$ enhanced gas recovery and storage process, which is maximizing methane recovery with minimum carbon dioxide breakthrough, while maximizing the amount of injected $\mathrm{CO}_{2}$ in the formation.

By setting the bottomhole injection pressure at very low value, the injection will be stopped after a short period because of an increase in injector's well block pressure beyond the constant bottomhole injection pressure value.

On the other hand, injection at very high pressure may create additional fracture in the formation and also may expedite the $\mathrm{CO}_{2}$ breakthrough time. Therefore, $50 \%$ lithostatic pressure (3360 psi in this study) is set to maintain a healthy factor of safety to ensure that fracture propagation is avoided and containment remains intact.

For scenario 1, two cases are defined based on different bottomhole injection pressures. $\mathrm{CO}_{2}$ is injected at $25 \%$ ( Case 1 ) and 50\% (Case2) of lithostatic pressure.

Table 5-2 represents the results of $\mathrm{CO}_{2}$ injection in case 1. According to this table, $6.26 \mathrm{E}+04$ tons of $\mathrm{CO}_{2}$ are injected at a constant bottomhole pressure of 1680 (25\% of lithostatic pressure) for 59.5 years with zero $\mathrm{CO}_{2}$ breakthrough. Therefore, all injected $\mathrm{CO}_{2}$ is stored in the reservoir and methane production from WVU2-1 is enhanced by $1.15 \%$. 
Very low production enhancement is expected because of the large distance between the production and injection laterals. The total production from WVU2-1 and WVU2-2 is $16.286 \mathrm{Bcf}$, which is lower than the base case scenario.

Table 5-2-Injection scenario 1-Case 1: Duration of injection and the amount of recovered $\mathrm{CH} 4$ and stored $\mathrm{CO} 2$

\begin{tabular}{|c|c|c|c|c|c|c|c|c|}
\hline $\begin{array}{l}\text { Well } \\
\text { name }\end{array}$ & $\begin{array}{l}\text { Well } \\
\text { type }\end{array}$ & $\begin{array}{c}\text { Injection } \\
\text { starts @ } \\
\mathbf{t}=(\text { years })\end{array}$ & $\begin{array}{c}\text { Injection } \\
\text { period } \\
\text { (years) }\end{array}$ & $\begin{array}{c}\text { Injection } \\
\text { pressure } \\
\text { (psi) }\end{array}$ & $\begin{array}{c}\mathrm{CH}_{4} \mathrm{Cum} . \\
\text { production } \\
\text { (Bcf) }\end{array}$ & $\begin{array}{l}\mathrm{CO}_{2} \mathrm{Cum} . \\
\text { production } \\
\text { (Bcf) }\end{array}$ & $\begin{array}{c}\text { Cum. } \\
\text { Injected } \\
\mathrm{CO}_{2} \\
\text { (ton) } \\
\end{array}$ & $\begin{array}{c}\text { Total } \\
\text { Stored } \\
\mathrm{CO}_{2} \\
\text { (ton) } \\
\end{array}$ \\
\hline WVU2-1 & Prod. & ------ & --------- & ------- & 11.2 & 0.0 & ---- & \\
\hline WVU2-2 & Inj. & 40.5 & 59.5 & 1680 & 5.086 & --- & $6.26 \mathrm{E}+04$ & $6.26 \mathrm{E}+04$ \\
\hline
\end{tabular}

In the second case, $\mathrm{CO}_{2}$ is injected at $3360 \mathrm{psi}$ (50\% of lithostatic pressure) for 59.5 years. The outcome is summarized in Table 5-3.Methane production is enhanced by 3.1\% in WVU2-1 and 3.27E+05 tons of $\mathrm{CO}_{2}$ are injected and completely stored in the formation without any trace of $\mathrm{CO}_{2}$ breakthrough in the producing offset lateral. The total production from WVU2-1 and WVU2-2 is 16.513 Bcf, which is still below the total methane production in the base case.

By comparing the total amount of injected $\mathrm{CO}_{2}$ in Case 1 and Case2, it can be observed that the doubling of the bottomhole injection pressure gives a 5.2 times increase in the total amount of injected $\mathrm{CO}_{2}$. Therefore, there is not a linear relationship between the injection pressure and cumulative injected $\mathrm{CO}_{2}$.

Table 5-3-Injection scenario 1-Case 2: Duration of injection and the amount of recovered $\mathrm{CH} 4$ and stored $\mathrm{CO} 2$

\begin{tabular}{ccccccccc}
\hline $\begin{array}{c}\text { Well } \\
\text { name }\end{array}$ & $\begin{array}{c}\text { Well } \\
\text { type }\end{array}$ & $\begin{array}{c}\text { Injection } \\
\text { starts } \mathbf{a} \\
\mathbf{t =} \text { (years) }\end{array}$ & $\begin{array}{c}\text { Injection } \\
\text { period } \\
\text { (years) }\end{array}$ & $\begin{array}{c}\text { Injection } \\
\text { pressure } \\
\text { (psi) }\end{array}$ & $\begin{array}{c}\mathbf{C H}_{4} \text { Cum. } \\
\text { production } \\
\text { (Bcf) }\end{array}$ & $\begin{array}{c}\mathbf{C O}_{2} \text { Cum. } \\
\text { production } \\
\text { (Bcf) }\end{array}$ & $\begin{array}{c}\text { Cum. } \\
\text { Injected } \\
\mathbf{C O}_{2} \text { (ton) }\end{array}$ & $\begin{array}{c}\text { Total } \\
\text { Stored } \\
\mathbf{C O}_{2} \text { (ton) }\end{array}$ \\
\hline WVU2-1 & Prod. & ----- & ------ & ----- & 11.427 & 0.0 & ---- & \\
WVU2-2 & Inj. & 40.5 & 59.5 & 3360 & 5.086 & --- & $3.27 \mathrm{E}+05$ & $3.27 \mathrm{E}+05$ \\
\hline
\end{tabular}

Figure 5-5 shows the $\mathrm{CO}_{2}$ injection rate and compares the cumulative methane production before and after $\mathrm{CO}_{2}$ injection in Case2. The cum. $\mathrm{CH}_{4}$ production is started to increase after 25-30 years of injection that confirms a very slow counter diffusion process in the system. In addition, $\mathrm{CO}_{2}$ injection rate declines from $1650 \mathrm{Mscf} /$ day to around $160 \mathrm{Mscf} /$ day after 50 years of injection. At the beginning of the injection, $\mathrm{CO}_{2}$ occupies the fracture and pore spaces quickly and due to the fact that $\mathrm{CO}_{2}$ plume cannot extend above and beyond the so-called SRV region, together with the slow counter diffusion process, the 
pressurized $\mathrm{CO}_{2}$ region around the injector does not have the capability to accept more $\mathrm{CO}_{2}$ at high rate. Therefore, a sharp decline in the $\mathrm{CO}_{2}$ injection rate is observed.

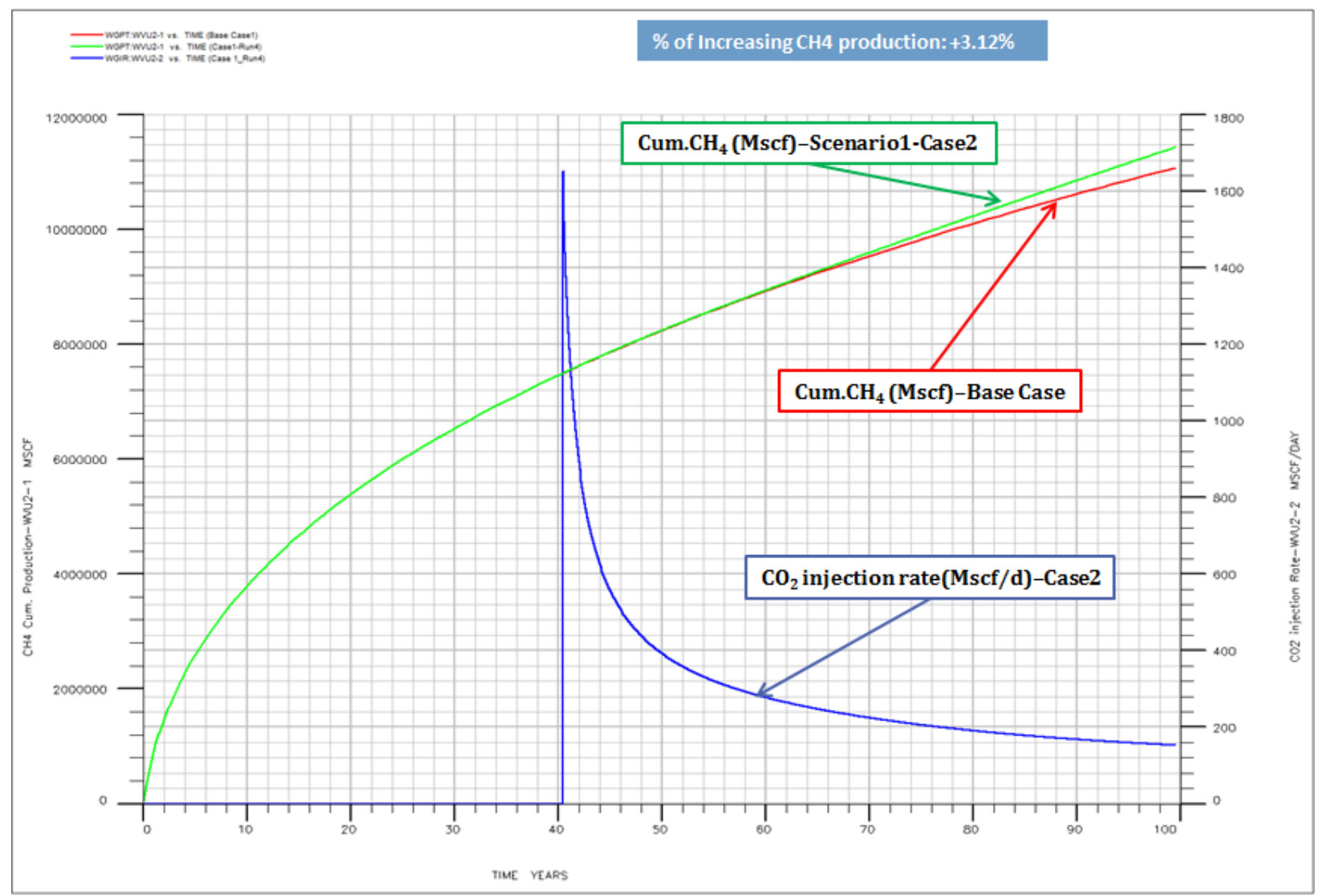

Figure 5-5. $\mathrm{CO} 2$ injection rate profile and Comparison of $\mathrm{CH} 4$ cumulative production in base case and Case 2

In this graph, red and green solid lines show the cum. $\mathrm{CH}_{4}$ production in the base case and Case2, respectively; while the blue solid line represents the $\mathrm{CO}_{2}$ injection rate at a constant bottomhole injection pressure of 3360 psi.

In both cases, three inactive wells between producer and injector are used as monitoring wells to monitor the pressure and molar density $\left(\mathrm{CH}_{4}\right.$ and $\left.\mathrm{CO}_{2}\right)$ changes as a function of time and space in the system.

Figure 5-6 and Figure 5-7illustrate the molar density $\left(\mathrm{CH}_{4}\right.$ and $\left.\mathrm{CO}_{2}\right)$ changes in the monitoring wells (WVU3-1, WVU3-3 \& WVU3-2). In these figures, blue solid lines show the molar density changes for WVU3-2 as a monitoring well, which is the closest offset well to the injection well (WVU2-2), while the green solid lines belong to the closest offset well (WVU3-1) to the production well (WVU2-1). The red solid line is the monitoring well WVU3-3, which is the middle lateral in WVU pad. 
As shown in Figure 5-6, the molar density of methane is decreasing during the production. (History matching and depletion periods).The increasing and also plateau trends in methane molar density in

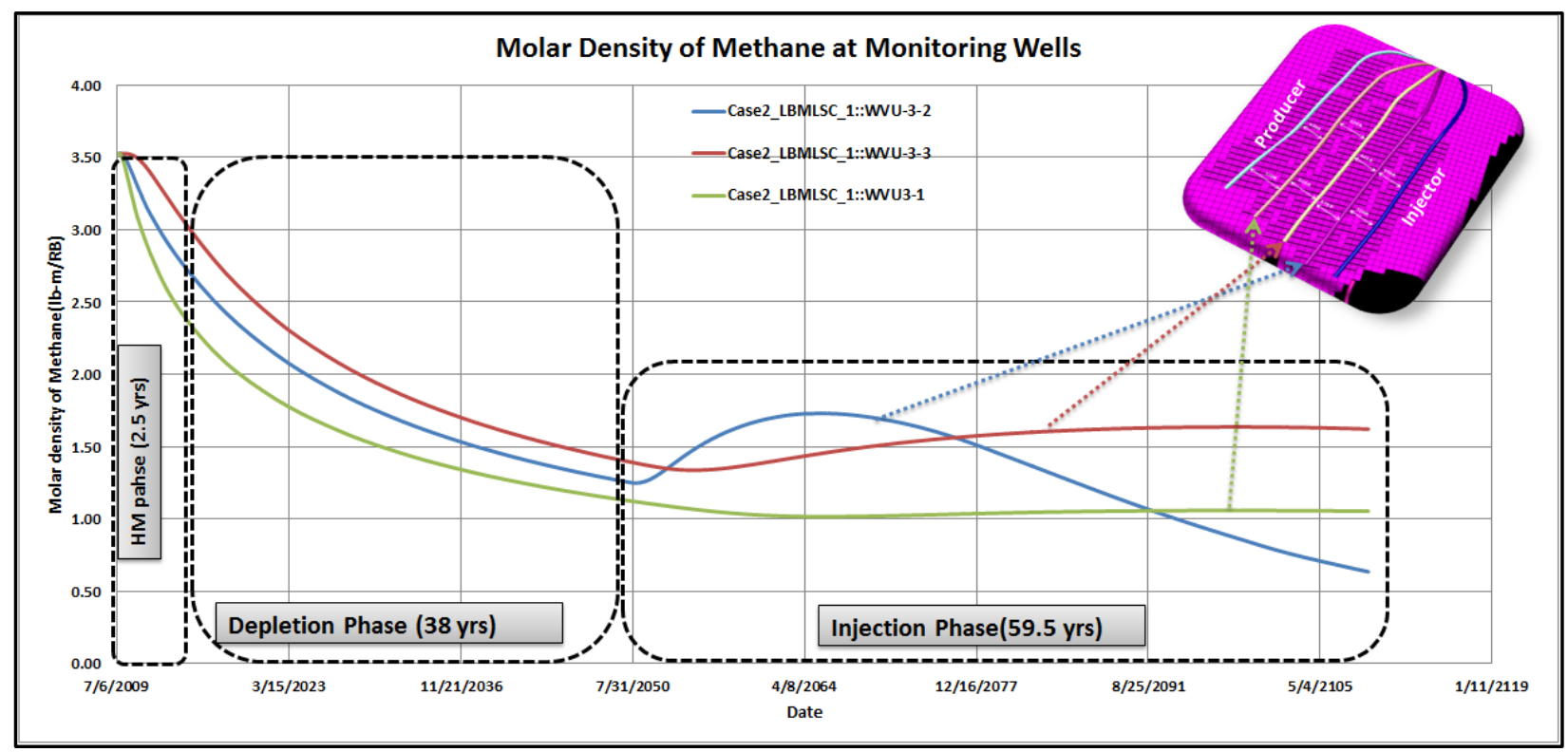

Figure 5-6. Methane molar density changes in monitoring wells in the scenario1- Case2

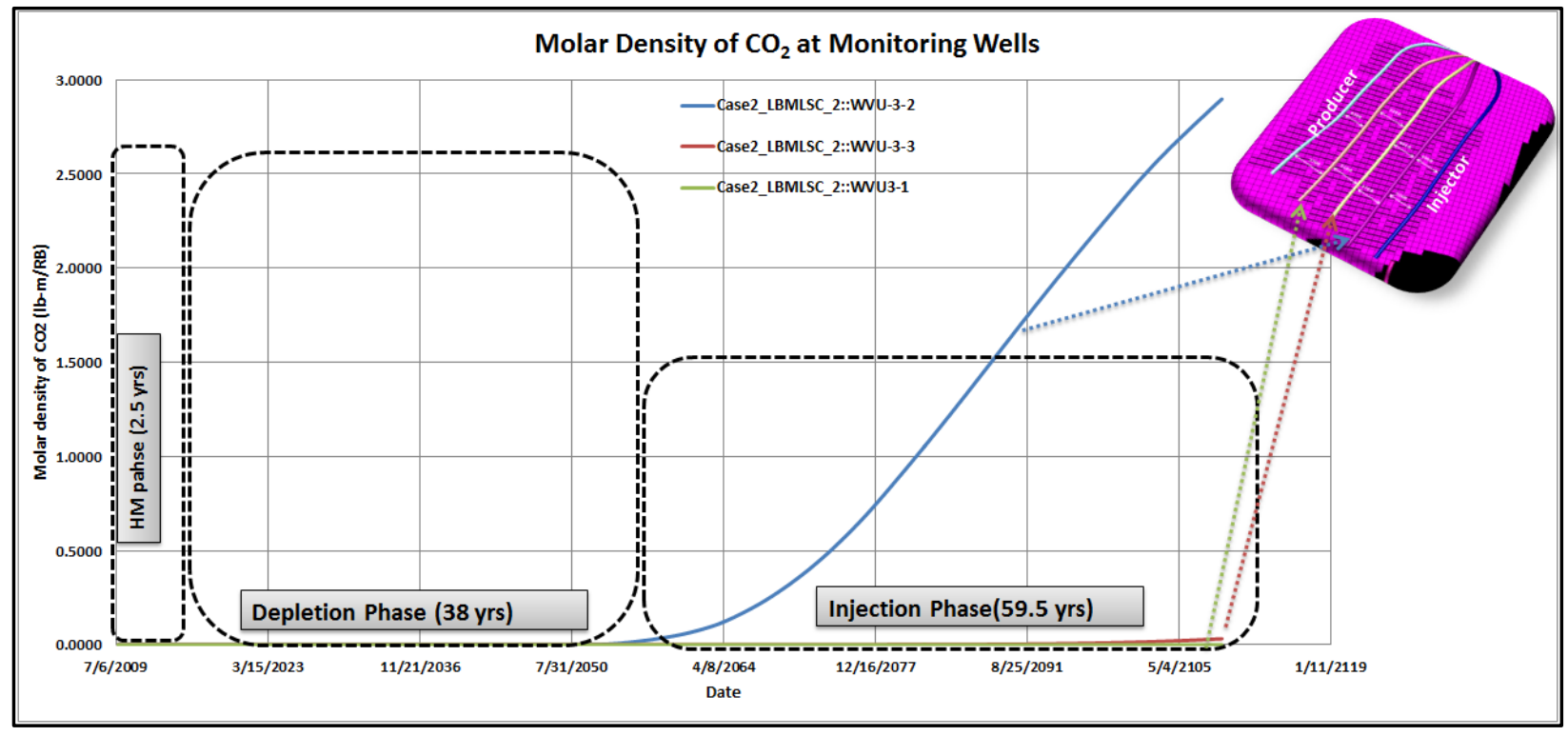

Figure 5-7.Carbon dioxide molar density changes in monitoring wells in the scenario1- Case2

monitoring wells (WVU3-3 and WVU3-1 relatively), are observed as a result of counter-diffusion process and replacement of the methane with carbon dioxide in shale matrix. Since the diffusion of $\mathrm{CH}_{4}$ in nanopermeability pores is very slow, therefore the farthest well is less impacted in this process (WVU3-1). 
On the other hand, an increase in methane molar density, in the closest monitoring well to the injector, followed by sharp decreases in molar density profile is observed (Blue line in Figure 5-6). The reason for this behavior is that, when the $\mathrm{CO}_{2}$ occupied the fractures, because still inorganic pores are filled with desorbed methane, therefore $\mathrm{CO}_{2}$ may not be able to contact the shale fabric to start the displacement process. In this situation, $\mathrm{CO}_{2}$ pushing the methane and due to higher concentration of $\mathrm{CH}_{4}$ in the inorganic pores compare with the organic ones, the re-adsorption of methane is happening.

As a result, a hump in the methane molar density profile of WVU3-2 is seen. Having lower free methane in the pores following with an increase in the methane molar density in the organic pores due to readsorption, make the $\mathrm{CO}_{2}$ molecules to be sufficiently in contact with the organic pores and therefore the $\mathrm{CO}_{2}$ replaces methane, due to higher affinity, and a fast decline in $\mathrm{CH}_{4}$ molar density is happening.

Figure 5-7 shows the changes in the molar density of $\mathrm{CO}_{2}$ in the monitoring well. In this graph, the molar density of $\mathrm{CO}_{2}$ in the closet monitoring well to the injector increases dramatically as a result of counter diffusion and replacement of $\mathrm{CH}_{4}$ by $\mathrm{CO}_{2}$. This increase in carbon dioxide molar density is very slow in the other monitoring wells due to the very slow diffusion of $\mathrm{CO}_{2}$ in the matrix.

The well block pressure changes during the history matching and depletion period and also during the injection are shown in Figure 5-8. The blue line shows the injector's block pressure and the black solid line illustrates the block pressure changes in the production well. The $\mathrm{P}_{\text {block }}$ for the monitoring wells are shown with purple, green and red solid lines, according to their distances from the injector (From closest to farthest monitoring well). The maximum pressure increase is happening in injection well, whereas a small elevation in block pressure can be observed in the production well. 


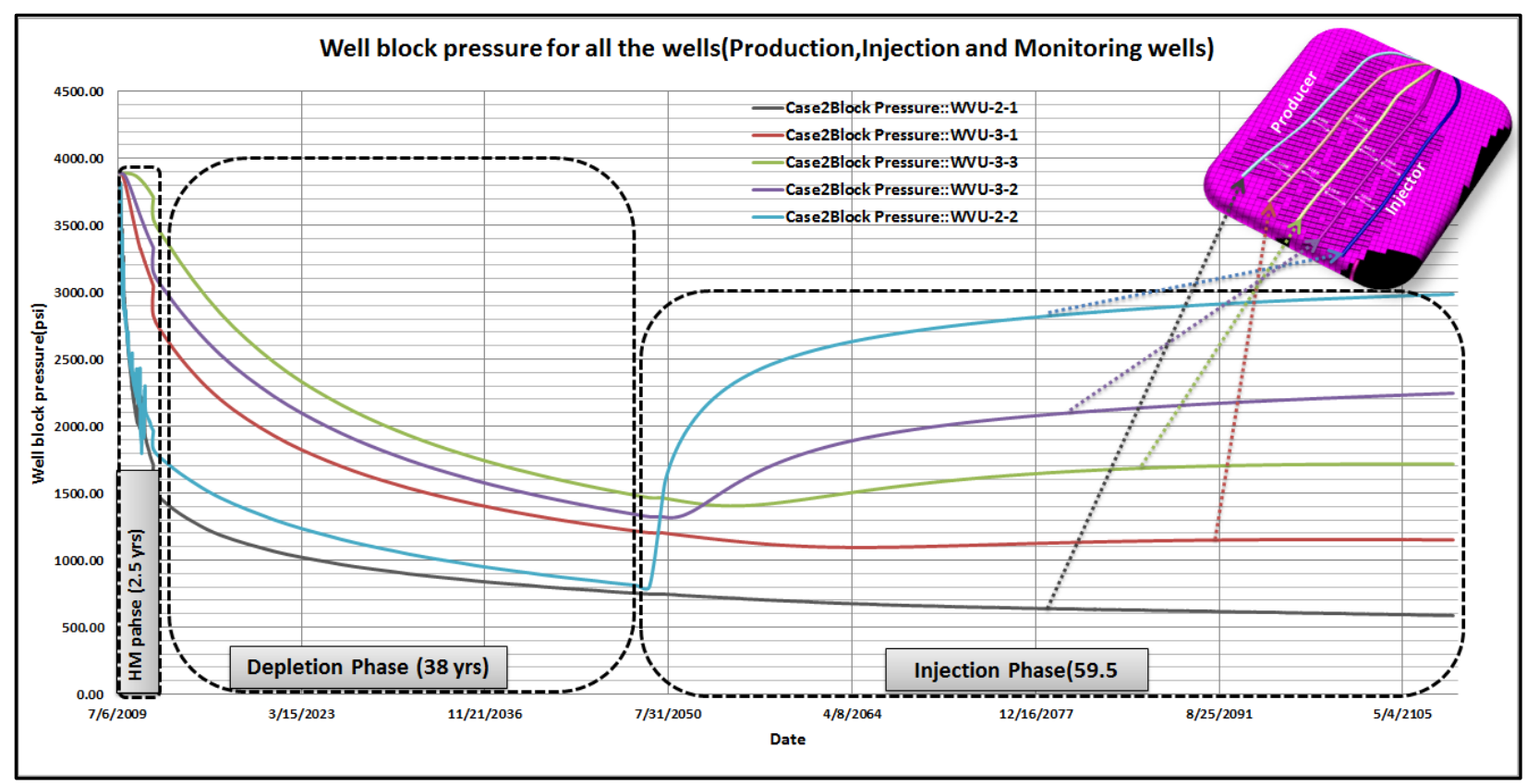

Figure 5-8. Well block pressure profile for all the wells during history matching, depletion and injection

\subsection{2 $\mathrm{CO}_{2}$-EGR\&S- Scenario 2}

In the second scenario, WVU2-1 stays as the production well, which is, located around $1400 \mathrm{ft}$. away from WVU3-2 that is the injector and has 30 clusters with fracture conductivity of 0.56 to $4.94 \mathrm{md}-\mathrm{ft}$.

Table 5-4 summarizes the base case information for scenario 2. According to this table, after 42.5 years of production, the prospective injection well (WVU3-2) is produced $75 \%$ of its accessible reserve and the well block pressure is declined from initial reservoir pressure of 3890 psi to 371 psi.

Table 5-4.Base injection case for scenario2

\begin{tabular}{cccccc}
\hline Well name & $\begin{array}{c}\text { Gas rate @ the } \\
\text { end of 100 } \\
\text { years(Mscf/d) }\end{array}$ & $\begin{array}{c}\text { 100 years } \\
\text { Cum. gas } \\
\text { production } \\
\text { (Bcf) }\end{array}$ & $\begin{array}{c}\mathbf{7 5 \%} \text { of } \\
\text { Reachable } \\
\text { Reserve (Bcf) }\end{array}$ & $\begin{array}{c}\text { Time to reach } \\
\mathbf{7 5 \%} \text { of } \\
\text { accessible } \\
\text { reserve(year) }\end{array}$ & $\begin{array}{c}\text { P_block after } \\
\text { 100 years(psi) }\end{array}$ \\
\hline WVU2-1 & 116.4 & 10.22 & 7.66 & 50.5 & 501 \\
WVU3-2 & 63.4 & 7.79 & 5.84 & 42.5 & 371 \\
\hline
\end{tabular}

In this scenario, two cases are defined based on different bottomhole injection pressure. $\mathrm{CO}_{2}$ is injected at $25 \%$ ( Case 1) and 50\% (Case2) of lithostatic pressure. 
Table 5-5 summarizes the results of $\mathrm{CO}_{2}$ injection in Case 1. According to this table, $8.37 \mathrm{E}+04$ tons of $\mathrm{CO}_{2}$ are injected at a constant bottomhole pressure of 1680 (25\% of lithostatic pressure) for 57.5 years with zero $\mathrm{CO}_{2}$ breakthrough. Therefore, all injected $\mathrm{CO}_{2}$ is stored in the reservoir and methane production from WVU2-1 is enhanced by $2.64 \%$. Because of lower distance between the production and injection well, slight increase in production is achieved in this scenario compares to the scenario1. Nevertheless, the total methane production from WVU2-1 and WVU3-2 is $16.33 \mathrm{Bcf}$, which is still lower than the total production of $18.01 \mathrm{Bcf}$ in the senario2-base case.

Table 5-5. Injection scenario 2-Case 1: Duration of injection and the amount of recovered $\mathrm{CH} 4$ and stored $\mathrm{CO} 2$

\begin{tabular}{|c|c|c|c|c|c|c|c|c|}
\hline $\begin{array}{c}\text { Well } \\
\text { name }\end{array}$ & $\begin{array}{l}\text { Well } \\
\text { type }\end{array}$ & $\begin{array}{c}\text { Injection } \\
\text { starts @ } \\
\mathbf{t}=\text { (years) }\end{array}$ & $\begin{array}{c}\text { Injection } \\
\text { period } \\
\text { (years) }\end{array}$ & $\begin{array}{l}\text { Injection } \\
\text { pressure } \\
\quad \text { (psi) }\end{array}$ & $\begin{array}{c}\mathrm{CH}_{4} \mathrm{Cum} \text {. } \\
\text { production } \\
\text { (Bcf) }\end{array}$ & $\begin{array}{c}\mathrm{CO}_{2} \mathrm{Cum} . \\
\text { production } \\
\text { (Bcf) }\end{array}$ & $\begin{array}{c}\text { Cum. } \\
\text { Injected } \\
\mathrm{CO}_{2} \\
\text { (ton) }\end{array}$ & $\begin{array}{c}\text { Total } \\
\text { Stored } \\
\mathrm{CO}_{2}(\text { ton })\end{array}$ \\
\hline WVU2-1 & Prod. & ------ & -------- & ------- & 10.49 & 0.0 & ---- & \\
\hline WVU3-2 & Inj. & 42.5 & 57.5 & 1680 & 5.84 & --- & $8.37 \mathrm{E}+04$ & $8.37 \mathrm{E}+04$ \\
\hline
\end{tabular}

Table 5-6. Injection scenario 2-Case 2: Duration of injection and the amount of recovered $\mathrm{CH} 4$ and stored $\mathrm{CO} 2$

\begin{tabular}{|c|c|c|c|c|c|c|c|c|}
\hline $\begin{array}{c}\text { Well } \\
\text { name }\end{array}$ & $\begin{array}{l}\text { Well } \\
\text { type }\end{array}$ & $\begin{array}{c}\text { Injection } \\
\text { starts @ } \\
\mathrm{t}=\text { (years) }\end{array}$ & $\begin{array}{c}\text { Injection } \\
\text { period } \\
\text { (years) }\end{array}$ & $\begin{array}{c}\text { Injection } \\
\text { pressure } \\
\text { (psi) }\end{array}$ & $\begin{array}{c}\mathrm{CH}_{4} \mathrm{Cum} \text {. } \\
\text { production } \\
\text { (Bcf) }\end{array}$ & $\begin{array}{c}\mathrm{CO}_{2} \mathrm{Cum} \text {. } \\
\text { production } \\
\text { (Bcf) }\end{array}$ & $\begin{array}{c}\text { Cum. } \\
\text { Injected } \\
\mathrm{CO}_{2} \text { (ton) } \\
\end{array}$ & $\begin{array}{c}\text { Total } \\
\text { Stored } \\
\mathrm{CO}_{2} \text { (ton) } \\
\end{array}$ \\
\hline WVU2-1 & Prod. & ------ & -------- & ------- & 10.93 & 0.0026 & ---- & \\
\hline WVU3-2 & Inj. & 42.5 & 57.5 & 3360 & 5.84 & --- & $4.02 \mathrm{E}+05$ & $4.019 \mathrm{E}+05$ \\
\hline
\end{tabular}

In the second case, $\mathrm{CO}_{2}$ is injected at 3360 psi for 59.5 years. As shown in Table 5-6, methane production is enhanced by $6.95 \%$ in WVU2-1 and $4.02 \mathrm{E}+05$ tons of $\mathrm{CO}_{2}$ are injected and completely stored in the formation with very low $\mathrm{CO}_{2}$ production (135.06 tons) form the producing well. The total production from WVU2-1 and WVU3-2 in this case goes to $16.77 \mathrm{Bcf}$, which is still below the total methane production in the base case.

Figure 5-9 shows the $\mathrm{CO}_{2}$ injection rate and compares the cumulative methane production before and after $\mathrm{CO}_{2}$ injection for Case2. The cum. $\mathrm{CH}_{4}$ production is started to increase after almost 20 years of injection, which is earlier than the scenario 2 due to lower distances between the production and injection wells. In addition, $\mathrm{CO}_{2}$ injection rate declines from $3020 \mathrm{Mscf} /$ day to around $200 \mathrm{Mscf} /$ day after 50 years of injection. 


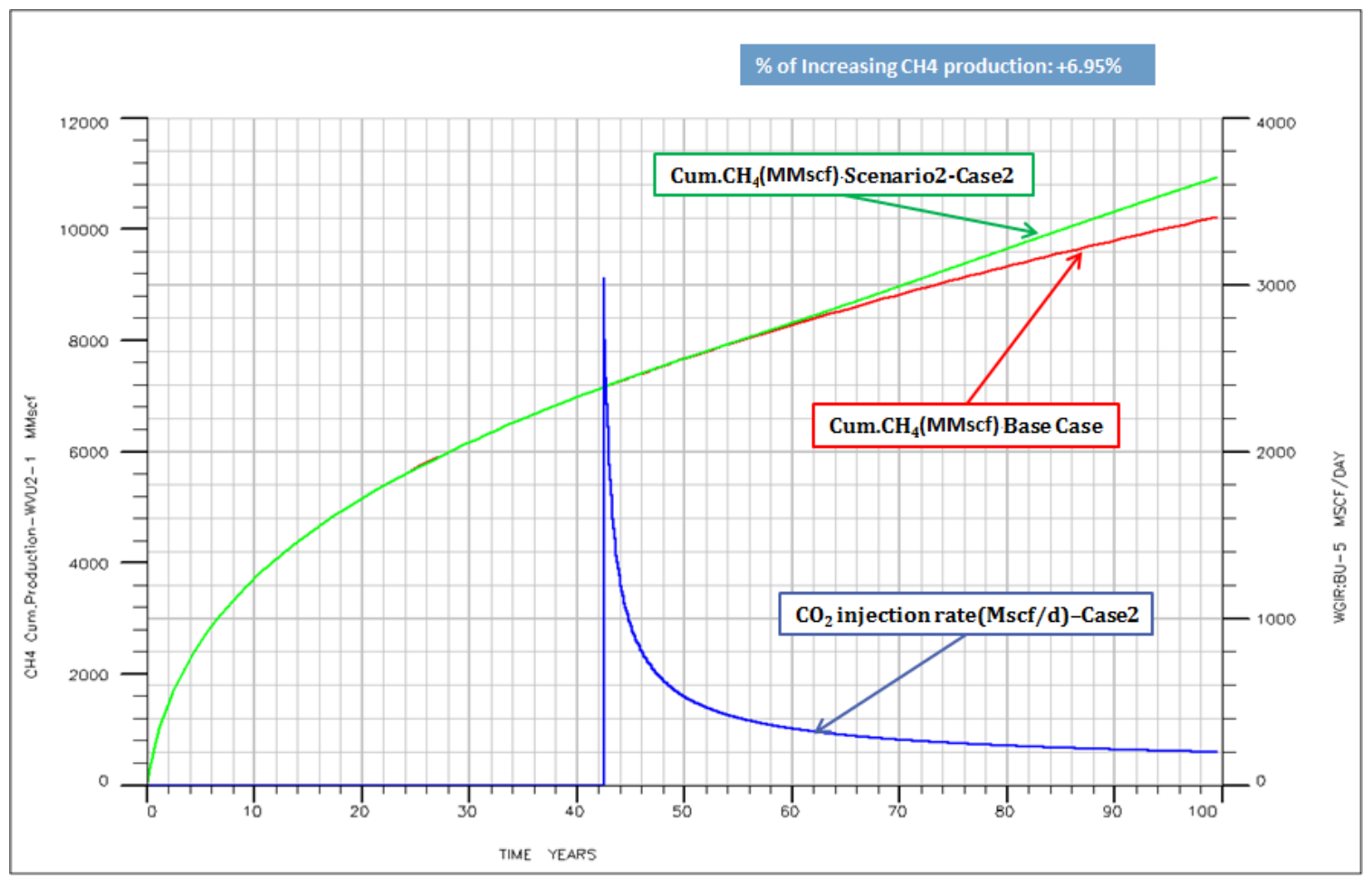

Figure 5-9. CO2 injection rate profile and Comparison of $\mathrm{CH} 4$ cumulative production in base case and Case 2

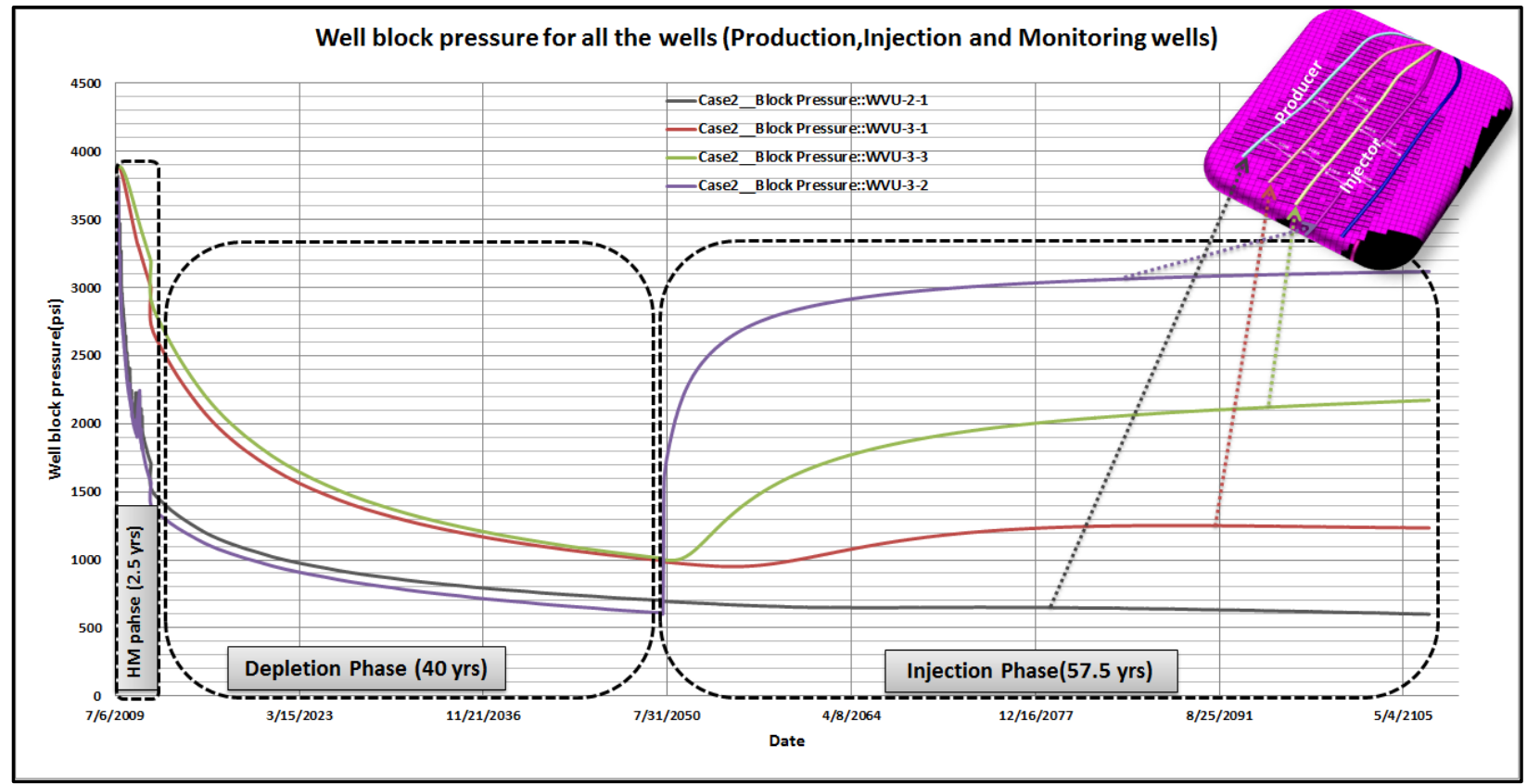

Figure 5-10.Well block pressure profile for all the wells during history matching, depletion and injection

Figure 5-10 shows the well block pressure changes during the history matching and depletion period and also during the injection for production and injection laterals as well as two monitoring wells in between. The maximum well block pressure belongs to the injector and is identified by purple line. 
The pressure support due to carbon dioxide injection, maintains the pressure in production well (black solid line).The block pressure profiles for two monitoring wells (WVU3-3 and WVU3-1) are shown with green and red solid lines correspondingly.

Figure 5-11 shows the $\mathrm{CH}_{4}$ and $\mathrm{CO}_{2}$ molar density changes in production lateral as well as two monitoring wells (WVU3-3 and WVU3-1). The same behavior, as was explained in the scenario 1, is observed here too. A sudden increase in molar density of methane in the closets monitoring well to the injector followed by a sharp decline due to re-adsorption of methane and then counter diffusion of $\mathrm{CO}_{2}$ $\mathrm{CH}_{4}$ is shown by blue solid line in Figure 5-11 (top).

Moreover, an increasing trend in the molar density of $\mathrm{CO}_{2}$ in the closet monitoring well to the injector can be seen from this graph (bottom). This increase in carbon dioxide molar density is very slow in the other monitoring wells due to the very slow diffusion of $\mathrm{CO}_{2}$ in the matrix. 


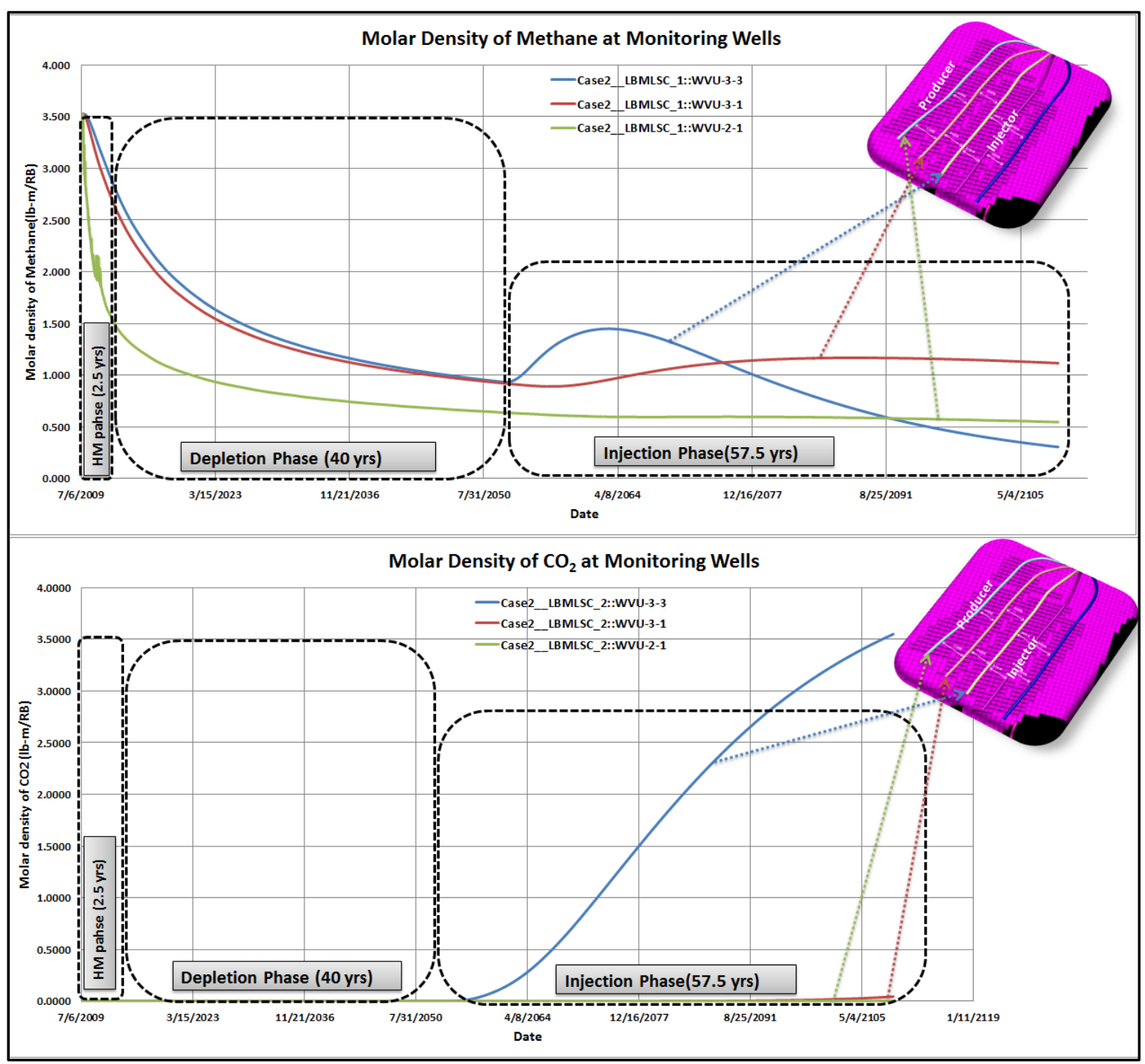

Figure 5-11.Methane and Carbon dioxide molar density changes in monitoring wells in scenario 2-Case2 (from top to bottom)

\subsection{3 $\mathrm{CO}_{2}$-EGR\&S- Scenario 3}

In the third scenario, still WVU2-1 stays as the production well, which is, located around $950 \mathrm{ft}$. away from WVU3-3 that is the injector and has 30 clusters with fracture conductivity of 0.95 to $4.58 \mathrm{md}-\mathrm{ft}$. Base case information for this scenario is summarized in Table 5-7.

According to this table, the prospective injection well (WVU3-3) is produced its $75 \%$ of accessible reserve after 46.5 years of production and the well block pressure is decline from initial reservoir pressure of 3890 psi to 416 psi. 
Table 5-7.Base injection case for scenario3

\begin{tabular}{cccccc}
\hline Well name & $\begin{array}{c}\text { Gas rate @ the } \\
\text { end of 100 } \\
\text { years(Mscf/d) }\end{array}$ & $\begin{array}{c}\text { 100 years } \\
\text { Cum. gas } \\
\text { production } \\
\text { (Bcf) }\end{array}$ & $\begin{array}{c}\text { 75\% of } \\
\text { Reachable } \\
\text { Reserve (Bcf) }\end{array}$ & $\begin{array}{c}\text { Time to reach } \\
\text { 75\% of } \\
\text { accessible } \\
\text { reserve(year) }\end{array}$ & $\begin{array}{c}\text { P_block after } \\
\text { 100 years(psi) }\end{array}$ \\
\hline WVU2-1 & 106 & 9.16 & 6.87 & 50.5 & 481 \\
WVU3-3 & 78 & 8.28 & 6.21 & 46.5 & 416 \\
\hline
\end{tabular}

In this scenario, two cases are defined like the previous scenarios based on different bottomhole injection pressure of 1680 and 3360 psi.

The results of $\mathrm{CO}_{2}$ injection in Case 1are summarized in Table 5-8 .According to this table, 1.00E+05 tons of $\mathrm{CO}_{2}$ are injected at a constant bottomhole pressure of 1680 psi for 53.5 years with low $\mathrm{CO}_{2}$ cum. production of 2451.9 tons but significant increase in comparison to the cases in scenario2. At $\mathrm{t}=78.2$ years, only $1 \%$ of daily gas production is carbon dioxide and this amount will be reached to $7.3 \%$ at $\mathrm{t}=100$ years.

Because of lower distance between the production and injection well, the more increase in production is achieved in this scenario compares to the previous ones and the methane production from WVU2-1 is enhanced by $6.0 \%$. However, the total methane production of 15.92 Bcf from WVU2-1 and WVU3-3 in Case1 is lower than the total production of $17.44 \mathrm{Bcf}$ in the senario3-base case.

Table 5-8. Injection scenario 3-Case 1: Duration of injection and the amount of recovered $\mathrm{CH}_{4}$ and stored $\mathrm{CO}_{2}$

\begin{tabular}{|c|c|c|c|c|c|c|c|c|}
\hline $\begin{array}{c}\text { Well } \\
\text { name }\end{array}$ & $\begin{array}{l}\text { Well } \\
\text { type }\end{array}$ & $\begin{array}{c}\text { Injection } \\
\text { starts } @ \\
\mathbf{t}=(\text { years })\end{array}$ & $\begin{array}{c}\text { Injection } \\
\text { period } \\
\text { (years) }\end{array}$ & $\begin{array}{l}\text { Injection } \\
\text { pressure } \\
\quad \text { (psi) }\end{array}$ & $\begin{array}{c}\mathrm{CH}_{4} \mathrm{Cum} \text {. } \\
\text { production } \\
\text { (Bcf) }\end{array}$ & $\begin{array}{c}\mathrm{CO}_{2} \mathrm{Cum} . \\
\text { production } \\
\text { (Bcf) }\end{array}$ & $\begin{array}{c}\text { Cum. } \\
\text { Injected } \\
\mathrm{CO}_{2} \\
\text { (ton) } \\
\end{array}$ & $\begin{array}{c}\text { Total } \\
\text { Stored } \\
\mathrm{CO}_{2} \\
\text { (ton) } \\
\end{array}$ \\
\hline WVU2-1 & Prod. & ------ & -------- & ------- & 9.71 & 0.0472 & ---- & \\
\hline WVU3-3 & Inj. & 46.5 & 53.5 & 1680 psi & 6.21 & --- & $1.00 \mathrm{E}+05$ & $9.76 \mathrm{E}+04$ \\
\hline
\end{tabular}

In the second case, $\mathrm{CO}_{2}$ is injected at 3360 psi for 53.5 years. As illustrated in Table 5-9, methane production is enhanced by $7.75 \%$ in WVU2-1 and $4.58 \mathrm{E}+05$ tons of $\mathrm{CO}_{2}$ are injected into the reservoir. $\mathrm{CO}_{2}$ breakthrough happens at $\mathrm{t}=68.19$ years when $10 \%$ of the total gas production are $\mathrm{CO}_{2}$. This amount will be increased significantly at $\mathrm{t}=100$ years and accounts for $40 \%$ of daily gas production.

The total methane production from WVU2-1 and WVU3-3 in this case reaches to16.08Bcf, which is still below the total methane production in the base case. 
Table 5-9. Injection scenario 3-Case 2: Duration of injection and the amount of recovered $\mathrm{CH} 4$ and stored $\mathrm{CO} 2$

\begin{tabular}{|c|c|c|c|c|c|c|c|c|}
\hline $\begin{array}{c}\text { Well } \\
\text { name }\end{array}$ & $\begin{array}{l}\text { Well } \\
\text { type }\end{array}$ & $\begin{array}{c}\text { Injection } \\
\text { starts } @ \\
\text { t= (years) }\end{array}$ & $\begin{array}{c}\text { Injection } \\
\text { period } \\
\text { (years) }\end{array}$ & $\begin{array}{c}\text { Injection } \\
\text { pressure } \\
\text { (psi) }\end{array}$ & $\begin{array}{c}\mathrm{CH}_{4} \mathrm{Cum} . \\
\text { production } \\
\text { (Bcf) } \\
\end{array}$ & $\begin{array}{c}\mathrm{CO}_{2} \mathrm{Cum} . \\
\text { production } \\
\text { (Bcf) } \\
\end{array}$ & $\begin{array}{c}\text { Cum. } \\
\text { Injected } \\
\mathrm{CO}_{2} \text { (ton) }\end{array}$ & $\begin{array}{c}\text { Total } \\
\text { Stored } \\
\mathrm{CO}_{2} \text { (ton) } \\
\end{array}$ \\
\hline WVU2-1 & Prod. & ------ & -------- & ------- & 9.87 & 0.908 & ---- & ----- \\
\hline WVU3-3 & Inj. & 46.5 & 53.5 & 3360 & 6.21 & --- & $4.58 \mathrm{E}+05$ & $4.11 \mathrm{E}+05$ \\
\hline
\end{tabular}

$\mathrm{CO}_{2}$ injection rate and comparison of the cumulative methane production before and after $\mathrm{CO}_{2}$ injection for Case2 are illustrated in Figure 5-12. As shown in this plot, the cum. methane production is started to increase after almost nine years of injection, which is much faster than the previous scenarios because of very low distances between the production and injection well. Furthermore, $\mathrm{CO}_{2}$ injection rate declines from $3450 \mathrm{Mscf} /$ day to around $250 \mathrm{Mscf} /$ day after 50 years of injection.

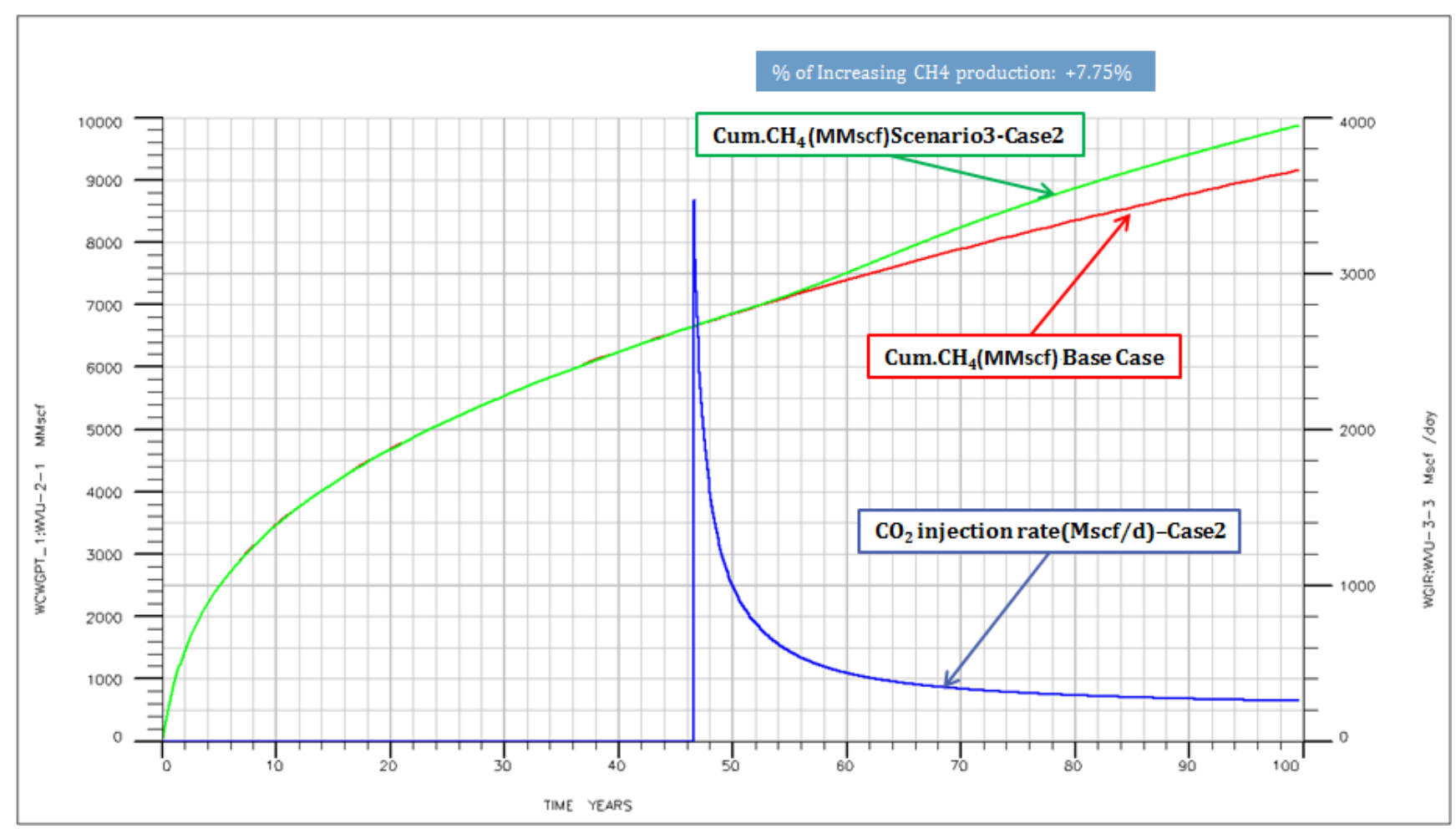

Figure 5-12.CO2 injection rate profile and Comparison of $\mathrm{CH} 4$ cumulative production in base case and Case 3

Methane and carbon dioxide molar density changes in production well (WVU2-1) and in the monitoring well (WVU3-1) are shown in Figure 5-13 (from top to bottom relatively). The same behavior, as the previous scenarios is observed here as well. 


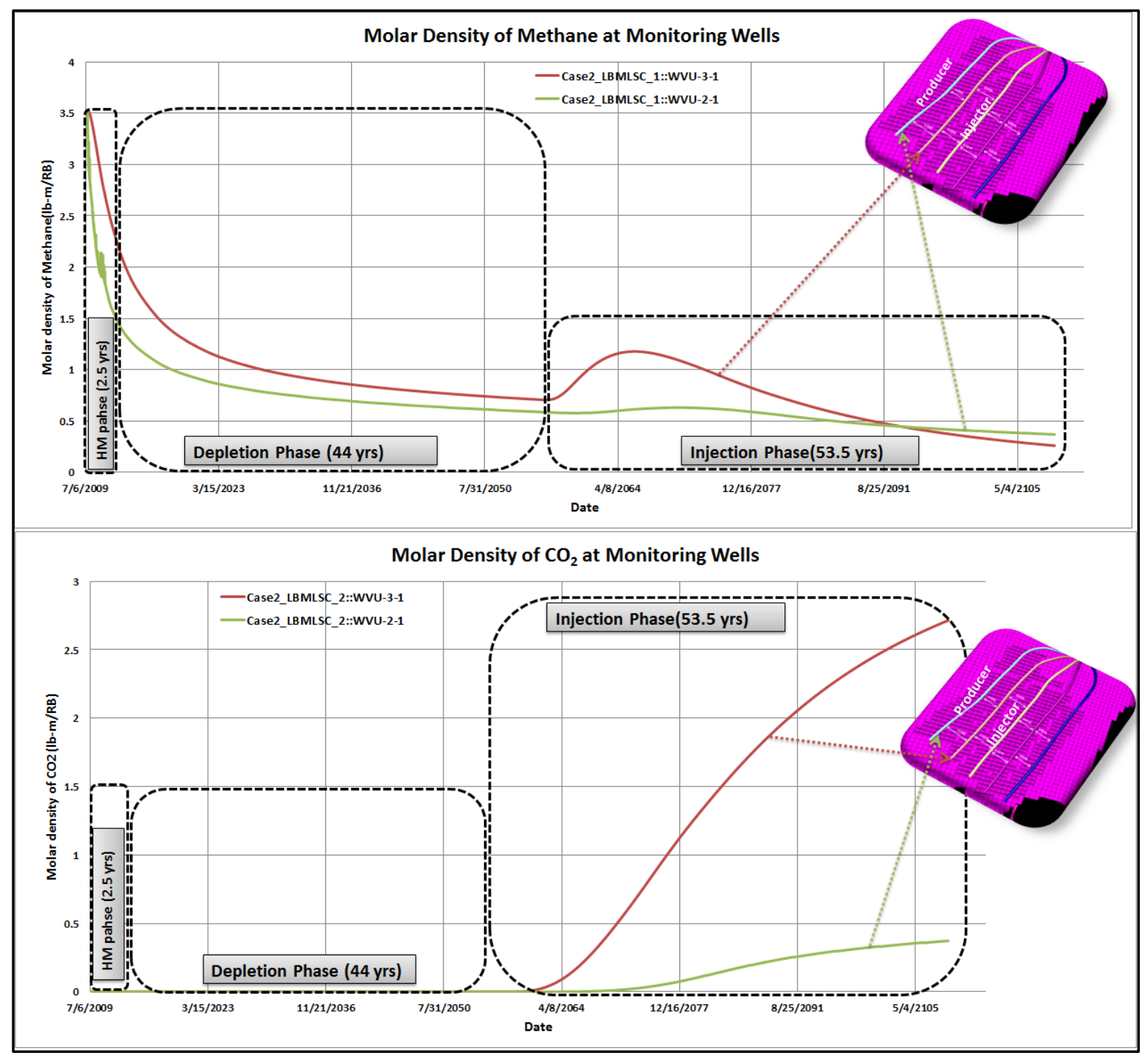

Figure 5-13.Methane and Carbon dioxide molar density changes in monitoring wells in scenario 3-Case2 (from top to bottom)

\subsection{4 $\mathrm{CO}_{2}$-EGR\&S- Scenario 4}

The objective of designing the fourth scenario is to show the inefficiency of $\mathrm{CO}_{2}$-EGR\&S process in the case of selecting the injector and producer so close to each other that the overlapped fracture can communicate easily. In this scenario, WVU2-1 is the production well, which is located very close to WVU3-1 as an injector and has 30 clusters of hydraulic fracture. Figure 5-14 shows the schematics and relative distances between producer and injector in this scenario. According to the information in Table 5-10, the prospective injection well (WVU3-1) produced its $75 \%$ of accessible reserve after 54.5 years of production and the well block pressure is declined from initial reservoir pressure of 3890 psi to 423.5 psi. 


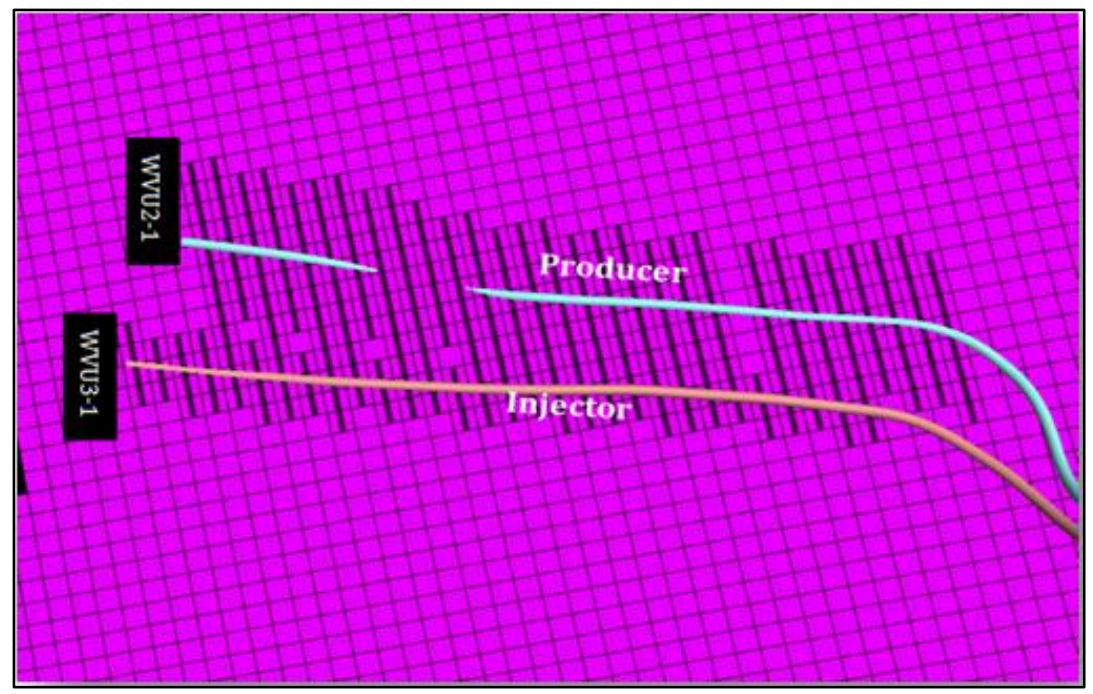

Figure 5-14-Production and injection wells schematic in Scenario4

The injection is started at this point by injecting $\mathrm{CO}_{2}$ at a constant bottomhole injection pressure of 1680 , which is $25 \%$ of lithostatic pressure. The results are summarized in the Table 5-11.According to this table; $1.86 \mathrm{E}+5$ tons of $\mathrm{CO}_{2}$ are injected during 44.5 yrs., while the only $5.5 \mathrm{E}+4$ tons of it are stored in the formation. Therefore, very early breakthrough, which is because of strong communications between the overlapped hydraulic fractures, makes this type of design impractical from an economic point of view (Due to very high separation cost of $\mathrm{CO}_{2}$ from the gas produced gas stream).

Table 5-10.Base injection case for scenario3

\begin{tabular}{cccccc}
\hline Well name & $\begin{array}{c}\text { Gas rate @ the } \\
\text { end of 100 } \\
\text { years(Mscf/d) }\end{array}$ & $\begin{array}{c}\text { 100 years } \\
\text { Cum. gas } \\
\text { production } \\
\text { (Bcf) }\end{array}$ & $\begin{array}{c}\text { 75\% of } \\
\text { Reachable } \\
\text { Reserve (Bcf) }\end{array}$ & $\begin{array}{c}\text { Time to reach } \\
\text { 75\% of } \\
\text { accessible } \\
\text { reserve(year) }\end{array}$ & $\begin{array}{c}\text { P_block after } \\
\text { 100 years(psi) }\end{array}$ \\
\hline WVU2-1 & 101.4 & 8.28 & 6.21 & 52.5 & 470.7 \\
WVU3-1 & 85.2 & 6.75 & 5.06 & 54.5 & 423.5 \\
\hline
\end{tabular}

Table 5-11. Injection scenario 4: Duration of injection and the amount of recovered $\mathrm{CH}_{4}$ and stored $\mathrm{CO}_{2}$

\begin{tabular}{|c|c|c|c|c|c|c|c|c|}
\hline $\begin{array}{c}\text { Well } \\
\text { name }\end{array}$ & $\begin{array}{l}\text { Well } \\
\text { type }\end{array}$ & $\begin{array}{c}\text { Injection } \\
\text { starts } @ \\
\mathbf{t}=(\text { years })\end{array}$ & $\begin{array}{c}\text { Injection } \\
\text { period } \\
\text { (years) }\end{array}$ & $\begin{array}{c}\text { Injection } \\
\text { pressure } \\
\text { (psi) }\end{array}$ & $\begin{array}{c}\mathrm{CH}_{4} \mathrm{Cum} \text {. } \\
\text { production } \\
\text { (Bcf) }\end{array}$ & $\begin{array}{c}\mathrm{CO}_{2} \mathrm{Cum} . \\
\text { production } \\
\text { (Bcf) }\end{array}$ & $\begin{array}{c}\text { Cum. } \\
\text { Injected } \\
\mathrm{CO}_{2} \\
\text { (ton) } \\
\end{array}$ & $\begin{array}{c}\text { Total } \\
\text { Stored } \\
\mathrm{CO}_{2} \\
\text { (ton) } \\
\end{array}$ \\
\hline WVU2-1 & Prod. & ------ & -------- & ------- & 8.77 & 2.52 & ---- & ----- \\
\hline WVU3-3 & Inj. & 55.5 & 44.5 & $1680 \mathrm{psi}$ & 5.066 & ------- & $1.86 \mathrm{E}+5$ & $5.5 \mathrm{E}+4$ \\
\hline
\end{tabular}




\subsection{5 $\mathrm{CO}_{2}$-EGR\&S- Scenario 5}

In the fifth and the last scenario that is designed and investigated in this chapter, two production wells and one injector in between are used. WVU2-1 and WVU2-2 are selected as the production laterals, which are located about 970 and $930 \mathrm{ft}$. respectively away from the WVU3-3 as an injector.WVU2-1, WVU2-2 and WVU3-3 are hydraulically fractured and have 29, 27 and 30 clusters respectively.

Base case information in this scenario is summarized in Table 5-12. In this table, the prospective injection well (WVU3-3) is produced its 75\% of accessible reserves after 33.49 years of production and its block pressure is declined from initial reservoir pressure of 3890 psi to 300.4 psi. The total methane production from these three wells after 100 years of production reaches to $19.5 \mathrm{Bcf}$. The best injection case should maximize methane production from the other production wells as a result of $\mathrm{CO}_{2}$ injection to the extent that compensate the methane production from the well that is converted to the injection well during its productive life, in addition to maximizing the amount of carbon dioxide in the formation.

Table 5-12.Base injection case for scenario5

\begin{tabular}{cccccc}
\hline Well name & $\begin{array}{c}\text { Gas rate @ the } \\
\text { end of 100 } \\
\text { years(Mscf/d) }\end{array}$ & $\begin{array}{c}\text { 100 years } \\
\text { Cum. gas } \\
\text { production } \\
\text { (Bcf) }\end{array}$ & $\begin{array}{c}\text { 75\% of } \\
\text { Reachable } \\
\text { Reserve (Bcf) }\end{array}$ & $\begin{array}{c}\text { Time to reach } \\
\mathbf{7 5 \%} \text { of } \\
\text { accessible } \\
\text { reserve(year) }\end{array}$ & $\begin{array}{c}\text { P_block after } \\
\text { 100 years(psi) }\end{array}$ \\
\hline WVU2-1 & 102.38 & 8.98 & 6.73 & 49.5 & 474.3 \\
WVU3-3 & 38.42 & 5.57 & 4.18 & 33.49 & 300.4 \\
WVU2-2 & 29.64 & 4.99 & 3.74 & 32.5 & 380.6 \\
\hline
\end{tabular}

In the first case of this scenario, $\mathrm{CO}_{2}$ is injected at a constant bottomhole injection pressure of $1680 \mathrm{psi}$ ( $25 \%$ of lithostatic pressure).

Table 5-13summarizes the results of $\mathrm{CO}_{2}$ injection in the Case 1. According to this table, $2.08 \mathrm{E}+05$ tons of $\mathrm{CO}_{2}$ are injected for 66.51 years with very low $\mathrm{CO}_{2}$ breakthrough of $4.26 \mathrm{E}+03 \mathrm{Bcf}$. Therefore, more than $97 \%$ of the injected $\mathrm{CO}_{2}$ is stored in the reservoir and methane production from WVU2-1 and WVU2-2 are enhanced by 7.57 and $10.8 \%$, respectively. At $t=100$ years, 10.4 percent of daily gas production is $\mathrm{CO}_{2}$ that is an acceptable percentage from the operational and economic pint of view. Moreover, the total methane production from all the wells is reached to $19.37 \mathrm{Bcf}$, which is very close to the total production of $19.54 \mathrm{Bcf}$ in the senario5-base case. By comparing this result with the all previous cases in four different scenarios, it can be concluded that this injection design is the best one so far, for the purpose of $\mathrm{CO}_{2}$-EGR and storage. 
Table 5-13. Injection scenario 5-Case 1: Duration of injection and the amount of recovered $\mathrm{CH}_{4}$ and stored $\mathrm{CO}_{2}$

\begin{tabular}{|c|c|c|c|c|c|c|c|c|}
\hline $\begin{array}{c}\text { Well } \\
\text { name }\end{array}$ & $\begin{array}{l}\text { Well } \\
\text { type }\end{array}$ & $\begin{array}{c}\text { Injection } \\
\text { starts @ } \\
\mathbf{t}= \\
\text { (years) } \\
\end{array}$ & $\begin{array}{c}\text { Injection } \\
\text { period } \\
\text { (years) }\end{array}$ & $\begin{array}{l}\text { Injection } \\
\text { pressure } \\
\quad \text { (psi) }\end{array}$ & $\begin{array}{l}\mathrm{CH}_{4} \mathrm{Cum} . \\
\text { production } \\
\text { (Bcf) }\end{array}$ & $\begin{array}{c}\mathrm{CO}_{2} \mathrm{Cum} . \\
\text { production } \\
\text { (Bcf) }\end{array}$ & $\begin{array}{c}\text { Cum. } \\
\text { Injected } \\
\mathrm{CO}_{2} \\
\text { (ton) }\end{array}$ & $\begin{array}{c}\text { Total } \\
\text { Stored } \\
\mathrm{CO}_{2} \\
\text { (ton) } \\
\end{array}$ \\
\hline WVU2-1 & Producer & ----- & ----- & ----- & 9.66 & 0.082 & ----- & \\
\hline WVU3-3 & Injector & 33.49 & 66.51 & 1680 & 4.18 & ------- & $2.08 \mathrm{E}+05$ & $2.02 \mathrm{e}+5$ \\
\hline WVU2-2 & Producer & ----- & ----- & ----- & 5.53 & 0.027 & ----- & \\
\hline
\end{tabular}

In order to identify the maximum allowable bottomhole injection pressure for optimizing $\mathrm{CO}_{2}$-EGR\&S process, Case 2 is designed to inject $\mathrm{CO}_{2}$ at a constant bottomhole pressure of 2520 that accounts for $37.5 \%$ of lithostatic pressure. Table 5-14 summarizes the result $\mathrm{CO}_{2}$ injection in this case.

Table 5-14 Injection scenario 5-Case 2: Duration of injection and the amount of recovered $\mathrm{CH}_{4}$ and stored $\mathrm{CO}_{2}$

\begin{tabular}{|c|c|c|c|c|c|c|c|c|}
\hline $\begin{array}{l}\text { Well } \\
\text { name }\end{array}$ & $\begin{array}{l}\text { Well } \\
\text { type }\end{array}$ & $\begin{array}{c}\text { Injection } \\
\text { starts } @ \\
\text { t }= \\
\text { (years) }\end{array}$ & $\begin{array}{c}\text { Injection } \\
\text { period } \\
\text { (years) }\end{array}$ & $\begin{array}{c}\text { Injection } \\
\text { pressure } \\
\text { (psi) }\end{array}$ & $\begin{array}{c}\mathrm{CH}_{4} \mathrm{Cum} . \\
\text { production } \\
\text { (Bcf) }\end{array}$ & $\begin{array}{c}\mathrm{CO}_{2} \mathrm{Cum} . \\
\text { production } \\
\text { (Bcf) }\end{array}$ & $\begin{array}{c}\text { Cum. } \\
\text { Injected } \\
\mathrm{CO}_{2}(\text { ton })\end{array}$ & $\begin{array}{c}\text { Total } \\
\text { Stored } \\
\mathrm{CO}_{2} \\
\text { (ton) }\end{array}$ \\
\hline WVU2-1 & Producer & ---- & ---- & ---- & 9.806 & 0.644 & ---- & \\
\hline WVU3-3 & Injector & 33.49 & 66.51 & 2520 & 4.18 & ------- & $4.34 \mathrm{E}+05$ & $3.8 e+5$ \\
\hline WVU2-2 & Producer & ----- & ----- & ----- & 5.69 & 0.392 & ----- & \\
\hline
\end{tabular}

As shown in Table 5-14, methane production from WVU2-1 and WVU2-2 is enhanced by 9.2 and 14.02 $\%$ correspondingly, and $4.34 \mathrm{E}+05$ tons of $\mathrm{CO}_{2}$ are injected into the reservoir and about $87.6 \%$ of total injected $\mathrm{CO}_{2}$ is stored in the formation.

A Carbon dioxide breakthrough happens at $\mathrm{t}=65$.9years and $\mathrm{t}=69.2$ years in WVU2-1 and WVU2-2 respectively, when $10 \%$ of the total gas production are $\mathrm{CO}_{2}$. This amount will be increased significantly and accounts for 36.2 and $58.4 \%$ of daily gas production at $\mathrm{t}=100$ years in WVU2-1 and WVU2-2 correspondingly. The total $\mathrm{CO}_{2}$ production in both producers after at $\mathrm{t}=100$ years reach to $5.38 \mathrm{E}+04$ tons.

Additionally, the total methane production from all the wells is increased to $19.67 \mathrm{Bcf}$, which is even slightly beyond the total methane production of $19.54 \mathrm{Bcf}$ in the senario5-base case. 
Comparisons of the cumulative methane production from WVU2-1 and WVU2-2 before and after $\mathrm{CO}_{2}$ injection and $\mathrm{CO}_{2}$ injection rate for Case 1 are illustrated in Figure 5-15 and Figure 5-16 respectively. As shown in these plots, the cum. methane productions are started to increase after 17 and 14 years of injection, in WVU2-1 and WVU2-2.

Additionally, $\mathrm{CO}_{2}$ injection rate declines from $1005 \mathrm{Mscf} /$ day to around 120Mscf/day after 30 years of injection in Case 1, while in the second case, the injection rate is changing from $2400 \mathrm{Mscf} / \mathrm{d}$ at the beginning to the around $230 \mathrm{Mscf} / \mathrm{d}$ after 37 years of injection.

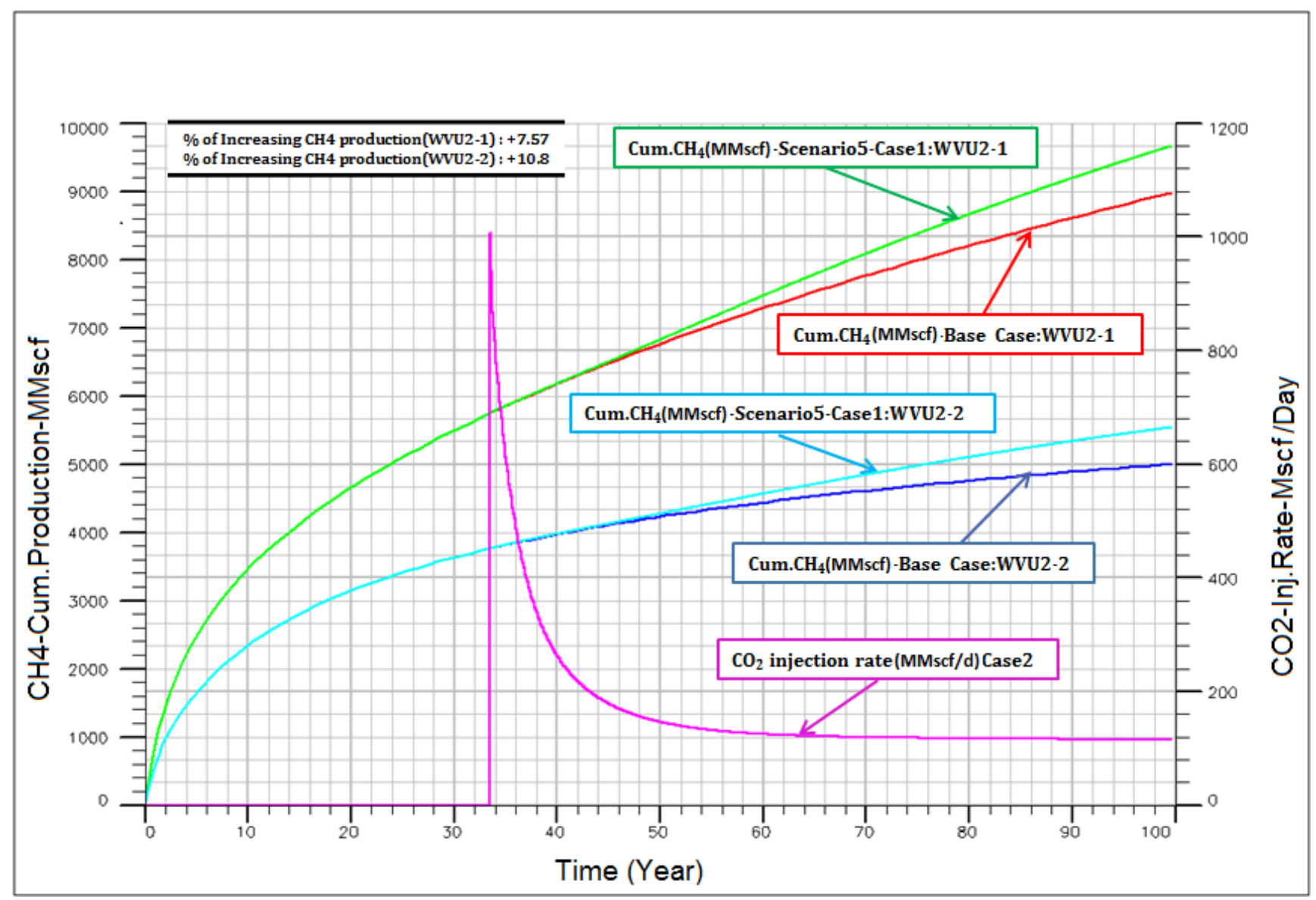

Figure 5-15.CO2 injection rate profile and Comparison of $\mathrm{CH}_{4}$ cumulative production in base case and Case 1 


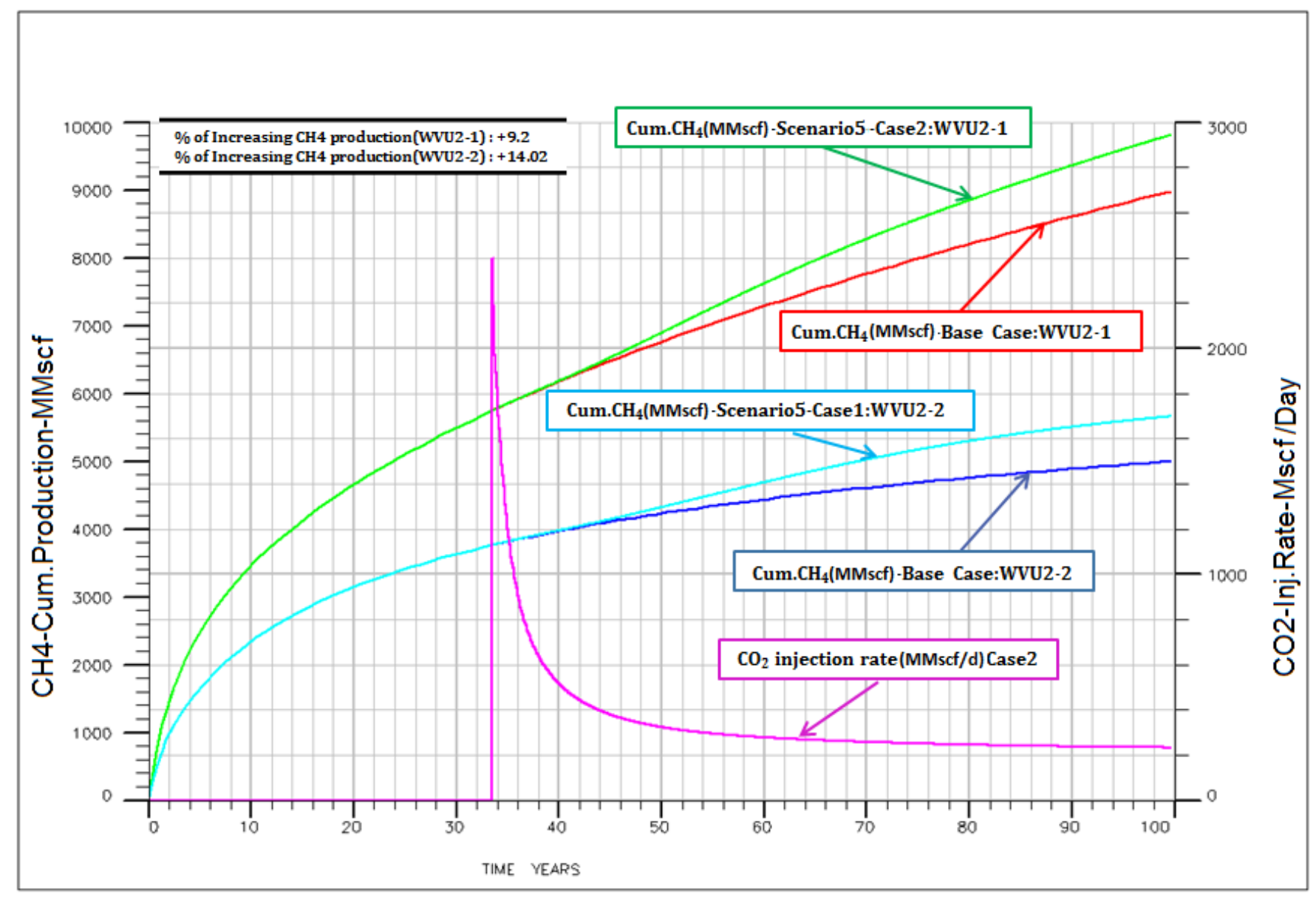

Figure 5-16. $\mathrm{CO} 2$ injection rate profile and Comparison of $\mathrm{CH} 4$ cumulative production in base case and Case 2

Molar density distribution of $\mathrm{CO}_{2}$ and $\mathrm{CH}_{4}$ after 1,3,5,10,20 and 50 years of $\mathrm{CO}_{2}$ injection for the scenario 5 are shown in Figure 5-17 and Figure 5-18, correspondingly. The scale for the molar densities for both $\mathrm{CO}_{2}$ and $\mathrm{CH}_{4}$ are from 0 to 0.07 (mole per reservoir volume).

In both figures, the purple and red colors show the minimum and maximum values for molar density of $\mathrm{CO}_{2}$ and $\mathrm{CH}_{4}$.

As can be seen in this graph after 50 years of $\mathrm{CO}_{2}$ injection, the $\mathrm{CO}_{2}$ plume extension does not go beyond the stimulated reservoir volume around the injection well. Therefore, pressurized, super critical $\mathrm{CO}_{2}$ is stored in the organic and inorganic pores and the extension of $\mathrm{CO}_{2}$ plume is depending on the extension of hydraulic fractures in the injection well. 


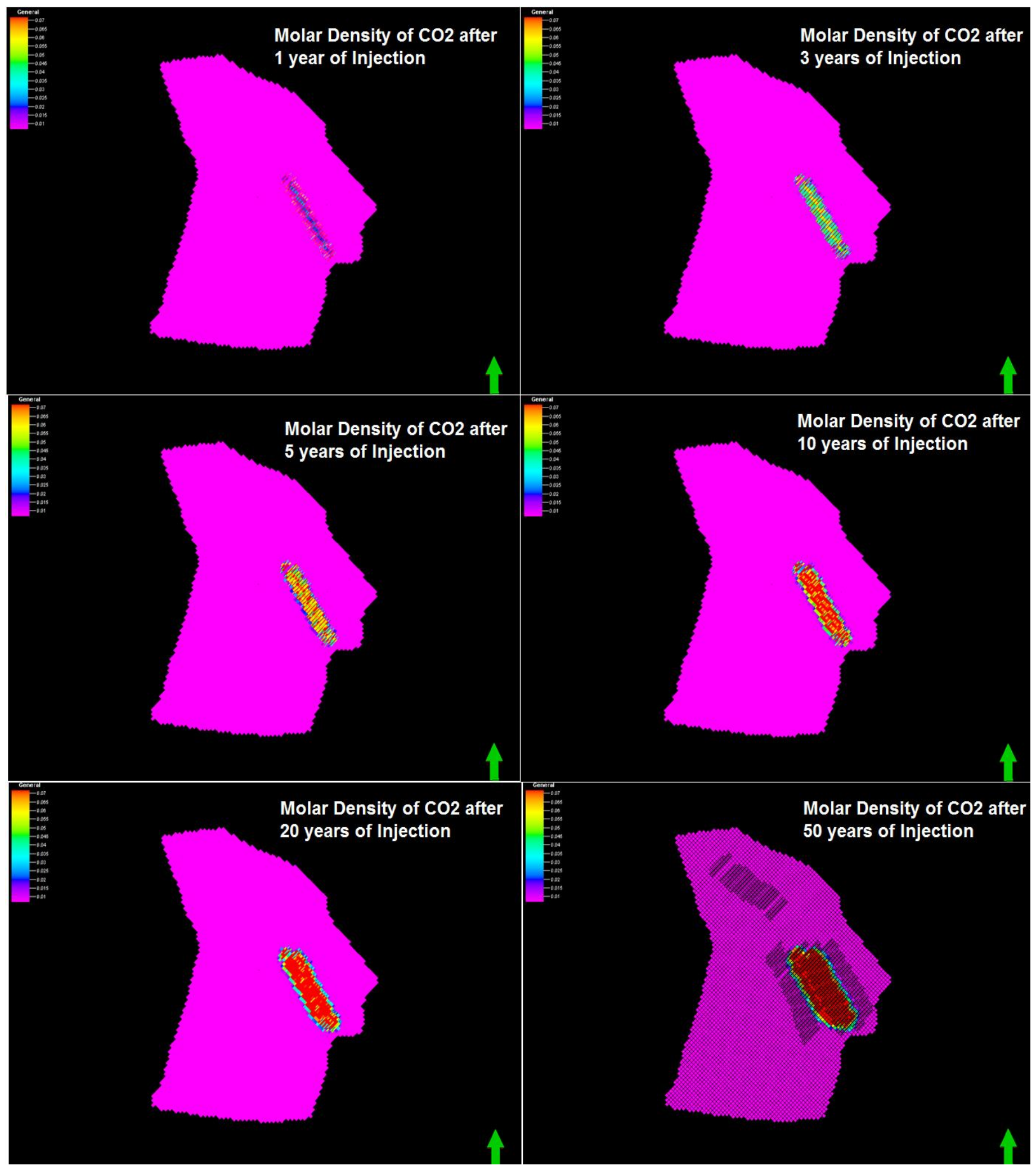

Figure 5-17. Molar density of $\mathrm{CO} 2$ after $1,3,5,10,20$ and 50 years of injection 


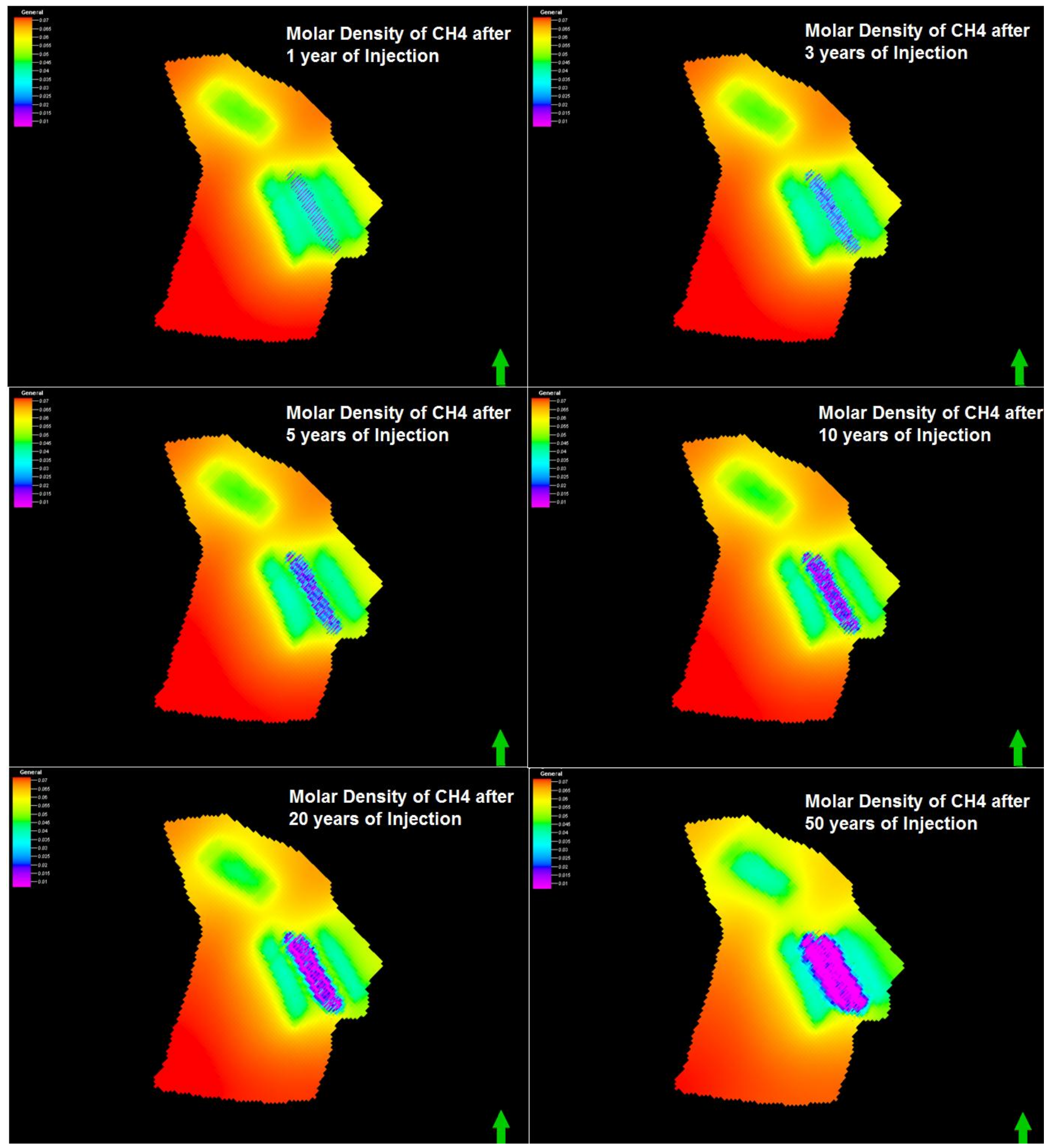

Figure 5-18. Molar density of Methane after 1,3,5,10,20 and 50 years of injection 


\subsection{Effect of Sorption Isotherm on $\mathrm{CO}_{2}$-EGR\&S Process}

One of the key components of a $\mathrm{CO}_{2}$-EGR\&S process is considering the impact of sorption isotherms. The isotherm represents the thermodynamic limit of how much carbon dioxide or methane can be stored in shale at a given pressure.

In order to express the relative adsorption capacities of $\mathrm{CH}_{4}$ and $\mathrm{CO}_{2}$, the separation factor or $\mathrm{CO}_{2}-\mathrm{CH}_{4}$ competitive adsorption Capacity is defined and expressed as:

$$
\alpha_{(\mathrm{CO} 2-\mathrm{CH} 4 \text { Competetive Ads.Capacity })}=\frac{\left(\mathrm{V}_{\mathrm{L}-\mathrm{CO} 2} * \mathrm{P}_{\mathrm{L}-\mathrm{CH} 4}\right)}{\left(\mathrm{V}_{\mathrm{L}-\mathrm{CH} 4} * \mathrm{P}_{\mathrm{L}-\mathrm{CO} 2}\right)}
$$

One of the scenarios from the previous section (Scenario 3) is used to perform the sensitivity analysis on $\mathrm{CO}_{2}$ and $\mathrm{CH}_{4}$ Langmuir isotherms in order to identify their impact on cumulative methane production, total injected $\mathrm{CO}_{2}$ and total $\mathrm{CO}_{2}$ breakthrough. In the base case scenario, $0.073 \mathrm{Mscf} /$ ton and 726 psi are used as the $\mathrm{CH}_{4}$ Langmuir volume and pressure, respectively and $0.12 \mathrm{Mscf} / \mathrm{ton}$ and $400 \mathrm{psi}$ are defined as the $\mathrm{CO}_{2}$ Langmuir volume and pressure, correspondingly. In order to perform the sensitivity analysis on each isotherm component $\left(\mathrm{P}_{\mathrm{L}}, \mathrm{V}_{\mathrm{L}}\right)$ for $\mathrm{CO}_{2}$ and $\mathrm{CH}_{4}$, two additional simulation runs are defined and $\mathrm{P}_{\mathrm{L}}$ and $\mathrm{V}_{\mathrm{L}}$ for $\mathrm{CO}_{2}$ and $\mathrm{CH}_{4}$ are changed by $50 \%$ above and below the base case.

\subsection{1 $\quad \mathrm{CH}_{4}$ Sorption Volume}

Table 5-15 summarizes the isotherm values that are used to perform the sensitivity analysis on methane sorption volume. In this table, the selectivity ratio or $\mathrm{CO}_{2}-\mathrm{CH}_{4}$ competitive adsorption Capacity $(\alpha)$ is calculated for each case. Figure 5-19 shows the cumulative methane production results obtained in three cases. As shown in this figure, increasing the methane Langmuir volume yields an increase in the initial gas in place (sorbed gas) that improves total methane production (MMscf).

Table 5-15- Longmuir isotherms and selectivity ratio for sensitivity cases on VL-CH4

\begin{tabular}{|c|c|c|c|c|c|}
\hline & \multicolumn{2}{|c|}{ Methane } & \multicolumn{2}{|c|}{ Carbon dioxide } & \multirow[t]{2}{*}{ Selectivity ratio( $\alpha)$} \\
\hline & $V_{L}(M s c f / t o n)$ & $P_{L}(p s i)$ & $V_{L}(M s c f / t o n)$ & $P_{L}(p s i)$ & \\
\hline Base Case & 0.073 & 726 & 0.12 & 400 & 2.98 \\
\hline SA-V ${ }_{L_{-}} \mathrm{CH}_{4}-$ Case1 & 0.110 & 726 & 0.12 & 400 & 1.99 \\
\hline $\mathrm{SA}-\mathrm{V}_{\mathrm{L}} \mathrm{CH}_{4}-\mathrm{Case}_{2}$ & 0.037 & 726 & 0.12 & 400 & 5.97 \\
\hline
\end{tabular}




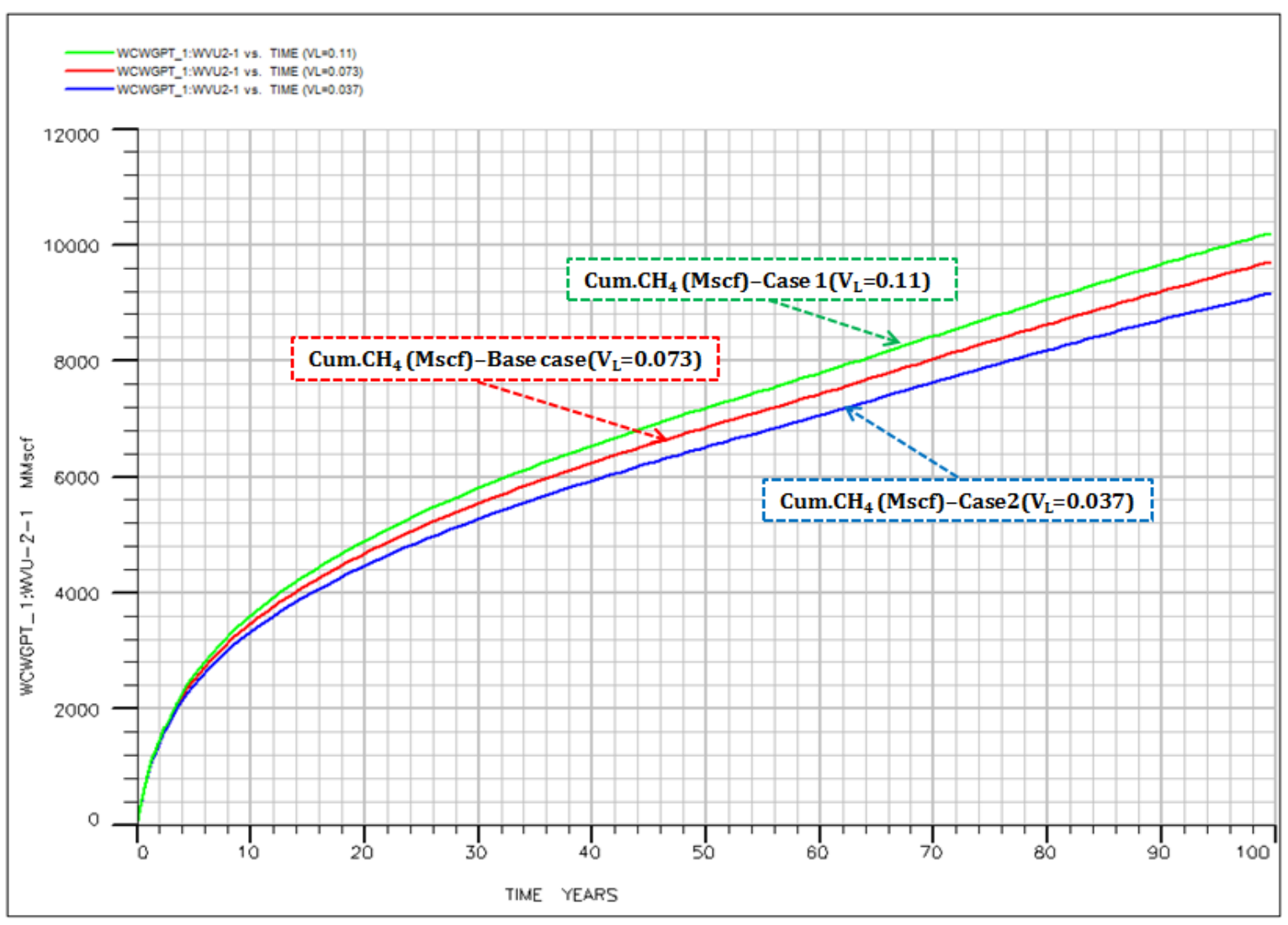

Figure 5-19.Effect of different methane Langmuir volumes on $\mathrm{CH} 4$ cum production

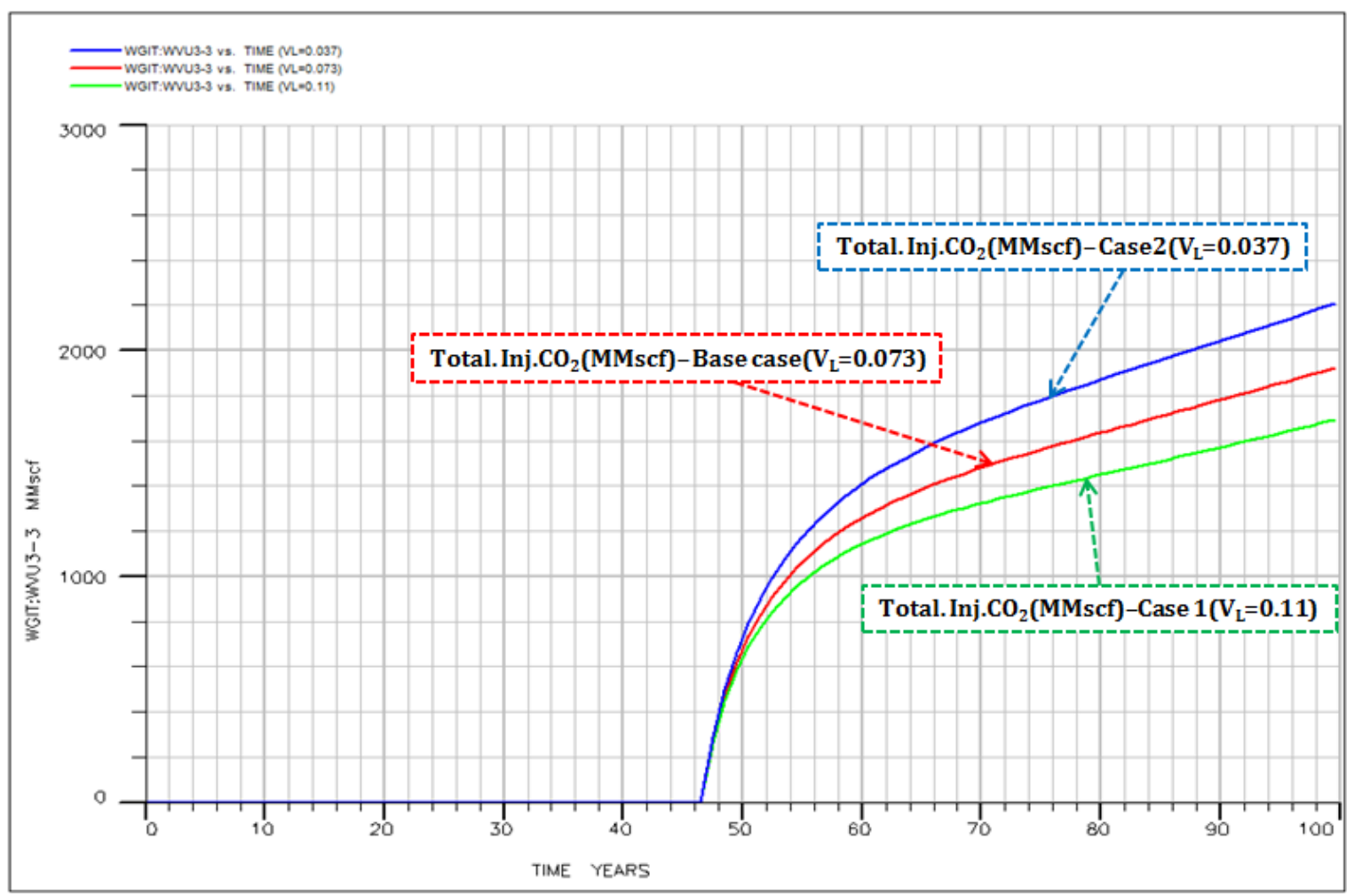

Figure 5-20.Effect of different methane Langmuir volumes on CO2 injectivity 
Figure 5-20 illustrates the impact of increasing methane Langmuir volume on total $\mathrm{CO}_{2}$ injectivity in the reservoir. It can be seen from this figure that raising the sorption volume of $\mathrm{CH}_{4}$ yields a decrease in $\mathrm{CO}_{2}$ $\mathrm{CH}_{4}$ competitive adsorption Capacity $(\alpha)$, which reduces the total amount of injected $\mathrm{CO}_{2}$ in the shale reservoir.

The impact of changing methane Langmuir volume on $\mathrm{CO}_{2}$ breakthrough is shown in Figure 5-21. In this figure two different zones are identified, which shows different $\mathrm{CO}_{2}$ production behaviors based on different $\mathrm{CH}_{4}$ Langmuir volume. By increasing the methane Langmuir volume, the selectivity ratio is decreased, therefore faster $\mathrm{CO}_{2}$ breakthrough is expected (Region1).

However, almost 20 years after the initial $\mathrm{CO}_{2}$ breakthrough, the reverse behavior is observed (Region 2). It is due to the fact that by lowering the $\mathrm{CH}_{4}$ Langmuir volume, the selectivity ratio is increased, therefore more $\mathrm{CO}_{2}$ is generally injected into the reservoir and an increase in $\mathrm{CO}_{2}$ production is observed during the last 10 years of injection.

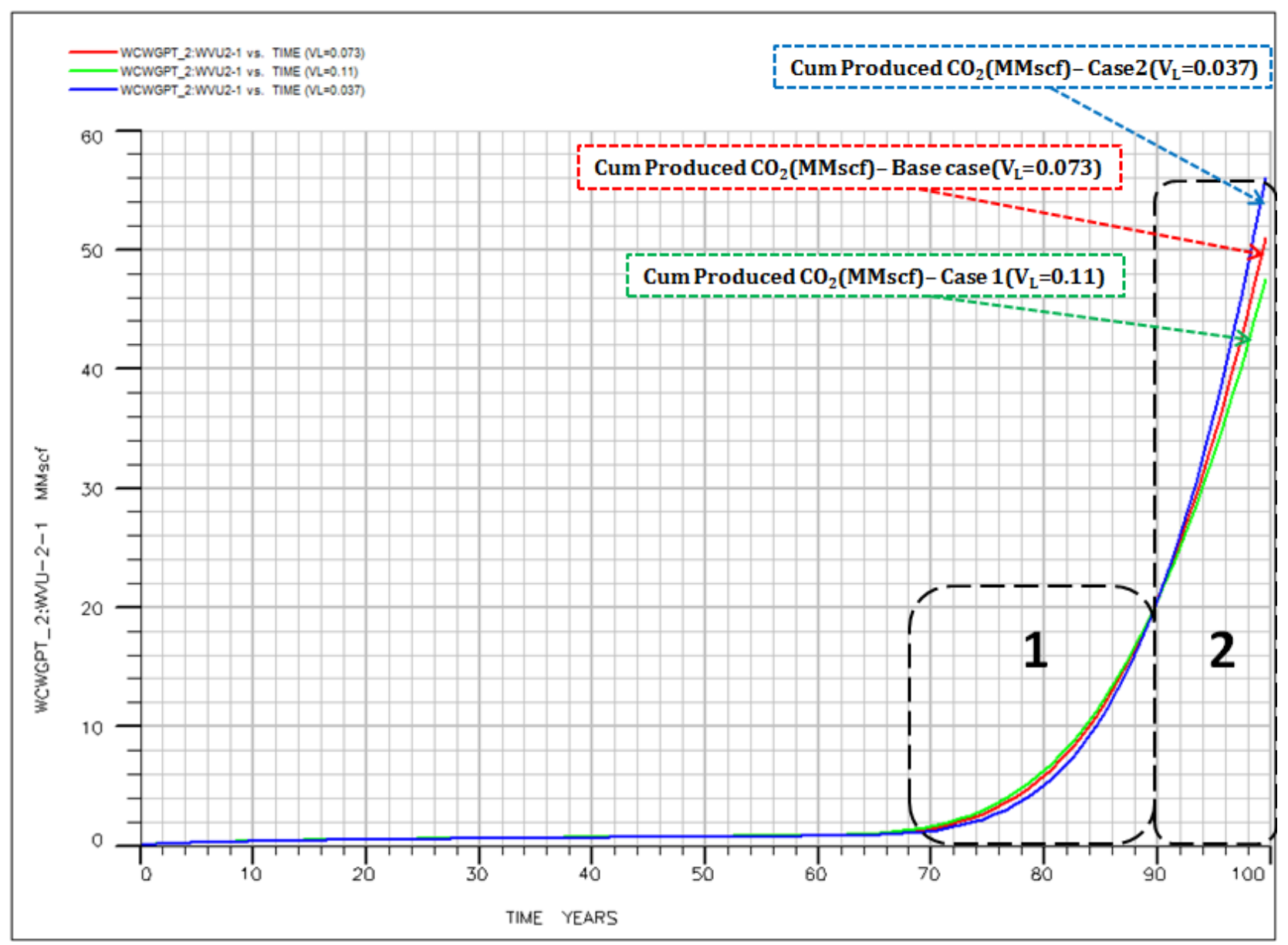

Figure 5-21.Effect of different methane Langmuir volumes on $\mathrm{CO} 2$ breakthrough 


\subsection{2 $\mathrm{CH}_{4}$ Sorption Pressure}

The isotherm values that are used to perform the sensitivity analysis on methane Langmuir pressure and the calculated selectivity ratio for all three cases are summarized in Table 5-16.

The cumulative methane production results obtained for three cases are shown in Figure 5-22.According to this figure, increasing the methane Langmuir pressure yields a slight increase in total methane production (MMscf).

Table 5-16- Longmuir isotherms and selectivity ratio for sensitivity cases on PL-CH4

\begin{tabular}{|c|c|c|c|c|c|}
\hline & \multicolumn{2}{|c|}{ Methane } & \multicolumn{2}{|c|}{ Carbon dioxide } & \multirow[t]{2}{*}{ Selectivity ratio( $\alpha)$} \\
\hline & $V_{L}(M s c f / t o n)$ & $P_{L}(p s i)$ & $V_{L}($ Mscf/ton $)$ & $P_{L}(p s i)$ & \\
\hline Base Case & 0.073 & 726 & 0.12 & 400 & 2.98 \\
\hline SA-P ${ }_{L_{-}} C_{4}-$ Case 1 & 0.073 & 1089 & 0.12 & 400 & 4.48 \\
\hline $\mathrm{SA}-\mathrm{P}_{\mathrm{L}_{-}} \mathrm{CH}_{4}-\mathrm{Case} 2$ & 0.073 & 363 & 0.12 & 400 & 1.49 \\
\hline
\end{tabular}

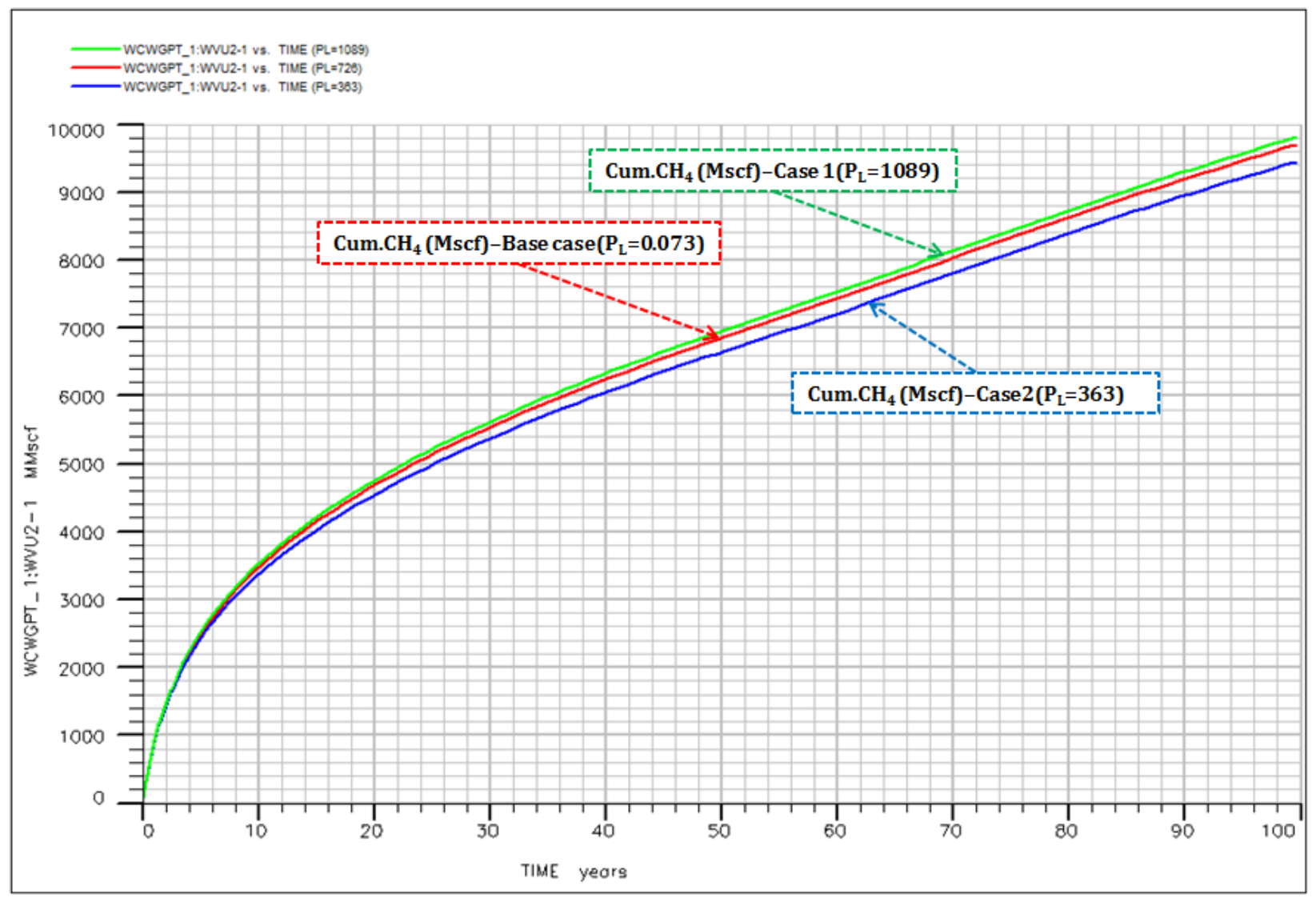

Figure 5-22.Effect of different methane Langmuir pressures on $\mathrm{CH} 4$ cum production 
Variations in Langmuir pressure of methane and their impact on total $\mathrm{CO}_{2}$ injectivity in the reservoir is shown in Figure 5-23. According to this figure, by increasing the $\mathrm{CH}_{4}$ Langmuir pressure, the selectivity ratio is increased and as a result increases in total injected $\mathrm{CO}_{2}$ in the reservoir is observed.

Figure 5-24 shows the sensitivity analysis results for changing methane Langmuir pressures and their impact on total $\mathrm{CO}_{2}$ production. As shown in this figure, the higher Langmuir pressure of methane causes an increase in selectivity ratio, which results in slower breakthrough time and less $\mathrm{CO}_{2}$ production.

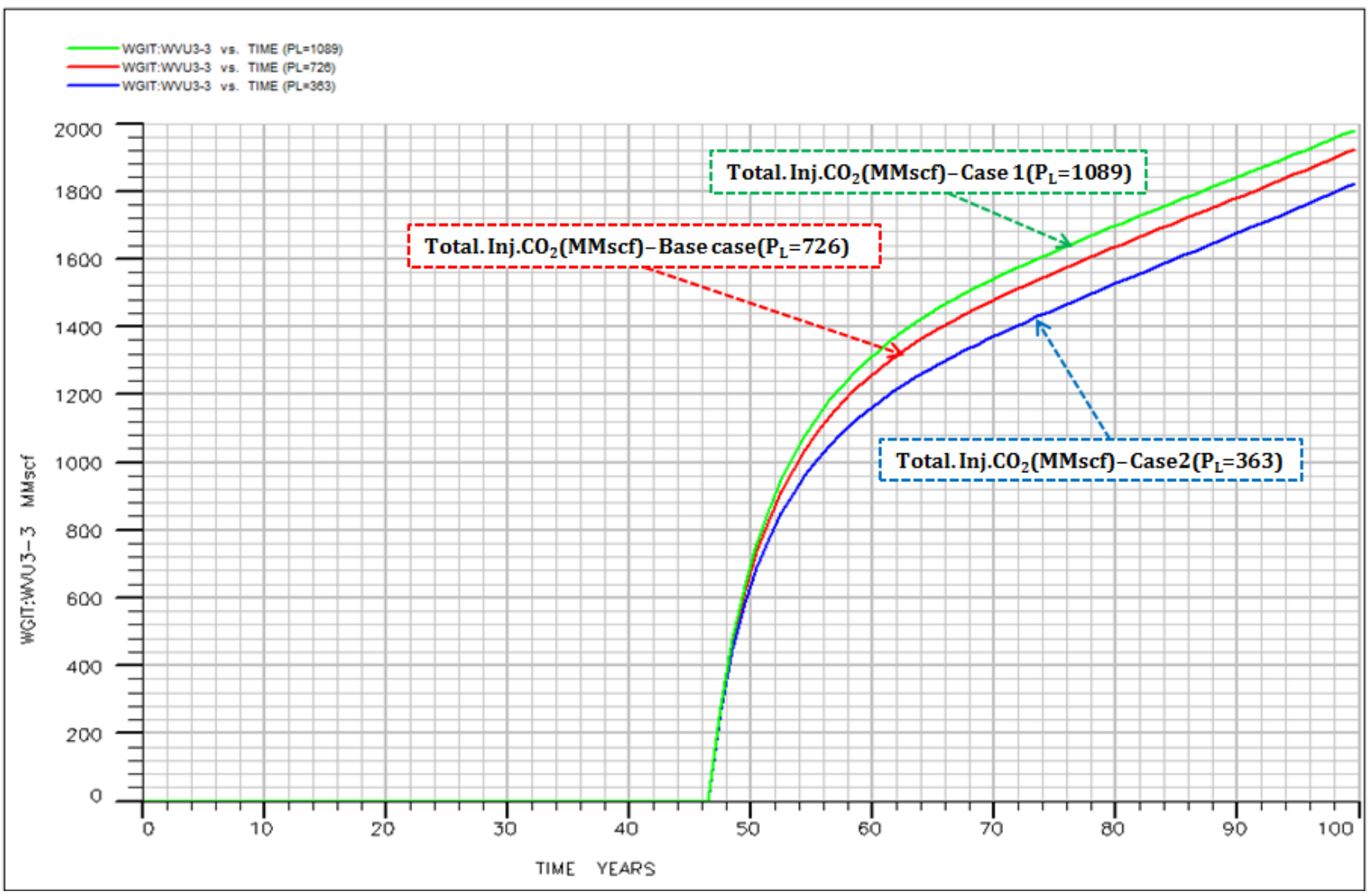

Figure 5-23.Effect of different methane Langmuir pressures on $\mathrm{CO} 2$ injectivity

\subsection{3 $\mathrm{CO}_{2}$ Sorption Volume}

Table 5-17 summarizes the isotherm values and resulting selectivity ratios that are used to perform sensitivity analysis on carbon dioxide sorption volume. The analysis shows that any changes on Langmuir pressure of $\mathrm{CO}_{2}$ does not have a significant impact on total methane recovery. However, any increase in $\mathrm{CO}_{2}$ Langmuir volume, causes an increase in selectivity ratio and, therefore higher volume of injected $\mathrm{CO}_{2}$ in the reservoir can be attained (Figure 5-25). 


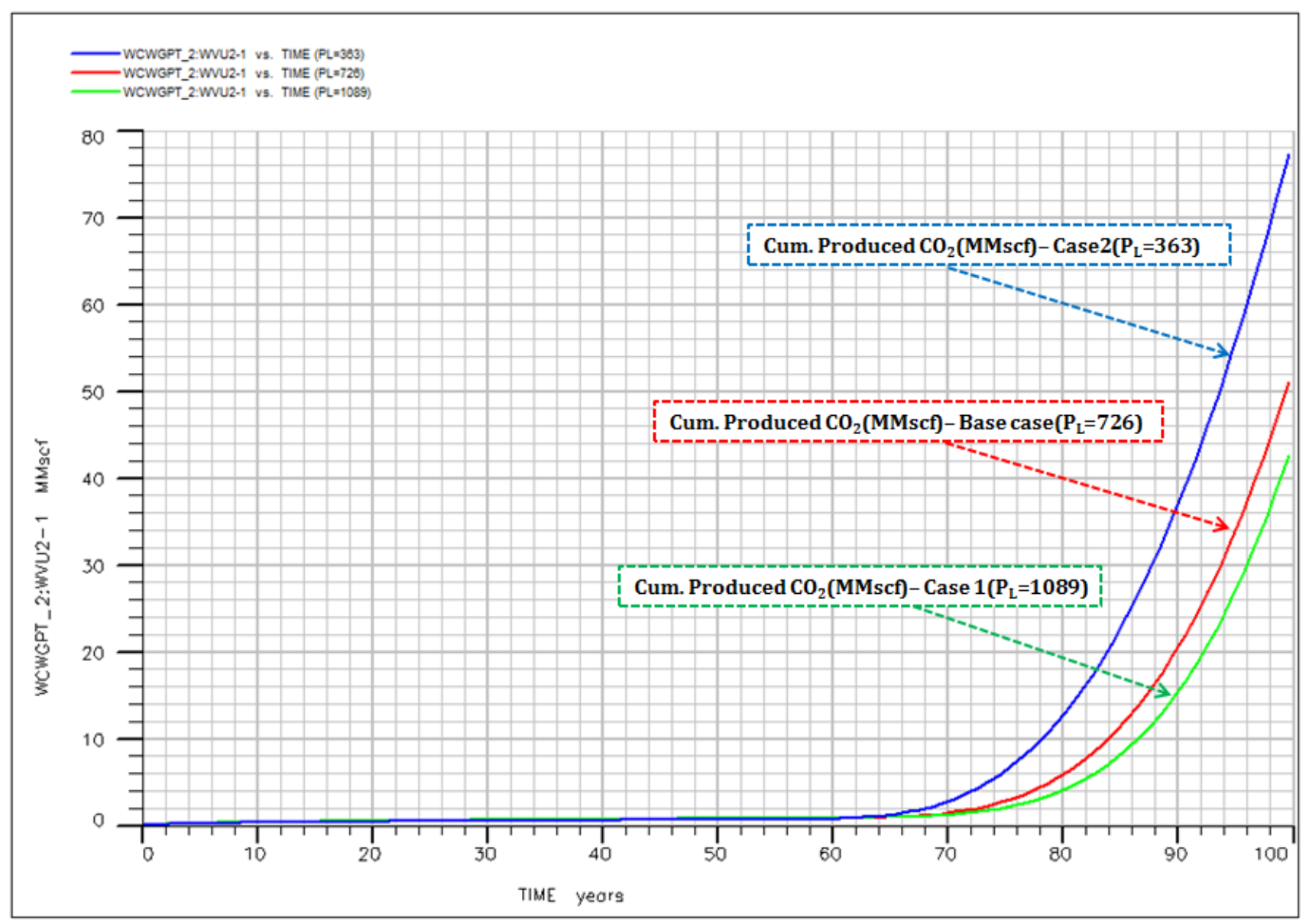

Figure 5-24.Effect of different methane Langmuir pressures on $\mathrm{CO} 2$ breakthrough

Figure 5-26 illustrates the results of varying $\mathrm{CO}_{2}$ Langmuir pressure and its impact on total $\mathrm{CO}_{2}$ production. It can be seen from this figure that lower $\mathrm{V}_{\mathrm{L}}$ results in a lower selectivity ratio and faster and higher $\mathrm{CO}_{2}$ breakthrough in production well.

Table 5-17- Longmuir isotherms and selectivity ratio for sensitivity cases on VL-CO2

\begin{tabular}{|c|c|c|c|c|c|}
\hline & \multicolumn{2}{|c|}{ Methane } & \multicolumn{2}{|c|}{ Carbon dioxide } & \multirow[t]{2}{*}{ Selectivity ratio( $\alpha)$} \\
\hline & $V_{L}(M s c f / t o n)$ & $P_{L}(p s i)$ & $V_{L}(M s c f / t o n)$ & $P_{L}(p s i)$ & \\
\hline Base Case & 0.073 & 726 & 0.12 & 400 & 2.98 \\
\hline SA-V ${ }_{L_{-}} \mathrm{CO}_{2^{-}}$-Case1 & 0.073 & 726 & 0.18 & 400 & 4.48 \\
\hline SA-V ${ }_{\mathrm{L}} \mathrm{CO}_{2}-\mathrm{Case} 2$ & 0.073 & 726 & 0.06 & 400 & 1.49 \\
\hline
\end{tabular}




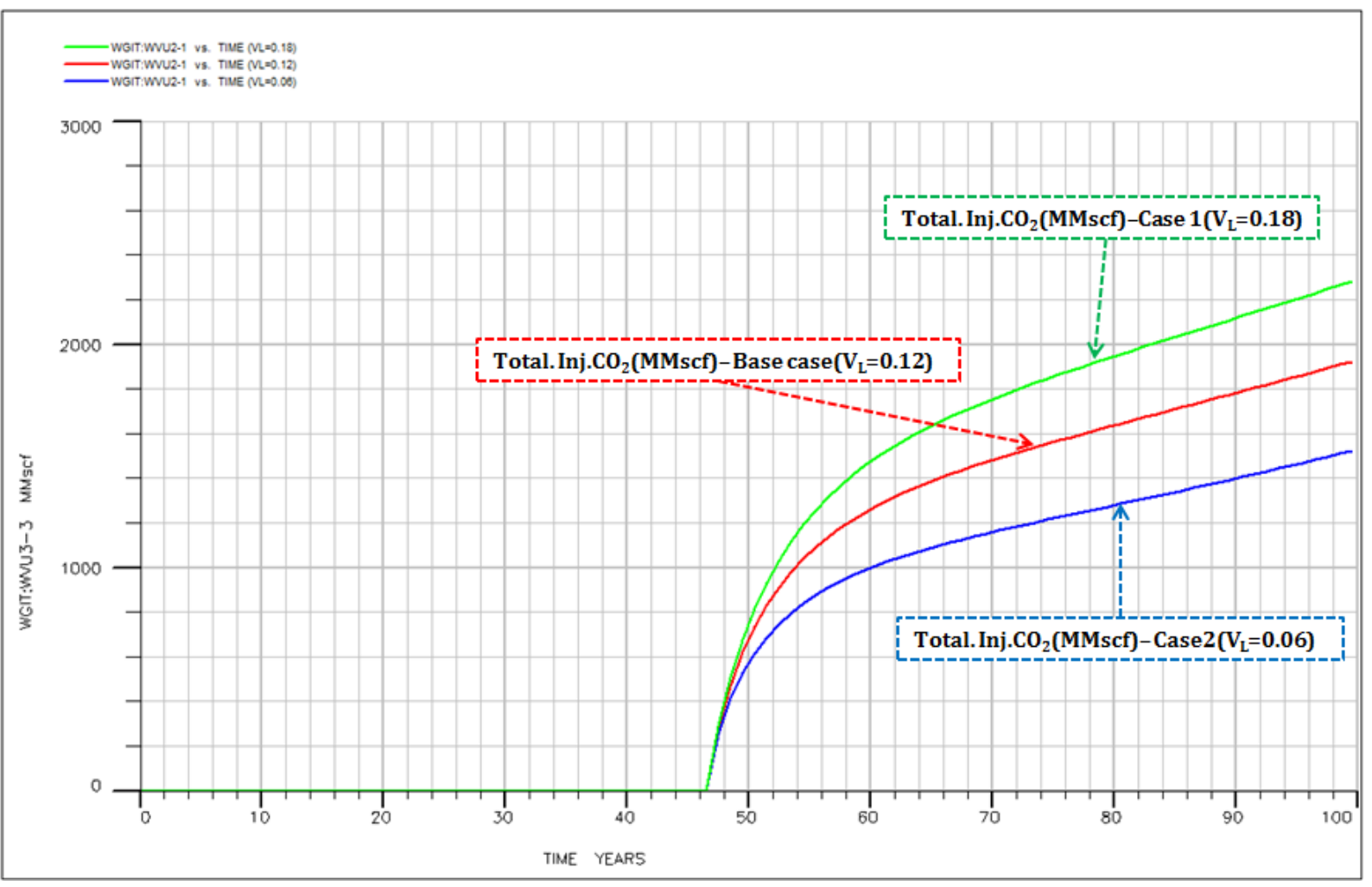

Figure 5-25. Effect of different Carbone dioxide Langmuir volume on $\mathrm{CO} 2$ injectivity

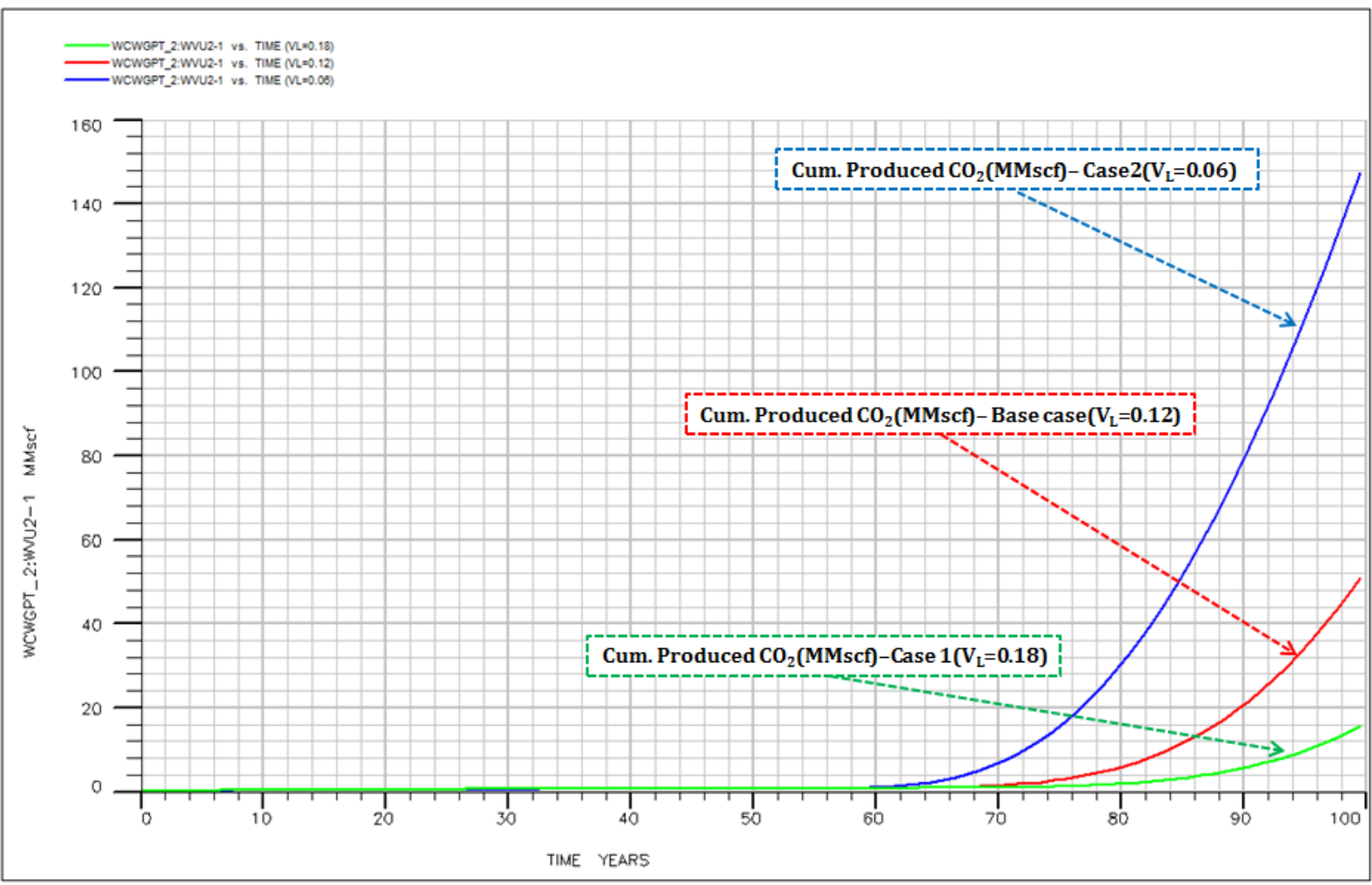

Figure 5-26.Effect of different carbon dioxide Langmuir volume on $\mathrm{CO} 2$ breakthrough 


\subsection{4 $\quad \mathrm{CO}_{2}$ Sorption Pressure}

Table 5-17 summarizes the isotherm values and resulting selectivity ratios that are used to perform sensitivity analysis on carbon dioxide sorption pressure. The analysis shows that the changing Langmuir pressure of $\mathrm{CO}_{2}$ does not have any impact on total methane recovery. Nevertheless, as can be seen in Figure 5-27, by lowering the $\mathrm{CO}_{2}$ Langmuir pressure, the selectivity ratio is increased, which causes an increase in volume of injected $\mathrm{CO}_{2}$ in the reservoir and also delays the $\mathrm{CO}_{2}$ breakthrough in the production lateral (Figure 5-28).

Table 5-18- Longmuir isotherms and selectivity ratio for sensitivity cases on PL-CO2

\begin{tabular}{|c|c|c|c|c|c|}
\hline & \multicolumn{2}{|c|}{ Methane } & \multicolumn{2}{|c|}{ Carbon dioxide } & \multirow[t]{2}{*}{ Selectivity ratio( $\alpha)$} \\
\hline & $V_{L}(M s c f / t o n)$ & $P_{L}(p s i)$ & $V_{L}(M s c f / t o n)$ & $P_{L}(p s i)$ & \\
\hline Base Case & 0.073 & 726 & 0.12 & 400 & 2.98 \\
\hline $\mathrm{SA}-\mathrm{P}_{\mathbf{L}_{-}} \mathrm{CO}_{2}$-Case1 & 0.073 & 726 & 0.12 & 600 & 1.99 \\
\hline SA-P ${ }_{L_{-}} \mathrm{CO}_{2}$-Case 2 & 0.073 & 726 & 0.12 & 200 & 5.97 \\
\hline
\end{tabular}

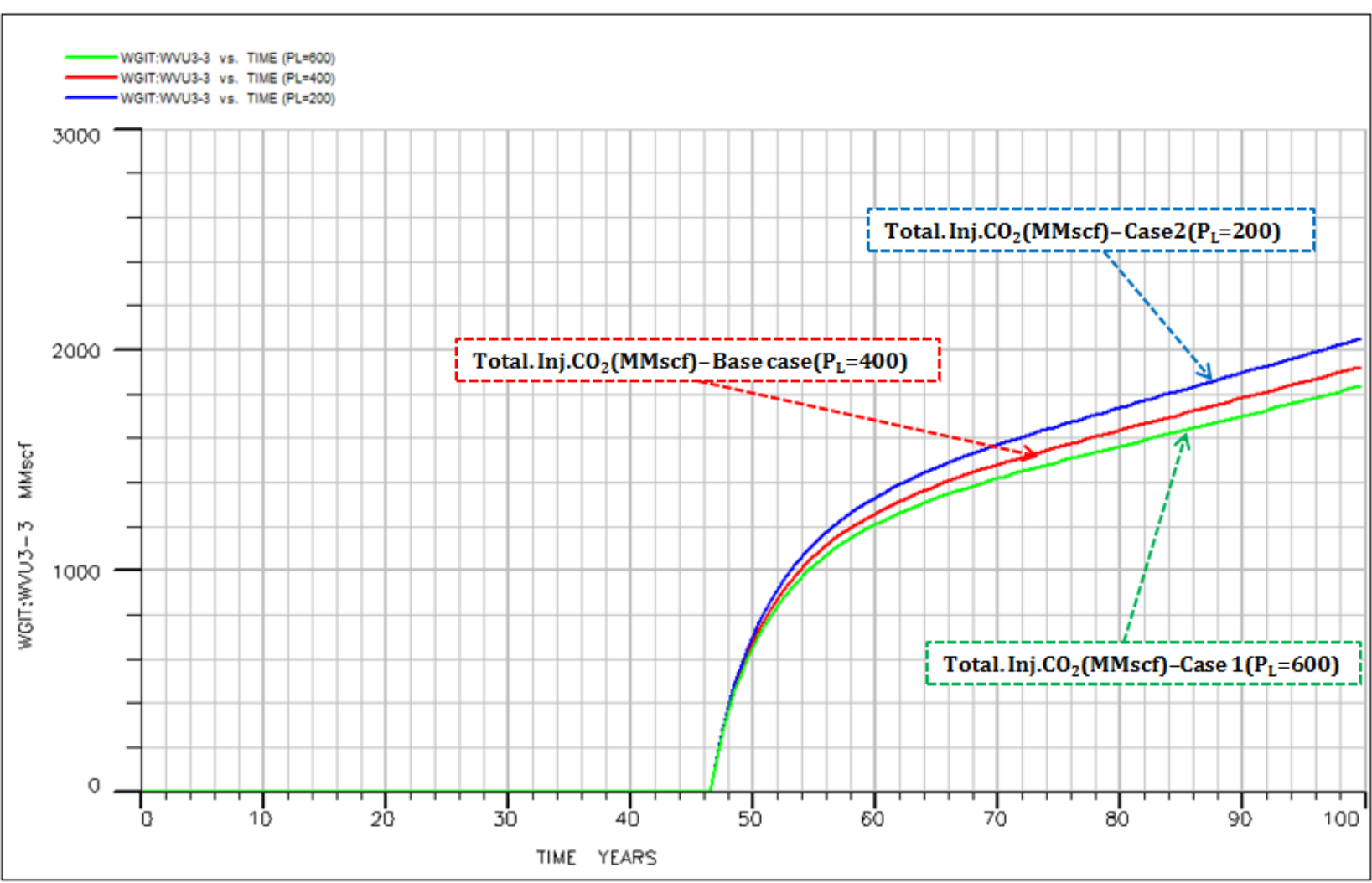

Figure 5-27.Effect of different Carbone dioxide Langmuir pressure on $\mathrm{CO} 2$ injectivity 


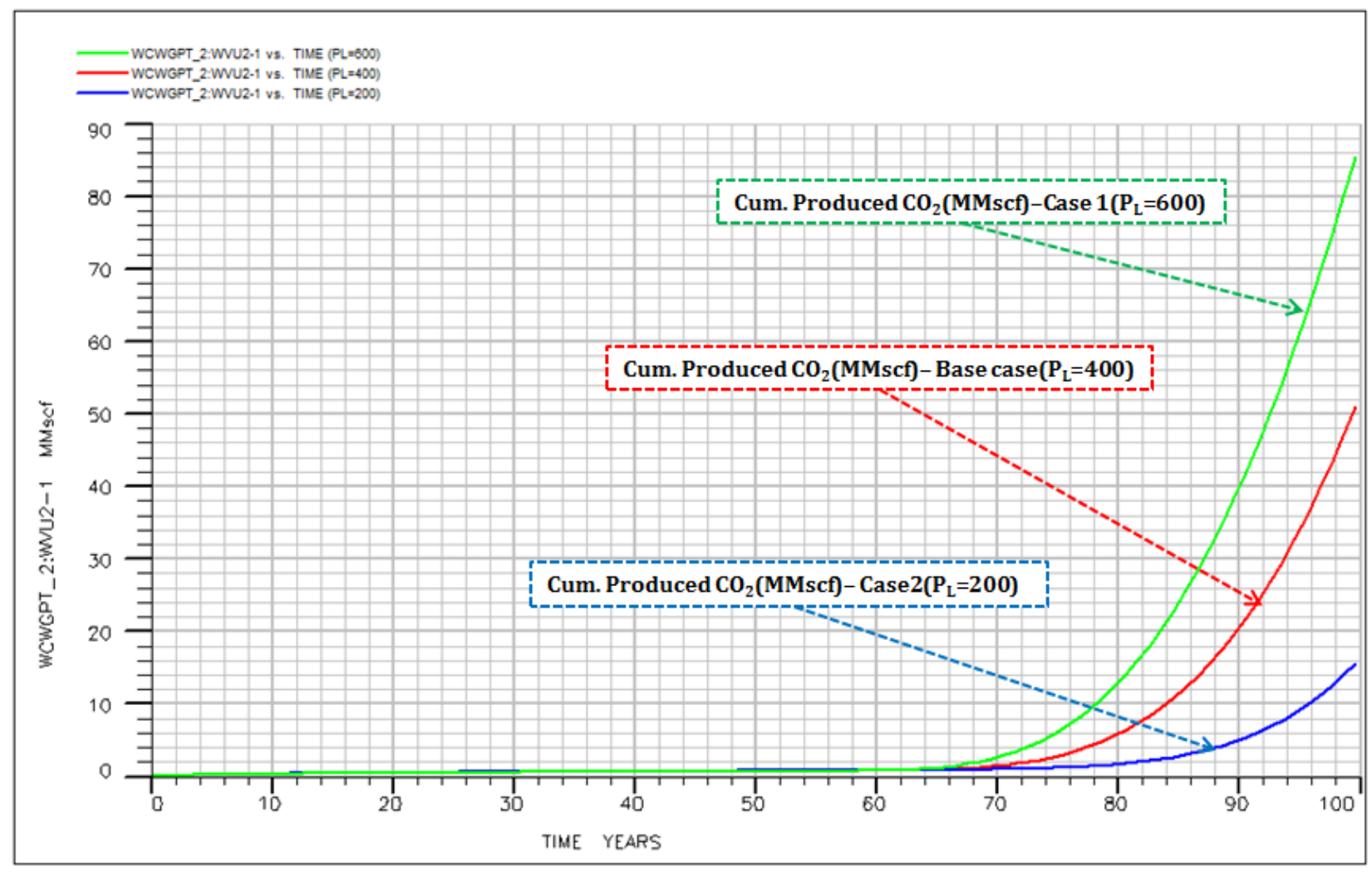

Figure 5-28.Effect of different carbon dioxide Langmuir pressure on $\mathrm{CO} 2$ breakthrough

\subsubsection{Comparison of the impact of $\mathrm{CO}_{2}$ and $\mathrm{CH}_{4}$ Isotherms on Methane Recovery, $\mathrm{CO}_{2}$ injection and production}

Figure 5-29 through Figure 5-31 illustrate the impact of increase in Langmuir pressure and volume of methane and carbon dioxide by $50 \%$ on total $\mathrm{CH} 4$ recovery, total injected $\mathrm{CO}_{2}$ and $\mathrm{CO}_{2}$ breakthrough.

According to these figures, by increasing Langmuir volume of methane by $50 \%$, the total methane recovery is enhanced by $3.4 \%$, while the $\mathrm{CO}_{2}$ injectivity is decreased by $12 \%$ and $\mathrm{CO}_{2}$ breakthrough is delayed and the resulting $\mathrm{CO}_{2}$ production has declined by $8 \%$ because of lower $\mathrm{CO}_{2}-\mathrm{CH}_{4}$ competitive adsorption capacity $(\alpha)$.

Although the increasing Langmuir volume of $\mathrm{CO}_{2}$ by $50 \%$ does not have an impact on total $\mathrm{CH}_{4}$ recovery, but it increases the total injected volume of $\mathrm{CO}_{2}$ by $18.5 \%$ and decreases the $\mathrm{CO}_{2}$ production from the offset production well by $68 \%$.

Moreover, a 50\% increase in Langmuir pressure of methane, enhance the methane recovery and total injected $\mathrm{CO}_{2}$ by $1.25 \%$ and $3.5 \%$, respectively with a sharp increase in $\mathrm{CO}_{2}$ breakthrough by $51 \%$. 
Increasing the Langmuir pressure of $\mathrm{CO}_{2}$ by $50 \%$, does not show a significant impact on $\mathrm{CH}_{4}$ recovery, while it decreases the total injected $\mathrm{CO}_{2}$ by $5 \%$ following with a substantial increase in $\mathrm{CO}_{2}$ production by $69 \%$.

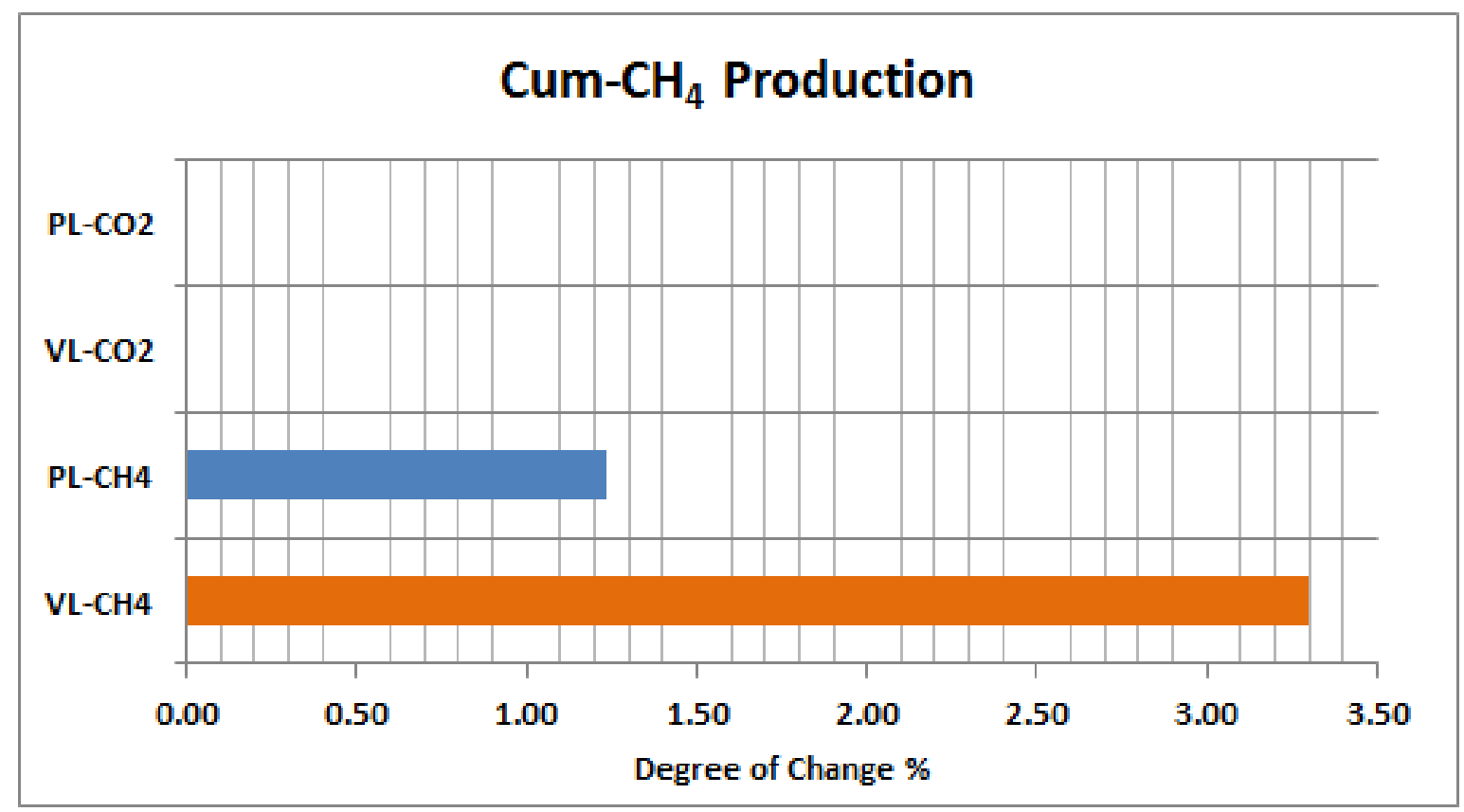

Figure 5-29. Impact of increasing $\mathrm{CH} 4$ and $\mathrm{CO} 2$ isotherms by $50 \%$ on total methane recovery

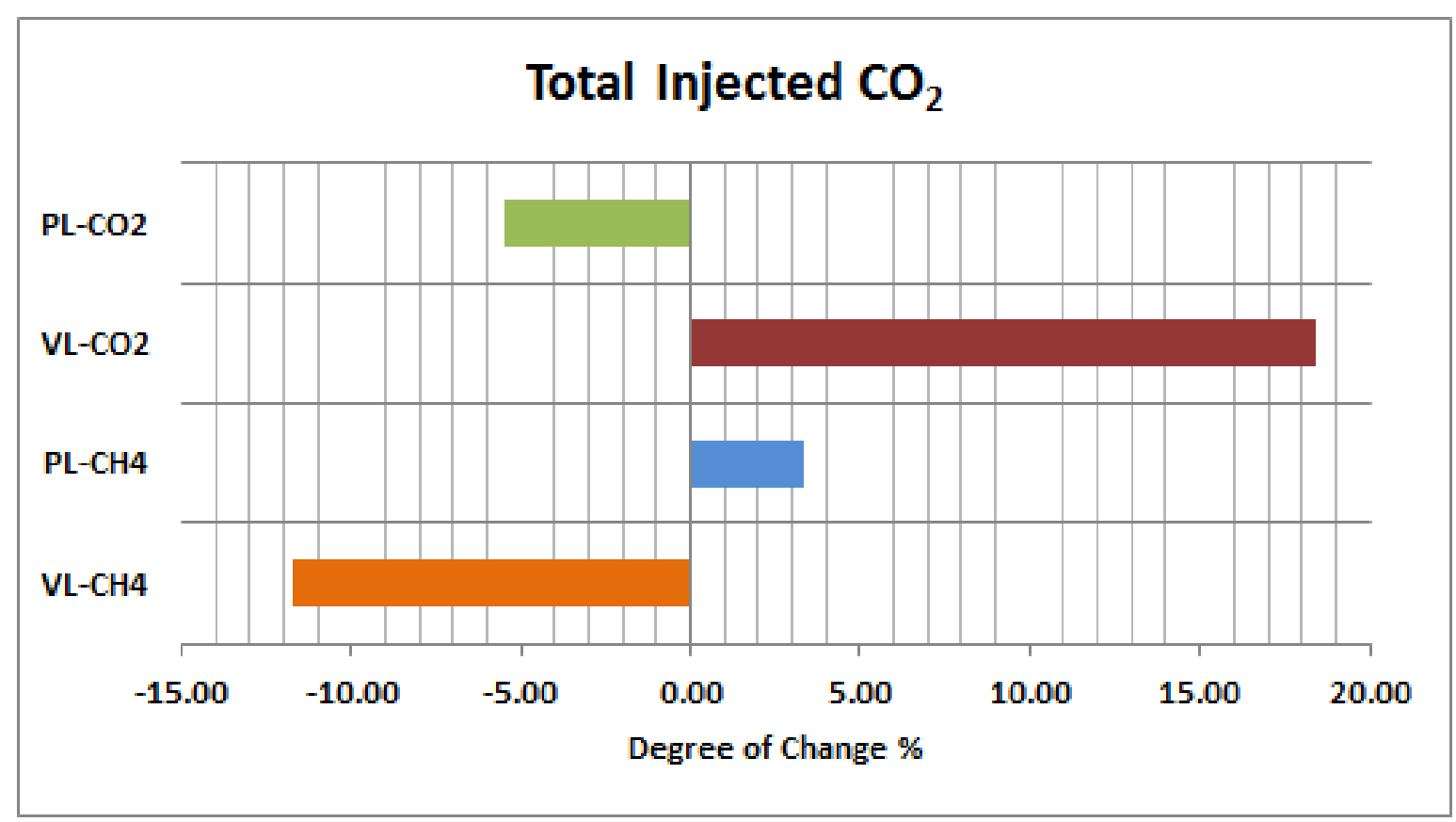

Figure 5-30. Impact of increasing $\mathrm{CH} 4$ and $\mathrm{CO} 2$ isotherms by $50 \%$ on total injected $\mathrm{CO} 2$ 


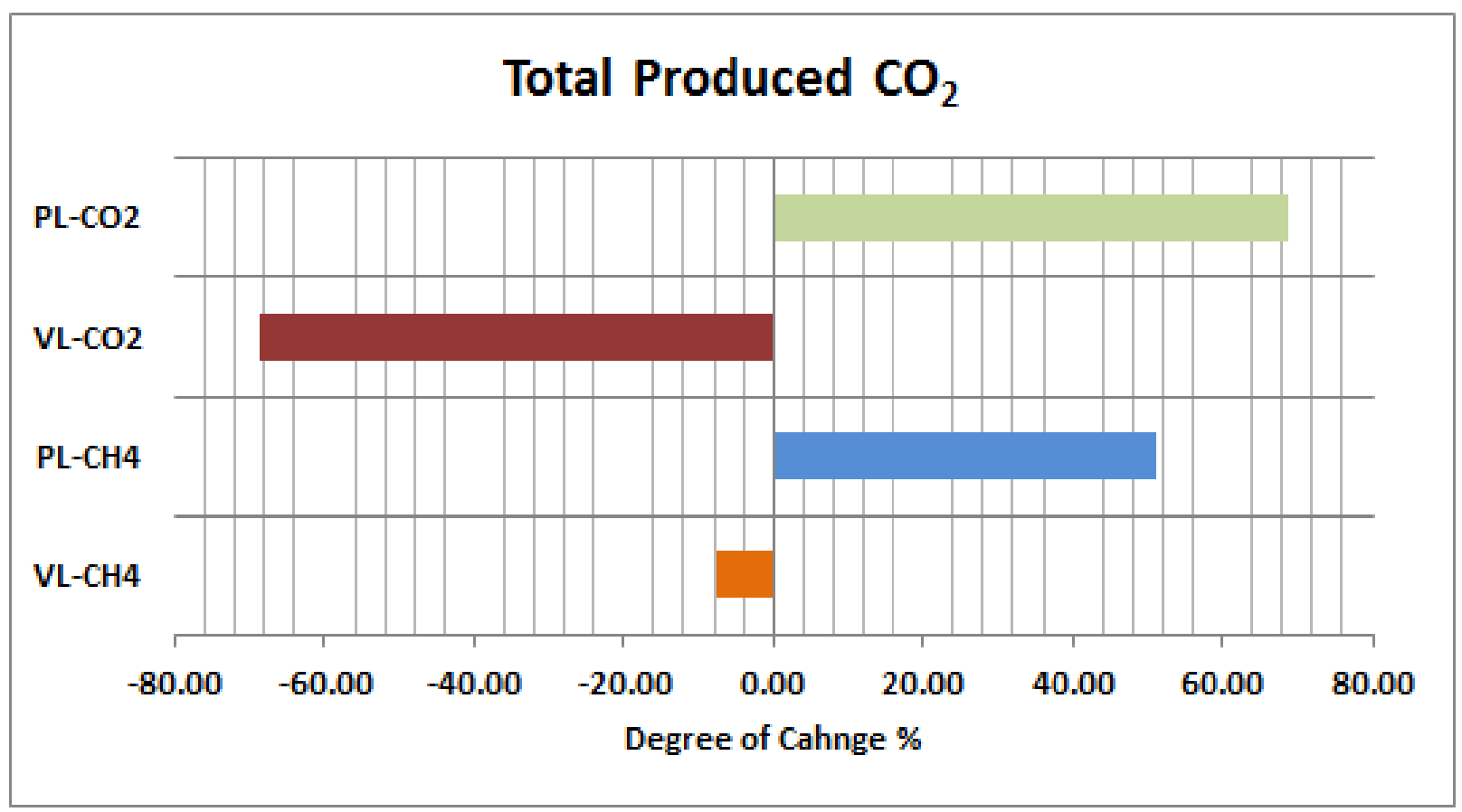

Figure 5-31. Impact of increasing $\mathrm{CH} 4$ and $\mathrm{CO} 2$ isotherms by $50 \%$ on $\mathrm{CO} 2$ breakthrough

\subsection{Summary and Conclusions}

In this chapter, comprehensive simulation studies are performed to assess the potential of shale gas reservoir for $\mathrm{CO}_{2}$ storage and to investigate the $\mathrm{CO}_{2}$ enhanced gas recovery process. Depleted history matched model is used to perform the analysis based on different injection/production patterns and different reservoir characteristic, hydraulic fracture properties and sorption features.

By designing different injection scenarios, the best practices to enhance methane recovery, minimize $\mathrm{CO}_{2}$ production while maximizing the amount of stored $\mathrm{CO}_{2}$ in shale formation are proposed. According to this study, the best injection scenario is the one with two producers and one injector in the middle with 900-970 ft spacing from the offset producing wells.

Based on our analysis, the best bottomhole injection pressure varies from 25 to $50 \%$ of lithostatic pressure to maximize $\mathrm{CO}_{2}$ storage while delaying the $\mathrm{CO}_{2}$ breakthrough time.

One of the key components of a $\mathrm{CO}_{2}$-EGR\&S process is considering the impact of sorption isotherms, which have significant impact on $\mathrm{CO}_{2}$ injectivity, $\mathrm{CO}_{2}$ breakthrough time and methane recovery. 


\section{CHAPTER 6 - DATA-DRIVEN SHALE PROXY MODEL AT HYDRAULIC FRACTURE CLUSTER LEVEL - $\mathrm{CO}_{2}$ INJECTION/STORAGE PHASE}

\subsection{Summary}

As was explained in the previous chapter, the first step in the process of modeling of $\mathrm{CO}_{2}$ injection is to evaluate a large range of injection scenarios and then, based on the results of that modeling effort, propose an appropriate set of injection scenarios. The most appropriate injection scenarios attempt to maximize $\mathrm{CH}_{4}$ production while delays $\mathrm{CO}_{2}$ breakthrough time (BT) and maximizing the amount of $\mathrm{CO}_{2}$ that is stored.

In order to perform a comprehensive uncertainty analysis, a large number of simulation runs are required. Designing and running the simulation cases to model Enhanced Gas recovery and Storage in the shale by applying Explicit Hydraulic Fracture modeling technique (EHF), is long and laborious and its implementation is computationally expensive.

In this chapter, a data driven proxy model is developed, which is capable of accurately reproducing the injection and production profiles at each cluster/stage and well, therefore making it possible to perform detailed uncertainty and optimization analysis of Enhanced Gas Recovery (EGR) and $\mathrm{CO}_{2}$ Storage process. The detailed procedure for development of the data-driven proxy model is explained .The proxy model is validated using blind simulation runs.

\subsection{Methodology}

The starting point and the most important step toward building a data driven shale proxy model for $\mathrm{CO}_{2}$ driven Enhanced Gas Recovery and Storage $\left(\mathrm{CO}_{2}\right.$-EGR\&S), is the comprehensive spatio-temporal database generation by designing few simulation runs and considering the uncertainty domain and operational limitations.

The $\mathrm{CO}_{2}$ injection strategy includes four cases with different distances between injection and production well(s). History matched horizontal laterals in WVU pad are used for this study (Please refer to Chapter 5 for more details).

Before starting the injection process all the wells in each of the cases, are put on production for 100 years. When the production well, which is going to be converted to an injection well, produced $75 \%$ of its accessible gas, the $\mathrm{CO}_{2}$ injection starts and continues until the end of the $\mathrm{t}=100$ years. 
Five simulation runs are defined for each case (Totally 20 runs) to cover the desired range of reservoir characteristics and different operational constraints.

The generated comprehensive spatio-temporal database, is used to train and validate a multilayer feedforward back-propagation neural networks to accurately mimic the reservoir simulator behavior and regenerate $\mathrm{CH} 4$ production and $\mathrm{CO}_{2}$ injection profiles and also predicting $\mathrm{CO}_{2}$ breakthrough time and $\mathrm{CO}_{2}$ Production profile for each cluster of hydraulic fracture in the production and injection well(s).

In order to make the validation one-step furthered, the developed Data-driven proxies are validated with a set of completely blind simulation runs that are not used during the training process. The detailed workflow for Data-driven shale proxy model development for $\mathrm{CO}_{2}$-EGR\& $\mathrm{S}$ process is shown in Figure 6-1.

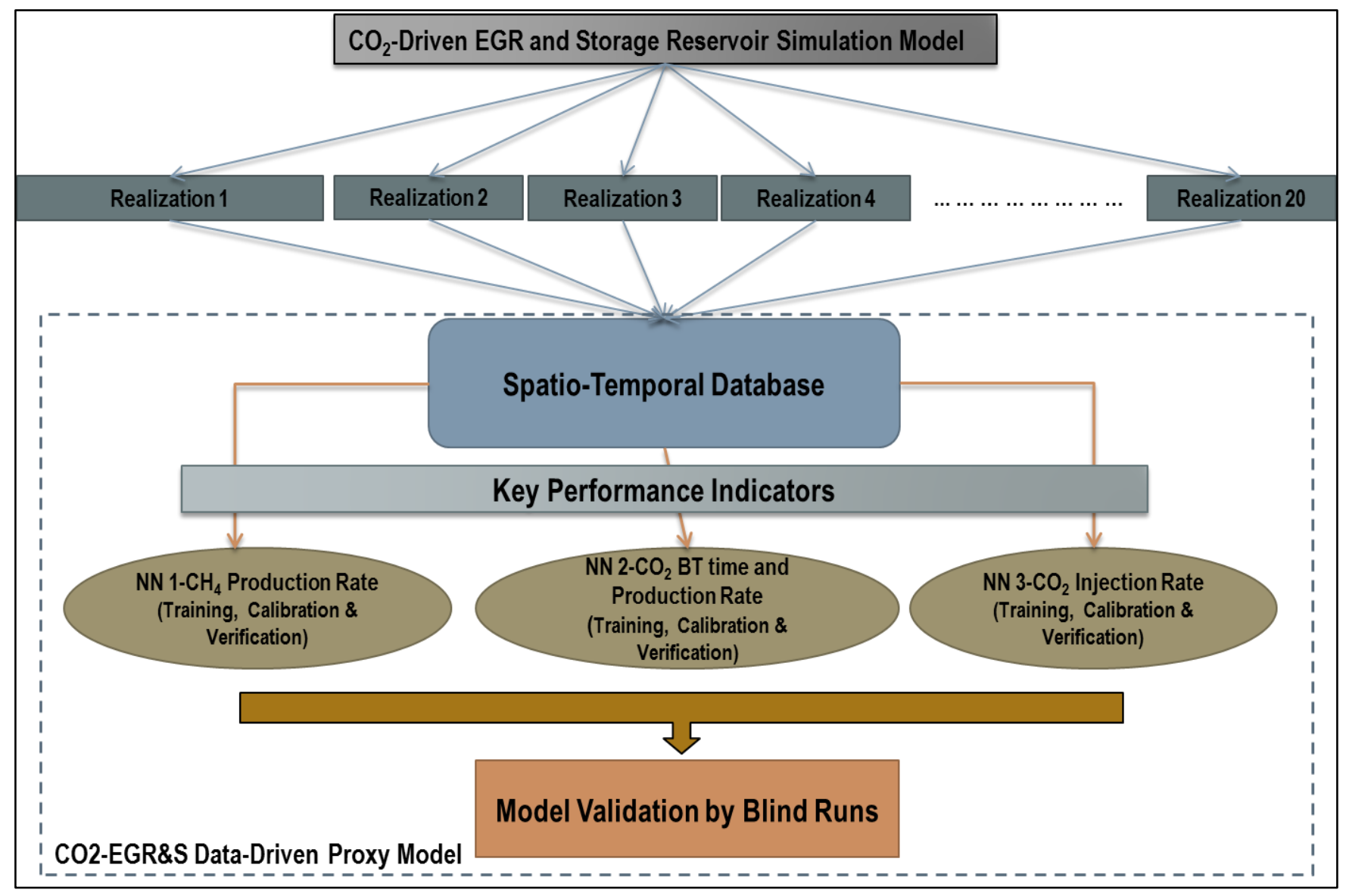

Figure 6-1. CO2-EGR\&SData-driven Shale proxy model development workflow 


\subsection{Spatio-Temporal Database Generation}

As briefly stated before, the most important step in the development of any data-centric reservoir model is the assimilation of a representative spatio-temporal database, which forms the foundation of Data-driven shale proxy model for $\mathrm{CO}_{2}$-EGR\&S process. The extent at which this database actually represents the flow and storage behavior of the shale reservoir that is being modeled, determines the potential degree of success in developing a successful data-driven shale proxy model.

Figure 6-2 illustrates the production injection patterns in four cases. For each pattern (or case), five simulation runs are defined to cover the desired range of reservoir characteristics (i.e. Matrix, natural fracture and hydraulic fracture properties as well as different Langmuir isotherms) and different operational constraints (Flowing bottom-hole pressure and Bottom-hole injection pressure).

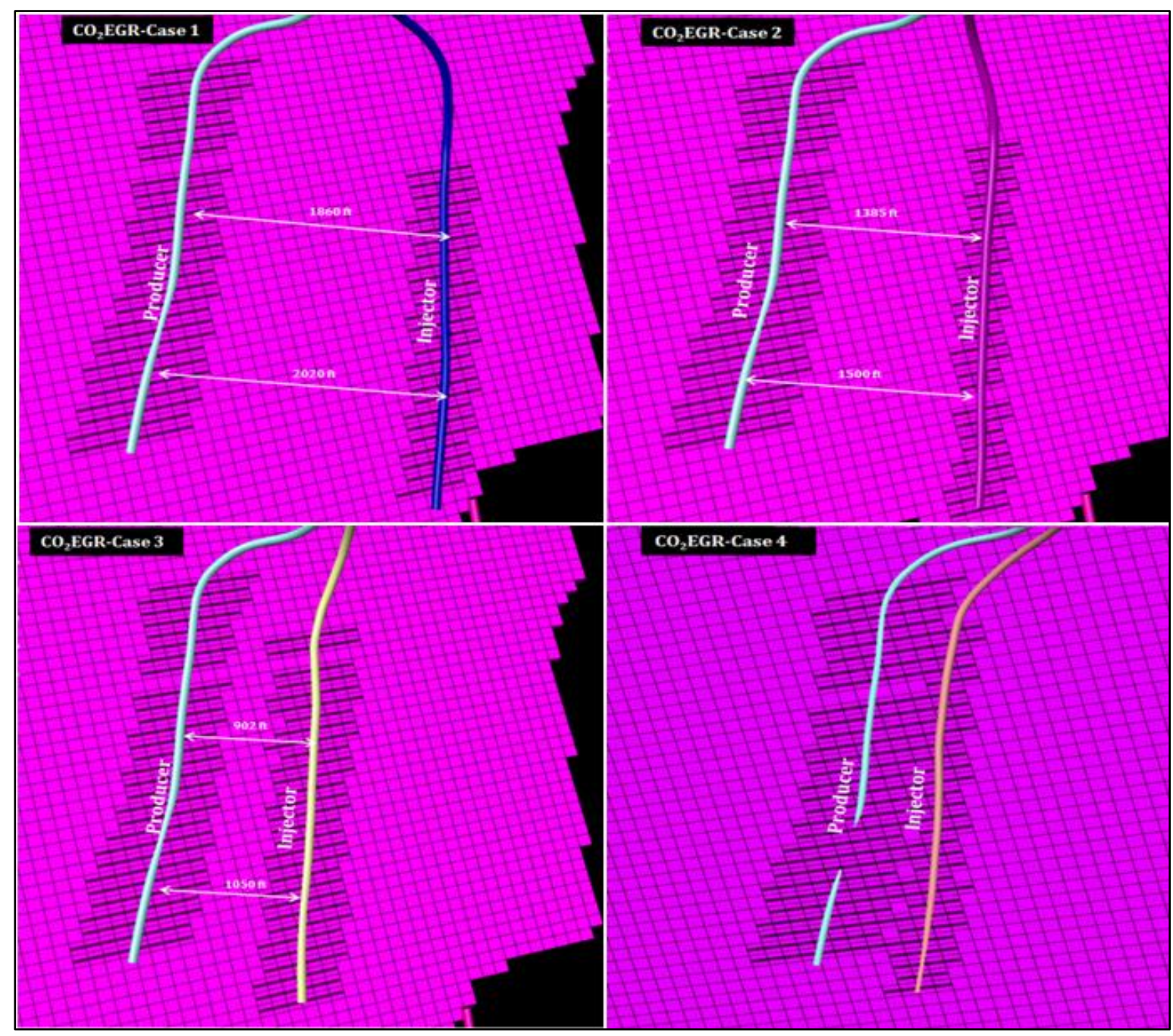

Figure 6-2. $\mathrm{CO}_{2}$-EGR\&S Process Simulation Cases (Producer and Injector patterns) 
In the first three proposed simulation cases, the average distance between producer and injector varies from 2020 to $900 \mathrm{ft}$. In the Case 4, the hydraulic fractures for both production and injection laterals are overlapped and therefore, the early breakthrough can be expected for this pattern. This case is included in the spatio-temporal database to make sure that the developed Data-driven proxy model is robust enough to predict the production and injection performance in any situation.

This database includes 116,000 pairs of input-output that is used for the training process. The inputs are static data (e.g. reservoir and hydraulic fracture characteristics, sorption features etc.) and operational constraint and the output are $\mathrm{CH}_{4}$ production and $\mathrm{CO}_{2}$ injection profiles and also $\mathrm{CO}_{2}$ breakthrough time and $\mathrm{CO}_{2}$ Production profile for each cluster of hydraulic fracture in the production and injection well(s).

A complete list of inputs and corresponding ranges that are included in the data set is shown in Table 6-1.

Table 6-1.Main input data for $\mathrm{CO}_{2}$-EGR\&S Data-driven shale proxy models development

\begin{tabular}{|c|c|c|c|c|}
\hline $\begin{array}{c}\text { Matrix porosity } \\
{[0.054-0.125]}\end{array}$ & $\begin{array}{l}\text { Matrix permeability } \\
{[0.0001-0.0008(\mathrm{md})]}\end{array}$ & $\begin{array}{c}\text { Natural fracture } \\
\text { porosity } \\
{[0.01-0.035]}\end{array}$ & $\begin{array}{c}\text { Natural fracture } \\
\text { permeability } \\
{[0.001-0.004} \\
\text { (md)] }\end{array}$ & $\begin{array}{l}\text { Sigma factor } \\
{[0.005-0.08]}\end{array}$ \\
\hline $\begin{array}{l}\text { Hydraulic fracture height } \\
\qquad[100-125 \mathrm{ft}]\end{array}$ & $\begin{array}{c}\text { Hydraulic fracture } \\
\text { length } \\
{[200-1100 \mathrm{ft}]}\end{array}$ & $\begin{array}{c}\text { Hydraulic fracture } \\
\text { conductivity } \\
{[0.1-5.4(\mathrm{md}-\mathrm{ft})]}\end{array}$ & $\begin{array}{l}\text { Net pay thickness } \\
{[113-128(\mathrm{ft})]}\end{array}$ & $\begin{array}{l}\mathrm{CH}_{4} \text {-Longmuir } \\
\text { volume } \\
{[55-91(\mathrm{scf} / \text { ton })]}\end{array}$ \\
\hline $\begin{array}{c}\mathrm{CH}_{4} \text {-Longmuir pressure } \\
{[600-790 \mathrm{psi}]}\end{array}$ & $\begin{array}{c}\mathrm{CO}_{2} \text {-Longmuir volume } \\
{[70-120(\mathrm{scf} / \text { ton })]}\end{array}$ & $\begin{array}{c}\mathrm{CO}_{2} \text {-Longmuir } \\
\text { pressure } \\
{[400-580 \mathrm{psi}]}\end{array}$ & $\begin{array}{c}\mathrm{CH}_{4} \text {-Diffusion } \\
\text { coefficient } \\
{[0.2-4(\mathrm{ft} 2 / \text { day })]}\end{array}$ & $\begin{array}{c}\mathrm{CO}_{2} \text {-Diffusion } \\
\text { coefficient } \\
{[1-20(\mathrm{ft} 2 / \text { day })]}\end{array}$ \\
\hline $\begin{array}{l}\text { Bottom-hole Injection } \\
\text { Pressure[1680-3360 psi] }\end{array}$ & $\begin{array}{l}\text { Flowing Bottom-hole } \\
\text { Pressure[820-130 psi] }\end{array}$ & & & \\
\hline
\end{tabular}

In order to take into account the impact of different grid blocks properties on each cluster production/injection "Tier system" is defined.

Therefore, three different tiers are defined and a property for each tier is calculated by averaging the properties of all the grid blocks in the corresponding tier. Moreover, in order to teach the NNs, the interference effect between the clusters, they are divided into four classes based on their relative location respect to the other offset ones. More details about the Tier system and cluster type definition and can be found in Chapter 4 of this dissertation. 


\subsection{Data-Driven $\mathrm{CO}_{2}$-EGR\&S Proxy Development for $\mathrm{CH}_{4}$ Production Rate, $\mathrm{CO}_{2}$ Injection Rate and $\mathrm{CO}_{2}$ Breakthrough}

In this section, the results of four Data-driven $\mathrm{CO}_{2}$-EGR\&S proxy models at hydraulic fracture cluster level are presented. The Data-driven proxies are developed to predict: a) $\mathrm{CH}_{4}$ production rate, b) $\mathrm{CO}_{2}$ injection rate , c) $\mathrm{CO}_{2}$ Breakthrough time and d) $\mathrm{CO}_{2}$ production rate for each hydraulic fracture clusters as wells as corresponding laterals. The summation of all the rates from all clusters generates production/injection profile for the corresponding horizontal lateral.

\subsection{1 $\quad \mathrm{CH}_{4}$ Production Profile Predication}

The first Data-driven $\mathrm{CO}_{2}$-EGR\&S proxy model is developed to re-generated annual methane production rate for each cluster of hydraulic fractures for 100 years, which is already generated by Eclipse reservoir simulator. Twenty simulation runs are designed and run based on four Injector/Producer patterns to generate 1160 unique methane production profiles with different reservoir characteristics, hydraulic fracture properties, sorption features (sorption time and Langmuir isotherms) and operational constraints (56 clusters* 5 runs (Run 1 to5 -Case1) +58 clusters* 5 runs (Run 6 to 10-Case2) +59 clusters* 5 runs (Run 11to15-Case3) +59 clusters* 5 runs (Run 16 to 20 -Case4) $=1160)$.

The generated database with 116,000 pairs of input-output is then used to train a multilayer feed-forward back-propagation neural network. Before starting the training and validation process, fuzzy pattern recognition technique is used to determine the degree of influence of all input parameters on the production indicator (the output: the target of the correlation).

Figure 6-3 illustrated the KPI analysis results for $\mathrm{CH}_{4}$ production. According to this plot, the most influential parameters on methane production are duration of production, operational constraints, producer/injector patterns and relative location of clusters.

The second most influential parameters are those, which control the reserve such as isotherms, net pay thickness natural fracture porosity and matrix porosity.

Some other parameters such as hydraulic fracture components, natural fracture properties that control the accessibility of gas to the clusters can be considered as a third group of parameters that has high influence on the production. Although, they are essential in the productivity of the shale gas wells at the beginning of the production, but for long-term production performance, operational constraints in addition to those parameters that control the reserve, play more important role. 


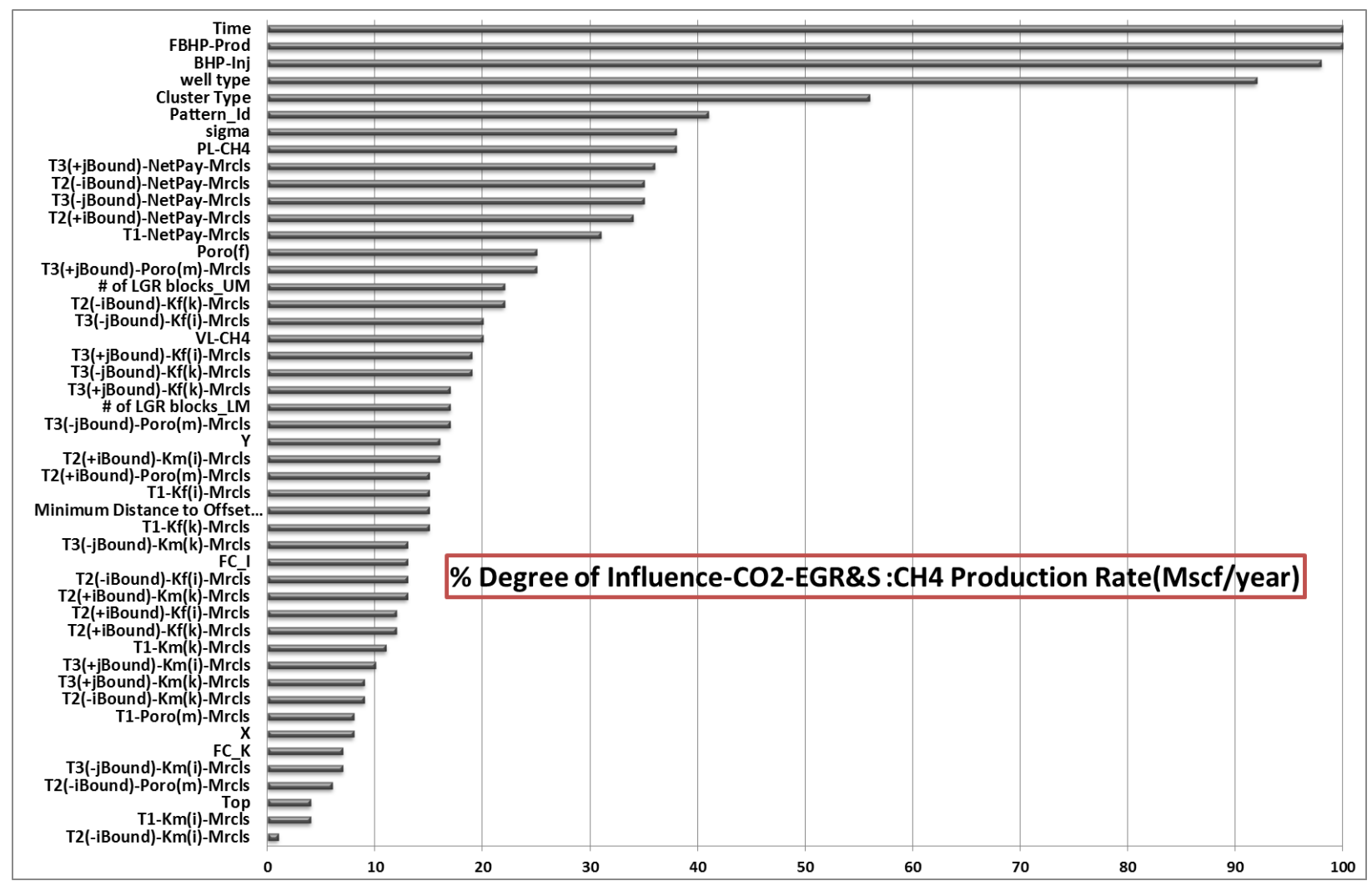

Figure 6-3. Key performance indicators results based on the database for CO2-EGR\&S-Methane production rate (Mscf/year)

To continue with the training and validation procedure, the neural network architecture is designed to have one hidden layer with 55 hidden neurons that are selected based on the number of data records available and the number of input parameters selected in each training process. In the training process, the data set is partitioned into three separate segments by using intelligent partitioning technique. Data is partitioned with $80 \%$ training fraction, $20 \%$ for calibration and verification (10\% for each).

The training and validation process can be started with maximum number of inputs and based on the modeler's reservoir engineering judgments and also the training results, this number can be reduced to the optimum numbers of inputs to obtain the best training-calibration and verification results. The table summarizes the list of optimum inputs (47 inputs) that are used for development of the Data-driven $\mathrm{CO}_{2}$ EGR\&S proxy model to generate $\mathrm{CH}_{4}$ annual production profile.

The cross plots for predicted and simulated values of annual methane production rate (Mscf/year) for training, calibration and verification steps (from left to right) are shown in Figure 6-4. In these plots, $x$ axis corresponds to the neural network predicted gas rate and the y-axis shows the simulated gas rate by Eclipse. 
(Annual CH4 Production Rate)

\begin{tabular}{|c|c|c|}
\hline \multicolumn{3}{|c|}{ CH4 Production NN- Input Parameters } \\
\hline \multirow{30}{*}{ Static Inputs } & \multirow{21}{*}{$\begin{array}{c}\text { Cluster/Reservoir } \\
\text { Characteristics }\end{array}$} & Location $(X, Y)$ \\
\hline & & Min. Distance to Offset Injector-Producer(Fracture edge) \\
\hline & & Top \\
\hline & & Cluster Type \\
\hline & & T1-Km(I,K)-Marcellus \\
\hline & & T2(-iBound)-Km(I,K)-Marcellus \\
\hline & & $\mathrm{T} 2(+\mathrm{iBound})-\mathrm{Km}(\mathrm{I}, \mathrm{K})$-Marcellus \\
\hline & & T3(-jBound)-Km(I,K)-Marcellus \\
\hline & & T3(-jBound)-Km(I,K)-Marcellus \\
\hline & & T1-Porosity-Marcellus \\
\hline & & T2(-iBound)-Porosity-Marcellus \\
\hline & & T2(+iBound)-Porosity-Marcellus \\
\hline & & T3(-jBound)-Porosity-Marcellus \\
\hline & & T3(-jBound)-Porosity-Marcellus \\
\hline & & T1-Net Pay-Marcellus \\
\hline & & T2(-iBound)-Net Pay-Marcellus \\
\hline & & T2(+iBound)-Net Pay-Marcellus \\
\hline & & T3(-jBound)-Net Pay-Marcellus \\
\hline & & T3(-jBound)-Net Pay-Marcellus \\
\hline & & CH4-Langmuire Pressure \\
\hline & & CH4-Langmuire Volume \\
\hline & \multirow{2}{*}{ HF Properties } & Fracture Conductivity in I,K \\
\hline & & Hydraulic Fracture half length \\
\hline & \multirow{7}{*}{$\begin{array}{l}\text { Natural Fracture } \\
\text { Properties }\end{array}$} & T1-Kf(I,k)-Marcellus \\
\hline & & T2(-iBound)-Kf(I,k)-Marcellus \\
\hline & & T2(+iBound)-Kf(I,k)-Marcellus \\
\hline & & T3(-jBound)-Kf(I,k)-Marcellus \\
\hline & & T3(-jBound)-Kf(I,k)-Marcellus \\
\hline & & Sigma \\
\hline & & Porosity \\
\hline \multirow{3}{*}{ Dynamic Inputs } & \multicolumn{2}{|r|}{ Time } \\
\hline & \multicolumn{2}{|r|}{ Flowing Bottomhole Pressure } \\
\hline & \multicolumn{2}{|r|}{ Bottomhole Injection Pressure } \\
\hline \multirow{2}{*}{ Additional Inputs } & \multicolumn{2}{|r|}{ Pattern ID } \\
\hline & \multicolumn{2}{|r|}{ Well type } \\
\hline \multicolumn{3}{|r|}{47 Inputs } \\
\hline
\end{tabular}

The result with an $\mathrm{R}^{2}$ of more than 0.99 in all steps shows the successful development of Data-driven shale proxy model for $\mathrm{CH}_{4}$ production profile prediction.

Figure 6-5 and Figure 6-6 show some examples of the comparison of reservoir simulation output for the annual methane production rate during $\mathrm{CO}_{2}$-EGR\&S process with the predicted one by Data-driven proxy model for some of the clusters as well as some of the laterals for all four production and injection patterns/cases. The rest of the results are shown in Appendix B. 


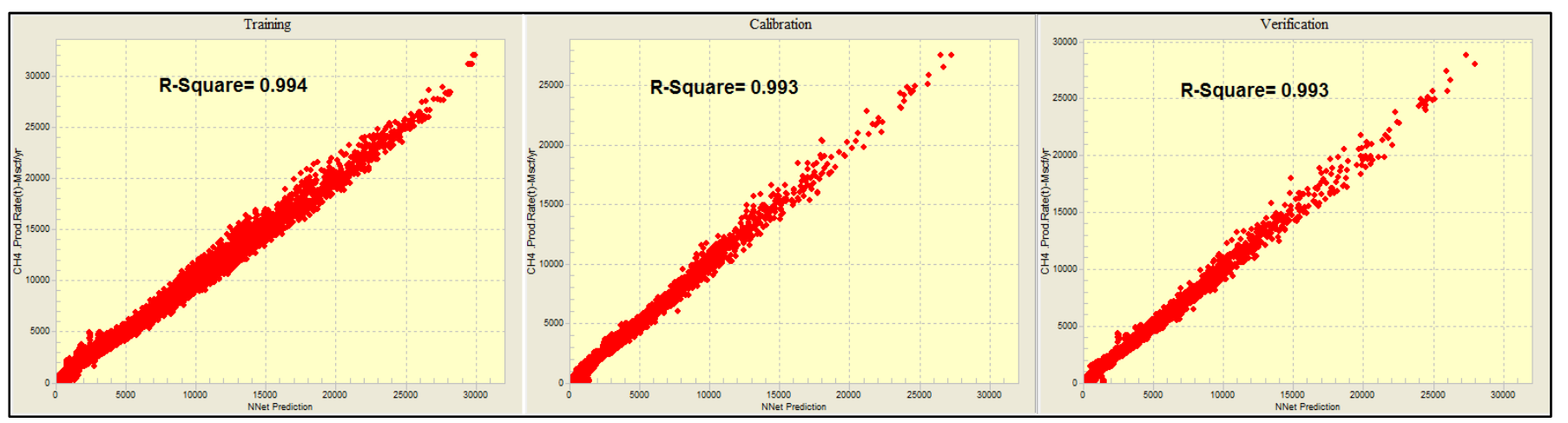

Figure 6-4. NN training, calibration and verification results (From left to right relatively) - CH4 Production (Mscf/year)

It should be noted that, Data-driven proxy model, AI-based proxy model and Cluster based surrogate reservoir model (CSRM) are used in this dissertation interchangeably.

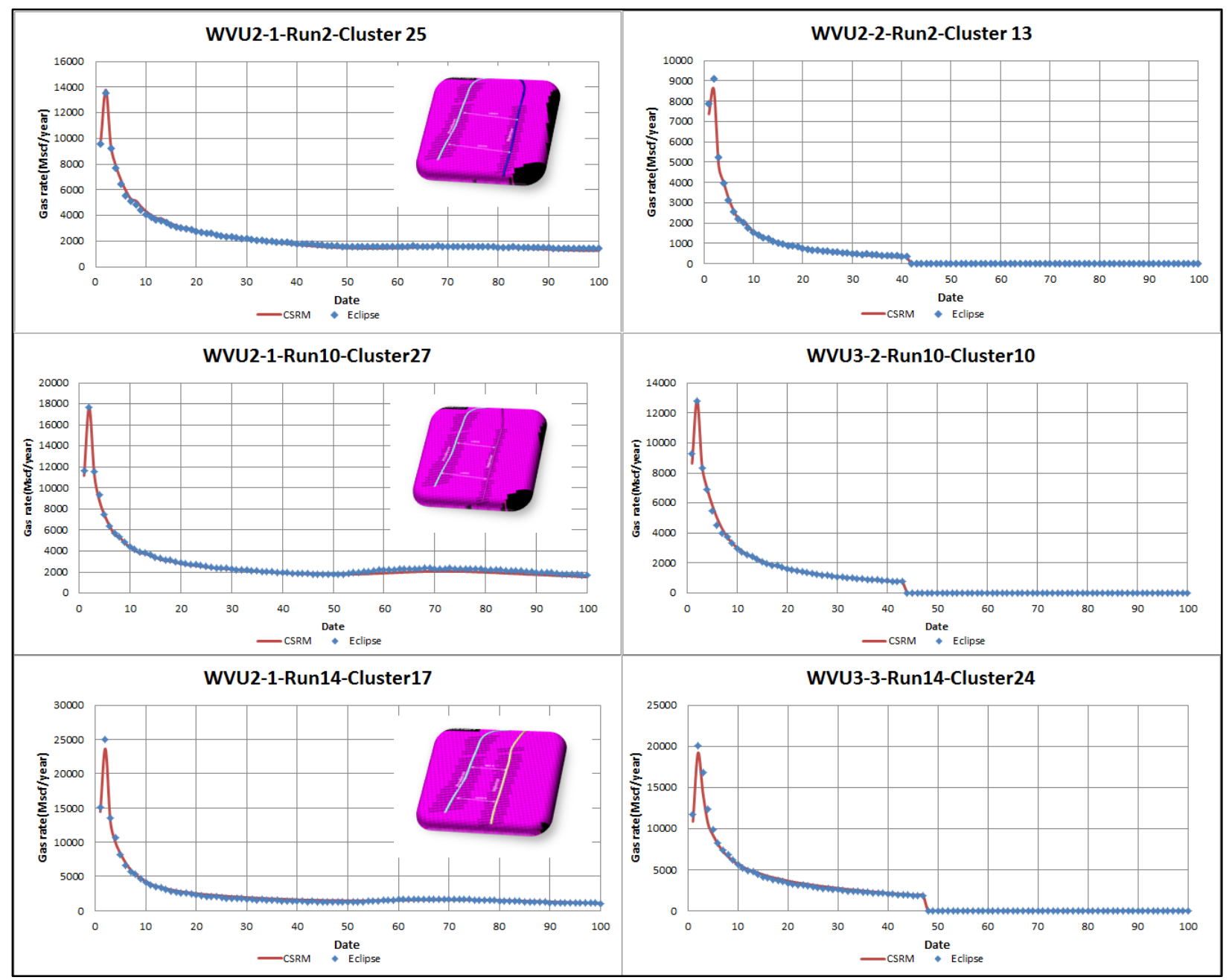

Figure 6-5.Comparison of $\mathrm{CH}_{4}$ production profile (Mscf/year) from simulator and CO2-EGR\&S Data-driven proxy model for some of the clusters (Cases1, 2 and 3) 


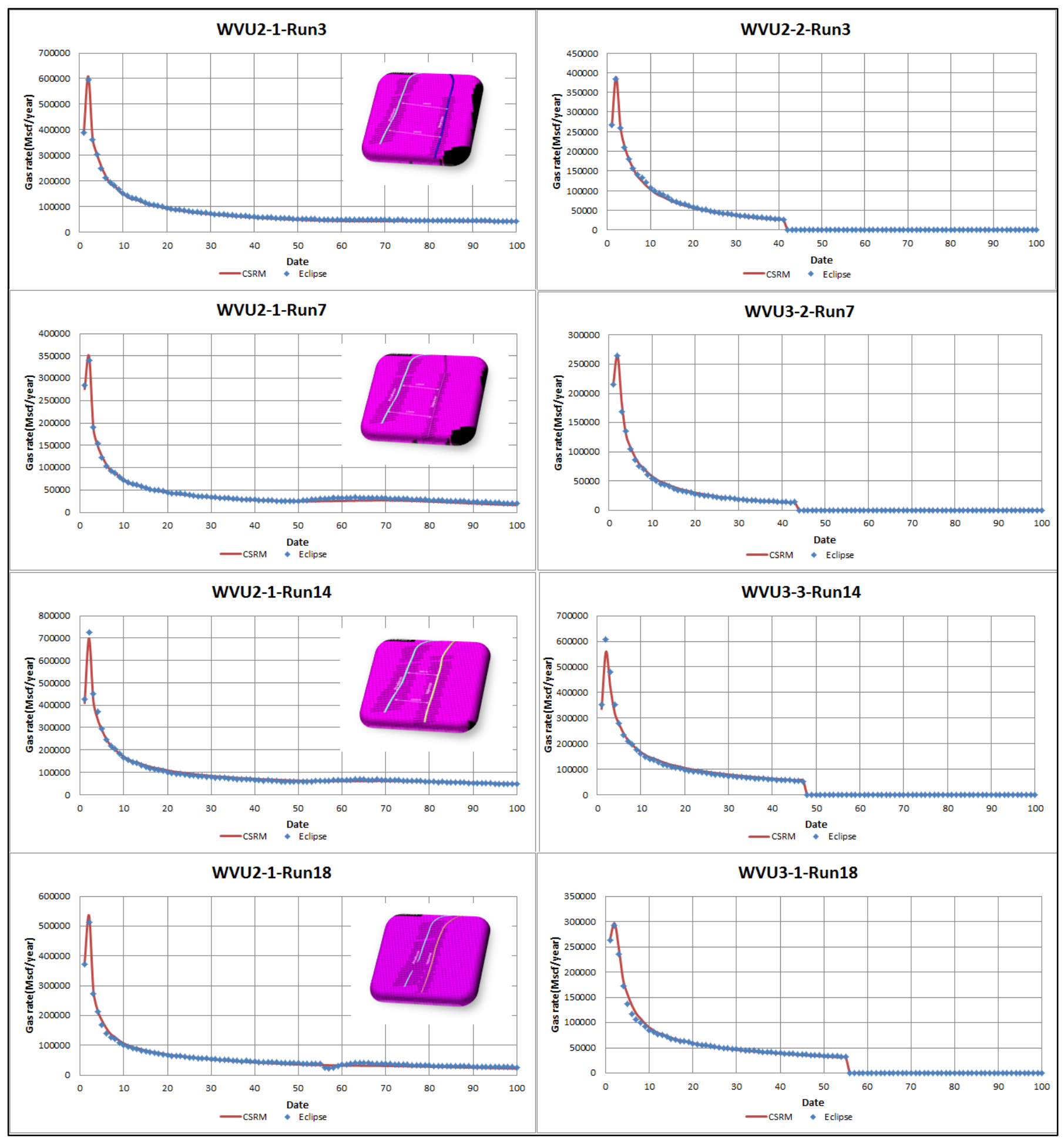

Figure 6-6.Comparison of $\mathrm{CH}_{4}$ production profile (Mscf/year) from simulator and CO2-EGR\&S Data-driven proxy model for some of the laterals (Cases1, 2, 3 and 4) 
In all the plots blue dots represent the annual methane production rate generated by Eclipse and the solid red line is the result of Data-driven $\mathrm{CO}_{2}$-EGR\&S proxy model. The results are self-descriptive enough to show the capability of Data-driven proxy model in predicting the $\mathrm{CH}_{4}$ production profile at hydraulic fracture cluster, and lateral level for different production/injection patterns.

In all four cases, WVU2-1 (the left lateral in Figure 6-5 and Figure 6-6) continue methane production for 100 years while the $\mathrm{CH}_{4}$ production from WVU2-2(Case2), WVU3-2(Case2), WVU3-3(Case3) and WVU3-1(Case4) is stopped after producing the $75 \%$ of their accessible gas and then they converted to an $\mathrm{CO}_{2}$ injection well.

The hump in methane production profile in WVU2-1 well can be observed when the displaced methane by $\mathrm{CO}_{2}$ (during the counter diffusion process) reaches the producting well after 8-20 years, depends on the distance, reservoir characteristics and bottom-hole injection pressure.

\subsection{2 $\mathrm{CO}_{2}$ Injection Profile Predication}

The second Data-driven $\mathrm{CO}_{2}$-EGR\&S proxy model is developed to mimic the numerical simulation behavior and re-generate the annul $\mathrm{CO}_{2}$ injection rate (Mscf/year) from the injection startup date until $\mathrm{t}=100$ years. The same procedure for building the first proxy $\left(\mathrm{CH}_{4}\right.$ production $)$ is followed here as well.

Table 6-3 summarizes the list of optimum inputs (50 inputs) that are used for development of the Datadriven $\mathrm{CO}_{2}$-EGR\&S proxy model to generate $\mathrm{CO}_{2}$ injection profile (Mscf/year).

The representative database with 116, 000 pairs of input-output is used to identify the critical parameters and their degree of influence on $\mathrm{CO}_{2}$ injectivity in the shale formation (Figure 6-7). As can be seen from this chart, starting time of injection, operational constraints and the inj./prod. configuration and relative location of clusters are top ranked parameters during $\mathrm{CO}_{2}$ injection process.

$\mathrm{CO}_{2} / \mathrm{CH}_{4}$ Langmuir isotherms, fracture porosity, net pay and fracture half-length are the next top influential parameters for long-term $\mathrm{CO}_{2}$ injection (44 to 58 years).

Having the hydraulic fracture conductivity as low ranked parameter does not necessarily diminish their importance during the $\mathrm{CO}_{2}$ injection process. It should be noted that, hydraulic fracture properties are critical components to allow $\mathrm{CO}_{2}$ injection in nano-Darcy permeability shale to be initiated. When the injection is started and last for many years, then the other parameters come into the picture and show their contribution for long time injection practice. 


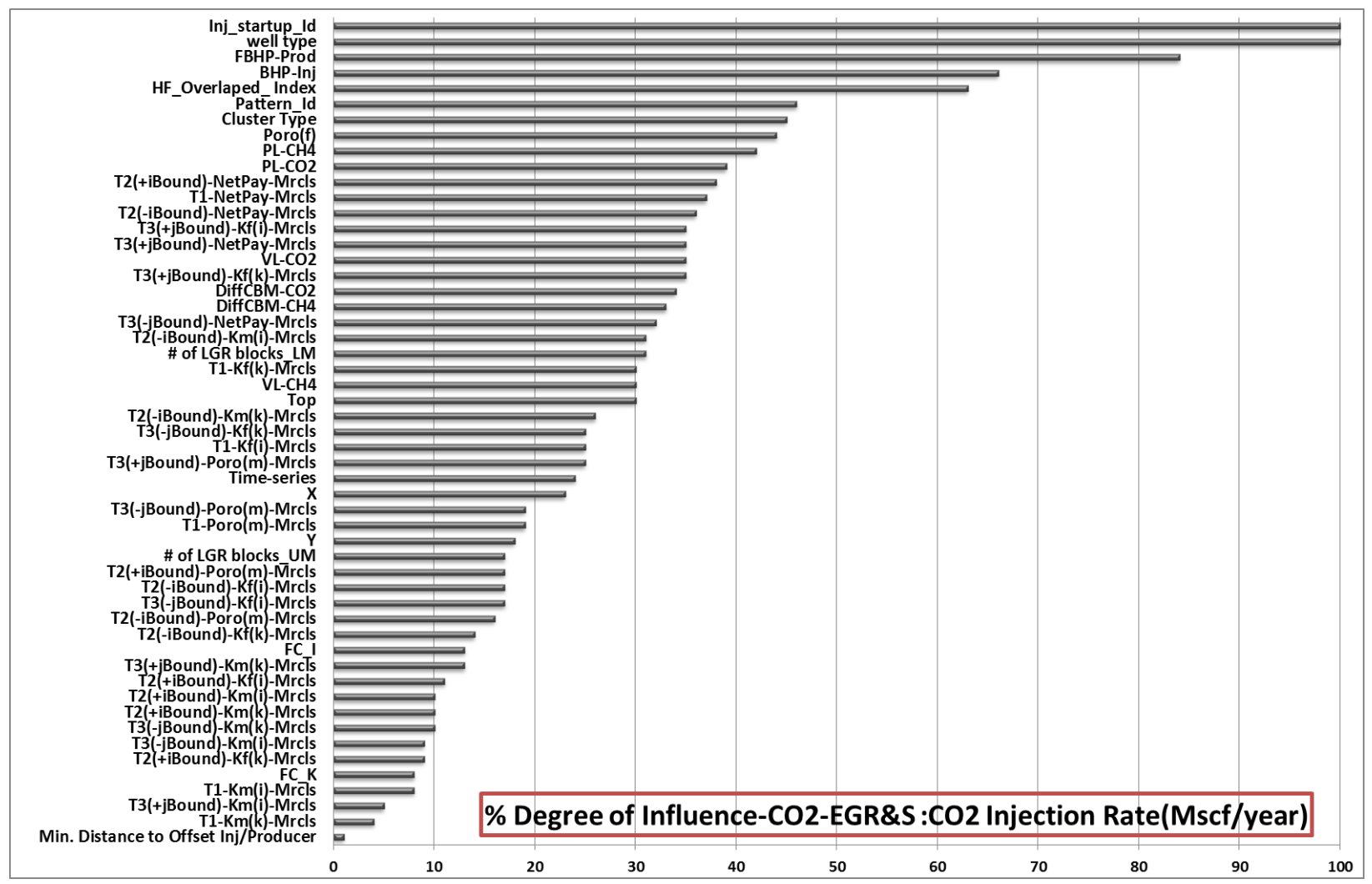

Figure 6-7. Key performance indicators results based on the database for CO2-EGR\&S-CO2 Injection rate (Mscf/year)

By having the better understanding of the key parameters during $\mathrm{CO}_{2}$ injection process, the spatiotemporal database is partitioned with $80 \%$ training, and $20 \%$ for calibration and verification. The designed neural network has one hidden layers with 58 hidden neurons.

The cross plots for predicted and simulated values of $\mathrm{CO}_{2}$ injection rate (Mscf/year) for training, calibration and verification steps (from left to right) are shown in Figure 6-8. In these plots, x-axis corresponds to the neural network predicted $\mathrm{CO}_{2}$ injection rate (Mscf/year) and the y-axis shows the simulated $\mathrm{CO}_{2}$ injection rate by Eclipse.

The data driven $\mathrm{CO}_{2}$-EGR\&S proxy model is successfully developed to predict $\mathrm{CO}_{2}$ injection rate (Mscf/year) with the $\mathrm{R}^{2}$ of more than 0.99 in all steps (Training, Calibration and verification). 


\begin{tabular}{|c|c|c|}
\hline \multicolumn{3}{|c|}{ CO2 Injection NN- Input Parameters } \\
\hline \multirow{29}{*}{ Static Inputs } & \multirow{21}{*}{$\begin{array}{c}\text { Cluster/Reservoir } \\
\text { Characteristics }\end{array}$} & Location $(X, Y)$ \\
\hline & & Min. Distance to Offset Injector-Producer(Fracture edge) \\
\hline & & Top \\
\hline & & Cluster Type \\
\hline & & T1-Km(I,K)-Marcellus \\
\hline & & T2(-iBound)-Km(I,K)-Marcellus \\
\hline & & $\mathrm{T} 2(+\mathrm{iBound})-\mathrm{Km}(\mathrm{I}, \mathrm{K})$-Marcellus \\
\hline & & T3(-jBound)-Km(I,K)-Marcellus \\
\hline & & T3(-jBound)-Km(I,K)-Marcellus \\
\hline & & T1-Porosity-Marcellus \\
\hline & & T2(-iBound)-Porosity-Marcellus \\
\hline & & T2(+iBound)-Porosity-Marcellus \\
\hline & & T3(-jBound)-Porosity-Marcellus \\
\hline & & T3(-jBound)-Porosity-Marcellus \\
\hline & & T1-Net Pay-Marcellus \\
\hline & & T2(-iBound)-Net Pay-Marcellus \\
\hline & & T2(+iBound)-Net Pay-Marcellus \\
\hline & & T3(-jBound)-Net Pay-Marcellus \\
\hline & & T3(-jBound)-Net Pay-Marcellus \\
\hline & & CH4/CO2-Langmuire Pressure \\
\hline & & $\mathrm{CH} 4 / \mathrm{CO} 2$-Langmuire Volume \\
\hline & \multirow{2}{*}{ HF Properties } & Fracture Conductivity in $\mathrm{I}, \mathrm{K}$ \\
\hline & & Hydraulic Fracture half length \\
\hline & \multirow{6}{*}{$\begin{array}{l}\text { Natural Fracture } \\
\text { Properties }\end{array}$} & T1-Kf(I,k)-Marcellus \\
\hline & & T2(-iBound)-Kf(I,k)-Marcellus \\
\hline & & T2(+iBound)-Kf(I,K)-Marcellus \\
\hline & & T3(-jBound)-Kf(I,k)-Marcellus \\
\hline & & $T 3(-j B o u n d)-K f(I, k)-$ Marcellus \\
\hline & & Porosity \\
\hline \multirow{3}{*}{ Dynamic Inputs } & \multicolumn{2}{|r|}{ Time } \\
\hline & \multicolumn{2}{|r|}{ Flowing Bottomhole Pressure } \\
\hline & \multicolumn{2}{|r|}{ Bottomhole Injection Pressure } \\
\hline \multirow{3}{*}{ Additional Inputs } & \multicolumn{2}{|r|}{ Pattern ID } \\
\hline & \multicolumn{2}{|r|}{ Injection start up ID } \\
\hline & \multicolumn{2}{|r|}{ Well type } \\
\hline \multicolumn{3}{|r|}{50 Inputs } \\
\hline
\end{tabular}

Figure 6-9 and Figure 6-10 show some examples of the comparison of reservoir simulation output for annual $\mathrm{CO}_{2}$ injection rate during $\mathrm{CO}_{2}$-EGR\&S process with the predicted one by Data-driven proxy model for some of the clusters as well as some of the laterals for all four production and injection patterns/cases. The rest of the results are shown in Appendix B. 


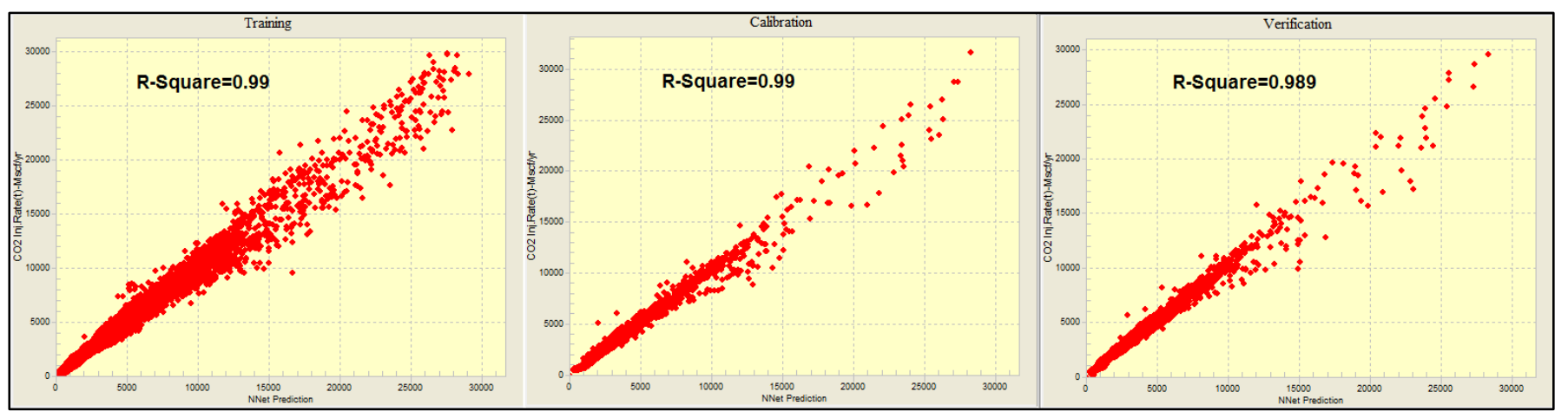

Figure 6-8.NN training, calibration and verification results (From left to right relatively) $-\mathrm{CO}_{2}$ Injection rate (Mscf/year)

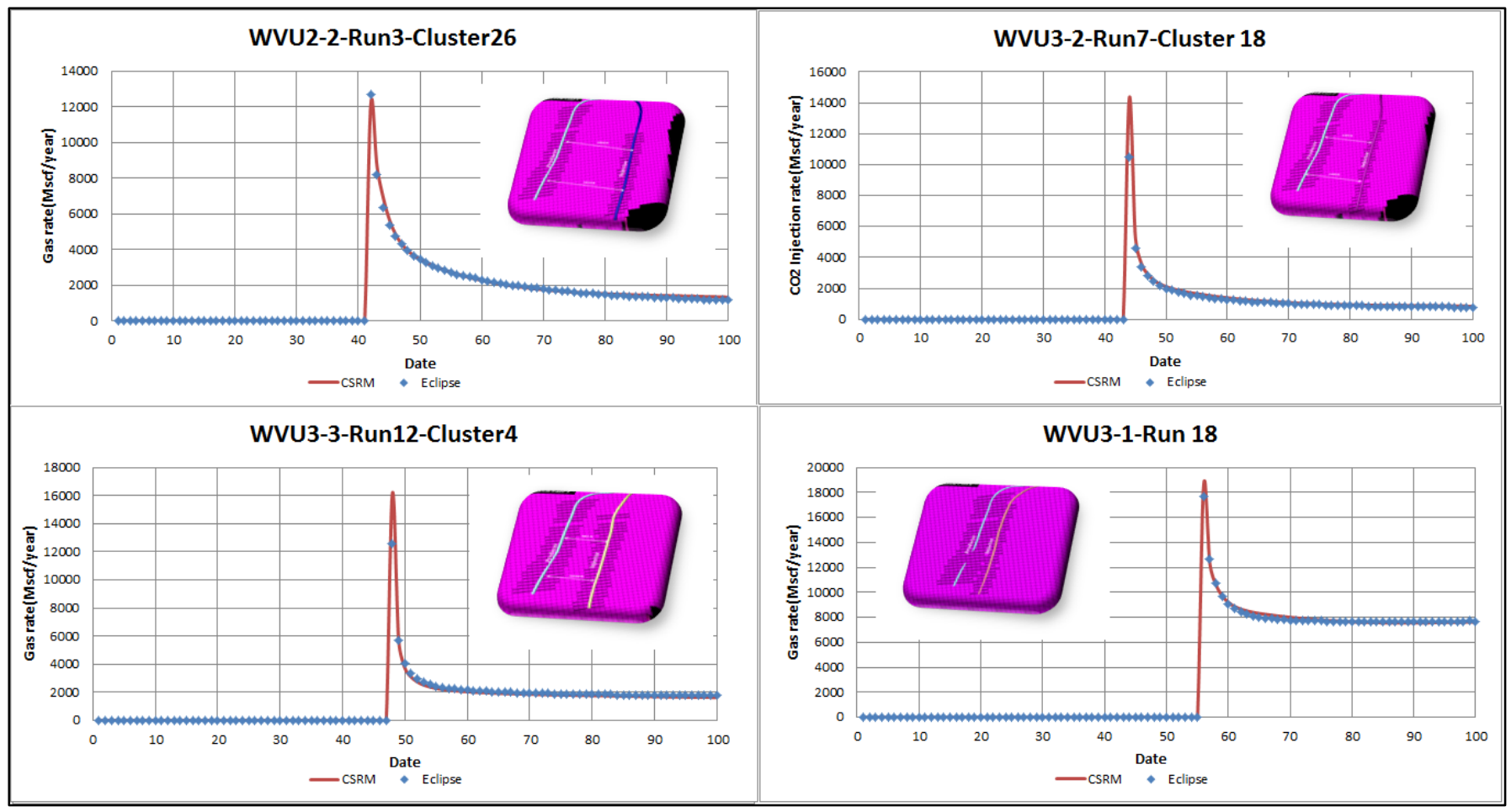

Figure 6-9.Comparison of $\mathrm{CO}_{2}$ injection profile (Mscf/year) from simulator and CO2-EGR\&S Data-driven proxy model for some of the clusters (Cases1, 2, 3 and 4)

In all the plots blue dots represent the $\mathrm{CO}_{2}$ injection rate (Mscf/year) generated by Eclipse and the solid red line is the result of Data-driven $\mathrm{CO}_{2}$-EGR\&S proxy model. The results show that the Data-driven proxy model is predicted the $\mathrm{CO}_{2}$ injection profile at hydraulic fracture cluster, and lateral level for different production/injection patterns with high accuracy. 


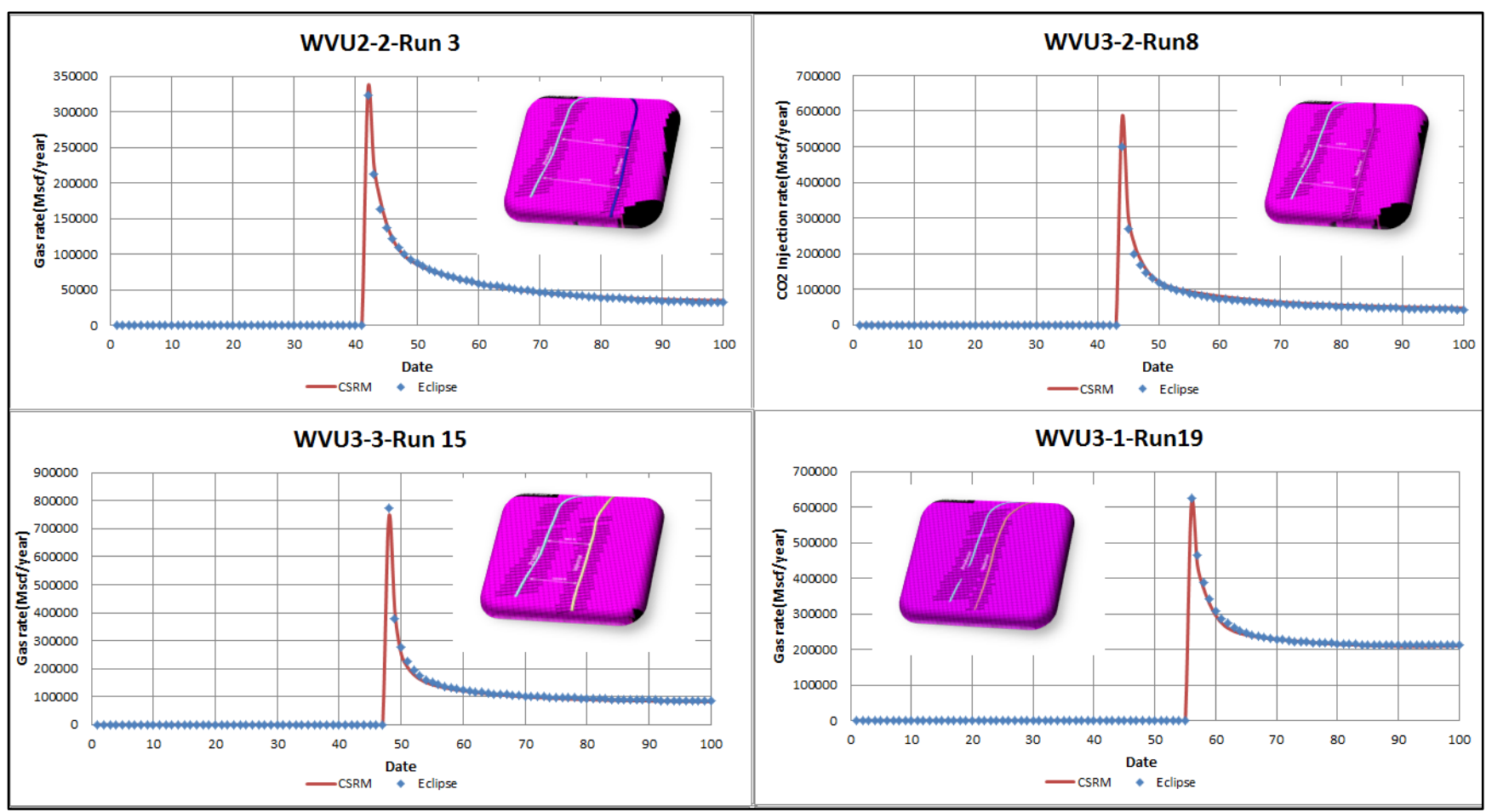

Figure 6-10.Comparison of $\mathrm{CO}_{2}$ injection profile (Mscf/year) from simulator and CO2-EGR\&S Data-driven proxy model for some of the laterals (Cases1, 2, 3 and 4)

\subsection{3 $\mathrm{CO}_{2}$ Breakthrough Time and Production Profile Predication}

The last Data-driven $\mathrm{CO}_{2}$-EGR\&S proxy model is developed to re-generate the $\mathrm{CO}_{2}$ production profile (Mscf/year) from the offset producing well. $\mathrm{CO}_{2}$ breakthrough (BT) occurrence is depending on reservoir characteristics, hydraulic fracture characteristics, sorption features and bottomhole injection pressure. Therefore, the BT time is different in simulation runs.

Since the BT time is one of the inputs to the neural network for the $\mathrm{CO}_{2}$ production profile generation, therefore before starting to build the Data-driven $\mathrm{CO}_{2}$-EGR\&S proxy model for $\mathrm{CO}_{2}$ production rate prediction, developing a proxy model to predict $\mathrm{CO}_{2}$ breakthrough time is a requirement to guarantee the robustness of the final Data-driven EGR\&S proxy model for $\mathrm{CO}_{2}$ production.

Table 6-4 summarizes the list of optimum inputs (42 inputs) that are used for development of a proxy model for $\mathrm{CO}_{2}$ breakthrough time prediction.

The descriptive spatio-temporal database with 1160 pairs of input-output is used to train a multilayer feed-forward back-propagation neural network to predict $\mathrm{CO}_{2}$ breakthrough time. The data set is 
partitioned with $80 \%$ training, and $20 \%$ for calibration and verification. The neural network has one hidden layer with 36 hidden neurons.

Table 6-4.List of optimum number of input for developing Data-driven CO2-EGR\&S proxy model

(CO2Breakthrough time prediction (Day))

\begin{tabular}{|c|c|c|}
\hline \multicolumn{3}{|c|}{ CO2 Breakthrough Time NN- Input Parameters } \\
\hline \multirow{28}{*}{ Static Inputs } & \multirow{20}{*}{$\begin{array}{c}\text { Cluster/Reservoir } \\
\text { Characteristics }\end{array}$} & Location $(X, Y)$ \\
\hline & & Min. Distance to Offset Injector-Producer(Fracture edge) \\
\hline & & Cluster Type \\
\hline & & T1-Km(I)-Marcellus \\
\hline & & T2(-iBound)-Km(I)-Marcellus \\
\hline & & T2(+iBound)-Km(I)-Marcellus \\
\hline & & T3(-jBound)-Km(I)-Marcellus \\
\hline & & T3(-jBound)-Km(I)-Marcellus \\
\hline & & T1-Porosity-Marcellus \\
\hline & & T2(-iBound)-Porosity-Marcellus \\
\hline & & T2(+iBound)-Porosity-Marcellus \\
\hline & & T3(-jBound)-Porosity-Marcellus \\
\hline & & T3(-jBound)-Porosity-Marcellus \\
\hline & & T1-Net Pay-Marcellus \\
\hline & & T2(-iBound)-Net Pay-Marcellus \\
\hline & & T2(+iBound)-Net Pay-Marcellus \\
\hline & & T3(-jBound)-Net Pay-Marcellus \\
\hline & & T3(-jBound)-Net Pay-Marcellus \\
\hline & & $\mathrm{CH} 4 / \mathrm{CO} 2$-Langmuire Pressure \\
\hline & & $\mathrm{CH} 4 / \mathrm{CO} 2$-Langmuire Volume \\
\hline & \multirow{2}{*}{ HF Properties } & Fracture Conductivity in $\mathrm{I}, \mathrm{K}$ \\
\hline & & Hydraulic Fracture half length \\
\hline & \multirow{6}{*}{$\begin{array}{c}\text { Natural Fracture } \\
\text { Properties }\end{array}$} & T1-Kf(I,k)-Marcellus \\
\hline & & T2(-iBound)-Kf(I,k)-Marcellus \\
\hline & & T2(+iBound)-Kf(I,k)-Marcellus \\
\hline & & T3(-jBound)-Kf(I,k)-Marcellus \\
\hline & & T3(-jBound)-Kf(I,k)-Marcellus \\
\hline & & Porosity \\
\hline \multirow{3}{*}{ Dynamic Inputs } & \multicolumn{2}{|r|}{ Time } \\
\hline & \multicolumn{2}{|r|}{ Flowing Bottomhole Pressure } \\
\hline & \multicolumn{2}{|r|}{ Bottomhole Injection Pressure } \\
\hline \multirow{3}{*}{ Additional Inputs } & \multicolumn{2}{|r|}{ Pattern ID } \\
\hline & \multicolumn{2}{|r|}{ Injection start up ID } \\
\hline & \multicolumn{2}{|r|}{ HF-Overlaped_Index } \\
\hline & & 42 Inputs \\
\hline
\end{tabular}


Figure 6-11 illustrates the cross plots for predicted and simulated values of breakthrough time (day) for training, calibration and verification steps (from left to right).In these plots, $x$-axis corresponds to the neural network predicted BT time and the y-axis shows the simulated BT time by Eclipse. The calculated $\mathrm{R}^{2}$ for training, calibration and verification results are around 0.99 .

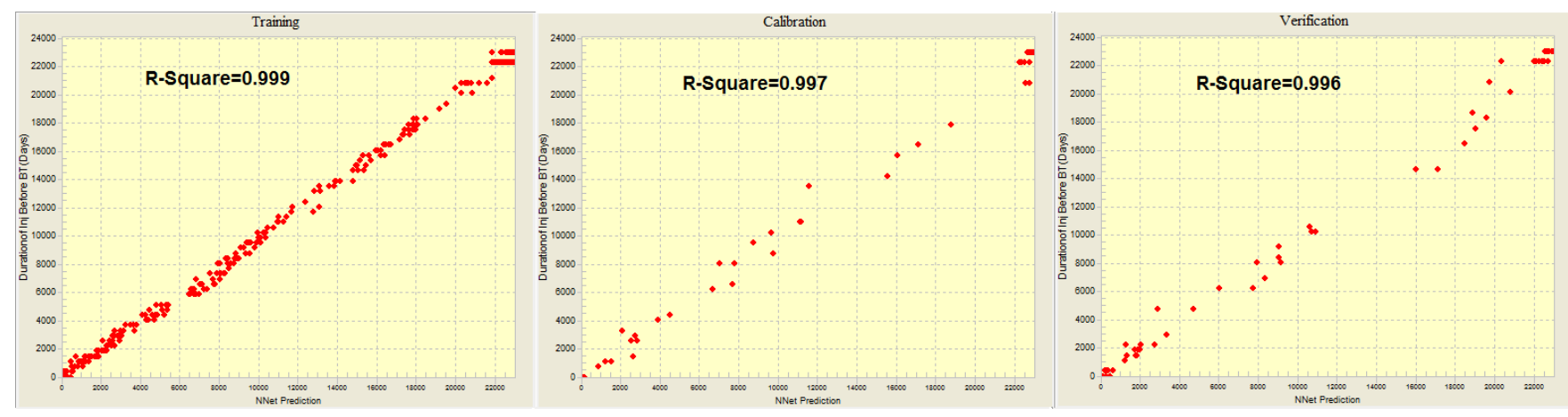

Figure 6-11.NN training, calibration and verification results (From left to right relatively) $-\mathrm{CO}_{2} \mathrm{BT}$ time predication (day)

The next step after the successful development of a proxy model for $\mathrm{CO}_{2}$ breakthrough time prediction is to develop the last Data-driven $\mathrm{CO}_{2}$-EGR\&S proxy model to predict the $\mathrm{CO}_{2}$ production rate (Mscf/year). The same procedure for building the first and second proxies $\left(\mathrm{CH}_{4}\right.$ production and $\mathrm{CO}_{2}$ injection date prediction) is followed here as well.

Table 6-5 summarizes the list of optimum inputs (50 inputs) that are used for development of the Datadriven $\mathrm{CO}_{2}$-EGR\&S proxy model to generate $\mathrm{CO}_{2}$ production profile (Mscf/year).

In order to identify the key parameters that affect the $\mathrm{CO}_{2}$ breakthrough the key performance analysis (KPI) is performed.

According to this analysis, injector/producer pattern, relative location of clusters and $\mathrm{CO}_{2}$ breakthrough time indicator, that was calculated using the $\mathrm{CO}_{2}$ Data-drive proxy for $\mathrm{CO}_{2}$ breakthrough time prediction, and also bottom-hole injection pressure, are essential parameters that control the amount of $\mathrm{CO}_{2}$ production from the offset production well.

The other parameters such as Langmuir isotherms for both $\mathrm{CO}_{2}$ and $\mathrm{CH}_{4}$ and natural fracture permeability and reserve related parameters such as matrix and fracture porosity and pay thickness are the other important parameters that have an impact on the amount of $\mathrm{CO}_{2}$ breakthrough.

As a results designed parameters (i.e. well trajectory, hydraulic fracture placement and operational constraint) should be optimized before stating $\mathrm{CO}_{2}$-Enhamced gas recovery process. 


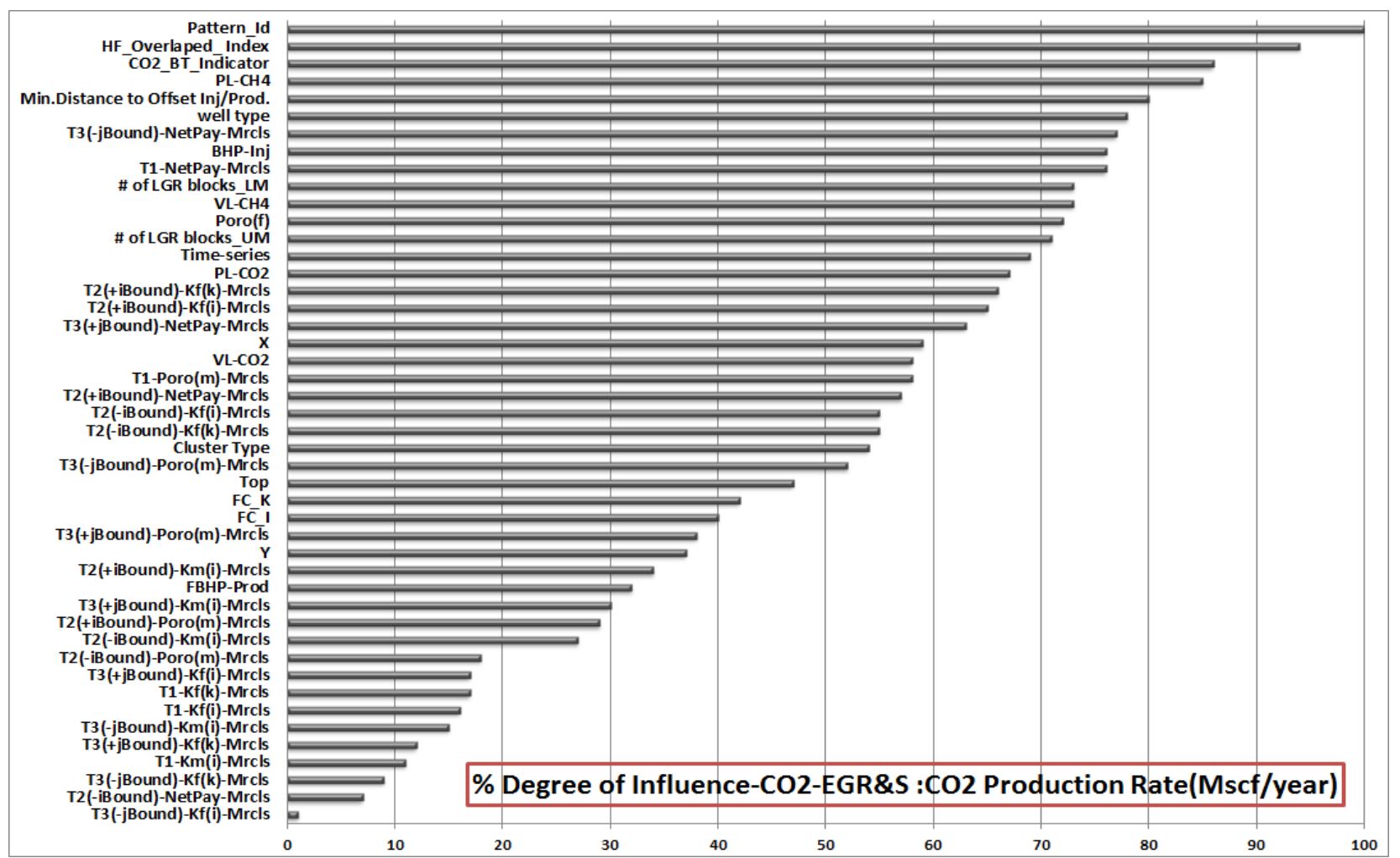

Figure 6-12 Key performance indicators results based on the database for CO2-EGR\&S-CO 2 production rate (Mscf/year)

The representative database with 116, 000 pairs of input-output are used to train a multilayer feed-forward back-propagation neural network. The data is partitioned with $80 \%$ training, and $20 \%$ for calibration and verification. The designed neural network has one hidden layers with 52 hidden neurons.

The cross plots for predicted and simulated values of $\mathrm{CO}_{2}$ production rate (Mscf/year) for training, calibration and verification steps (from left to right) are shown in Figure 6-13. In these plots, x-axis corresponds to the neural network predicted $\mathrm{CO}_{2}$ production rate (Mscf/year) and the y-axis shows the simulated $\mathrm{CO}_{2}$ production rate by the reservoir simulator (Eclipse).

The Data-driven $\mathrm{CO}_{2}$-EGR\&S proxy model is successfully developed to predict the $\mathrm{CO}_{2}$ production rate (Mscf/year) with an $\mathrm{R}^{2}$ of more than 0.99 for training, calibration and verification.

Figure 6-14 and Figure 6-15 show some examples of the comparison of reservoir simulation output (Blue dots) for $\mathrm{CO}_{2}$ production rate (Mscf/year) during $\mathrm{CO}_{2}$-EGR\&S process with the predicted one by Datadriven proxy model (Red solid line) for some of the clusters as well as some of the laterals for all four production and injection patterns/cases. The plots clearly show that the developed Data-driven $\mathrm{CO}_{2}$ EGR\&S proxy model is capable of re-generating the $\mathrm{CO}_{2}$ production rate with acceptable accuracy. The rest of the results for all the runs are shown in Appendix B. 
Table 6-5.List of optimum number of input for developing Data-driven CO2-EGR\&S proxy model

(Annual CO2 Production Rate)

\begin{tabular}{|c|c|c|}
\hline \multicolumn{3}{|c|}{ CO2 Production NN- Input Parameters } \\
\hline \multirow{30}{*}{ Static Inputs } & \multirow{22}{*}{$\begin{array}{c}\text { Cluster/Reservoir } \\
\text { Characteristics }\end{array}$} & Location $(X, Y)$ \\
\hline & & Min. Distance to Offset Injector-Producer(Fracture edge) \\
\hline & & Top \\
\hline & & Cluster Type \\
\hline & & T1-Km(I)-Marcellus \\
\hline & & T2(-iBound)-Km(I)-Marcellus \\
\hline & & $\mathrm{T} 2$ (+iBound)-Km(I)-Marcellus \\
\hline & & $\mathrm{T} 3(-\mathrm{jBound})-\mathrm{Km}(\mathrm{I})$-Marcellus \\
\hline & & T3(-jBound)-Km(I)-Marcellus \\
\hline & & T1-Porosity-Marcellus \\
\hline & & T2(-iBound)-Porosity-Marcellus \\
\hline & & T2(+iBound)-Porosity-Marcellus \\
\hline & & T3(-jBound)-Porosity-Marcellus \\
\hline & & T3(-jBound)-Porosity-Marcellus \\
\hline & & T1-Net Pay-Marcellus \\
\hline & & T2(-iBound)-Net Pay-Marcellus \\
\hline & & T2(+iBound)-Net Pay-Marcellus \\
\hline & & T3(-jBound)-Net Pay-Marcellus \\
\hline & & T3(-jBound)-Net Pay-Marcellus \\
\hline & & $\mathrm{CH} 4 / \mathrm{CO} 2$-Langmuire Pressure \\
\hline & & $\mathrm{CH} 4 / \mathrm{CO}$-Langmuire Volume \\
\hline & & CO2 Diffusion coefficient \\
\hline & \multirow{2}{*}{ HF Properties } & Fracture Conductivity in I,K \\
\hline & & Hydraulic Fracture half length \\
\hline & \multirow{6}{*}{$\begin{array}{c}\text { Natural Fracture } \\
\text { Properties }\end{array}$} & T1-Kf(I,k)-Marcellus \\
\hline & & T2(-iBound)-Kf(I,k)-Marcellus \\
\hline & & $\mathrm{T} 2$ (+iBound)-Kf(I,k)-Marcellus \\
\hline & & T3(-jBound)-Kf(I,k)-Marcellus \\
\hline & & T3(-jBound)-Kf(I,k)-Marcellus \\
\hline & & Porosity \\
\hline \multirow{3}{*}{ Dynamic Inputs } & \multicolumn{2}{|r|}{ Time } \\
\hline & \multicolumn{2}{|r|}{ Flowing Bottomhole Pressure } \\
\hline & \multicolumn{2}{|r|}{ Bottomhole Injection Pressure } \\
\hline \multirow{3}{*}{ Additional Inputs } & \multicolumn{2}{|r|}{ Pattern ID } \\
\hline & \multicolumn{2}{|r|}{ HF-Overlaped_Index } \\
\hline & \multicolumn{2}{|r|}{ CO2-BT time indicator } \\
\hline \multicolumn{3}{|r|}{48 Inputs } \\
\hline
\end{tabular}
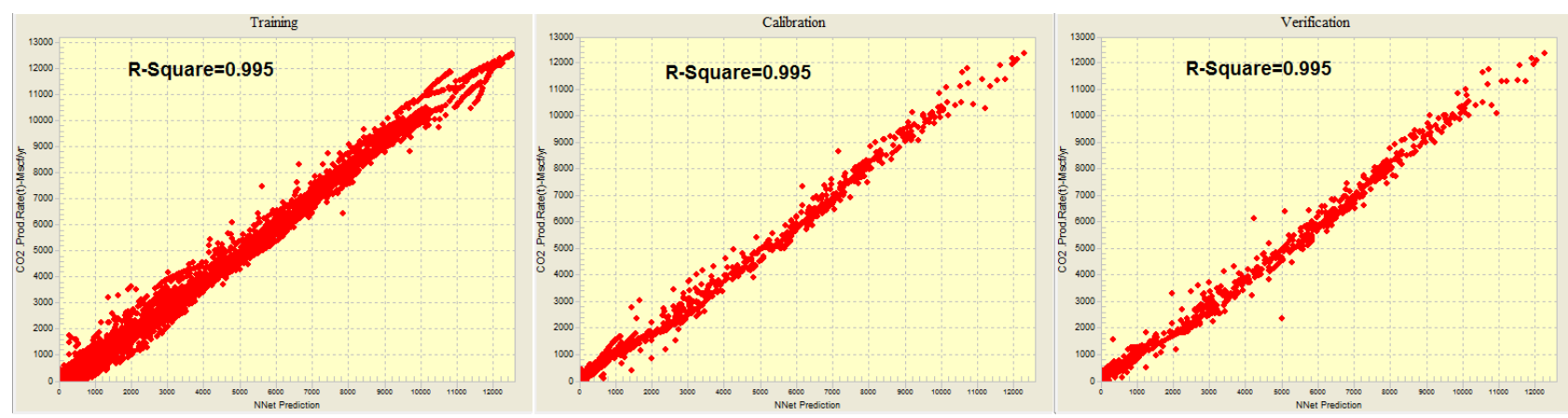

Figure 6-13.NN training, calibration and verification results (From left to right relatively) - CO2 Production predication (Mscf/year) 


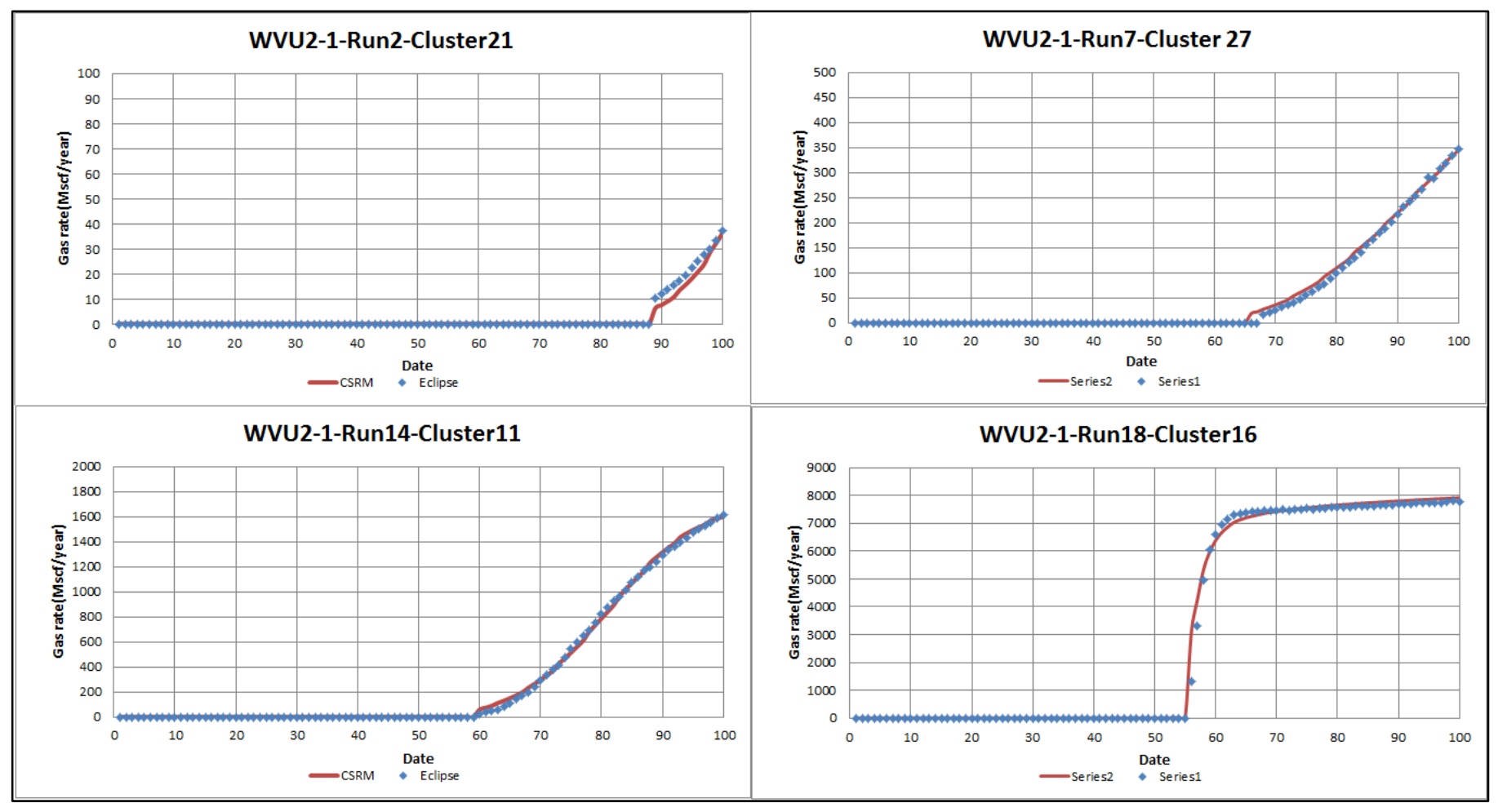

Figure 6-14.Comparison of $\mathrm{CO}_{2}$ production profile (Mscf/year) from simulator and CO2-EGR\&S Data-driven proxy model for some of the hydraulic fracture clusters (Cases1, 2, 3 and 4)

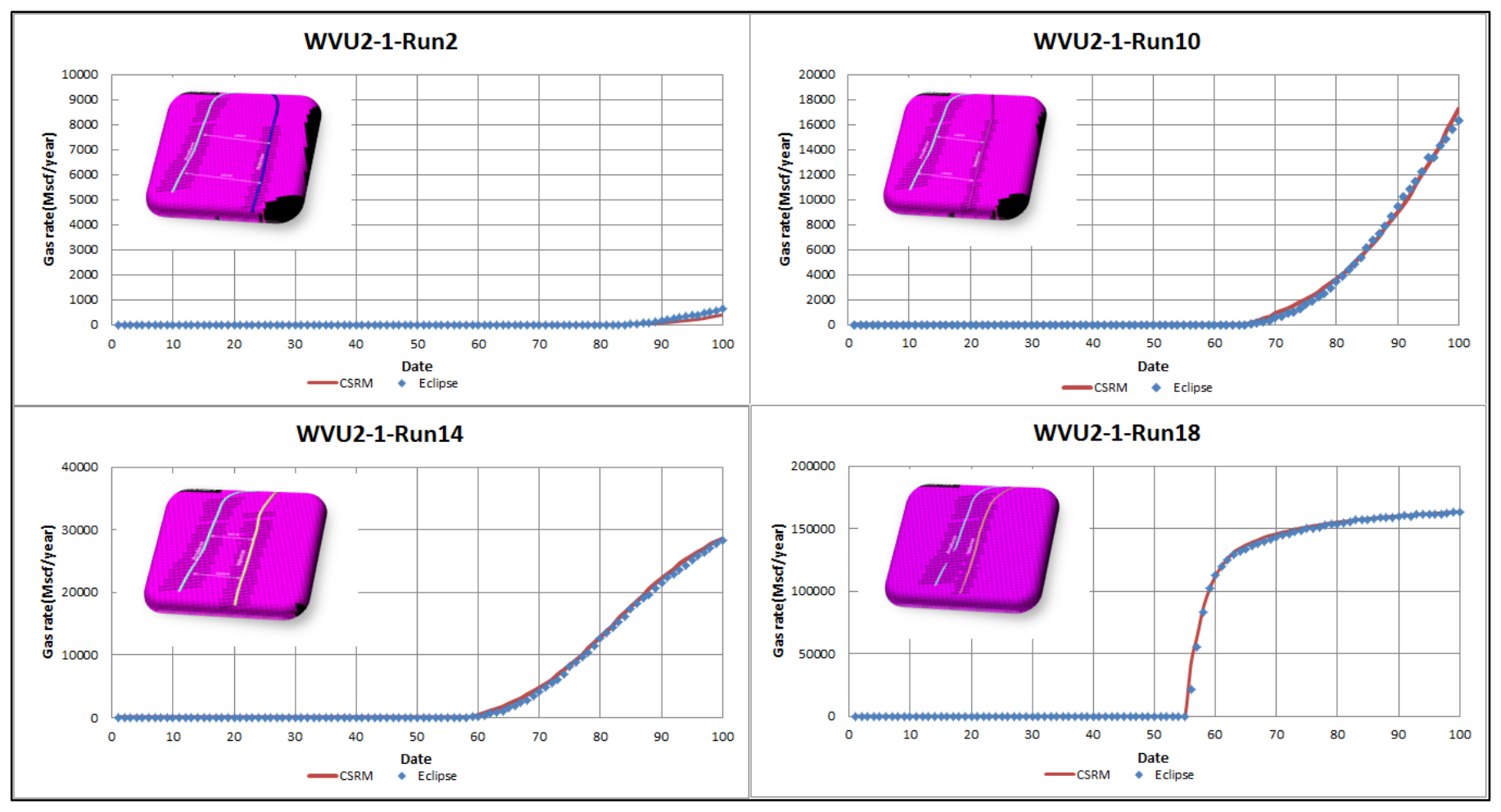

Figure 6-15.Comparison of $\mathrm{CO}_{2}$ production profile (Mscf/year) from simulator and CO2-EGR\&S Data-driven proxy model for some of the laterals (Cases1, 2, 3 and 4) 


\subsection{Data-driven $\mathrm{CO}_{2}$-EGR\&S Proxy Model Validation by Blind Cases}

Validating/testing the developed Data-driven proxy model is the final step of the process to examine the capability of the model in predictive mode. In order to do that, one of the cases (Case2) including five simulation runs is completely removed from the training and initial validation process. The same input parameters that were used for developing the four Data-driven $\mathrm{CO}_{2}$-EGR proxy models $\left(\mathrm{CH}_{4}\right.$ Production, $\mathrm{CO}_{2}$ Injection rates, $\mathrm{CO}_{2}$ Breakthrough time and $\mathrm{CO}_{2}$ production rate prediction) are used for developing new proxy model based on 15 simulation runs.

Upon completion of the training and validation of the new proxy models, the inputs (i.e. Static properties and operational constraints) for the five simulation runs (with 58 clusters of hydraulic fracture per run) are introduced to the proxy models. The corresponding outputs including $\mathrm{CH}_{4}$ Production, $\mathrm{CO}_{2}$ Injection rates (Mscf/year), $\mathrm{CO}_{2}$ Breakthrough time (day) and $\mathrm{CO}_{2}$ production rate (Mscf/year) are generated and some of the results are illustrated in Figure 6-16 $\left(\mathrm{CH}_{4}\right.$ production rate), Figure 6-17 $\left(\mathrm{CO}_{2}\right.$ injection rate) and Figure 6-18 $\left(\mathrm{CO}_{2}\right.$ production rate). In all the plots, the blue dots are the simulation outputs and the red solid lines are the proxy model results.

As can be seen from Figure 6-16, the Data-driven $\mathrm{CO}_{2}$-EGR\&S proxy model is predicted the methane production rates for both production and the prospective injector with acceptable accuracy. Since hydraulic fracture length and number of clusters for WVU2-1 does not change for all 15 runs, therefore the neural network could capture the production behavior of this well better than WVU3-2.

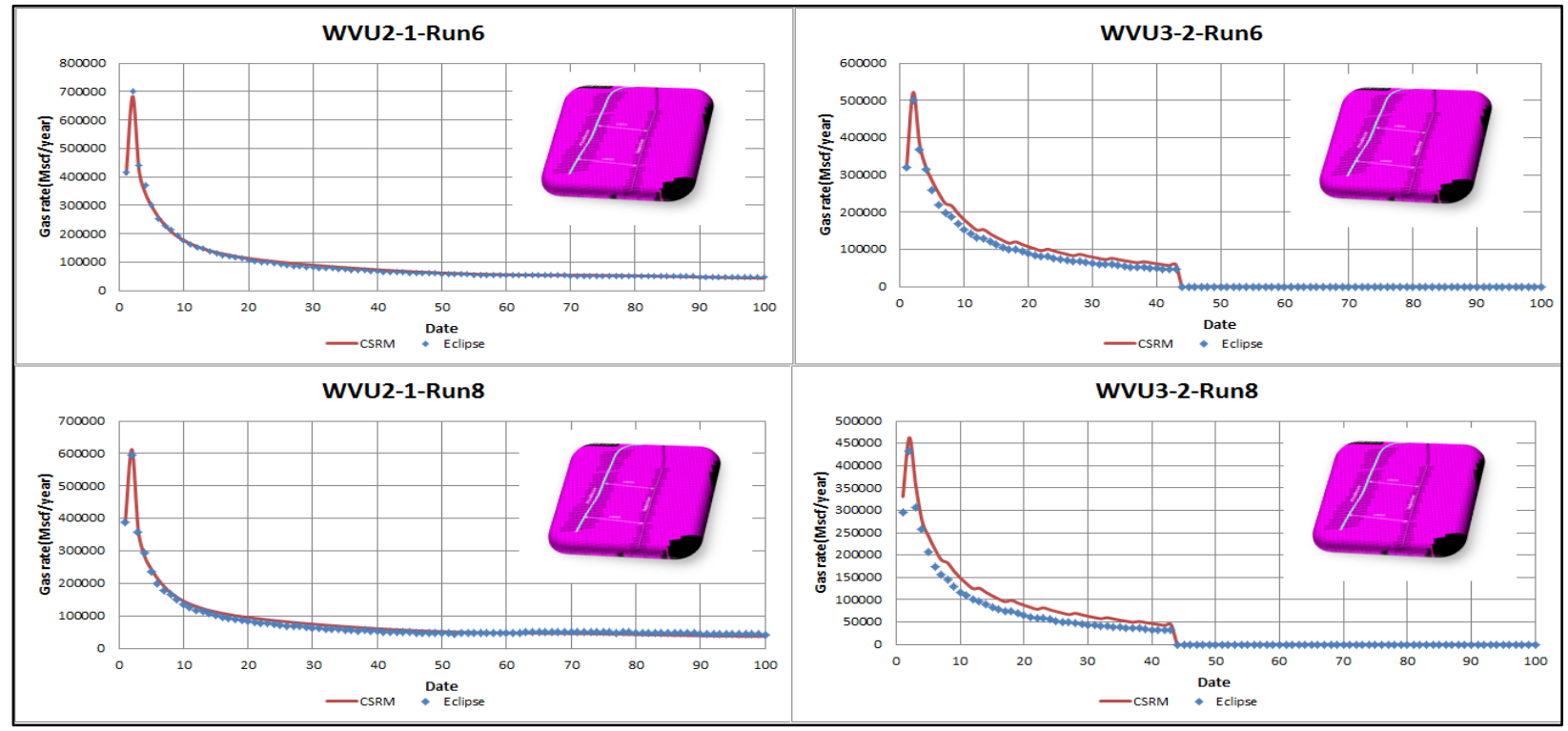

Figure 6-16.Comparison of $\mathrm{CH}_{4}$ production profile (Mscf/year) from simulator and CO2-EGR\&S Data-driven proxy model for some of the laterals -Blind Case 


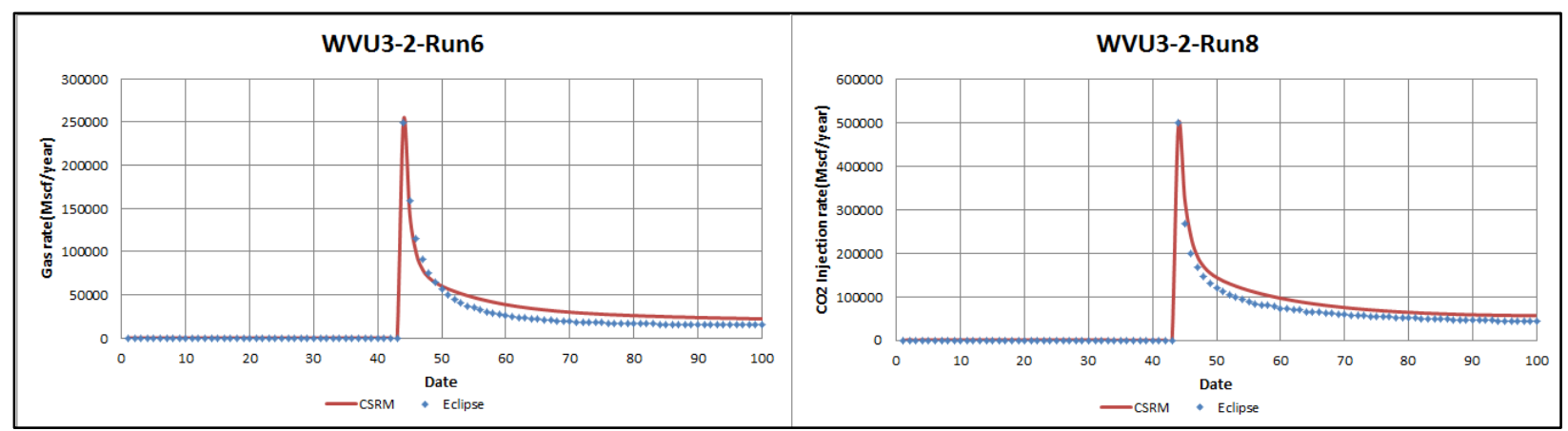

Figure 6-17.Comparison of $\mathrm{CO}_{2}$ injection profile (Mscf/year) from simulator and CO2-EGR\&S Data-driven proxy model for some of the laterals -Blind Case

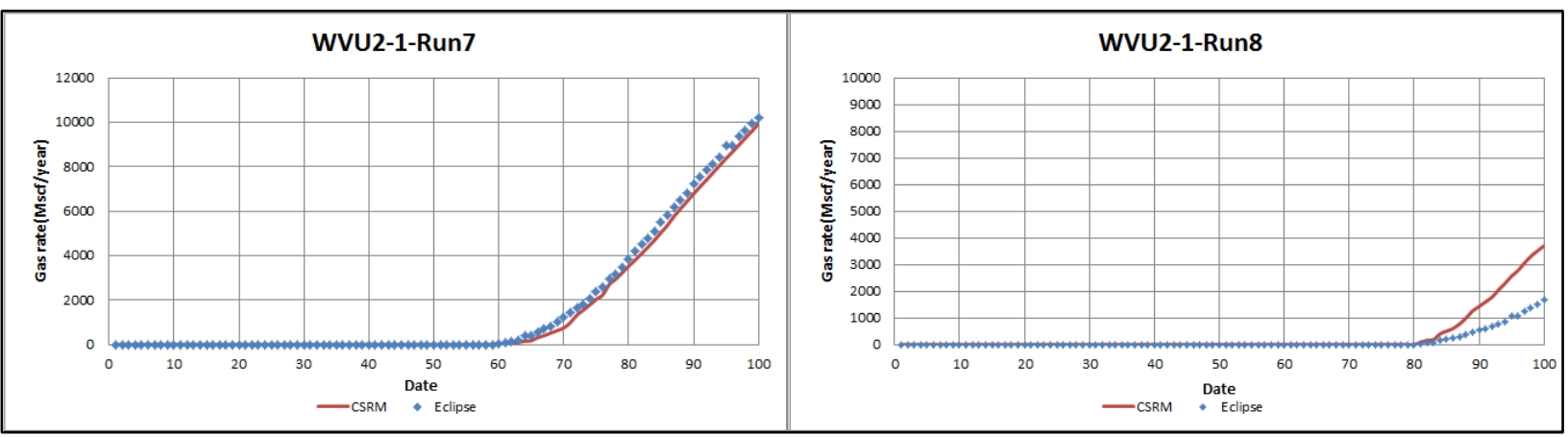

Figure 6-18.Comparison of $\mathrm{CO}_{2}$ production profile (Mscf/year) from simulator and CO2-EGR\&S Data-driven proxy model for some of the laterals -Blind Case

Figure 6-17 shows the comparison of the $\mathrm{CO}_{2}$ injection profile generated using Eclipse and the proxy model predicted one for some of the runs, which were completely blind to the neural network.

According to the Figure 6-18, the $\mathrm{CO}_{2}$ production profile generated by Data-driven proxy model was not able to mimic the simulation behavior with very high accuracy. The reason for that is, while designing the simulation runs for $\mathrm{CO}_{2}$-EGR\&S process the main objective of minimizing $\mathrm{CO}_{2}$ breakthrough was considered. Therefore, in most of the cases, $\mathrm{CO}_{2}$ breakthrough is not an issue and even if it happens, the amount of daily $\mathrm{CO}_{2}$ breakthrough is within the economic limit except for the Case 4, which the hydraulic fractures are overlapped. As a result, when five simulation runs were removed from the spatio-temporal database for further validation the number of breakthrough occurrence was decreased in the database therefore the $\mathrm{NN}$ does not have enough information to learn from.

The second way for validating/testing the capability of the developed Data-driven proxy model is to design a completely new horizontal well with unique reservoir and hydraulic fracture characteristics for each cluster and with new operational constraints, which are completely different (but in the uncertainty range) from the initially designed 20 simulation runs for training the neural network. Therefore, a new synthetic well (WVU-2013) is drilled and completed with 25 clusters of hydraulic fractures. 


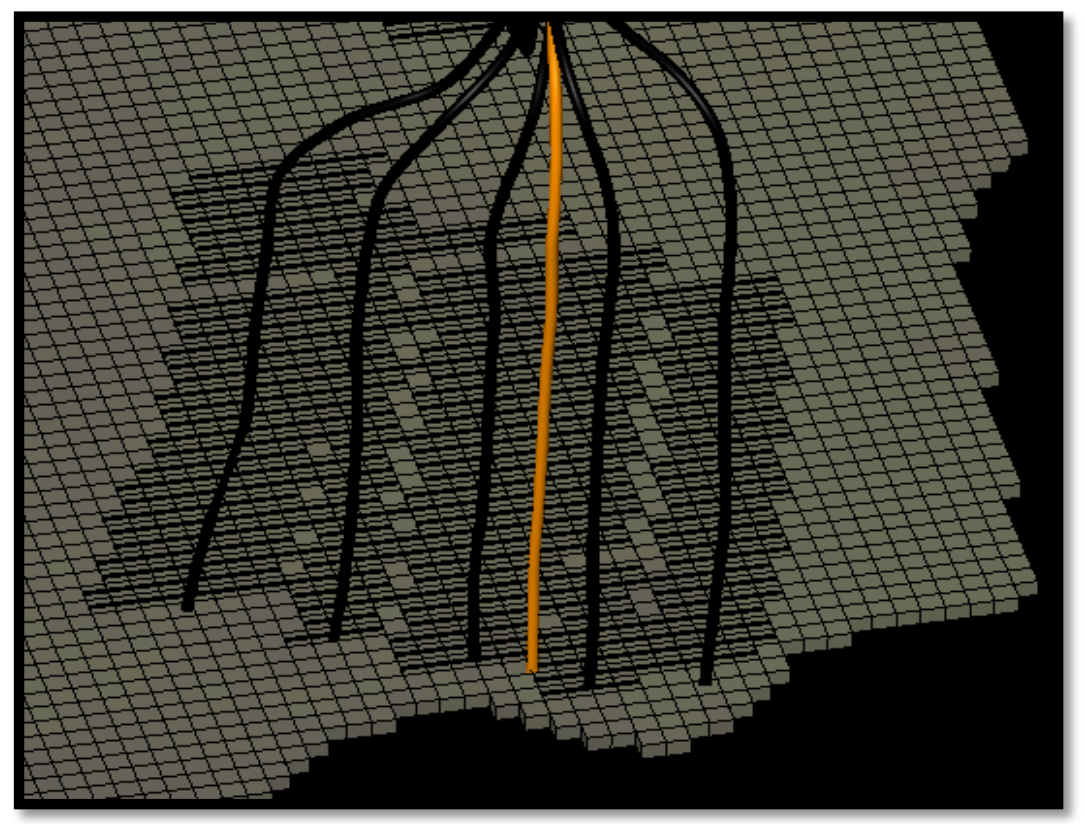

Figure 6-19. Schematic of new synthetic well for validation of the proxy models

Figure 6-19 shows the horizontal well trajectory with orange color and its relative position to the other offset laterals in the WVU pad. The simulation outputs are compared with Data-driven proxy model results and are shown in the Figure 6-20. According to this figure, the top figures are the comparison

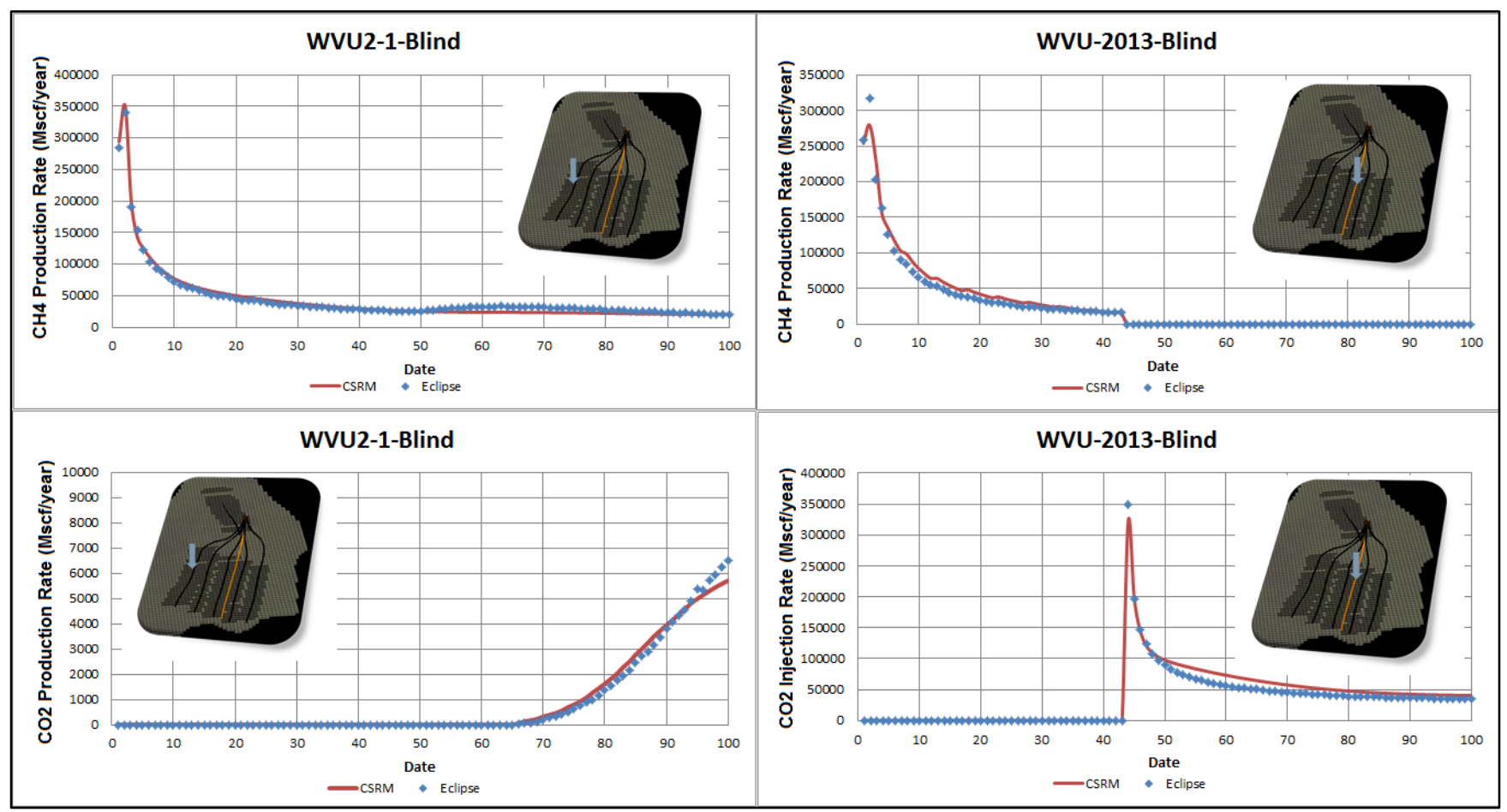

Figure 6-20. Comparison of $\mathrm{CH}_{4}, \mathrm{CO}_{2}$ production profiles (Mscf/year) and $\mathrm{CO}_{2}$ Injection rate (Mscf/year) from simulator and CO2-EGR\&S Data-driven proxy model for some of the laterals -Blind Case 
Table 6-6.Comparison of some of the discrete parameters' values in the training dataset and the blind simulation runs

Input parameters

Training set range

Blind run value

\begin{tabular}{|c|c|c|}
\hline Fracture Porosity (\%) & {$[0.5,1.25,2,2.75,3.5]$} & 2.3 \\
\hline Sigma & {$[0.005,0.024,0.042,0.062,0.081]$} & 0.035 \\
\hline VL_CH $\mathrm{CH}_{4}(\mathrm{Mscf} /$ ton $)$ & {$[0.055,0.064,0.073,0.082,0.091]$} & 0.06 \\
\hline $\mathrm{PL}_{2} \mathrm{CH}_{4}(\mathrm{psi})$ & {$[600,675,750,825,900]$} & 700 \\
\hline $\mathrm{VL} \_\mathrm{CO}_{2}(\mathrm{Mscf} /$ ton $)$ & {$[0.07,0.095,0.12,0.145,0.17]$} & 0.1 \\
\hline $\mathrm{PL}_{-} \mathrm{CO}_{2}$ (psi) & {$[400,450,500,550,600]$} & 475 \\
\hline Diffusion coefficient_CH$\left(\mathrm{Ct}_{4} / \mathrm{day}\right)$ & {$[0.2,1.15,2.1,3.05,4]$} & 2.7 \\
\hline P_Injection (psi) & {$[1680,2100,2520,2940,3360]$} & 2250 \\
\hline
\end{tabular}

of simulation and proxy model results for the methane production for the producing and the prospective injector wells. The bottom left plot shown the $\mathrm{CO}_{2}$ production and the bottom right figure shows the $\mathrm{CO}_{2}$ injection profile comparisons.

Table 6-6 shows the comparison of the range of values for some of the parameters in the training dataset with the ones that are used to design the blind simulation runs.

In general, the developed Data-driven $\mathrm{CO}_{2}$-EGR\&S proxy models can be effectively used as a tool for uncertainty quantification since thousands of proxy models runs can be made in a few seconds to fulfill the requirement of the Monte Carlo simulation for uncertainty analysis.

\subsection{Summary and Conclusions}

In this chapter, Data drive $\mathrm{CO}_{2}$-EGR\&S proxy models for shale formation are developed based on pattern recognition capabilities of Artificial Intelligence is introduced, developed and validated by completely blind simulation runs to reproduce the injection and production profiles for the $\mathrm{CO}_{2}$-Enhanced gas recovery and storage process.

This technique provides assistance to perform detailed uncertainty and optimization analysis very fast instead of directly using the numerical simulator that the model set up and its implementation, are laborious and computationally expensive.

Additionally, key performance analysis is performed by using fuzzy pattern recognition technique and the most influential parameters that control $\mathrm{CH}_{4}$ production rate, $\mathrm{CO}_{2}$ injection rate, $\mathrm{CO}_{2}$ breakthrough time and $\mathrm{CO}_{2}$ production rate are identified to be considered before designing any $\mathrm{CO}_{2}$ injection process for the purpose of enhanced gas recovery and storage. 


\section{CHAPTER 7 -CONCLUSIONS AND RECOMMENDATIONS}

\subsection{Concluding Remarks}

The major conclusions of this work can be summarized as follows:

1- An integrated workflow, which demonstrates a quantitative platform for shale gas production modeling and simulation through capturing the essential characteristics of the shale gas reservoir is proposed. In this workflow, dual porosity, compositional simulation with explicit representation of hydraulic fracture is used to perform history matching and production forecast. A representative pad in terms of reservoir and hydraulic fracture characteristics in the large Marcellus shale study area is selected to perform the simulation. History matching process is successfully completed for all the laterals (six horizontal laterals in WVU Pad) and the production is forecasted for 90 years.

2- Almost all the numerical simulation studies performed on shale formations so far, are single well models because of long and laborious model setup and computationally expensive implementation of it, and also the interferences between the hydraulic fractures in a Pad with multilateral makes the history matching job very difficult. Therefore, there is a need to develop a technique to make this process easier and performing uncertainty analysis more efficient.

3- A Next-generation shale proxy model based on pattern recognition capabilities of Artificial Intelligence is introduced to assist in shale gas modeling and simulation in the case of using EHF technique. Data-driven proxy models are developed for three different time resolutions of daily, monthly and annually that are able to generate production profile for each cluster with acceptable accuracy. This technique enables the shale modelers to use all functionality of the developed simulation model and predict shale gas production behavior at each cluster faster with high accuracy compared with the numerical simulator. Moreover, it can be used as an assisted historymatching tool and also for uncertainty quantification and designing new wells with different clusters of hydraulic fracture. Because the modeling is performed at HF cluster level, the Datadrive proxy model is robust enough to predict the new well production profile with any definite number of hydraulic fractures and any assigned properties in uncertainty domain.

4- A comprehensive simulation studies are performed to assess the potential of shale gas reservoir for $\mathrm{CO}_{2}$ storage and to investigate the $\mathrm{CO}_{2}$ enhanced gas recovery process. For economically viable enhanced gas recovery and storage process, a large volume of accessible gas to the producing lateral, which is going to be converted to an injector, should be produced before the 
injection starts up. Therefore, before starting the injection process, all the wells in each simulation case are put on production for 100 years that is called base injection case. When one of the production wells, which is going to be converted to an injection well, produced $75 \%$ of its accessible gas, the $\mathrm{CO}_{2}$ injection starts and continues until the end of the $\mathrm{t}=100$ years. This usually happens between 45 to 55 years after the start of production.

5- It is impractical to inject $\mathrm{CO}_{2}$ into the laterals in a pad. This is due to the fact that communication between hydraulic fractures in the so-called SRV regions contributes to early breakthrough. Therefore, the pairs of injector/producer are selected with appropriate spacing.

6- Depleted history matched model is used to perform the analysis based on different injection/production patterns and different reservoir characteristic, hydraulic fracture properties and sorption features.

7- By designing different injection scenarios, the best practices to enhance methane recovery, minimize $\mathrm{CO}_{2}$ production while maximizing the amount of stored $\mathrm{CO}_{2}$ in shale formation are proposed. According to this study, the best injection scenario is the one with two producers and one injector in the middle with 900-970 ft. spacing from the offset producing wells.

8- Based on our analysis, the best bottomhole injection pressure varies from 25 to $50 \%$ of lithostatic pressure to maximize $\mathrm{CO}_{2}$ storage while delaying the $\mathrm{CO}_{2}$ breakthrough time.

9- The $\mathrm{CO}_{2}$ plume extension will not go beyond the so-called SRV region.

10- One of the key components of a $\mathrm{CO}_{2}$-EGR\&S process is considering the impact of sorption isotherms, which have significant impact on $\mathrm{CO}_{2}$ injectivity, $\mathrm{CO}_{2}$ breakthrough time and methane recovery.

11- In order to provide assistance to perform detailed uncertainty and optimization analysis for the $\mathrm{CO}_{2}$-Enhanced gas recovery and storage process, Data drive $\mathrm{CO}_{2}$-EGR\&S proxy models for shale reservoir are developed based on pattern recognition capabilities of Artificial Intelligence. The developed proxies are capable of re-generating the numerical simulation results for $\mathrm{CH}_{4}$ production rate, $\mathrm{CO}_{2}$ injection rate, $\mathrm{CO}_{2}$ breakthrough time and $\mathrm{CO}_{2}$ production rate with high accuracy. The proxy models are validated by completely blind runs to reproduce the injection and production profiles that are generated by the blind simulation runs at hydraulic fracture cluster/lateral levels.

12- Additionally, key performance analysis is performed by using fuzzy pattern recognition technique and the most influential parameters that control $\mathrm{CH}_{4}$ production rate, $\mathrm{CO}_{2}$ injection 
rate, $\mathrm{CO}_{2}$ breakthrough time and $\mathrm{CO}_{2}$ production rate are identified to be considered before designing any $\mathrm{CO}_{2}$ injection process for the purpose of enhanced gas recovery and storage.

\subsection{Recommendation for Future Work}

The following items are recommended for future work in this area:

1- Before starting the history matching process, first a Data-driven proxy model can be developed to be used as an assisted history-matching tool in order to facilitate the history matching process.

2- The history matched model can be updated with longer production history data and also the injection scenarios can be validated by the actual data from future pilot tests for $\mathrm{CO}_{2}$-EGR\&S in shale reservoir.

3- Instead of using a single pad for performing the analysis, multiple pads can be used as injector/producer pairs in the case of having access to more powerful computers. 


\section{REFERENCES}

Al-Ahmadi, H.A., Almarzooq, A.M., Wattenbarger, R.A., “Application of Linear Flow Analysis to Shale GasWells e Field Cases" SPE 130370, SPE Unconventional Gas Conference, Pittsburgh, PA, 23-25 February, 2010.

Andrade, J., Civan, F., Devegowda, D., Sigal, R.”Accurate Simulation of Shale Gas Reservoirs" SPE135564. Annual Technical Conference and Exhibition held in Florence, Italy, September 19-22, 2010.

Anderson, D.M., Nobakht, M., Moghadam, S., Mattar, L., “Analysis of Production Data From Fractured Shale Gas Wells", SPE 131787, SPE Unconventional Gas Conference, Pittsburgh, PA, February 23-25, 2010.

Andrade, J., Civan, F., Devegowda, D., Sigal, R. F. "Design and examination of requirements for a rigorous shale-gas reservoir simulator compared to current shale gas simulators" SPE 144401, North American Unconventional Gas Conference and Exhibition, Woodlands, Texas, June 14-16, 2011.

Barber.S,:”AI : Neural Network for beginners (Part 1 of 3)" Code project ,2007

Barlow, H. B. Unsupervised learning. Neural Computation 1, 295-311, 1988.

Barenblatt, G. I., Zeltov, Y. P., and Kochina, I. "Basic concepts in the theory of seepage of homogeneous liquids in fissured rocks" PMM (Journal of Soviet Applied Mathematics and Mechanics), 24(5): 12861303(1960).

Bezdek,J, Pal,S.'Fuzzy Models for Pattern Recognition-Methods That Search for Structures in Data" IEEE Press 1992, p.xi. A Selected Reprint Volume-IEEE Neural Networks Council.

Beskok, A., Karniadakis, G. E. "A model for flows in channels, pipes, and ducts at micro and nano scales” Microscale Thermophys Eng. 3(1), 43-77(1999).

Bello, R.O., Wattenbarger, R.A., "Rate Transient Analysis in Naturally Fractured Shale Gas Reservoirs" SPE 114591, CIPC/SPE Gas Technology Symposium e Joint Conference, Calgary, Alberta, 16-19 June ,2008. 
Blaskovich, F.T., Gain, G.M., and Sonier, F., "A multi-component isothermal system for efficient reservoir simulation, SPE 11480, Middle East Oil Technical Conference, Bahrain, March 14-17, 1983.

Boulis, A.S., Ilk, D., Blasingame, T.A., “A New Series of Rate Decline Relations Based on the Diagnosis of Rate-Time Data” Canadian International Petroleum Conference (CIPC) ,Calgary, Alberta, June 16-18, 2009.

Can, B., Kabir, C.S., "Probabilistic performance forecasting for unconventional reservoirs with stretchedexponential model" SPE 143666. SPE Reservoir Evaluation and Engineering Journal, 41-50, February 2012.

Chakraborty RC:" Fundamentals of Neural Networks: AI course” Lecture 37-38, June 2010 http://www.myreaders.info/html/artificial_intelligence.html

Cheng, Y., Wang, Y., McVay, D.A., Lee, W.J., "Practical application of a probabilistic approach to estimate reserves using production decline data " SPE Economics \& Management Journal, 19-31, April 2010.

Ciezobka, J., “Marcellus Shale Gas Project”, RPSEA, Annual Report, 2012.

Civan, F. "Effective correlation of apparent gas permeability in tight porous media" Transport in Porous Media, 2010. Vol.82:375-384.

Cipolla, C.L. et al: "Modeling Well performance in Shale-Gas Reservoirs," SPE 125532, SPE/WAGE Reservoir Characterization, and Simulation Conference held in Abu Dhabi, UAE, Oct. 19 - 21.2009

Cipolla, C.L., Lolon, E.P., Erdle, J.C., and Rubin, B. "Reservoir Modeling in Shale-Gas Reservoirs" SPE Res. Eval. \& Eng. 13 (4): 638-653 (2010)

Clarkson, C. R., Ertekin, T. "A new model for shale gas matrix flow using the dynamic-slippage concept" AAPG Hedberg Conference, Austin, Texas, December 5-10, 2010

Clarkson C.R., Jensen, J.L and Blasingame T.A.;" Reservoir Engineering for Unconventional Gas Reservoirs: What Do We Have to Consider?" SPE 145080, North American Unconventional Gas Conference and Exhibition, Texas, 14-16 June 2011. 
Darishchev A., Lemouzy P., Rouvroy P., "On Simulation of Flow in Tight and Shale Gas Reservoirs" SPE 163990, Middle East Unconventional Gas Conference and Exhibition held in Muscat, Oman, 28-30 January 2013.

Dean, R.H. and Lo, L., "Development of a naturally fractured reservoir simulator and examples of its use" SPE 14110, International Meeting on Petroleum Engineering, Beijing, China, March 17-20,1986.

deSwaan-O, A. "Analytic solutions for determining naturally fractured reservoir properties by well testing” SPE Journal (June 1976) 117-122; Trans., AIME 261.

Ennis-King J. and Paterson L., "Engineering aspects of geological sequestration of carbon dioxide" in Proceedings of the SPE Asia Pacific Oil and Gas Conference and Exhibition, pp. 134-146, Melbourne, Australia, October 2002.

Ertekin, T., King, G. R., Schwerer, F. C.’Dynamic gas slippage: a unique dual-mechanism approach to the flow of gas in tight formations" SPE Formation Evaluation, 1(1): 43-52. SPE 12045-PA doi: 10.2118/12045-PA (1986)

Essendelft Dirk Van, Taron J., Fitzgerald M., Abdel-Hafez A., Sakti A., Achimugu O., Faoro I.:" $\mathrm{CO}_{2}$ Sequestration through Deep Saline Injection and Photosynthetic Biological Fixation: System Design for Two Plausible $\mathrm{CO}_{2}$ Sequestration Strategies" Final report -Department of Energy and Geo-Environmental Engineering, The Pennsylvania State University,2006

Fathi E, Akkutlu Y:" Nonlinear Sorption Kinetics and Nonlinear Sorption Kinetics and Surface Diffusion Effects on Gas Transport in Low-permeability Formations" SPE 124478, Annual Technical Conference and Exhibition held in New Orleans, Louisiana, USA, 4-7 October 2009.

Fausett, L. "Fundamentals of Neural Networks, Architectures, Algorithms, and Applications", Prentice Hall, Englewood Cliffs, NJ, 1994.

FracPro-Carbo Ceramics, http://www.carboceramics.com/fracpropt-software/

Goodvin, N, Powell, M: "Simulation and Uncertainty: Lessons From Other Industries" THEWAYAHEAD Journal, Vol.8, No.3, 2012. 
Gupta, Anamika:'Feasibility of Supercritical Carbon Dioxide as A Drilling Fluid for Deep Underbalanced Drilling Operations" MS Thesis -The Craft and Hawkins Department of Petroleum Engineering, Louisiana State University,2006

Gurney, K. R., Mendoza, D., Zhou, Y., Fischer, M., de la Rue du Can, S., Geethakumar, S., \& Miller, C. "The Vulcan Project: High resolution fossil fuel combustion $\mathrm{CO}_{2}$ emissions fluxes for the United States". Environ. Sci. Technol., 43. doi: 10.1021/es900,806c (2009).

Hill, A.C. and Thomas G.W., 1985,"A new approach for simulating complex fractured reservoirs", SPE 13537, SPE Reservoir Simulation Symposium, Anaheim, CA, February 10-13.

Hudson, J., Civan, F., Michel, V. G., Devegowda, D., Sigal, R.” Modeling multiple-porosity transport in gas-bearing shale formations" SPE-153535, SPE Latin American and Caribbean Petroleum Engineering Conference ,Mexico City, Mexico, SPE, April 16-18,2012

Hudson, J. "Quad-porosity model for description of gas transport in shale-gas reservoirs". MS thesis, Oklahoma U., Norman, Oklahoma, 2011.

Ikewun, P., Ahmadi, M., "Production Optimization and Forecasting of Shale Gas Wells Using Simulation Models and Decline Curve Analysis" SPE 153914, Western Regional Conference, Bakersfield, California, 19-23 March, 2012

Ilk, D., Rushing, J.A., Blasingame, T.A., "Integration of Production Analysis and Rate-Time Analysis via Parametric Correlations : Theoretical Considerations and Practical Applications", SPE 140556, SPE Hydraulic Fracturing Conference, The Woodlands, Texas, 24-26 January,2011.

Jakumeit , J., Herdy ,M., and Nitsche ,M.:” Parameter optimization of the sheet metal forming process using an iterative parallel Kriging algorithm” Structural and Multidisciplinary Optimization, 29(6):498$507,2005$.

Javadpour, F. "Nanopores and apparent permeability of gas flow in mudrocks (shales and siltstone)". J. of Canadian Petroleum Tech., v. 48, pp 16-21(2009)

Jurecka F." Robust Design Optimization Based on Meta modeling Techniques” Technische Universität München (2007) 
Johnson, N.L., Currie, S.M., Ilk, D., Blasingame, T.A., "A Simple Methodology for Direct Estimation of Gas-in-place and Reserves Using Rate-Time Data" SPE 123298, Rocky Mountain Technology Conference, Denver, Colorado, 14-16 April,2009.

Kalantari-Dahaghi .A, Esmaili.S, Mohaghegh. S:"Fast Track Analysis of Shale Numerical Models" SPE 162699, Canadian Unconventional Resources Conference held in Calgary, Alberta, Canada, 30 October-1 November 2012.

Kang, S.M., Fathi E., Ambrose R.J., Akkutlu I.Y., and Siga, R.F.," Carbon Dioxide Storage Capacity of Organic-Rich Shales” SPE Journal, Volume 16, Number 4, December 2011.

Katsube, T.J.: "Shale Permeability and Pore-Structure Evolution Characteristics," Research 2000 E15, Geological Survey of Canada, Ottawa, 2000.

Kazemi, H."Pressure transient analysis of naturally fractured reservoirs with uniform fracture distribution” SPE Journal, 9(4):451-462 (1969)

Kennedy, Robert L., Knecht, William N., Georgi , Daniel T.:" Comparisons and Contrasts of Shale Gas and Tight Gas Developments, North American Experience and Trends" SPE 160855 SPE Saudi Arabia Section Technical Symposium and Exhibition held in Al-Khobar, Saudi Arabia, 8-11 April 2012.

Krishnamurthy, T.:" Response surface approximation with augmented and compactly supported radial basis functions" In Proceedings of the 44th AIAA/ASME/ ASCE/AHS/ASC Structures, Structural Dynamics, and Materials Conference, Norfolk, VA, USA, April 2003.

KunzlePh.,:"VehiclaeControl With Neural Networks" GameDev.net

http://archive.gamedev.net/archive/reference/articles/article1988.html

Lee. J, Sidle R, "Gas Reserve Estimation in Resource Plays" SPE Economics \&Management Volume 2, Number 2, pp. 86-91, 2010.

Lewis, R. et al.: "New Evaluation Techniques for Gas Shale Reservoirs," Schlumberger paper for 2004 Reservoir Symposium.

Liner C. L.," Carbon capture and sequestration: overview and offshore aspects" in Proceedings of the Offshore Technology Conference (OTC '10), pp. 3511-3514, May 2010. 
Li,J, Guo B, Ling K,; Case Studies Suggest Heterogeneity is a Favorable Characteristics of Shale Gas Reservoirs" SPE 162702 SPE Canadian Unconventional Resources Conference held in Calgary, Alberta, Canada, 30 October-1 November 2012.

McCord Nelson, M., and Illingworth, W.T. "A Practical Guide to Neural Nets", Addison-Wesley Publishing, Reading, MA 1990.

Mongalvy, V., E. Chaput, S. Agarwal and L. Lu: "A New Methodology for Shale Reservoir Performance," paper SPE 144154 prepared for presentation at the 2011 Americas Unconventional Gas Conference held in the Woodlands, TX, 14-16 June.

Mohaghegh, S.D;" Reservoir modeling of shale formations" Journal of Natural Gas Science and Engineering, Volume 12, May 2013, Pages 22-33

Mohaghegh S.D:" Virtual Intelligence and Its Application in Petroleum Engineering" Distinguish author series, Journal of Petroleum Technology, September 2000.

Mattar, L., Gault, B., Morad, K., Clarkson, C.R., Freeman, C.M., Ilk, D., Blasingame, T.A.” Production Analysis and Forecasting of Shale Gas Reservoirs: Case History-Based Approach" SPE 119897, Shale Gas Production Conference, Fort Worth, Texas, November16-18, 2008.

Mfrac-Meyer Fracturing Software, a Baker-Hughes Company, www.mfrac.com

National Park Service (NPS) (Cartographer). (2009). World Physical Map. Retrieved from http://goto.arcgisonline.com/maps/World_Physical_Map

Myers, R. H. and Montgomery, D. C.:" Response Surface Methodology: Process and Product Optimization Using Designed Experiments” JohnWiley\& Sons, New York,NY, USA, 2nd edition, 2002

Nobakht, M., Clarkson, C.R., "A new analytical method for analyzing production data from shale gas reservoirs exhibiting linear flow: constant pressure production" SPE Reservoir Evaluation and Engineering Journal, 370-384. June 2012.

Nobakht, M., Mattar, L. "Analyzing production data from unconventional gas reservoirs with linear flow and apparent skin” Journal of Canadian Petroleum Technology, 52-59. January 2012. 
Nobakht, M., Mattar, L., Moghadam, S., Anderson, D.M., "Simplified Yet Rigorous Forecasting of Tight/Shale Gas Production in Linear Flow", SPE 133615, Western Regional Conference, Anaheim, CA, May 27-29, 2010.

Nuttall , Brandon C., Eble, Cortland F., Drahovzal „James A.,Bustin, R. Marc;” Analysis of Devonian Black Shales in Kentucky for Potential Carbon Dioxide Sequestration and Enhanced Natural Gas Production” Kentucky Geological Survey, Final Report, December 30, 2005

Orr F. M.:"Storage of carbon dioxide in geologic formations" Journal of Petroleum Technology, vol. 56, no. 9, pp. 90-97 (2004).

Petrusak R., Riestenberg D., Goad P.: "World class CO2 sequestration potential in saline formations, oil and gas fields, coal, and shale: the US southeast regional carbon sequestration partnership has it all" in Proceedings of the SPE International Conference on CO2 Capture, Storage, and Utilization, pp. 136-153, November 2009.

Polczer, S.: "Shale Expected to Supply Half of North America's Gas," Calgary Herald, April 9, 2009.

Rubin, B: "Accurate Simulation of Non-Darcy Flow in Stimulated Fracture Shale Reservoirs," SPE 132293 prepared for presentation at the Western Regional Conference held in Anaheim, CA, May 27 29,2010

Rubin, B. "Accurate Simulation of Non-Darcy Flow in Stimulated Fractured Shale Reservoirs" Paper SPE 132093, Western Regional Meeting, Anaheim, CA, May 27-29, 2010.

Rossen, R.H., "Simulation of naturally fractured reservoir with semi-implicit source terms" SPE Journal, 201-210, June 1977,

Saidi, A.M."Simulation of naturally fractured reservoirs" SPE 12270, Reservoir Simulation Symposium, San Francisco, CA, November 15-18, 1983.

Schepers , Karine C., Nuttall B. , Oudinot, Anne Y., Gonzalez, Reinaldo "Reservoir Modeling and Simulation of the Devonian Gas Shale of Eastern Kentucky for Enhanced Gas Recovery and $\mathrm{CO}_{2}$ Storage" SPE 126620, SPE International Conference on CO2 Capture, Storage, and Utilization, 2-4 November 2009, San Diego, California, USA 
Shabro, V., Torres-Verdin, C., and Javadpour, F."Numerical simulation of shale-gas production: from pore-scale modeling of slip-flow, Knudsen diffusion, and Langmuir desorption to reservoir modeling of compressible fluid" SPE 144355, North American Unconventional Gas Conference and Exhibition in the Woodlands, Texas, USA, June14-16, 2011

Shabro, V., Torres-Verdin, C., and Sepehrnoori, K. "Forecasting gas production in organic shale with the combined numerical simulation of gas diffusion in kerogen, Langmuir desorption from kerogen surfaces, and advection in nanopores" SPE-159259, Annual Technical Conference and Exhibition held in San Antonio, Texas, USA, October 8-10, 2012.

Sinayuç C. and Gümrah F.: "Modeling of ECBM recovery from amasracoalbed in Zonguldak Basin, Turkey" in Proceedings of the Canadian International Petroleum Conference, Alberta, Canada, 2008.

Soeder, D." Porosity and Permeability of Eastern Devonian Gas Shale" Society of Petroleum Engineers. Institute of Gas Technology.: http://www.pe.tamu.edu/wattenbarger/public_html/Selected_papers/-Shale\%20Gas/SPE15213.pdf, Accessed April 2010.

Suliman B., Meek R., Hull R., Bello H., Portis D., and Richmond P.” Variable Stimulated Reservoir Volume (SRV) Simulation: Eagle Ford Shale Case Study” SPE 164546, SPE Unconventional Resources Conference-USA held in The Woodlands, Texas, USA, 10-12 April 2013.

Stevens S. H., Spector D., and Riemer P., "Enhanced coalbed methane recovery using CO2 injection: worldwide resource and CO2 sequestration potential," in Proceedings of the $6^{\text {th }}$ International Oil \& Gas Conference and Exhibition in China (IOGCEC '98), pp. 489-501, Beijing, China, November 1998.

U.S. Department of Energy, Office of Fossil Energy and National Energy Technology Laboratory, 2009, "Modern Shale Gas Development in the United States: A Primer" prepared by Ground Water Protection Council and ALL Consulting (April 2009).

U.S. Energy Information Administration (EIA)" U.S. Crude Oil, Natural Gas, and Natural Gas Liquids Reserves” U.S. Department of Energy, Washington, DC (November 30, 2010).

U.S. Energy Information Administration (EIA) "Review of Emerging Resources: U.S. Shale Gas and Shale Oil Plays" prepared by INTEK Inc. for the EIA, U.S. Department of Energy, Washington, DC (July 2011). 
U.S. Census Bureau (Cartographer).” Nation, U.S., Current State and Equivalent National” (2010) Retrieved from http://www.census.gov/cgi-bin/geo/shapefiles2010/main.

U.S. Energy Information Administration (EIA) (Cartographer). (2011a). "Maps: Exploration, Resources, Reserves, and Production, Shapefiles for basin boundaries" Retrieved from ftp://www.eia.doe.gov/pub/oil_gas/natural_gas/analysis_publications/maps/maps.htm\#geodata.

U.S. Energy Information Administration (EIA) (Cartographer). (2011b). "Maps: Exploration, Resources, Reserves, and Production, Shapefiles for play boundaries" Retrieved from ftp://www.eia.doe.gov/pub/oil_gas/natural_gas/analysis_publications/maps/maps.htm\#geodata

U.S. Department of Energy-National Energy Technology Laboratory (2010)" Impact of the Marcellus Shale Gas Play on Current and Future CCS Activities"

http://www.netl.doe.gov/technologies/carbon_seq/refshelf/Marcellus_CCS.pdf

Wang, J. and Y. Liu: "Well Performance Modeling in Eagle Ford Shale Oil Reservoir," SPE 144427, Americas Unconventional Gas Conference held in the Woodlands, TX, 14-16 June. 2011

Warlick International, North American Unconventional Gas Market Report 2010, Edition 1 of 2, (2010).

Williams, G.J.J., Mansfield, M., MacDonald, D.G., and Bush, M.D. 2004. "Top-Down Reservoir Modeling" SPE 89974 presented at the 2004 SPE Annual Technical Conference and Exhibition, Houston, 26-29 September.

Williams, M.A. “Assessing Dynamic Reservoir Uncertainty: Integrating Experimental Design with Field Development Planning" 2006. SPE Distinguished Lecturer Series presentation given for Gulf Coast Section SPE, Houston, 23 March

Wilson, K. C., Durlofsky, L. J.” Computational Optimization of Shale Resource Development Using Reduced-Physics Surrogate Models" SPE 152946, SPE Western Regional Meeting, 21-23 March 2012, Bakersfield, California, USA

Xie .J,LeeS,WangZ,Wen, X.H:" Uncertainty Assessment of Production Performance for Shale Gas Reservoirs” IPTC 16866,International Petroleum Technology Conference, China, March 2013 
Yan. B, Wang. Y, Killough J. E.” Beyond Dual-Porosity Modeling for the Simulation of Complex Flow Mechanisms in Shale Reservoirs" SPE 163651, SPE Reservoir Simulation Symposium held in The Woodlands, Texas USA, 18-20 February 2013.

Zagorski, W. A., Bowman, D. C., Emery, M., \& Wrightstone, G. R. (2011). An Overview of Some Key Factors Controlling Well Productivity in Core Areas of the Appalachian Basin Marcellus Shale Play. Search and Discovery.

Ziarani, A. S., Aguilera, R.” Knudsen's permeability correction for tight porous media" Transport in Porous Media, Vol.91: 239-260. (2012)

Zhou W,GuptaS,Poe B, Spath J, Thambynayagam M;" Production Forecasting and Analysis for Unconventional Resources” IPTC 17176 ,International Petroleum Technology Conference ,Beijing, China, March 2013. 


\section{APPENDIX A -COMPARISON OF GAS PRODUCTION PROFILE FROM SIMULATOR AND DATA- DRIVEN PROXY MODEL FOR DIFFERENT TIME RESOLUTIONS (DAILY, MONTHLY \& ANNUAL)- PRODUCTION PHASE}

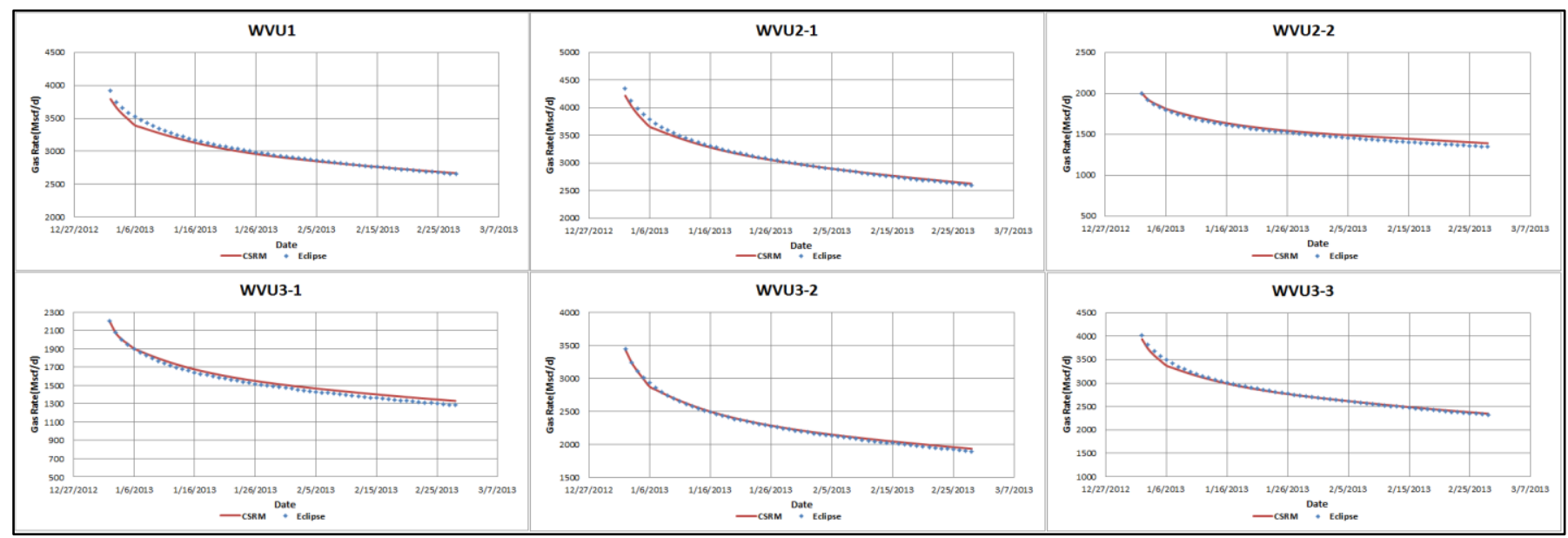

Figure A- 1 Comparison of gas production profile from simulator and Daily Data-driven proxy model-Run1 (Whole lateral)

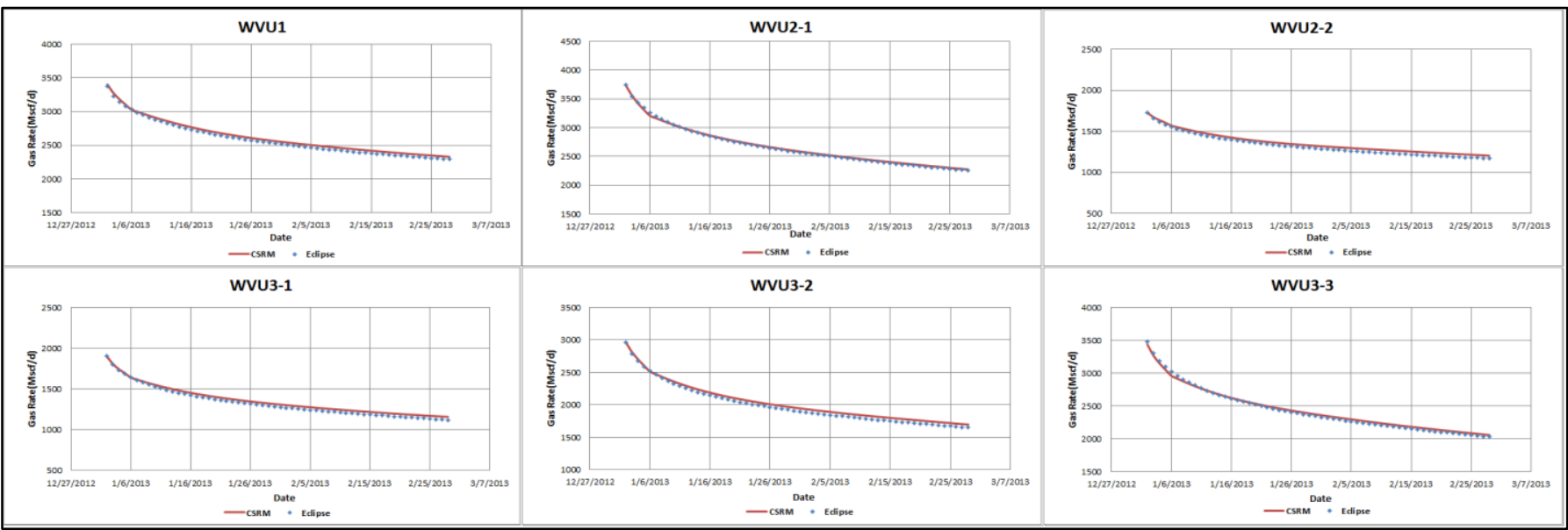

Figure A- 2 Comparison of gas production profile from simulator and Daily Data-driven proxy model-Run2 (Whole lateral)

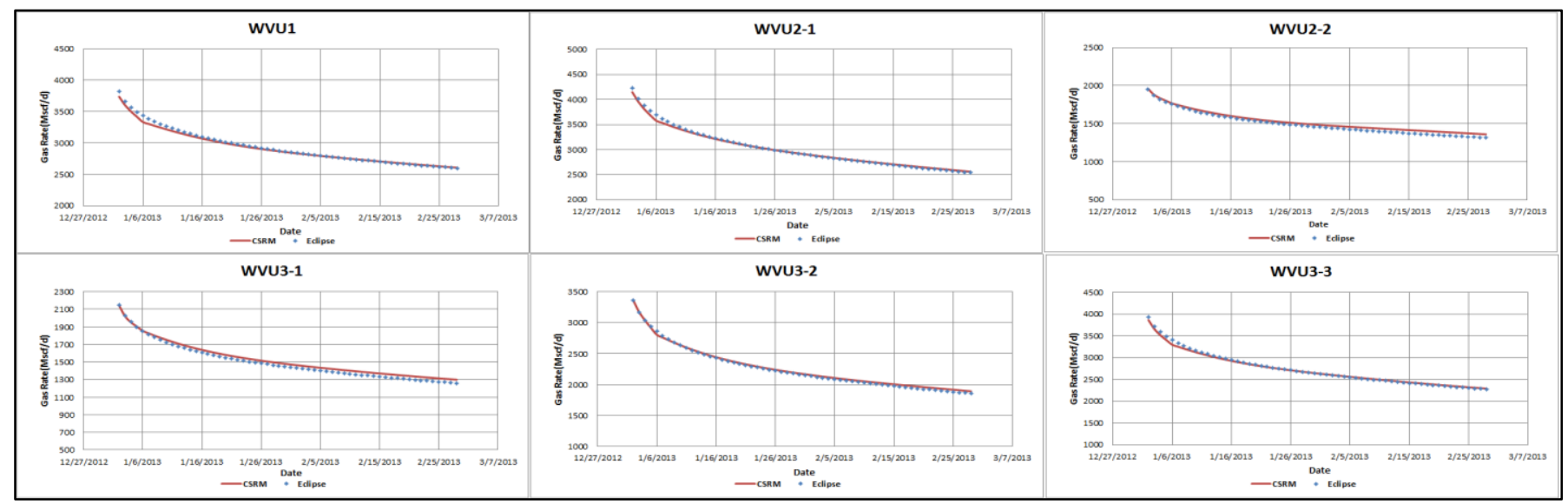

Figure A- 3 Comparison of gas production profile from simulator and Daily Data-driven proxy model-Run3 (Whole lateral) 


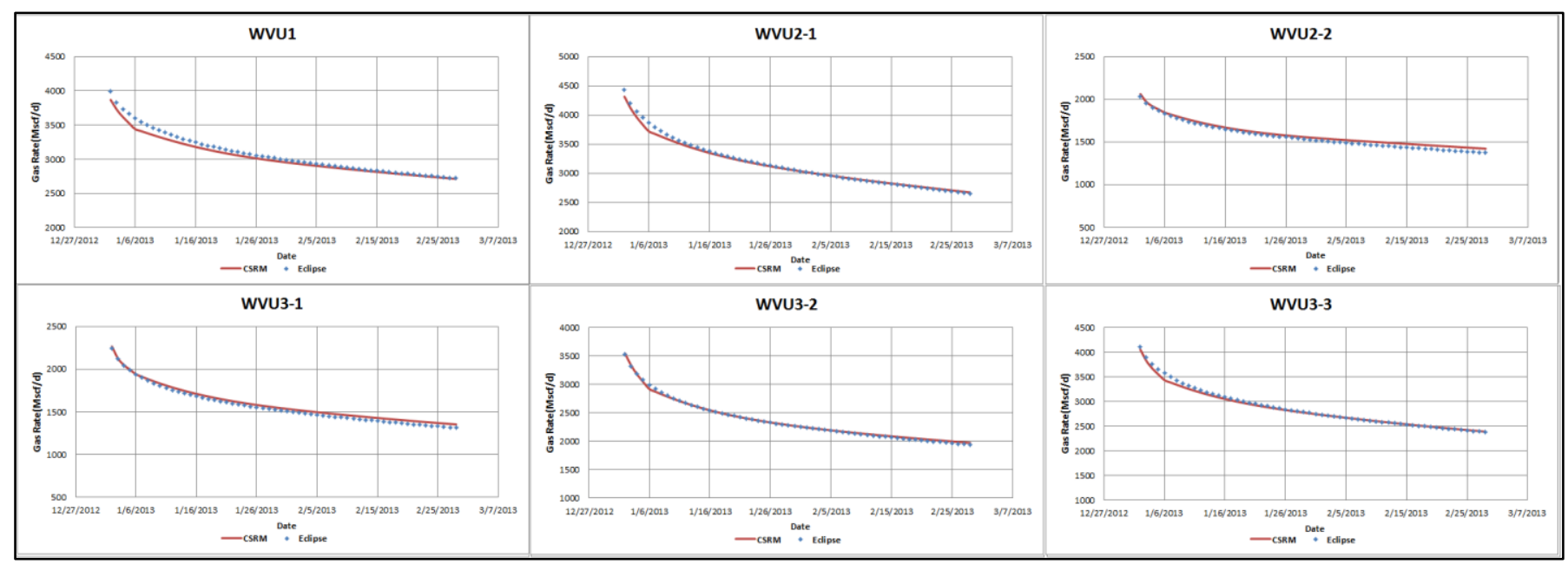

Figure A- 4 Comparison of gas production profile from simulator and Daily Data-driven proxy model-Run4 (Whole lateral)

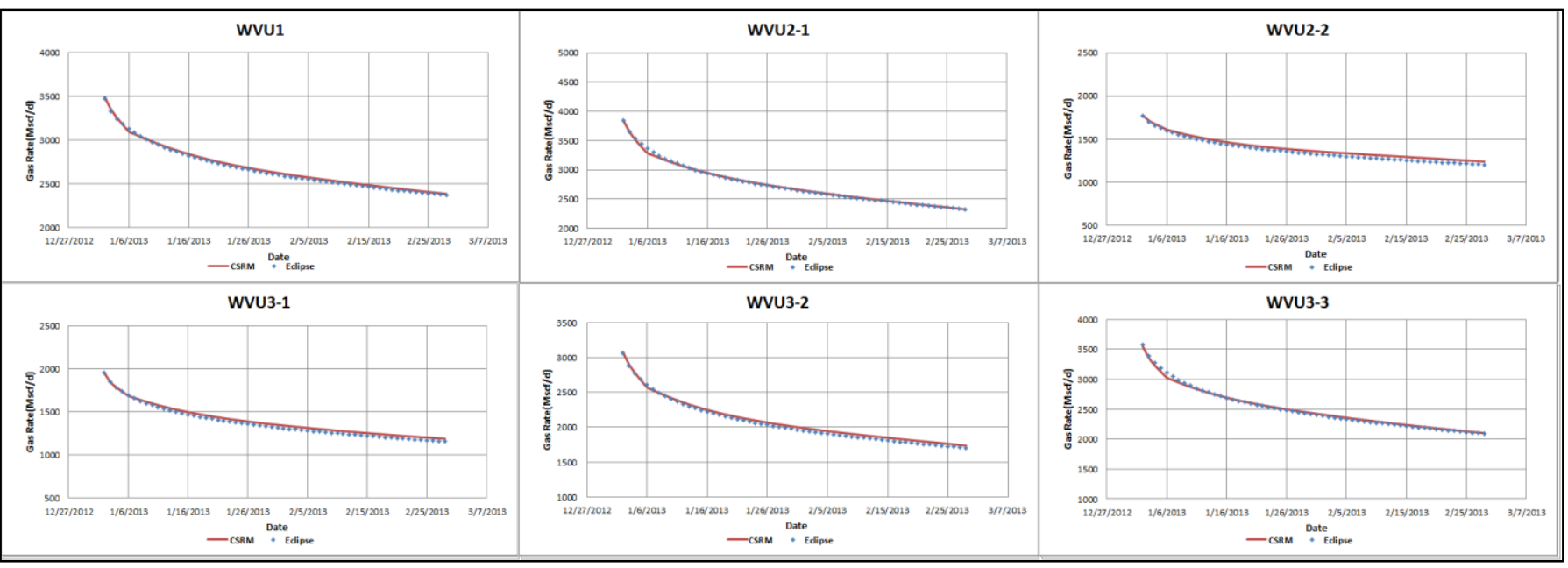

Figure A- 5 Comparison of gas production profile from simulator and Daily Data-driven proxy model-Run5 (Whole lateral)
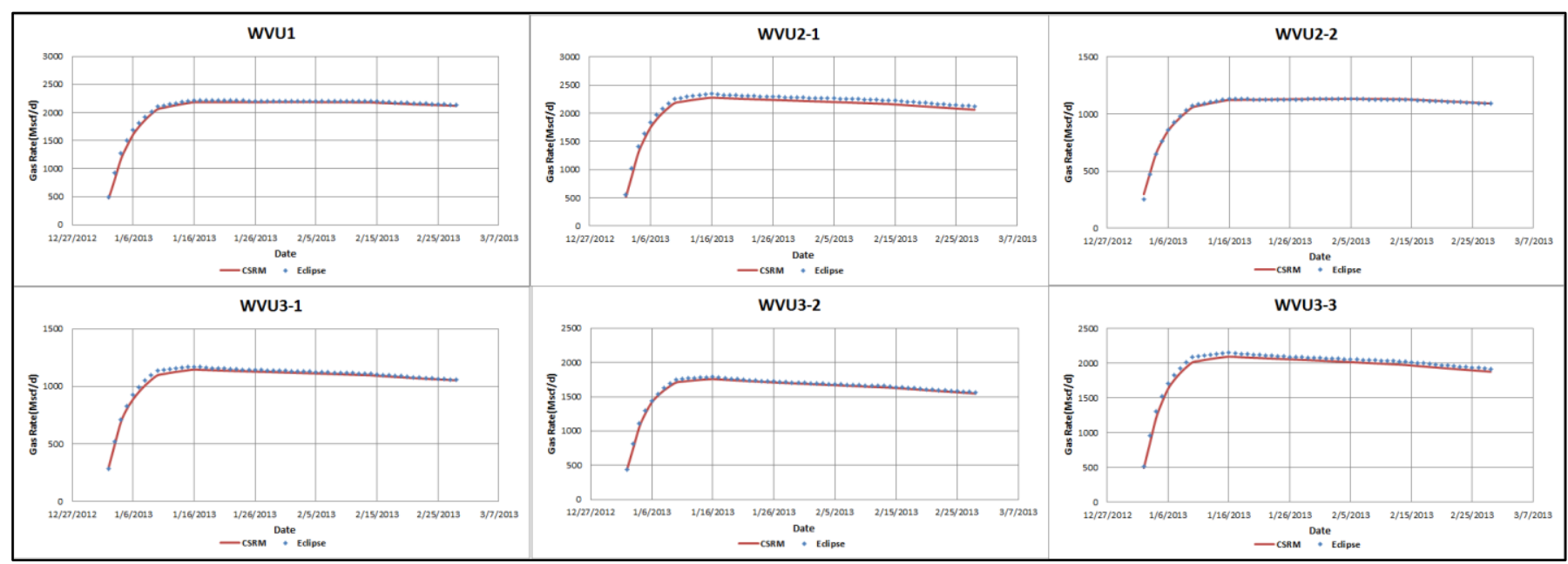

Figure A- 6 Comparison of gas production profile from simulator and Daily Data-driven proxy model-Run6 (Whole lateral) 


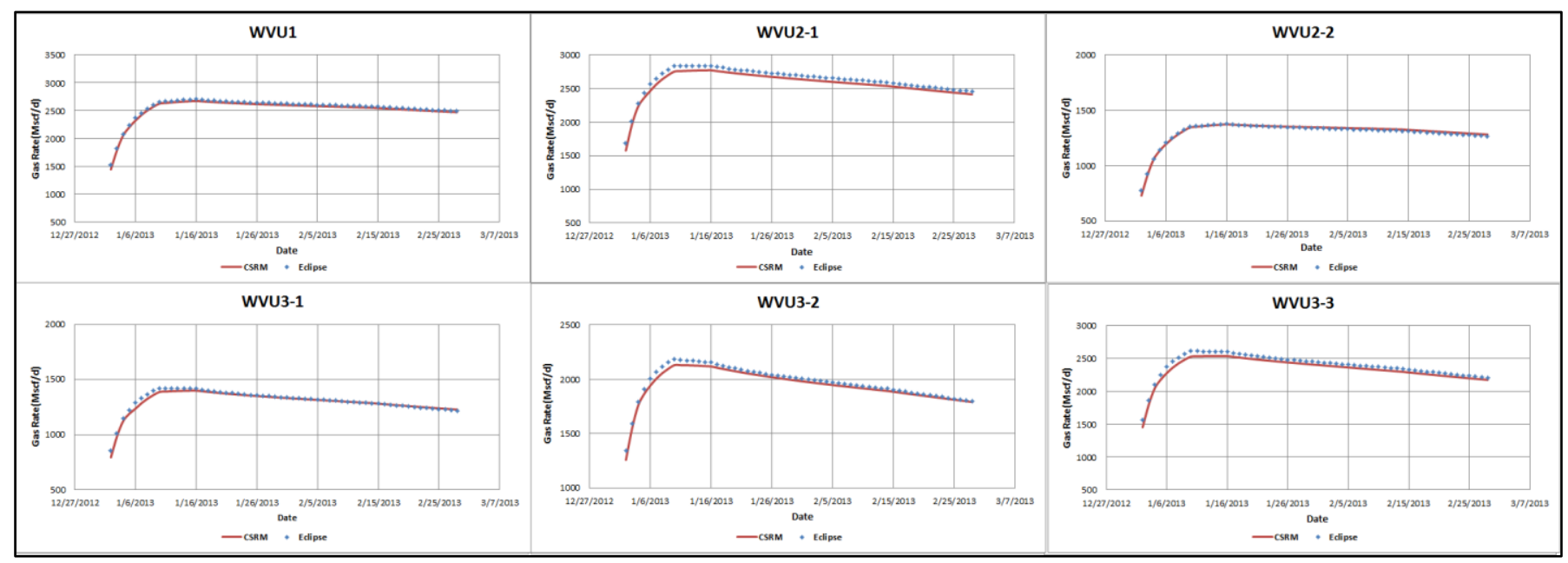

Figure A- 7 Comparison of gas production profile from simulator and Daily Data-driven proxy model-Run7 (Whole lateral)

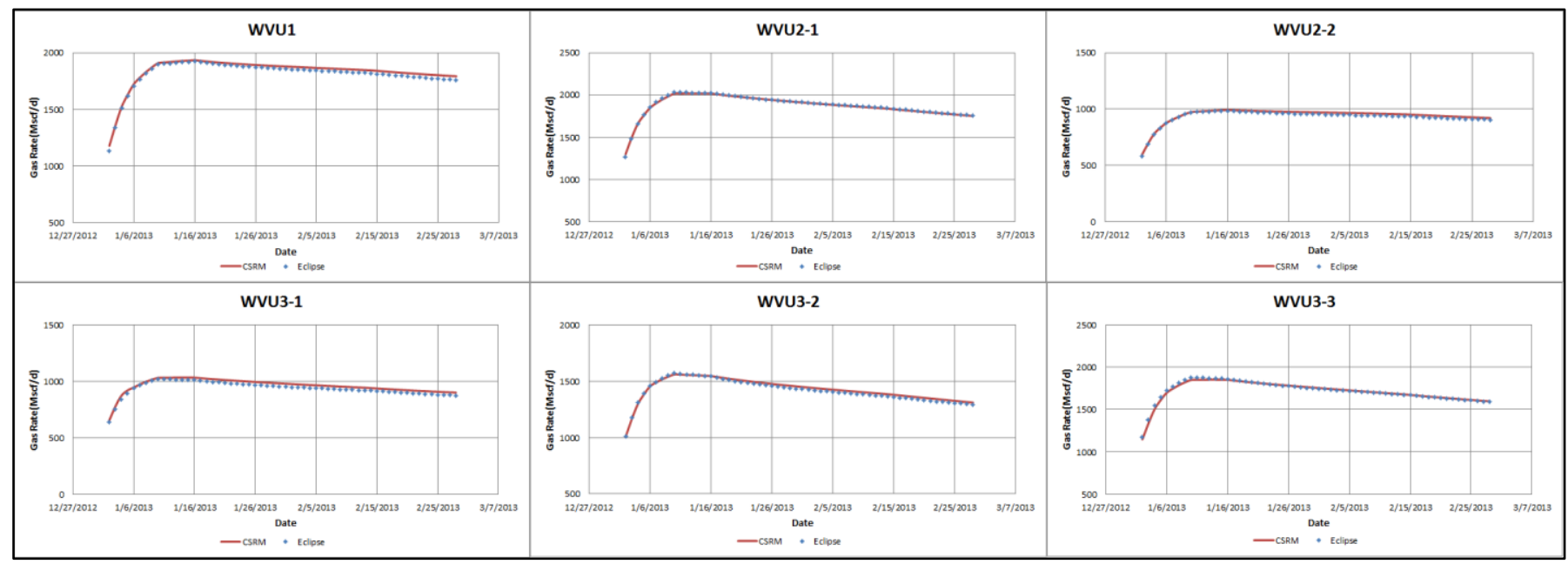

Figure A- 8 Comparison of gas production profile from simulator and Daily Data-driven proxy model-Run8 (Whole lateral)

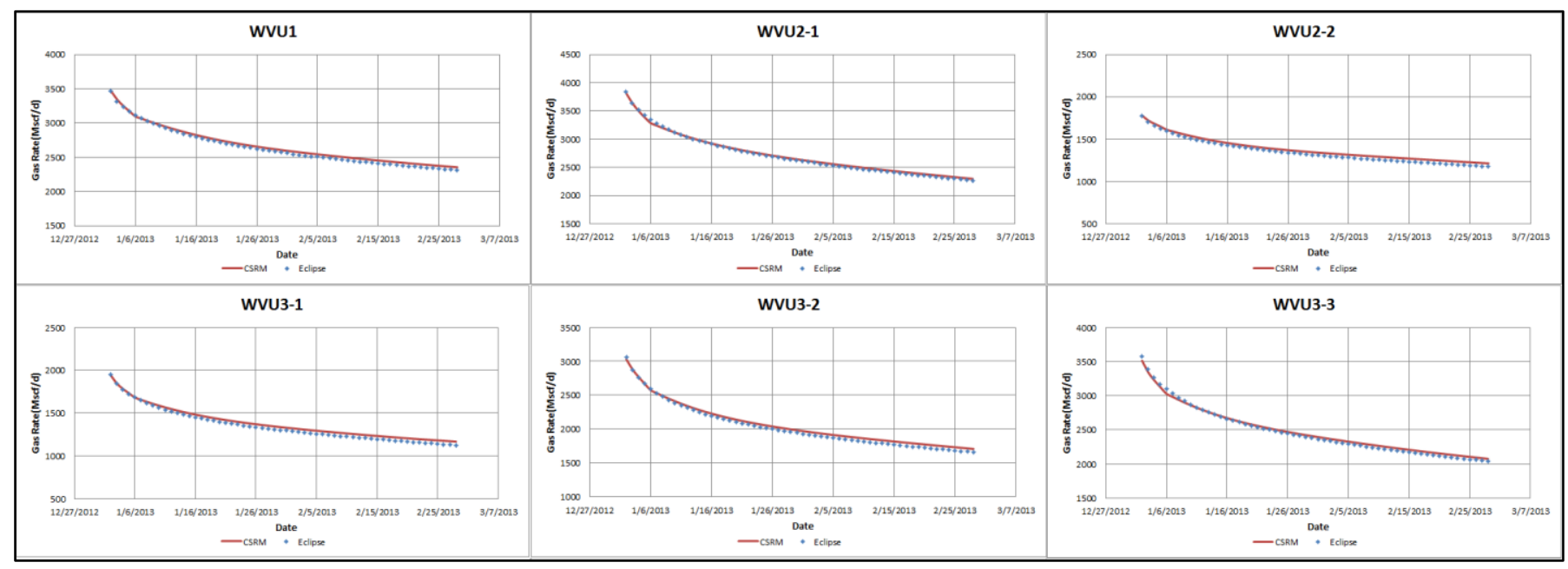

Figure A- 9 Comparison of gas production profile from simulator and Daily Data-driven proxy model-Run9 (Whole lateral) 


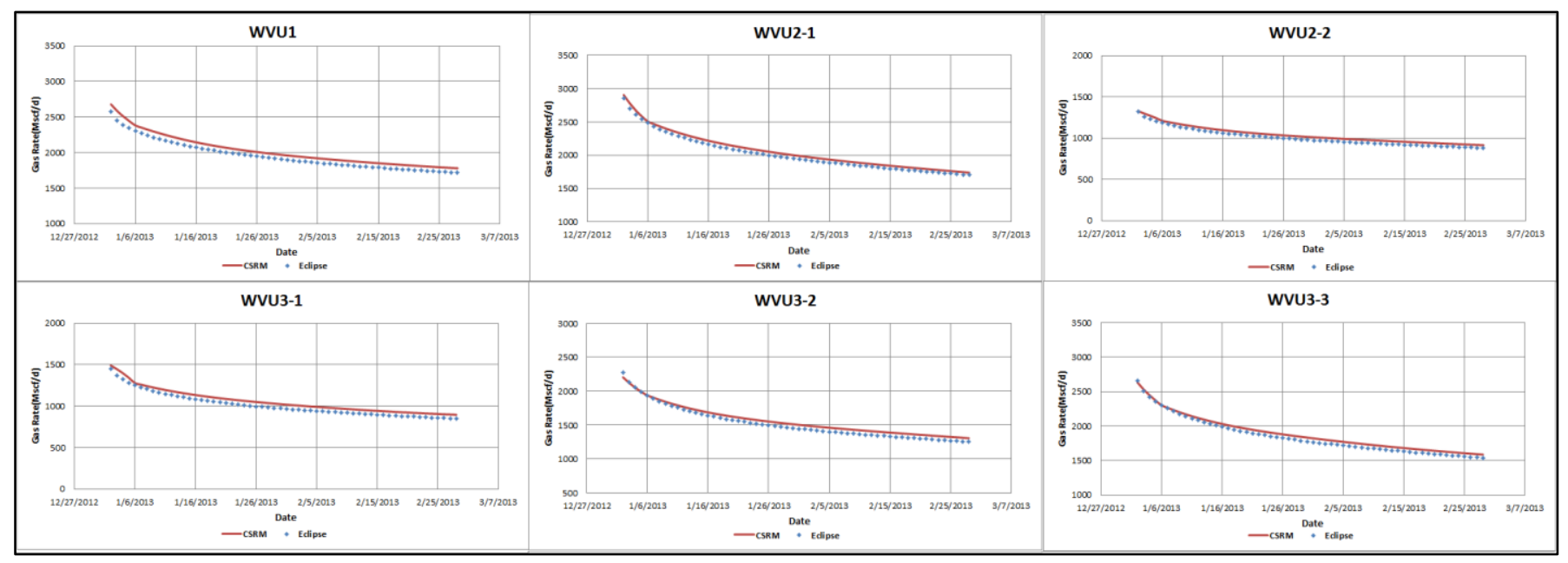

Figure A-10 Comparison of gas production profile from simulator and Daily Data-driven proxy model-Run10 (Whole lateral)

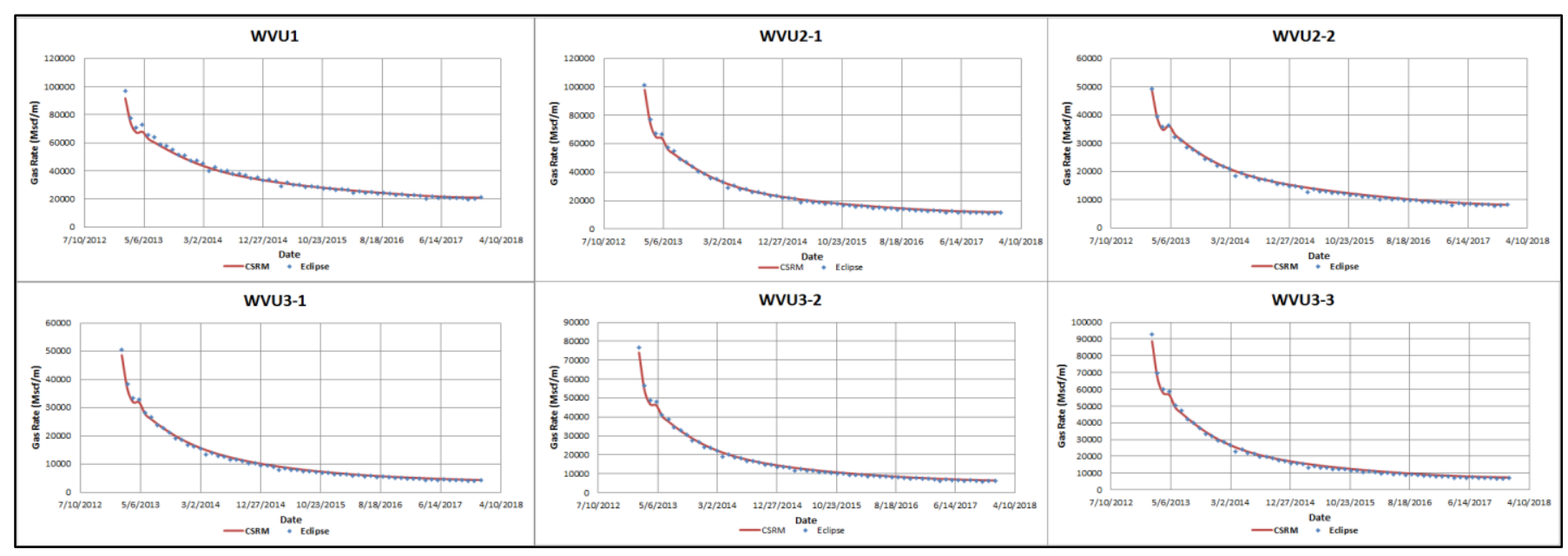

Figure A- 11 Comparison of gas production profile from simulator and Monthly Data-driven proxy model-Run1 (Whole lateral)

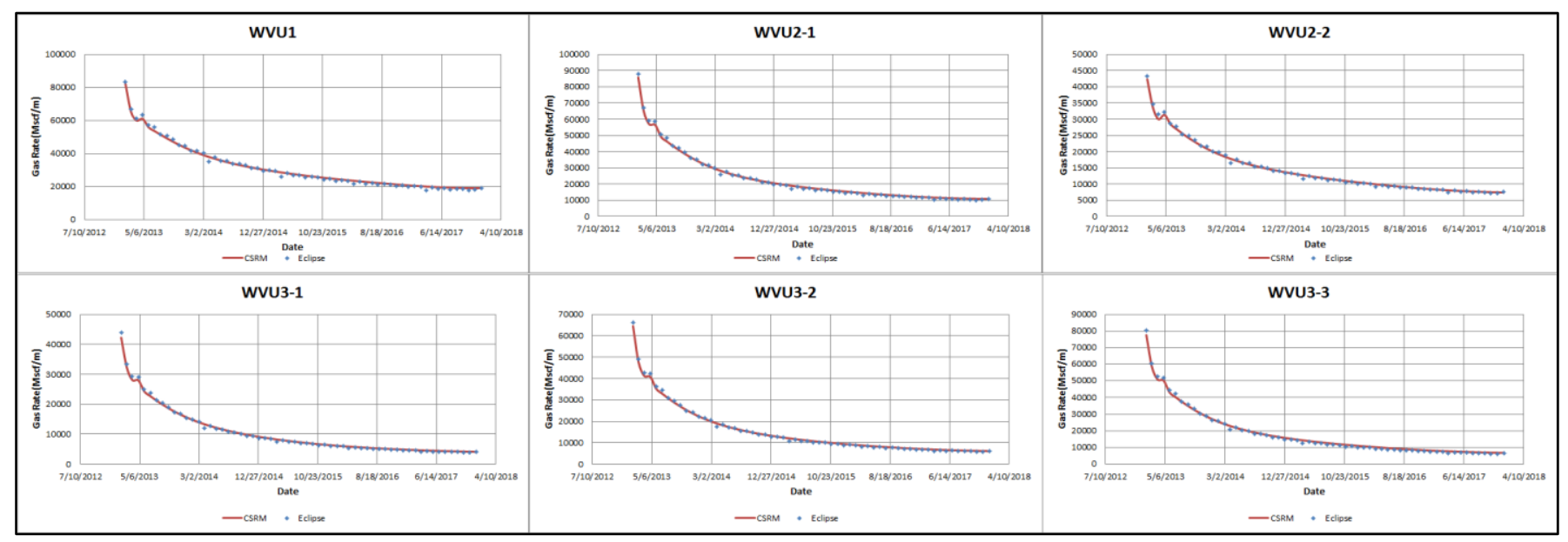

Figure A- 12 Comparison of gas production profile from simulator and Monthly Data-driven proxy model-Run2 (Whole lateral) 


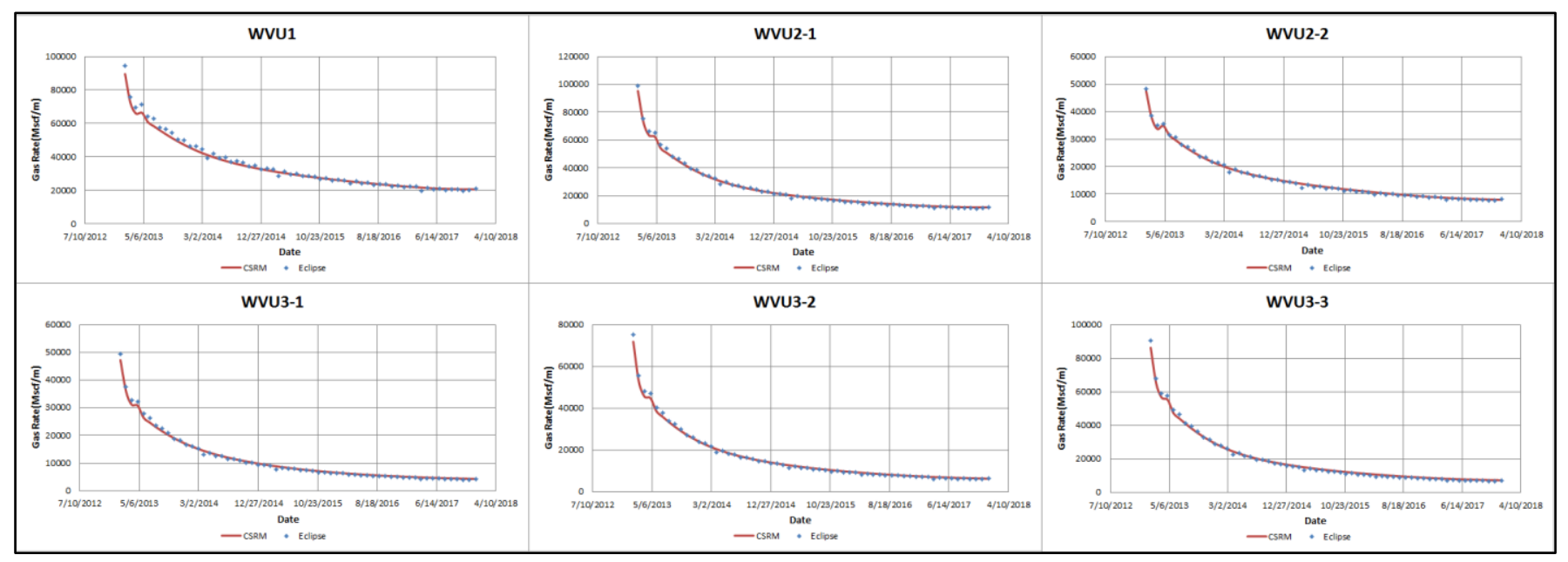

Figure A- 13 Comparison of gas production profile from simulator and Monthly Data-driven proxy model-Run3 (Whole lateral)

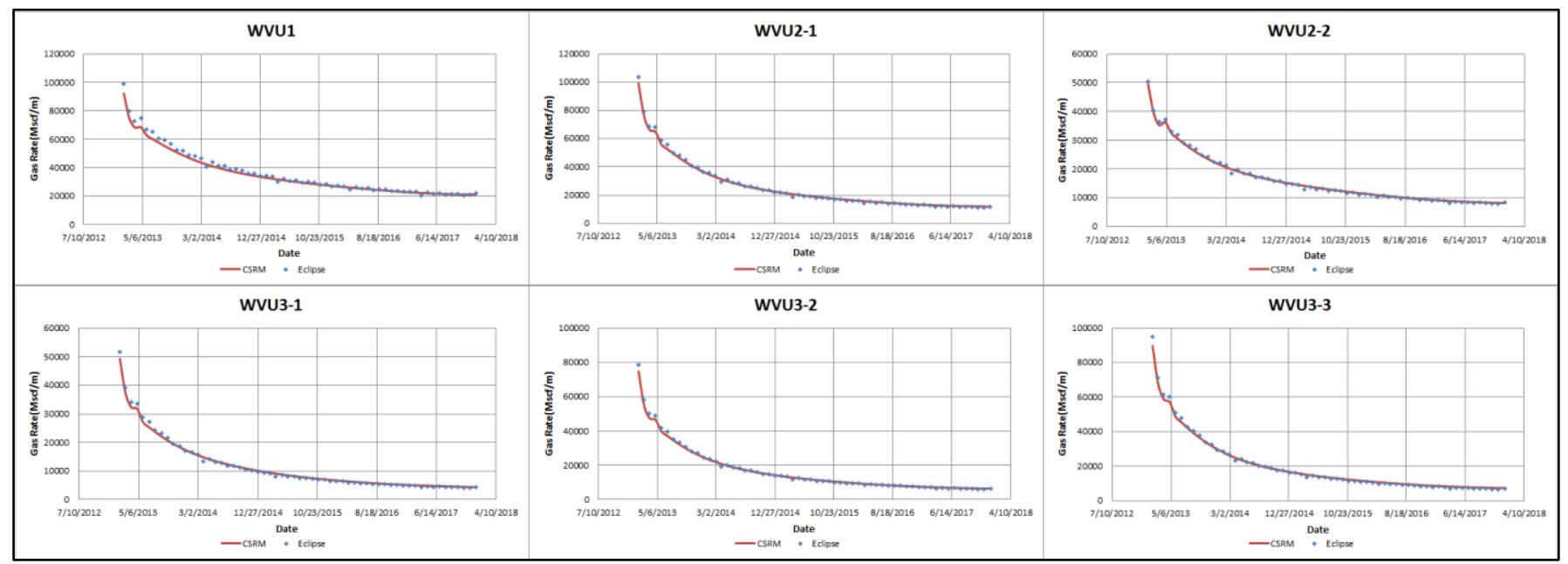

Figure A- 14 Comparison of gas production profile from simulator and Monthly Data-driven proxy model-Run4 (Whole lateral)

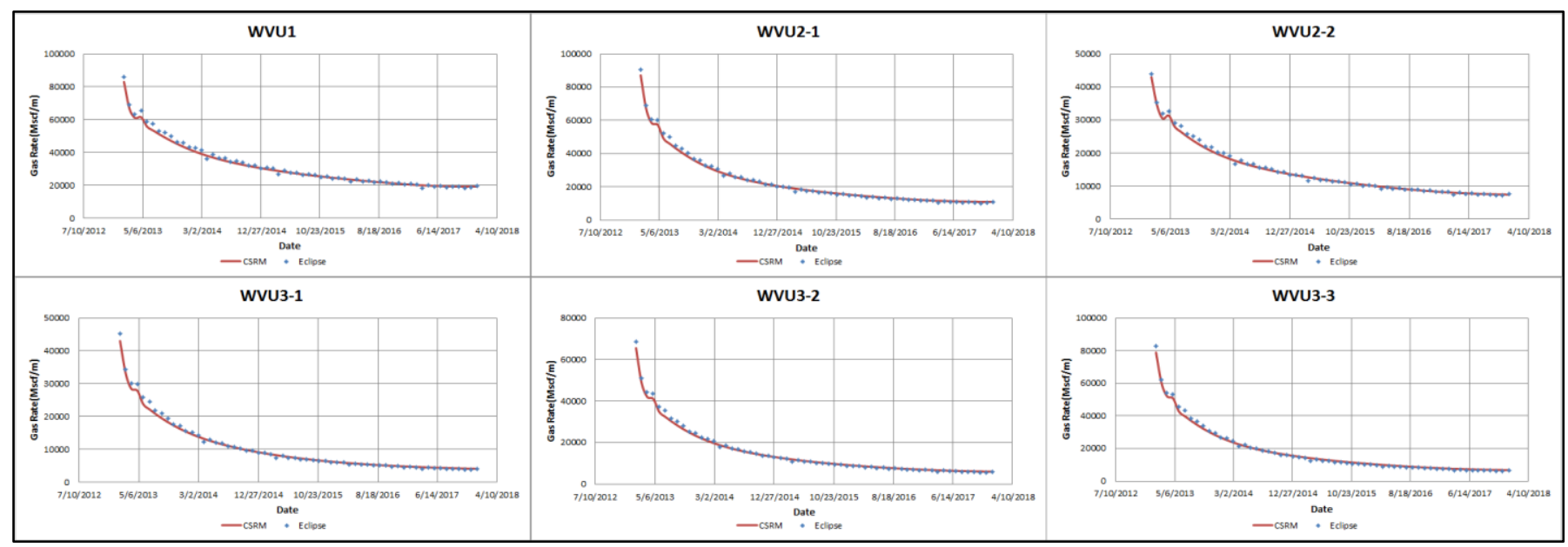

Figure A- 15 Comparison of gas production profile from simulator and Monthly Data-driven proxy model-Run5 (Whole lateral) 


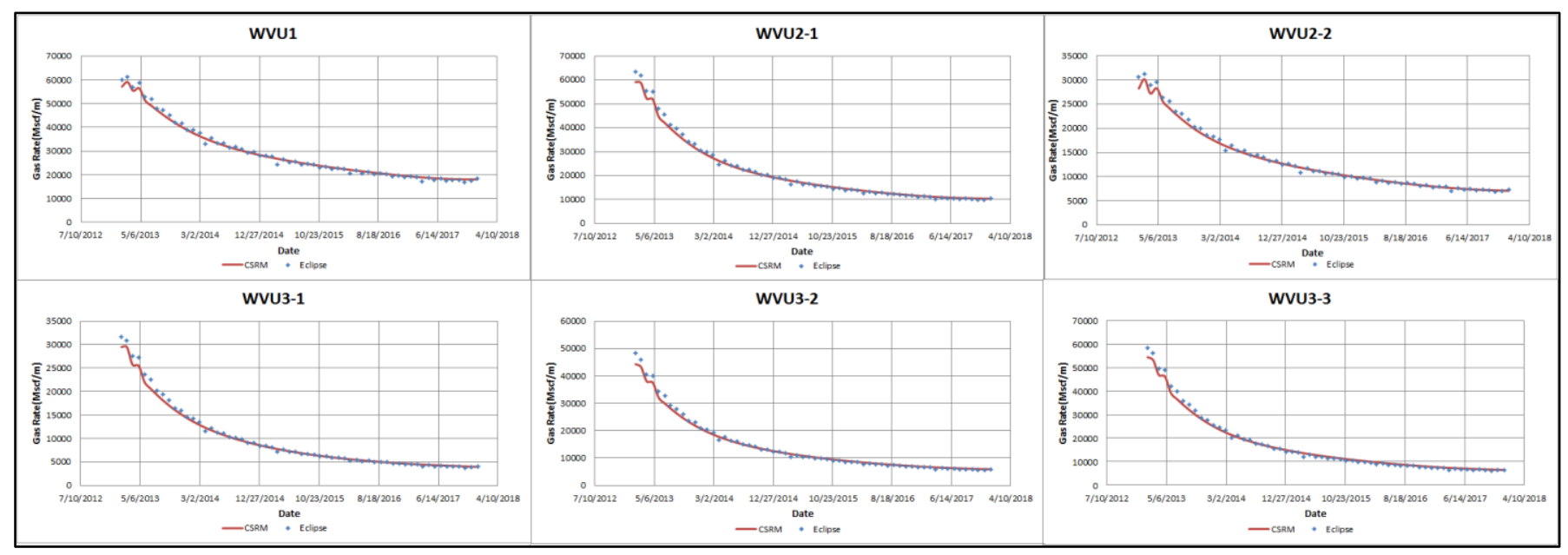

Figure A- 16 Comparison of gas production profile from simulator and Monthly Data-driven proxy model-Run6 (Whole lateral)
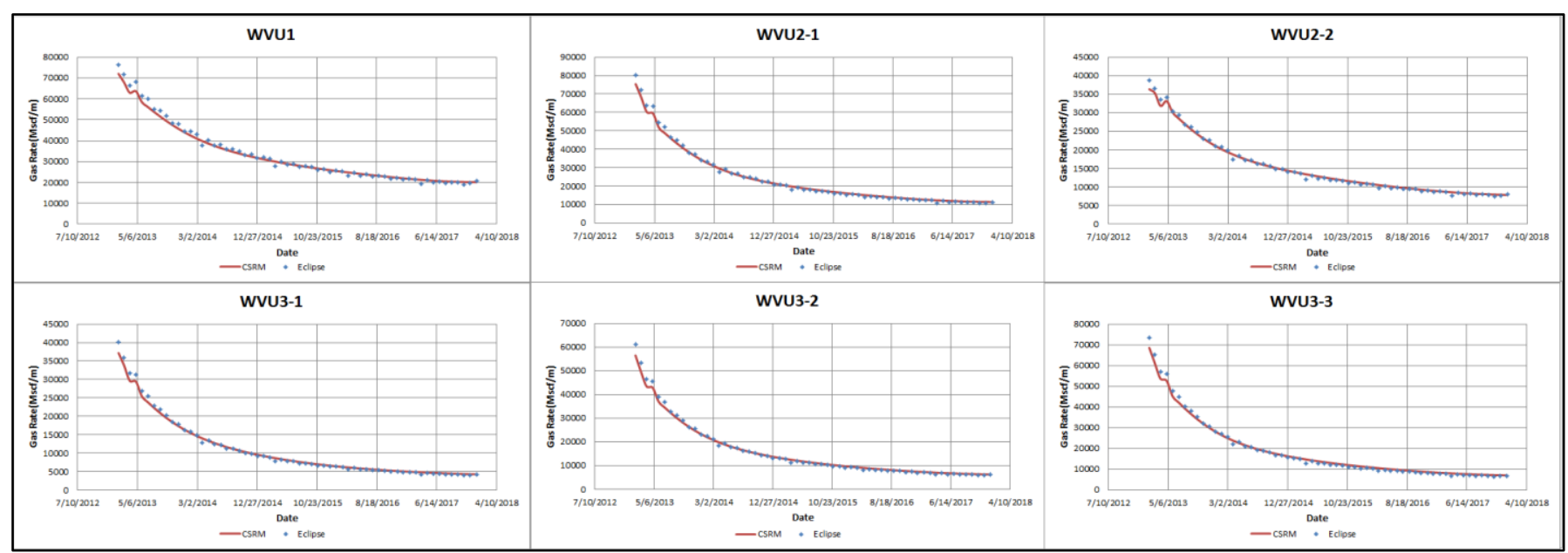

Figure A- 17 Comparison of gas production profile from simulator and Monthly Data-driven proxy model-Run7 (Whole lateral)
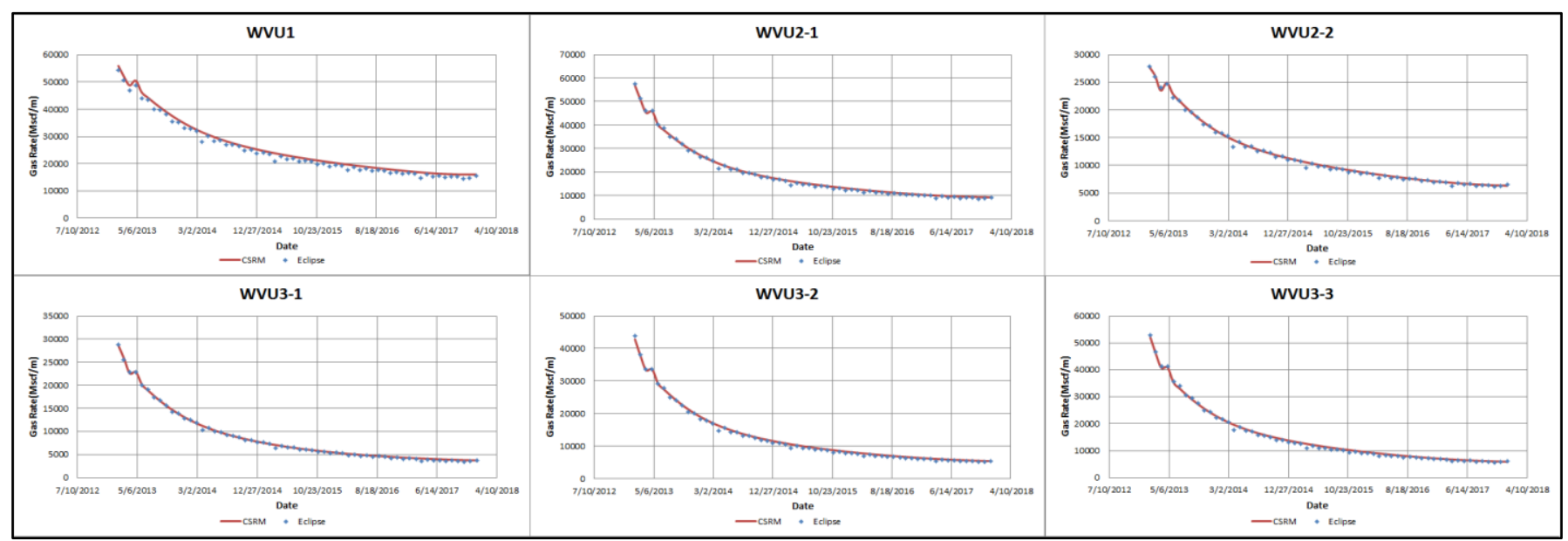

Figure A- 18 Comparison of gas production profile from simulator and Monthly Data-driven proxy model-Run8 (Whole lateral) 


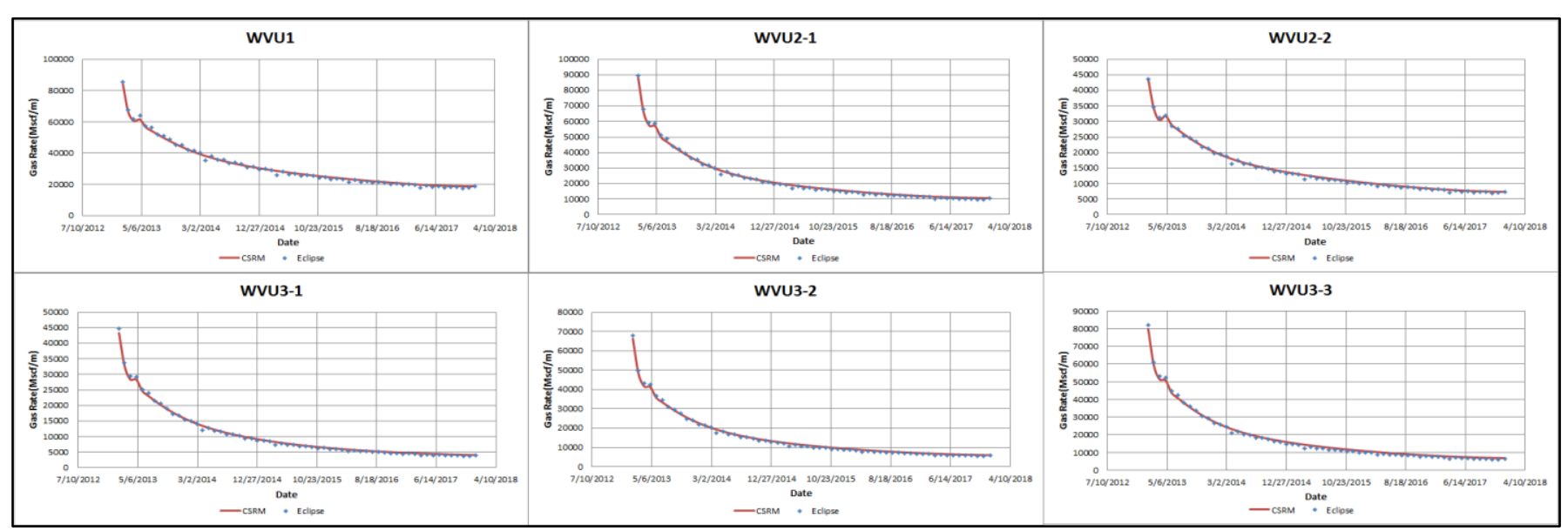

Figure A- 19 Comparison of gas production profile from simulator and Monthly Data-driven proxy model-Run9 (Whole lateral)

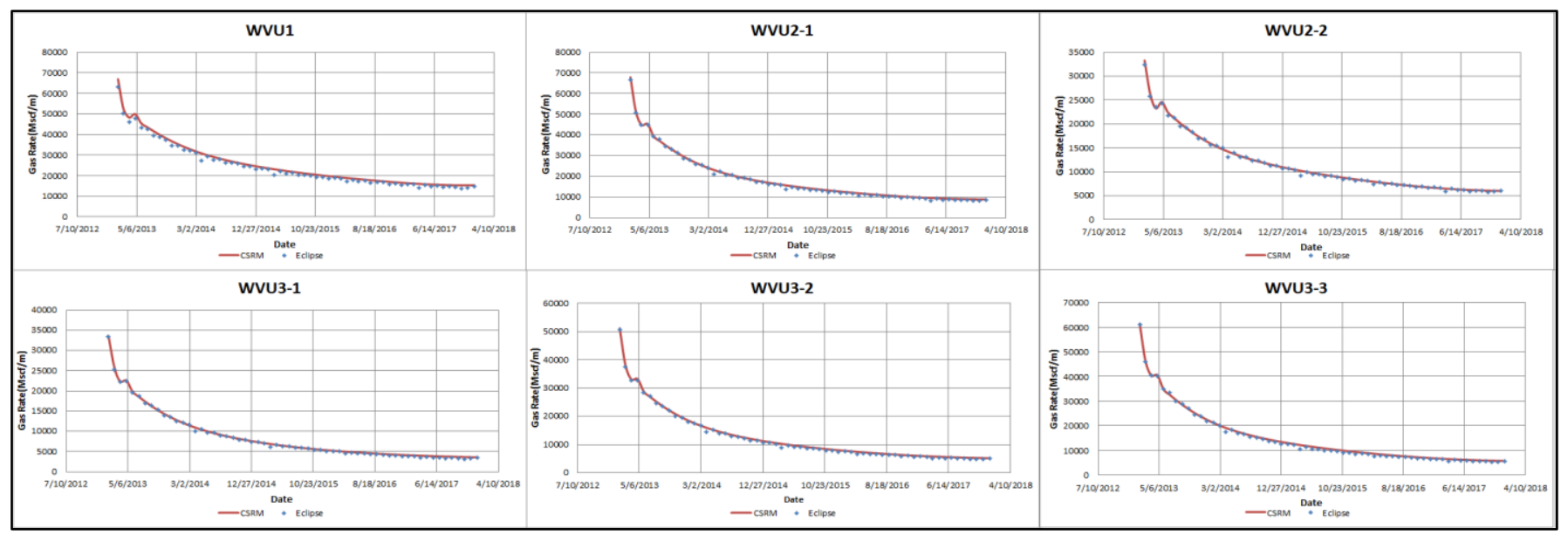

Figure A- 20 Comparison of gas production profile from simulator and Monthly Data-driven proxy model-Run10 (Whole lateral)

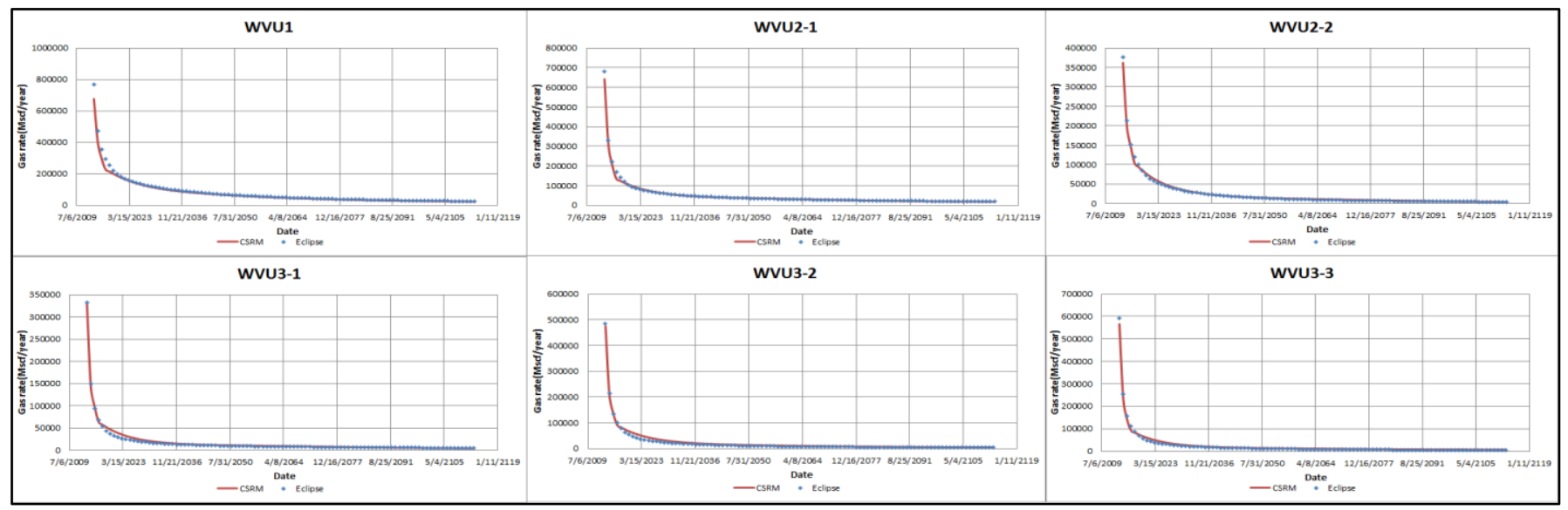

Figure A- 21 Comparison of gas production profile from simulator and Annual Data-driven proxy model-Run10 (Whole lateral) 


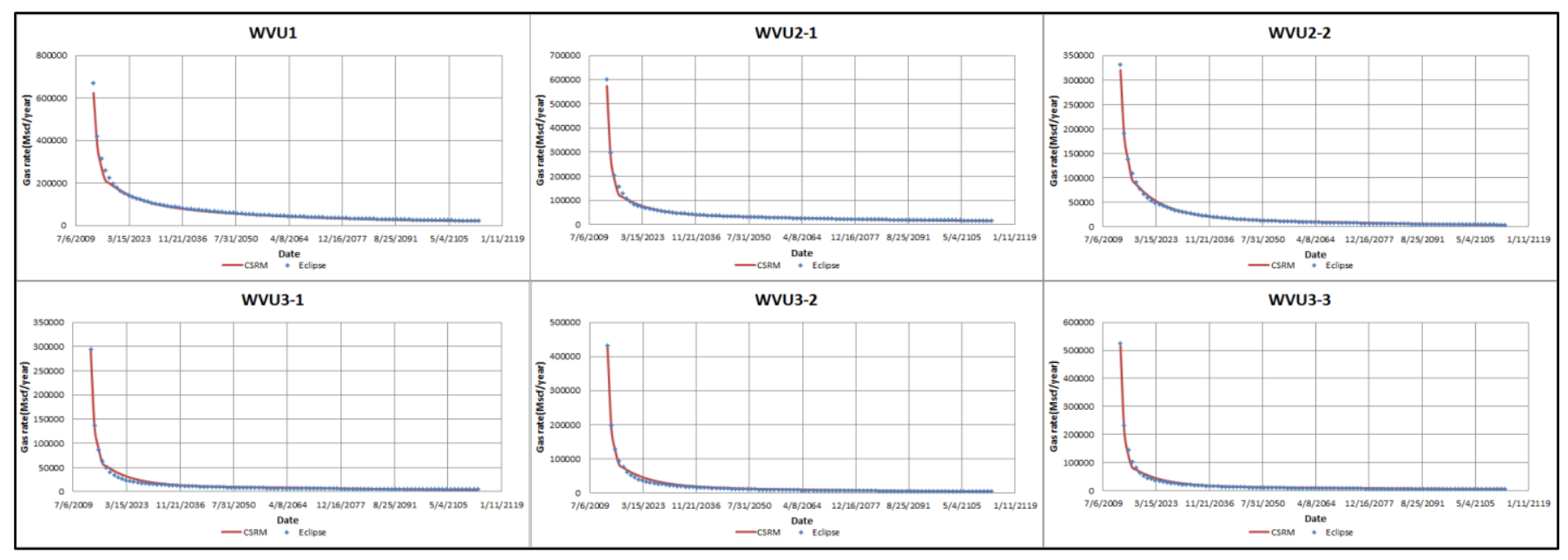

Figure A- 22 Comparison of gas production profile from simulator and Annual Data-driven proxy model-Run10 (Whole lateral)

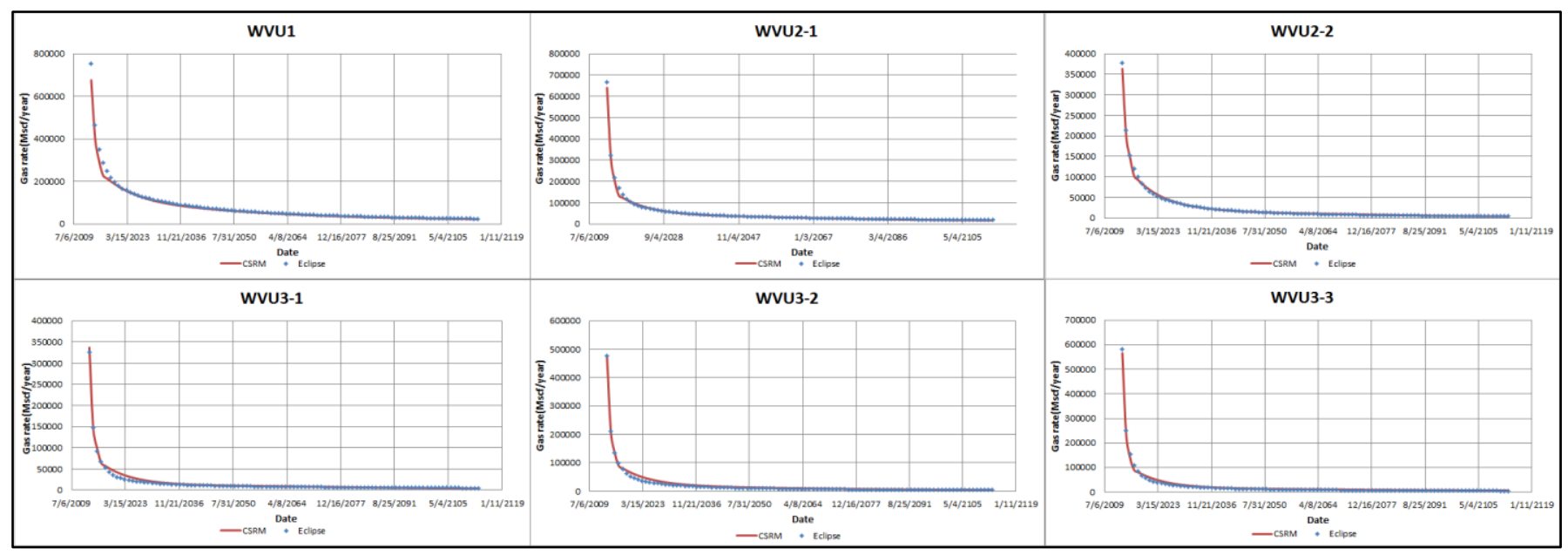

Figure A- 23 Comparison of gas production profile from simulator and Annual Data-driven proxy model-Run10 (Whole lateral)

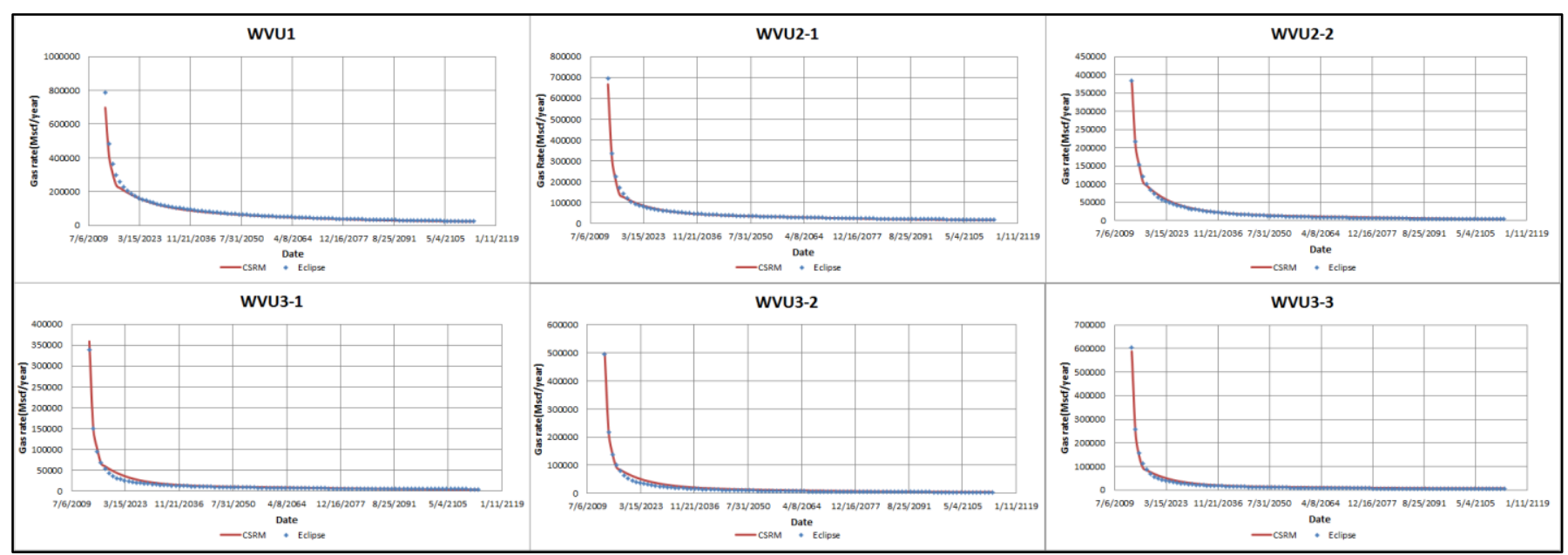

Figure A- 24 Comparison of gas production profile from simulator and Annual Data-driven proxy model-Run10 (Whole lateral) 


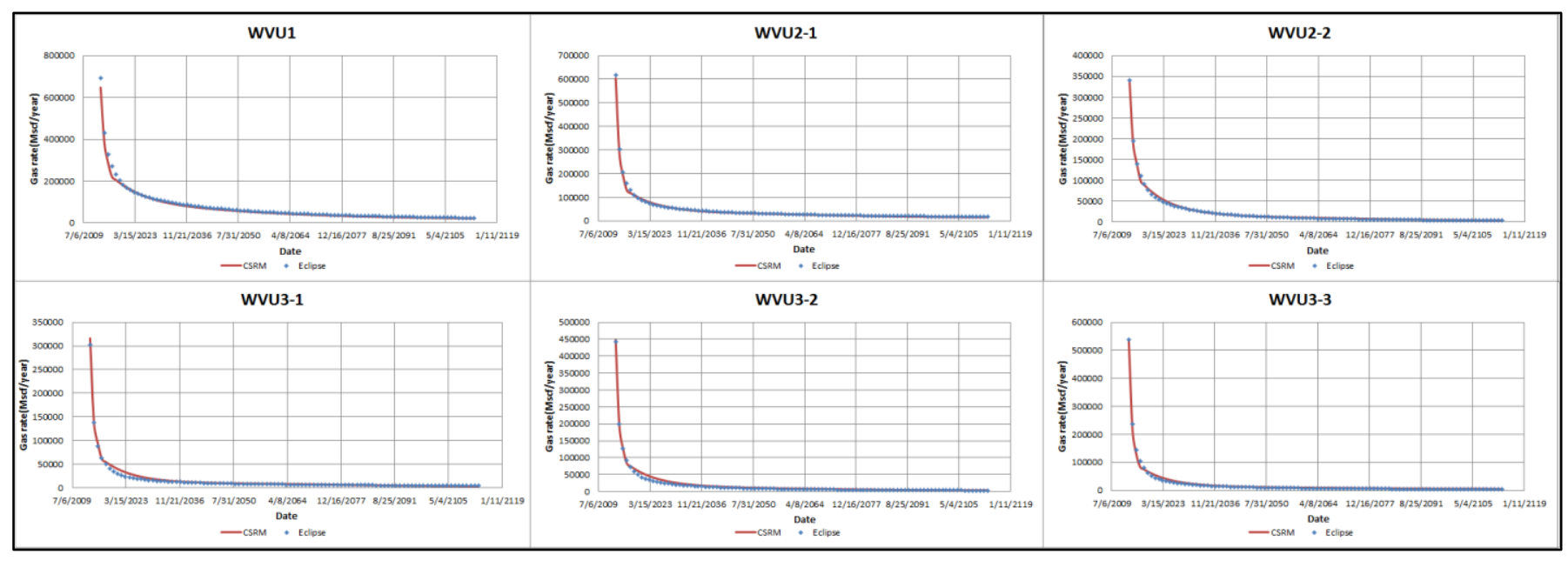

Figure A- 25 Comparison of gas production profile from simulator and Annual Data-driven proxy model-Run10 (Whole lateral)

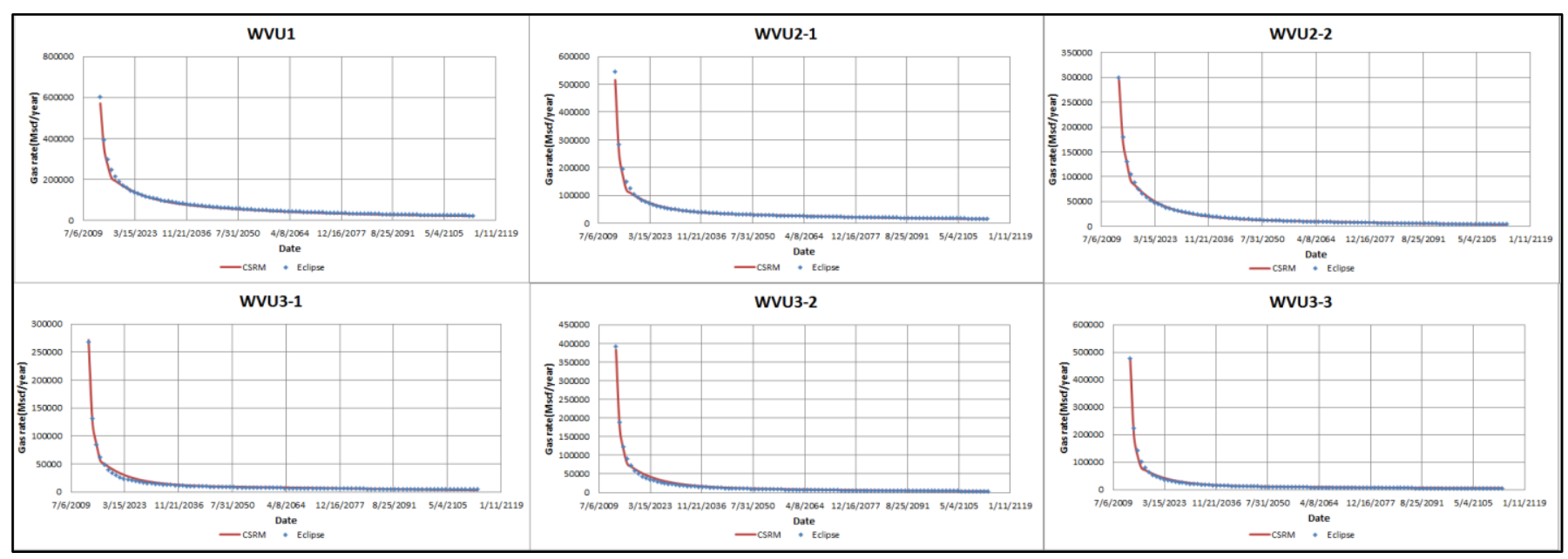

Figure A- 26 Comparison of gas production profile from simulator and Annual Data-driven proxy model-Run10 (Whole lateral)

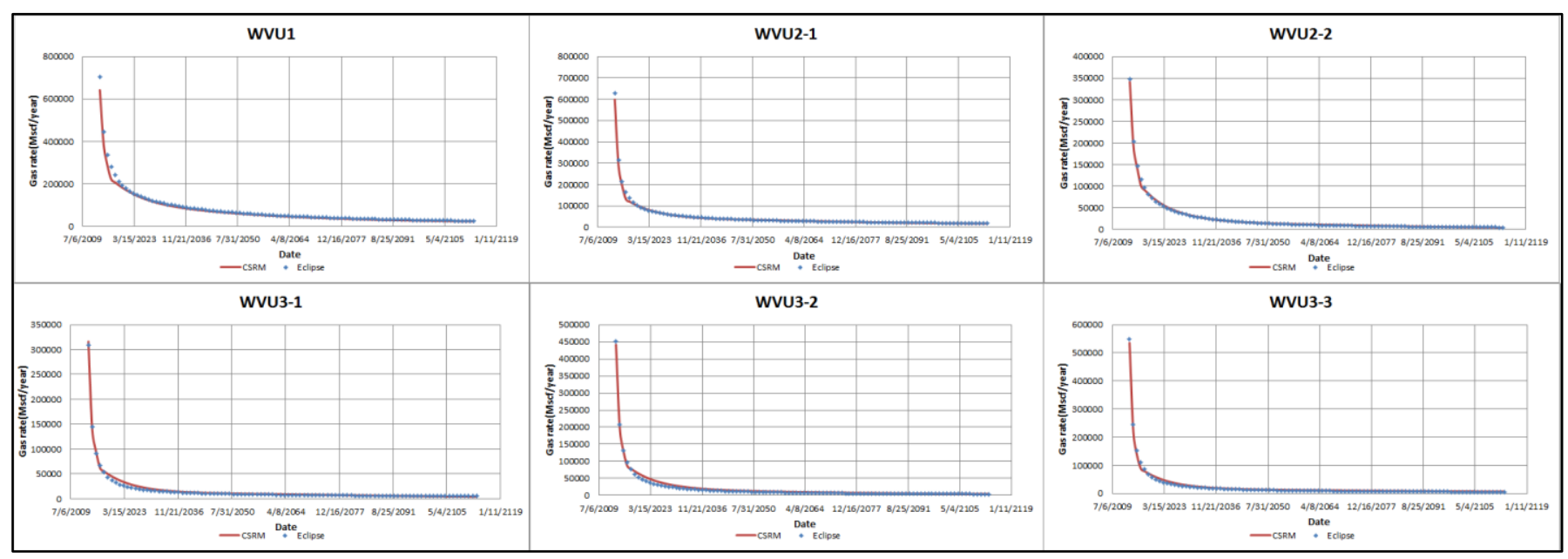

Figure A- 27 Comparison of gas production profile from simulator and Annual Data-driven proxy model-Run10 (Whole lateral) 


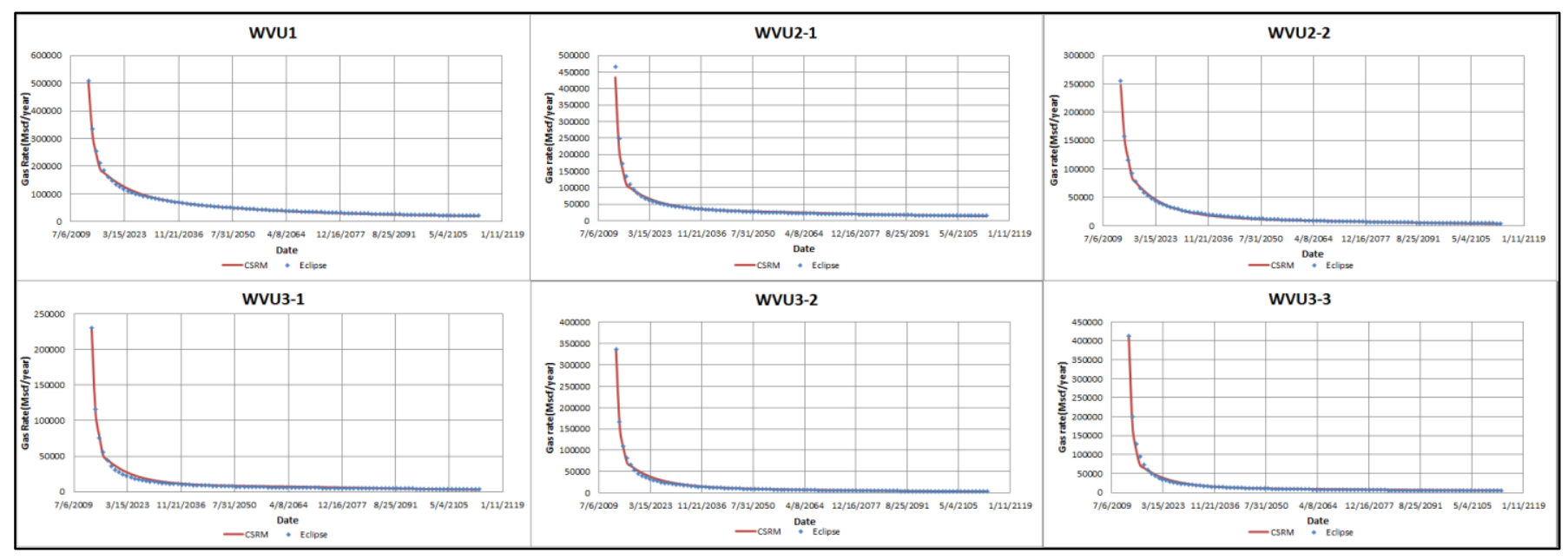

Figure A- 28 Comparison of gas production profile from simulator and Annual Data-driven proxy model-Run10 (Whole lateral)

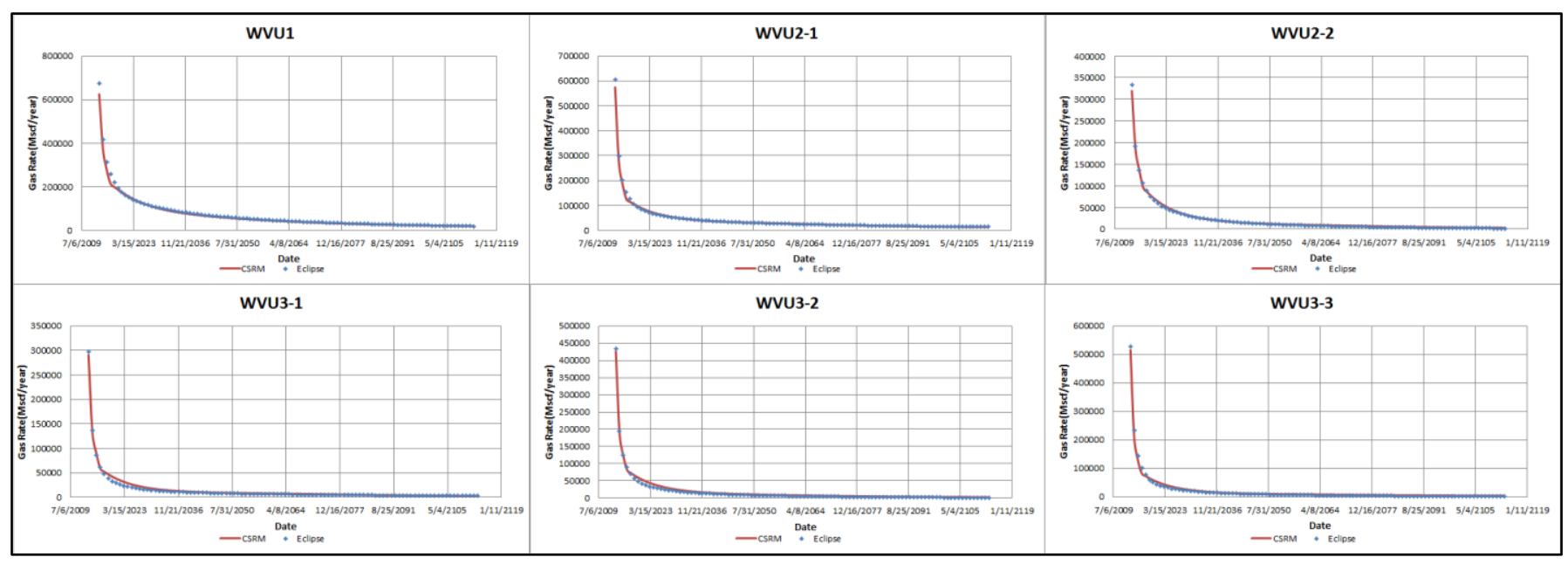

Figure A- 29 Comparison of gas production profile from simulator and Annual Data-driven proxy model-Run10 (Whole lateral)

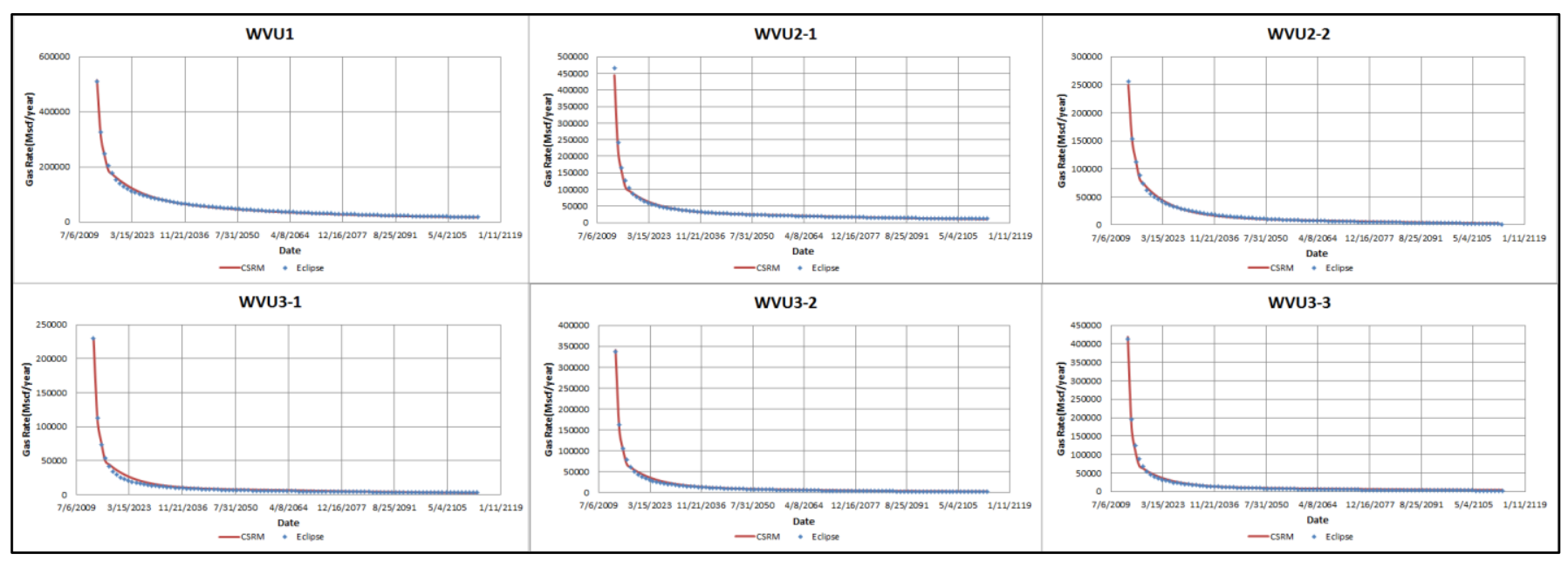

Figure A- 30 Comparison of gas production profile from simulator and Annual Data-driven proxy model-Run10 (Whole lateral) 


\section{APPENDIX B-COMPARISON OF $\mathrm{CH}_{4}, \mathrm{CO}_{2}$ PRODUCTION AND $\mathrm{CO}_{2}$ INJECTION RATES GENEARTED BY DATA-DRIVEN $\mathrm{CO}_{2}$-EGR\&SPROXY MODELS WITH THE NUMERICAL SIMULATION RESULTS}

In all the plots, blue dots represent the rates generated by Eclipse and the solid red line is the result of Data-driven $\mathrm{CO}_{2}$-EGR\&S proxy model. The first five runs (Run1 to 5) are corresponding to the Case1, the second five runes (Run 6 to 10) are corresponding to the Case2, the third five runs (Run11 to 15) are corresponding to the Case3 and the fourth five runs (Run16 to 20) are corresponding to the Case4.

There are four plots in each figure to compare the Data-driven proxy model results for $\mathrm{CH}_{4}$ Production (top) $\mathrm{CO}_{2}$ production (bottom left) and $\mathrm{CO}_{2}$ injection (bottom right) with numerical simulation results.

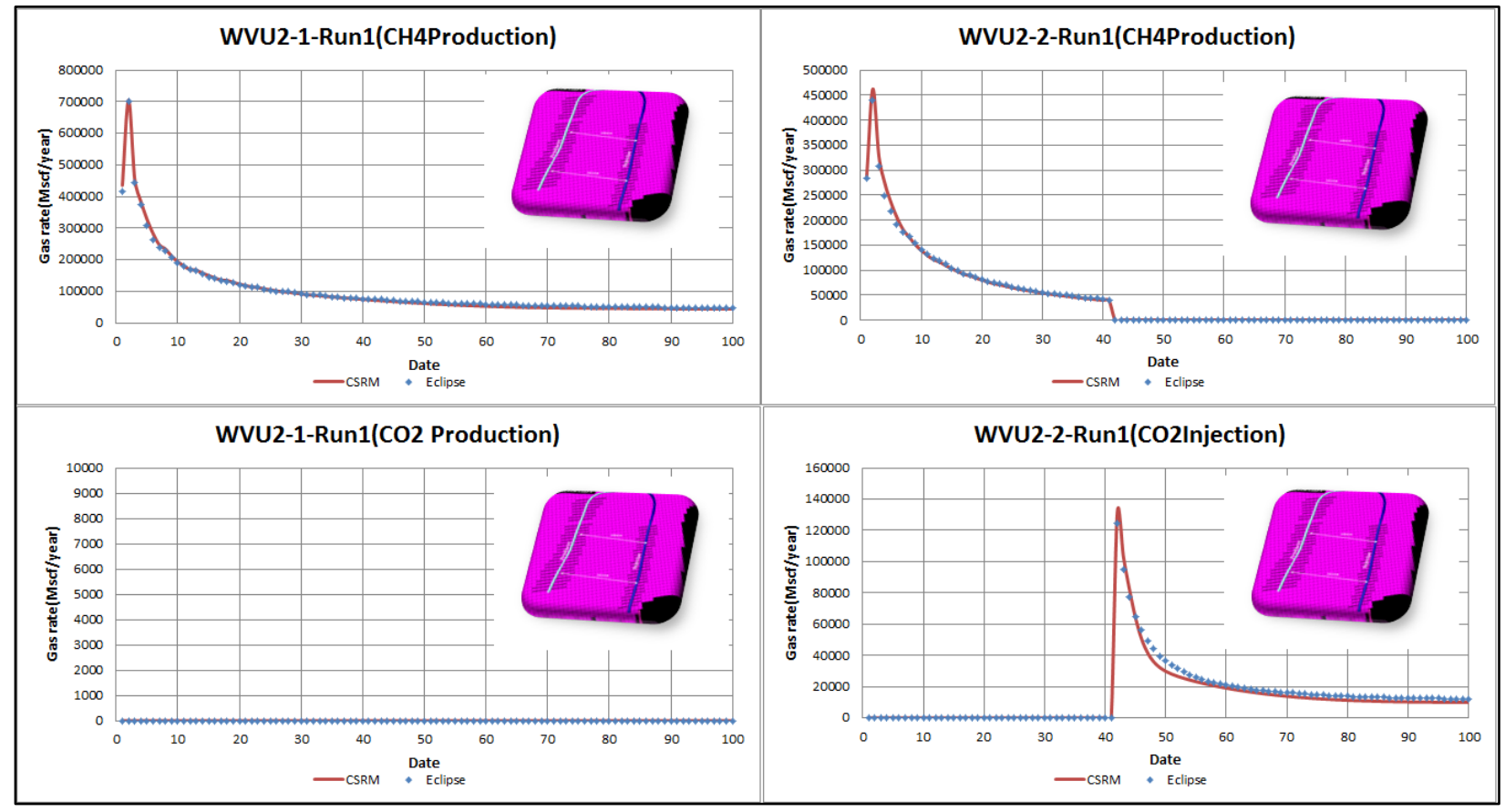

Figure B- 1-Comparison of $\mathrm{CH}_{4}, \mathrm{CO}_{2}$ Production rates (Mscf/year) and $\mathrm{CO}_{2}$ injection rate from simulator and $\mathrm{CO}_{2}$-EGR\&S Datadriven proxy model for producer/injector pairs of laterals (Cases1, Run1) 


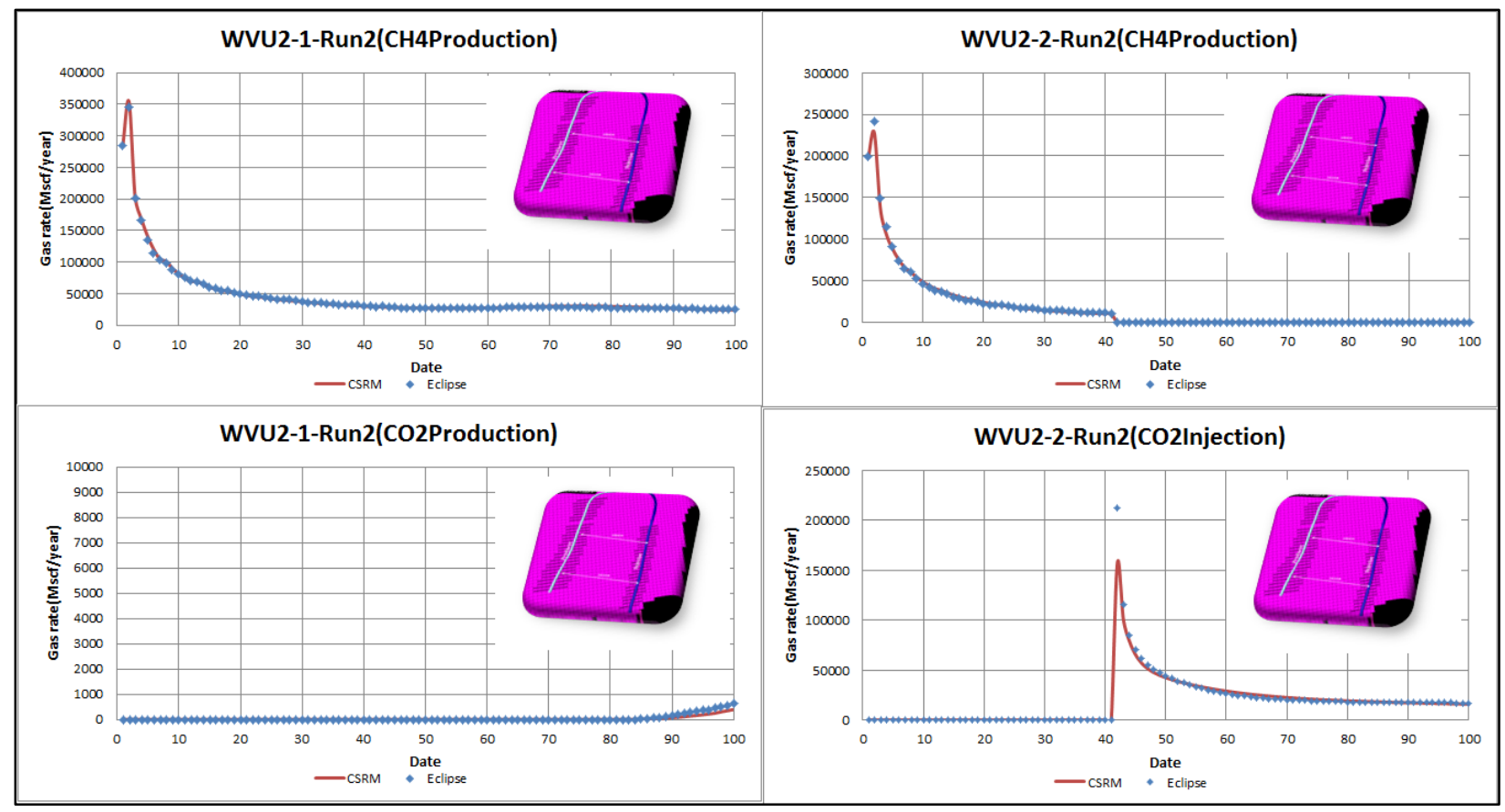

Figure B- 2-Comparison of $\mathrm{CH}_{4}, \mathrm{CO}_{2}$ Production rates (Mscf/year) and $\mathrm{CO}_{2}$ injection rate from simulator and $\mathrm{CO}_{2}$-EGR\&S Datadriven proxy model for producer/injector pairs of laterals (Cases1, Run2)

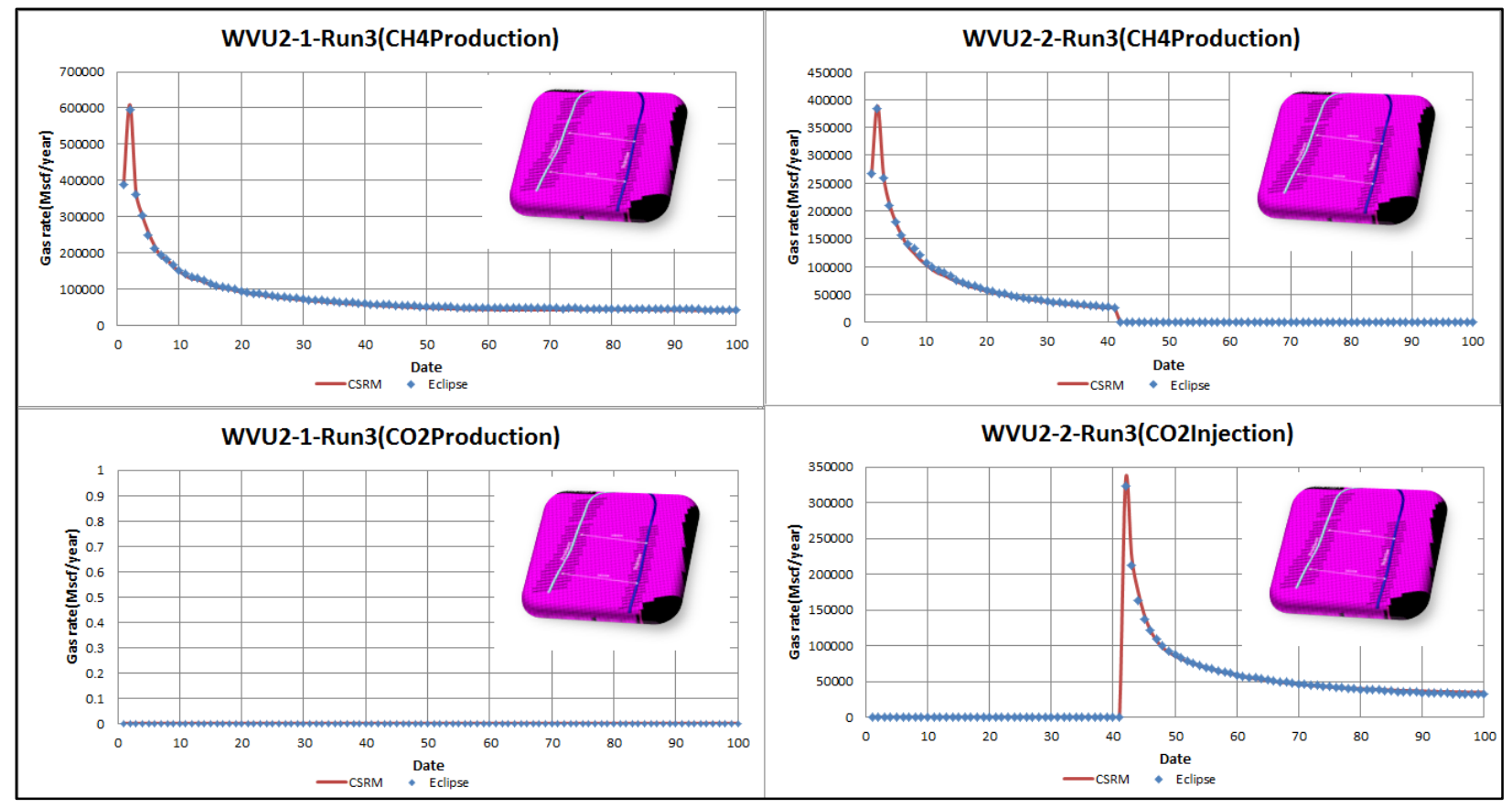

Figure B- 3-Comparison of $\mathrm{CH}_{4}, \mathrm{CO}_{2}$ Production rates (Mscf/year) and $\mathrm{CO}_{2}$ injection rate from simulator and $\mathrm{CO}_{2}$-EGR\&S Datadriven proxy model for producer/injector pairs of laterals (Cases1, Run3) 


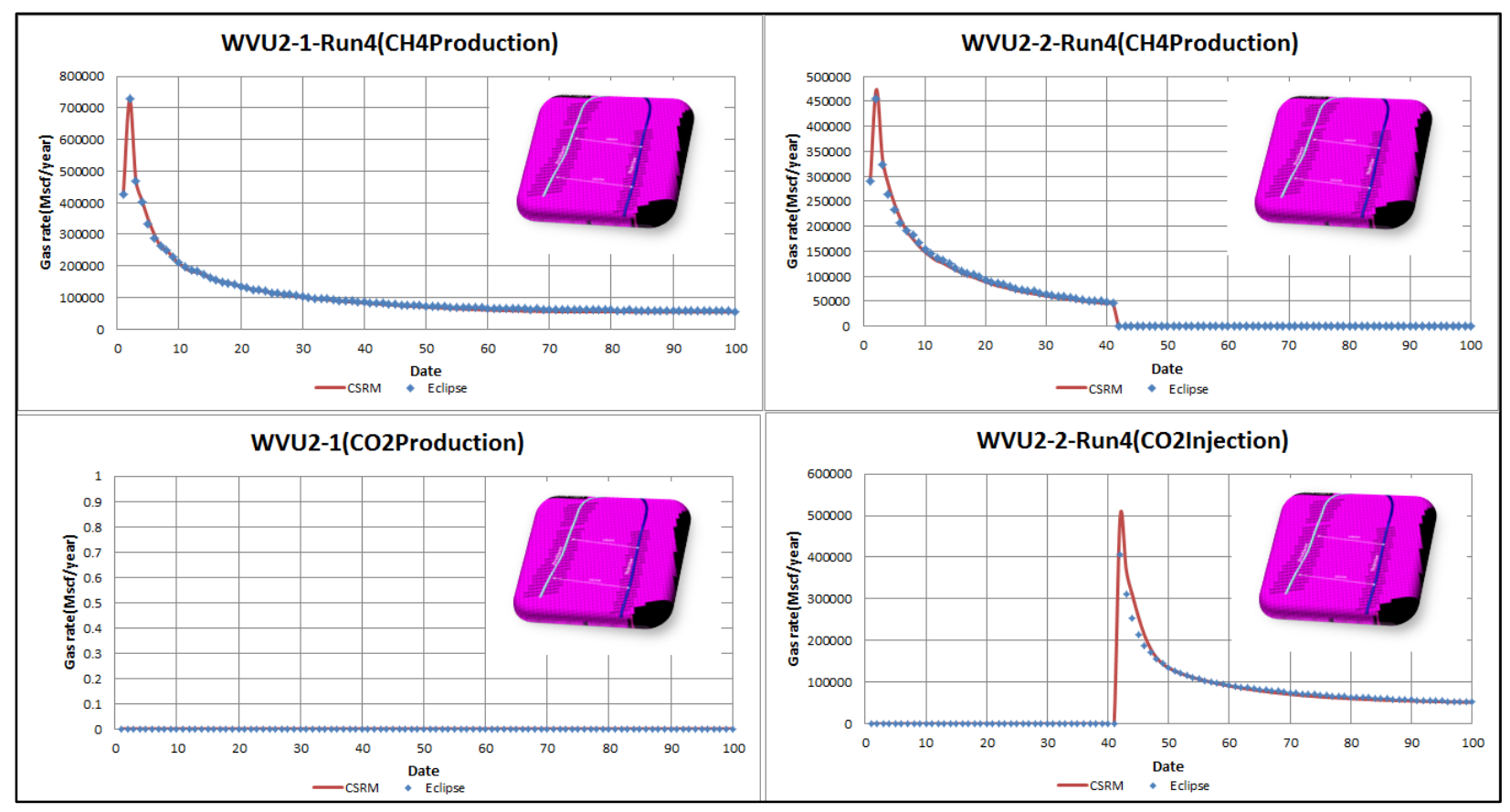

Figure B- 4-Comparison of $\mathrm{CH}_{4}, \mathrm{CO}_{2}$ Production rates (Mscf/year) and $\mathrm{CO}_{2}$ injection rate from simulator and $\mathrm{CO}_{2}$-EGR\&S Datadriven proxy model for producer/injector pairs of laterals (Cases1, Run4)

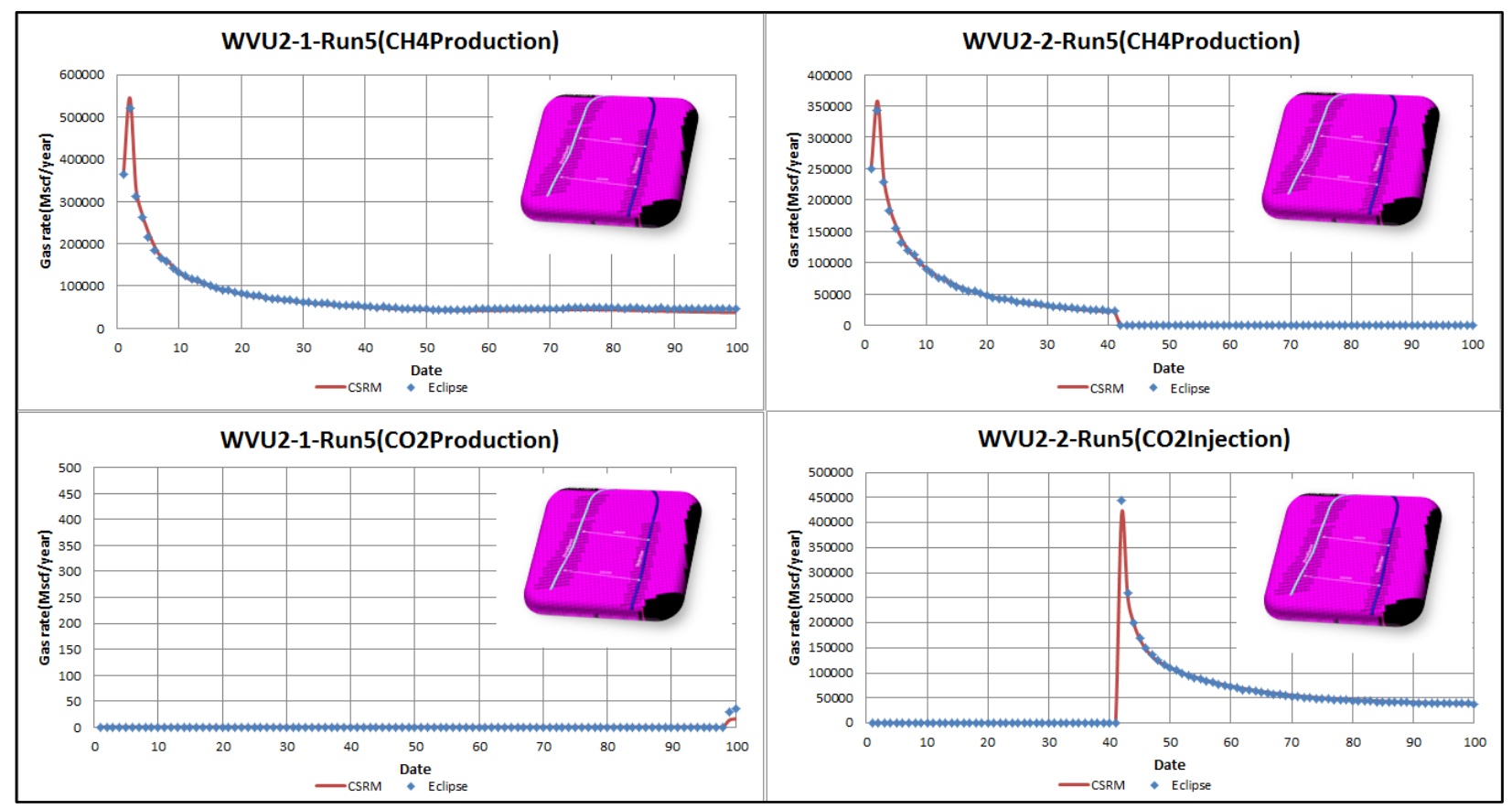

Figure B- 5-Comparison of $\mathrm{CH}_{4}, \mathrm{CO}_{2}$ Production rates (Mscf/year) and $\mathrm{CO}_{2}$ injection rate from simulator and $\mathrm{CO}_{2}$-EGR\&S Datadriven proxy model for producer/injector pairs of laterals (Cases1, Run5) 


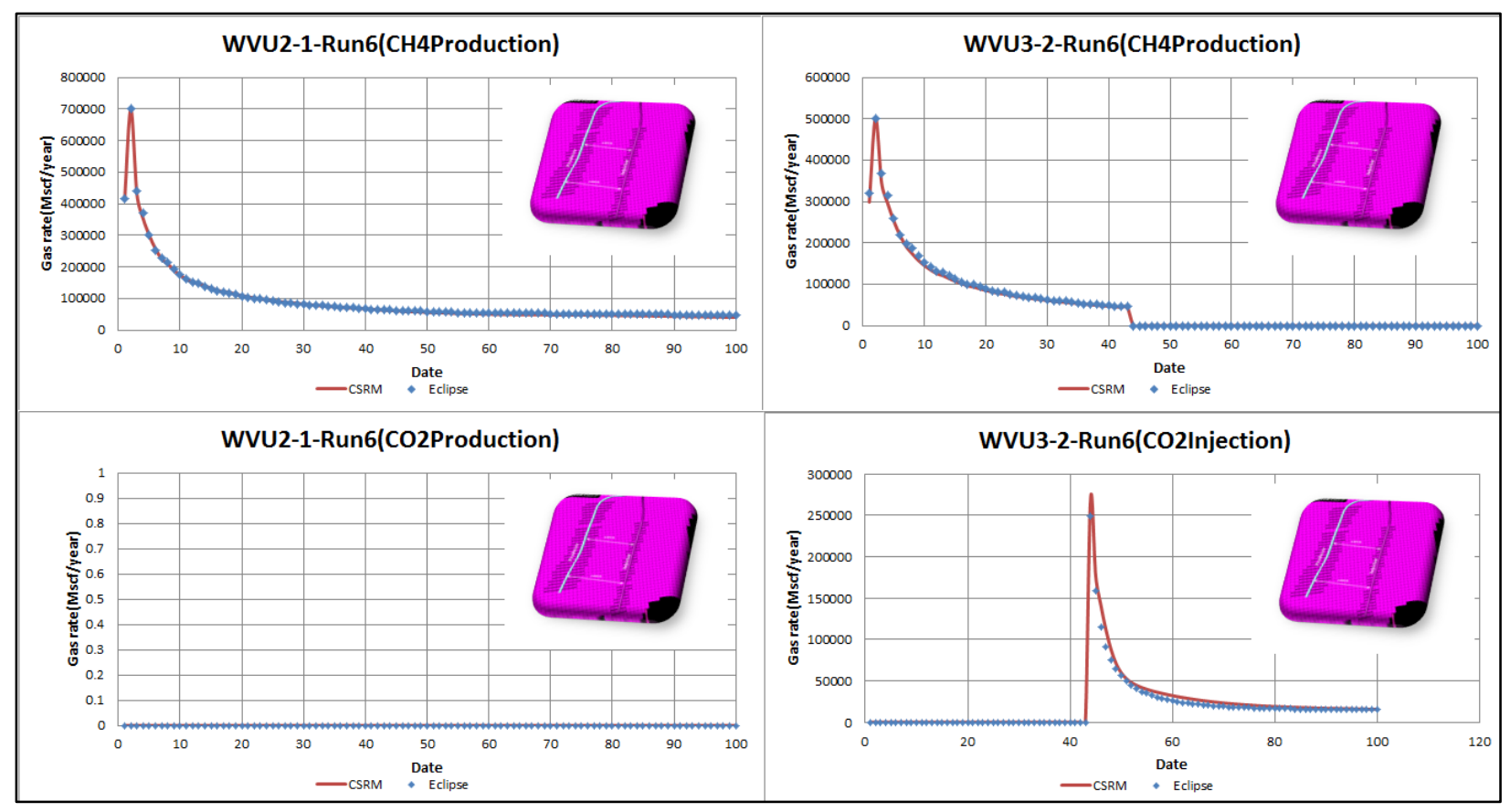

Figure B- 6-Comparison of $\mathrm{CH}_{4}, \mathrm{CO}_{2}$ Production rates (Mscf/year) and $\mathrm{CO}_{2}$ injection rate from simulator and $\mathrm{CO}_{2}$-EGR\&S Datadriven proxy model for producer/injector pairs of laterals (Cases2, Run6)

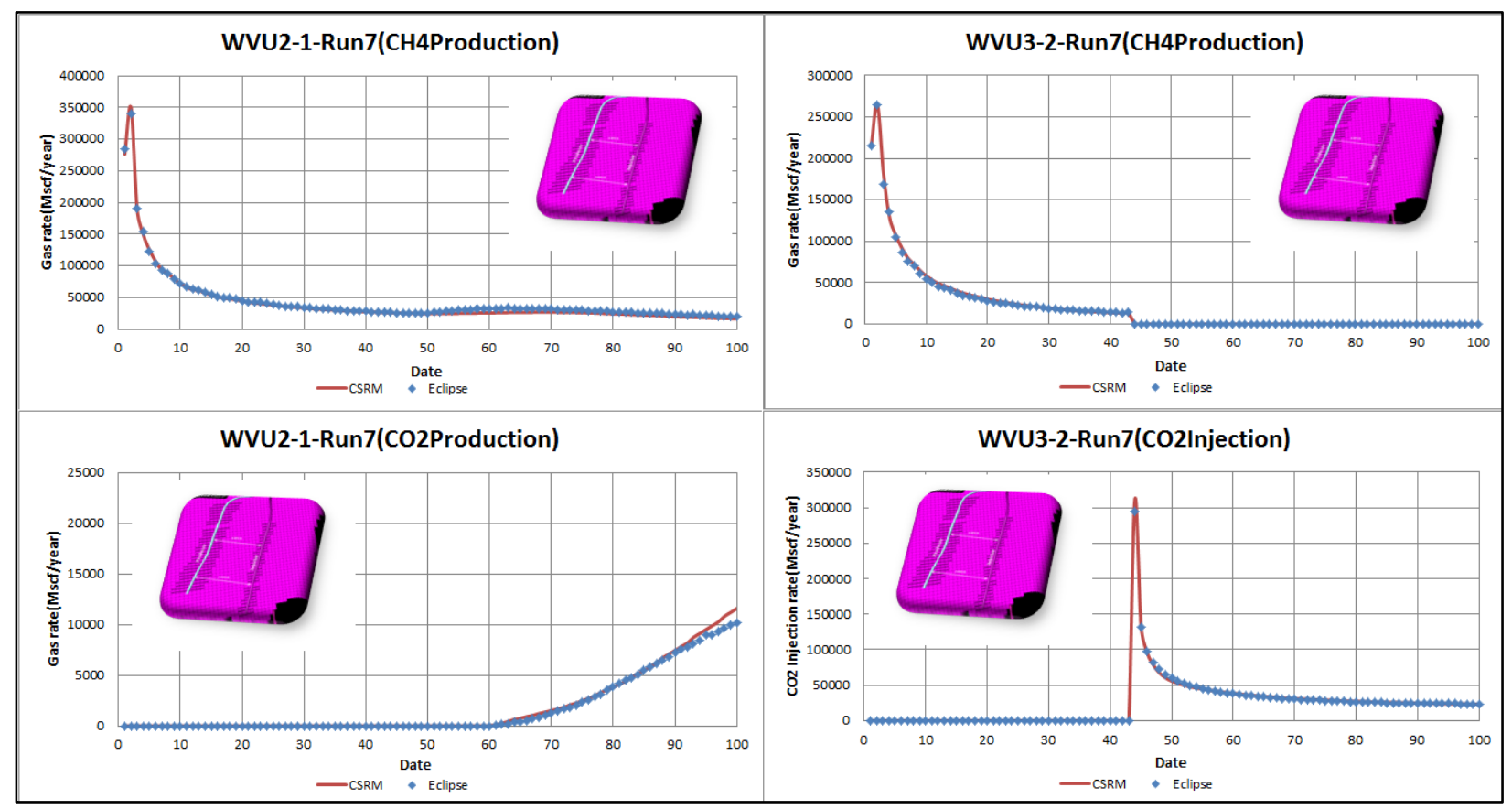

Figure B- 7-Comparison of $\mathrm{CH}_{4}, \mathrm{CO}_{2}$ Production rates (Mscf/year) and $\mathrm{CO}_{2}$ injection rate from simulator and $\mathrm{CO}_{2}$-EGR\&S Datadriven proxy model for producer/injector pairs of laterals (Cases2, Run7) 


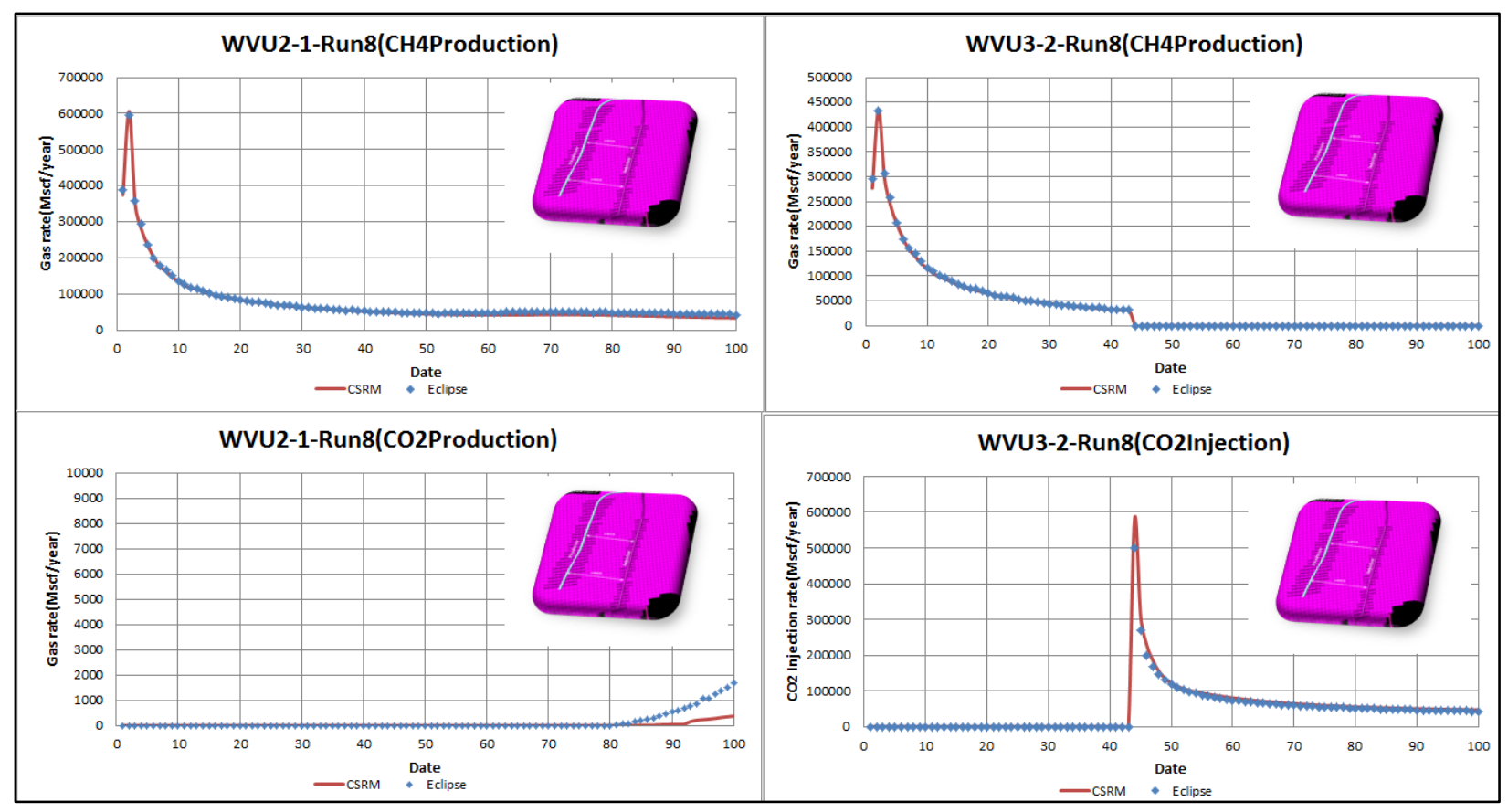

Figure B- 8-Comparison of $\mathrm{CH}_{4}, \mathrm{CO}_{2}$ Production rates (Mscf/year) and $\mathrm{CO}_{2}$ injection rate from simulator and $\mathrm{CO}_{2}$-EGR\&S Datadriven proxy model for producer/injector pairs of laterals (Cases2, Run8)

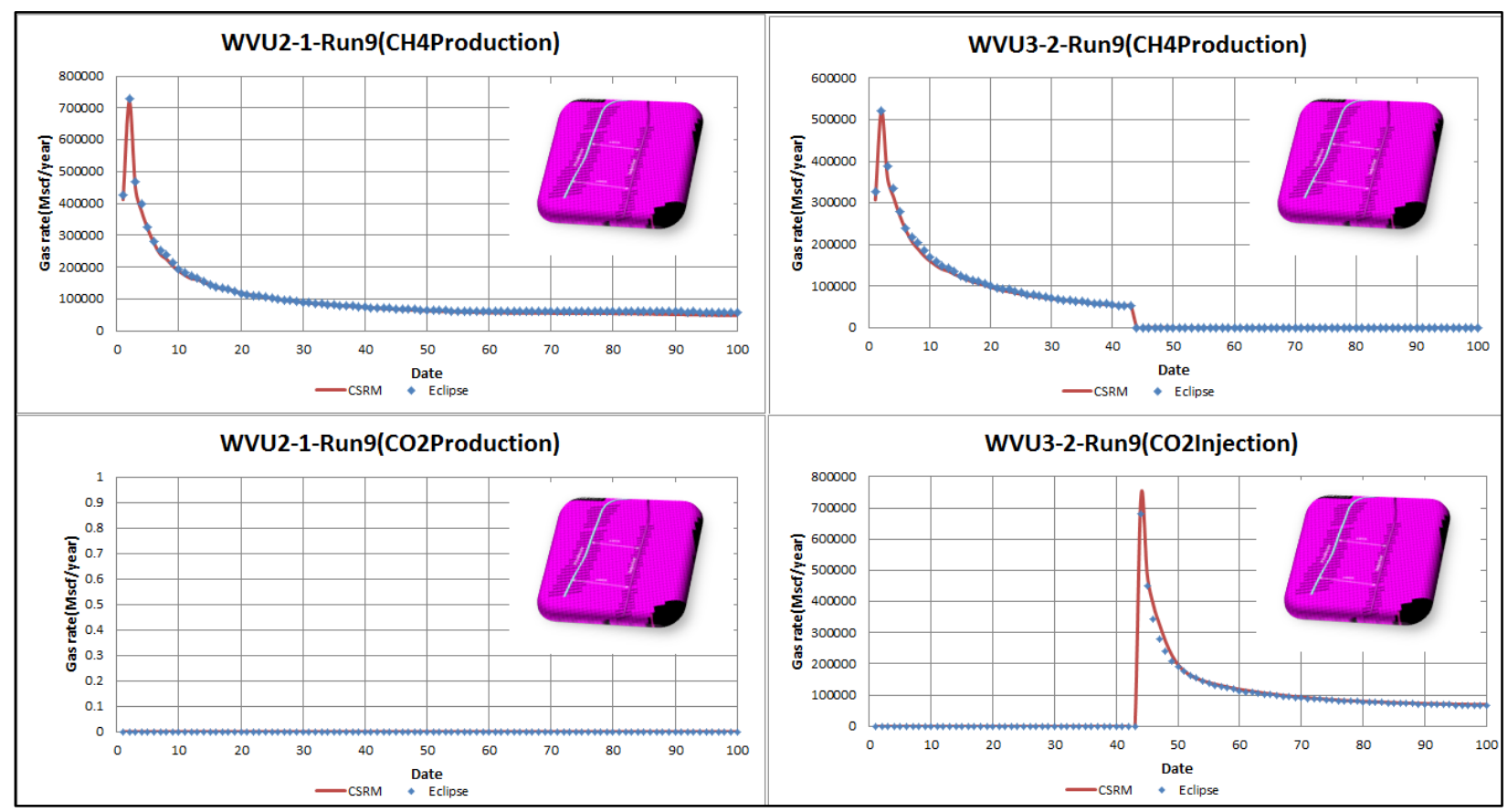

Figure B- 9-Comparison of $\mathrm{CH}_{4}, \mathrm{CO}_{2}$ Production rates (Mscf/year) and $\mathrm{CO}_{2}$ injection rate from simulator and $\mathrm{CO}_{2}$-EGR\&S Datadriven proxy model for producer/injector pairs of laterals (Cases2, Run9) 


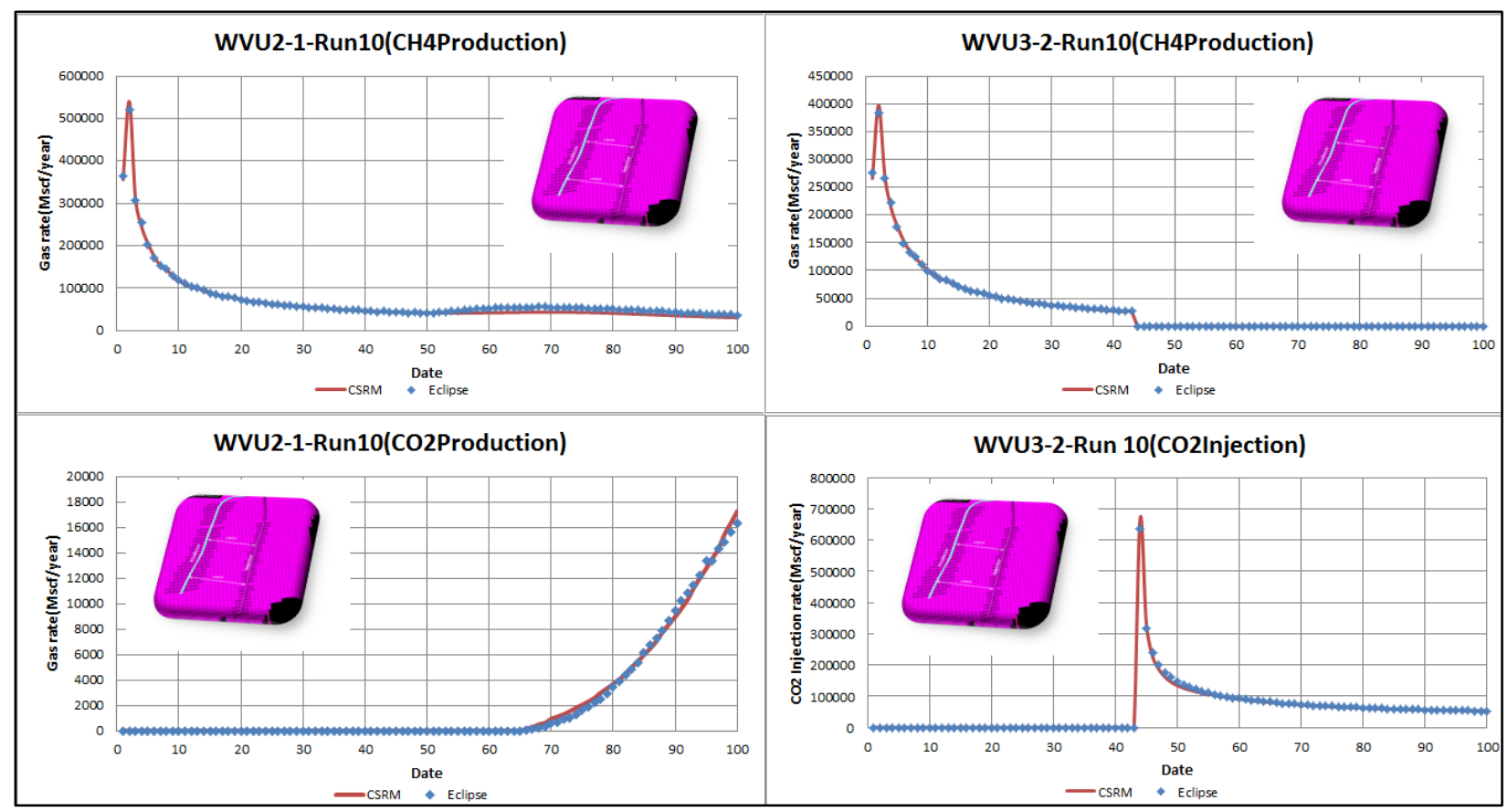

Figure B- 10-Comparison of $\mathrm{CH}_{4}, \mathrm{CO}_{2}$ Production rates (Mscf/year) and $\mathrm{CO}_{2}$ injection rate from simulator and $\mathrm{CO}_{2}$-EGR\&S Data-driven proxy model for producer/injector pairs of laterals (Cases2, Run10)

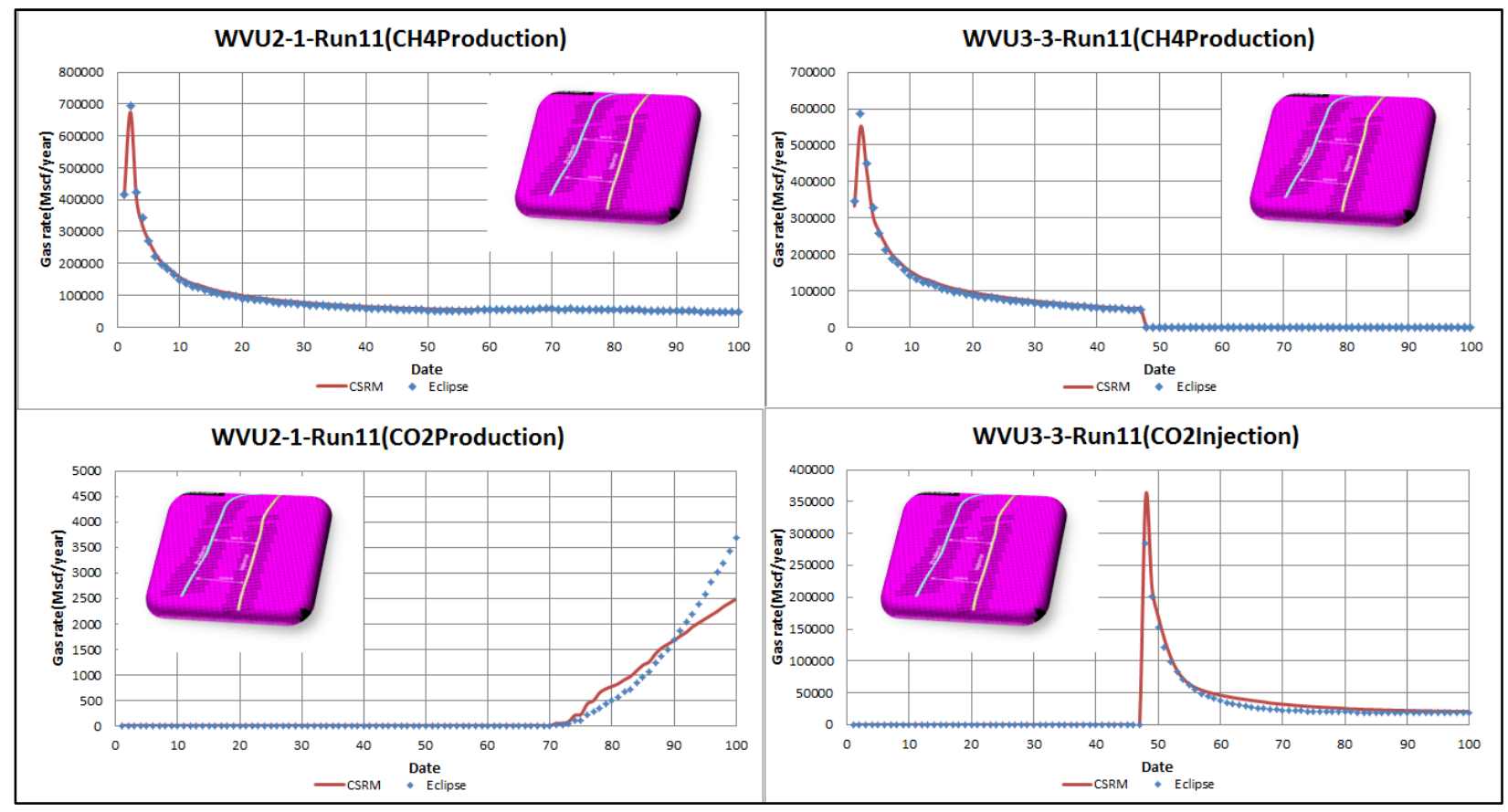

Figure B- 11-Comparison of $\mathrm{CH}_{4}, \mathrm{CO}_{2}$ Production rates (Mscf/year) and $\mathrm{CO}_{2}$ injection rate from simulator and $\mathrm{CO}_{2}$-EGR\&S Data-driven proxy model for producer/injector pairs of laterals (Cases3, Run11) 


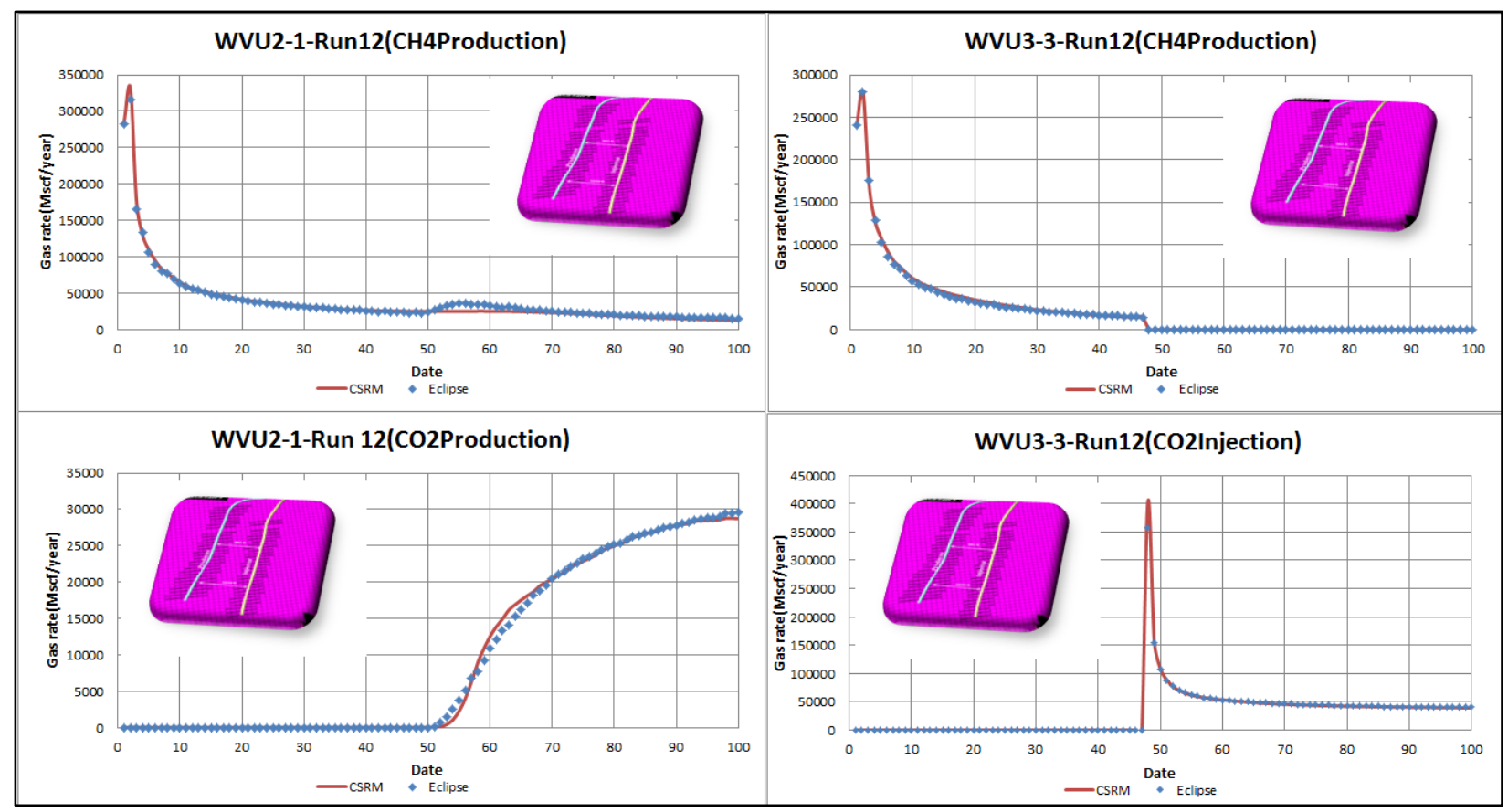

Figure B- 12-Comparison of $\mathrm{CH}_{4}, \mathrm{CO}_{2}$ Production rates (Mscf/year) and $\mathrm{CO}_{2}$ injection rate from simulator and $\mathrm{CO}_{2}$-EGR\&S Data-driven proxy model for producer/injector pairs of laterals (Cases3, Run12)

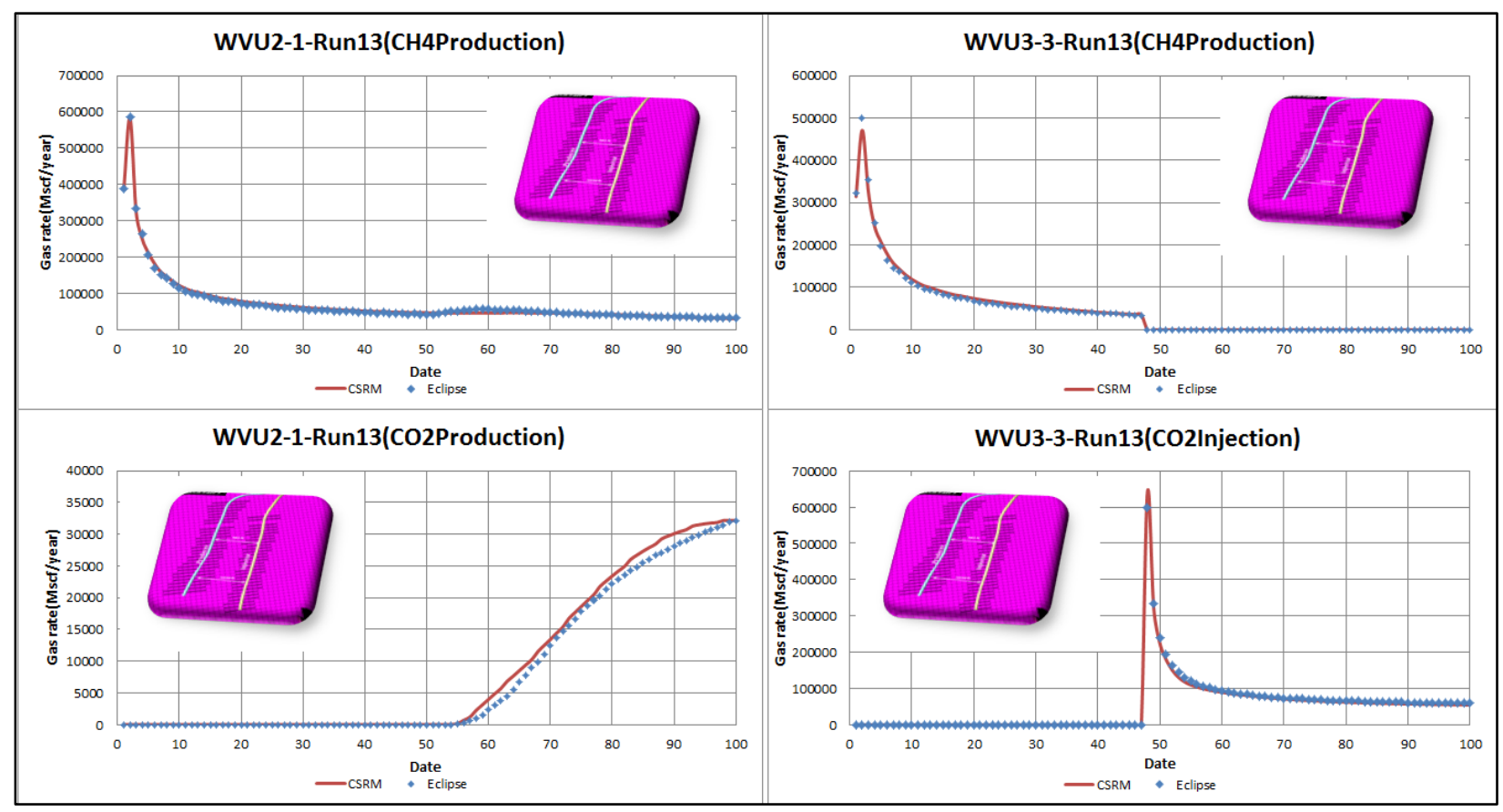

Figure B- 13-Comparison of $\mathrm{CH}_{4}, \mathrm{CO}_{2}$ Production rates (Mscf/year) and $\mathrm{CO}_{2}$ injection rate from simulator and $\mathrm{CO}_{2}$-EGR\&S Data-driven proxy model for producer/injector pairs of laterals (Cases3, Run13) 


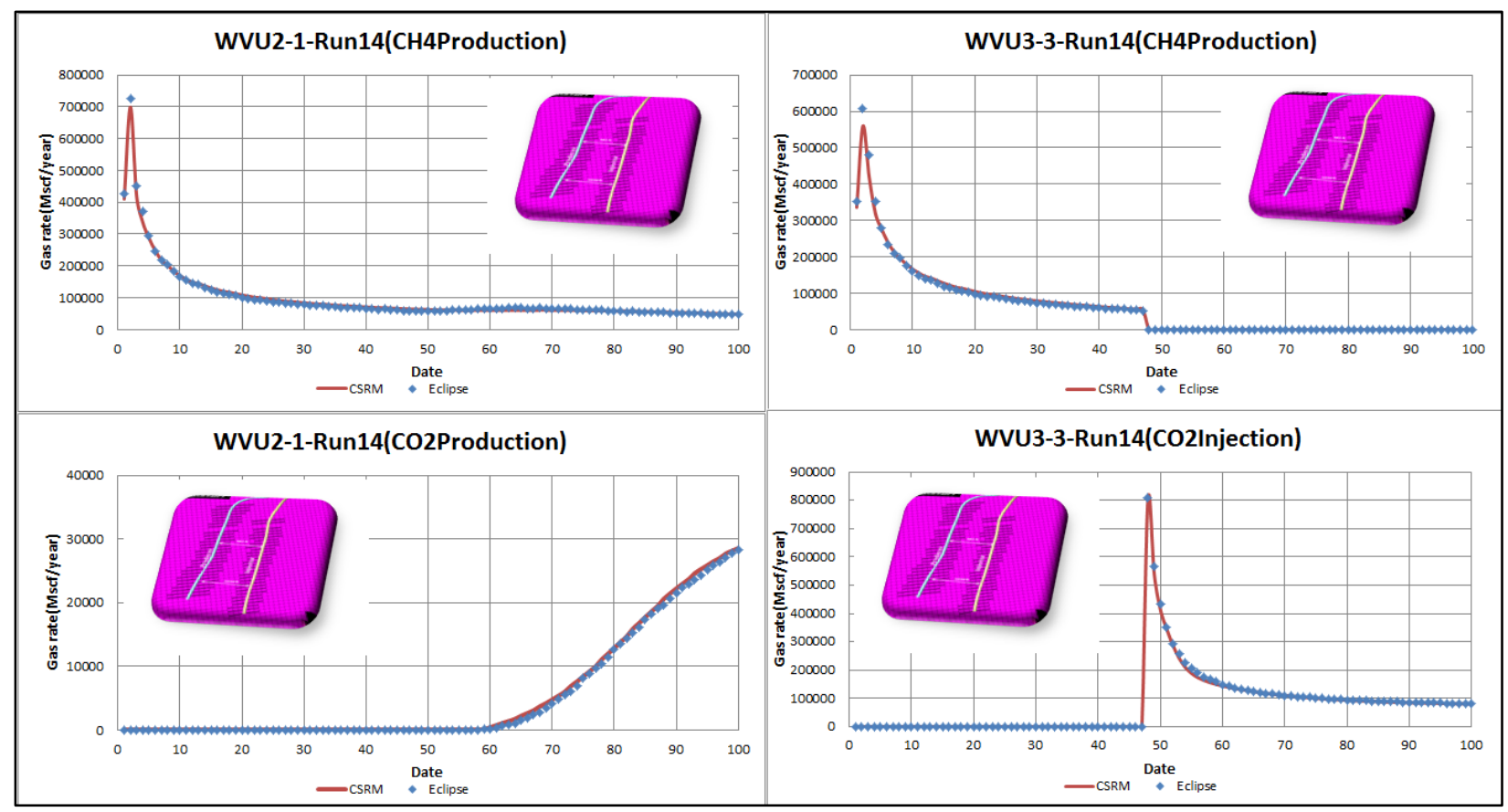

Figure B- 14-Comparison of $\mathrm{CH}_{4}, \mathrm{CO}_{2}$ Production rates (Mscf/year) and $\mathrm{CO}_{2}$ injection rate from simulator and $\mathrm{CO}_{2}$-EGR\&S Data-driven proxy model for producer/injector pairs of laterals (Cases3, Run14)

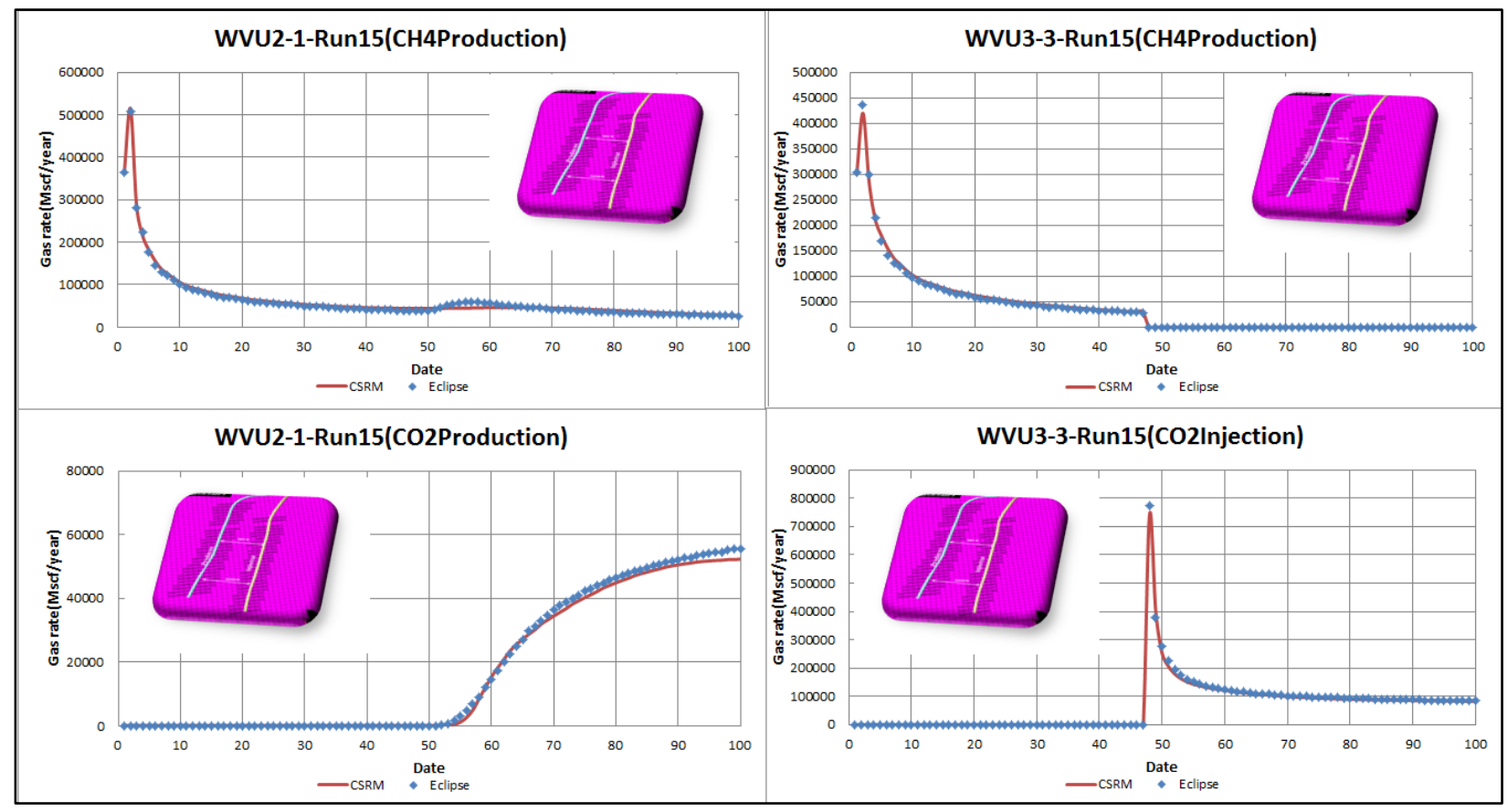

Figure B- 15-Comparison of $\mathrm{CH}_{4}, \mathrm{CO}_{2}$ Production rates (Mscf/year) and $\mathrm{CO}_{2}$ injection rate from simulator and $\mathrm{CO}_{2}$-EGR\&S Data-driven proxy model for producer/injector pairs of laterals (Cases3, Run15) 


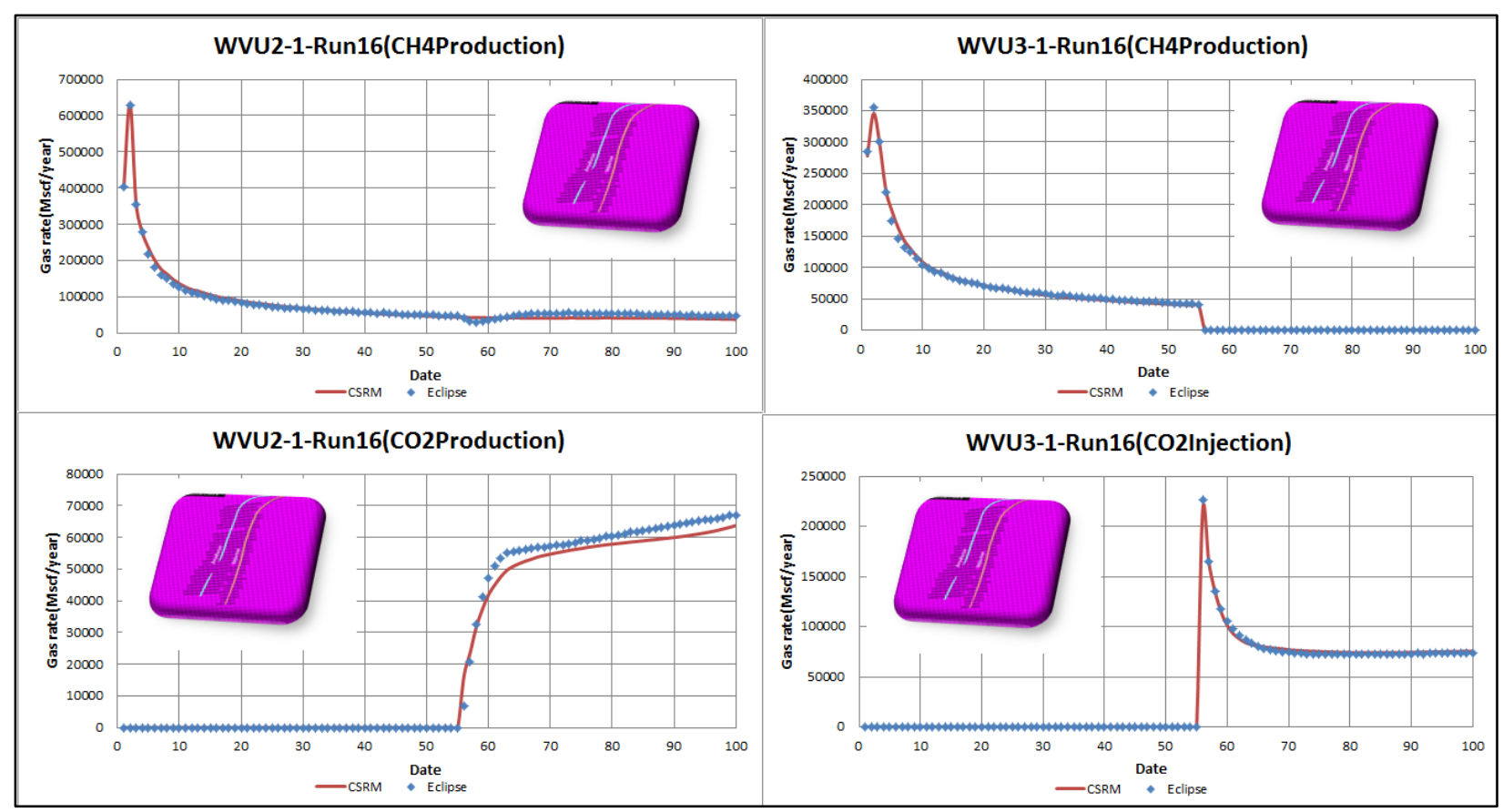

Figure B- 16-Comparison of $\mathrm{CH}_{4}, \mathrm{CO}_{2}$ Production rates (Mscf/year) and $\mathrm{CO}_{2}$ injection rate from simulator and $\mathrm{CO}_{2}$-EGR\&S Data-driven proxy model for producer/injector pairs of laterals (Cases4, Run16)

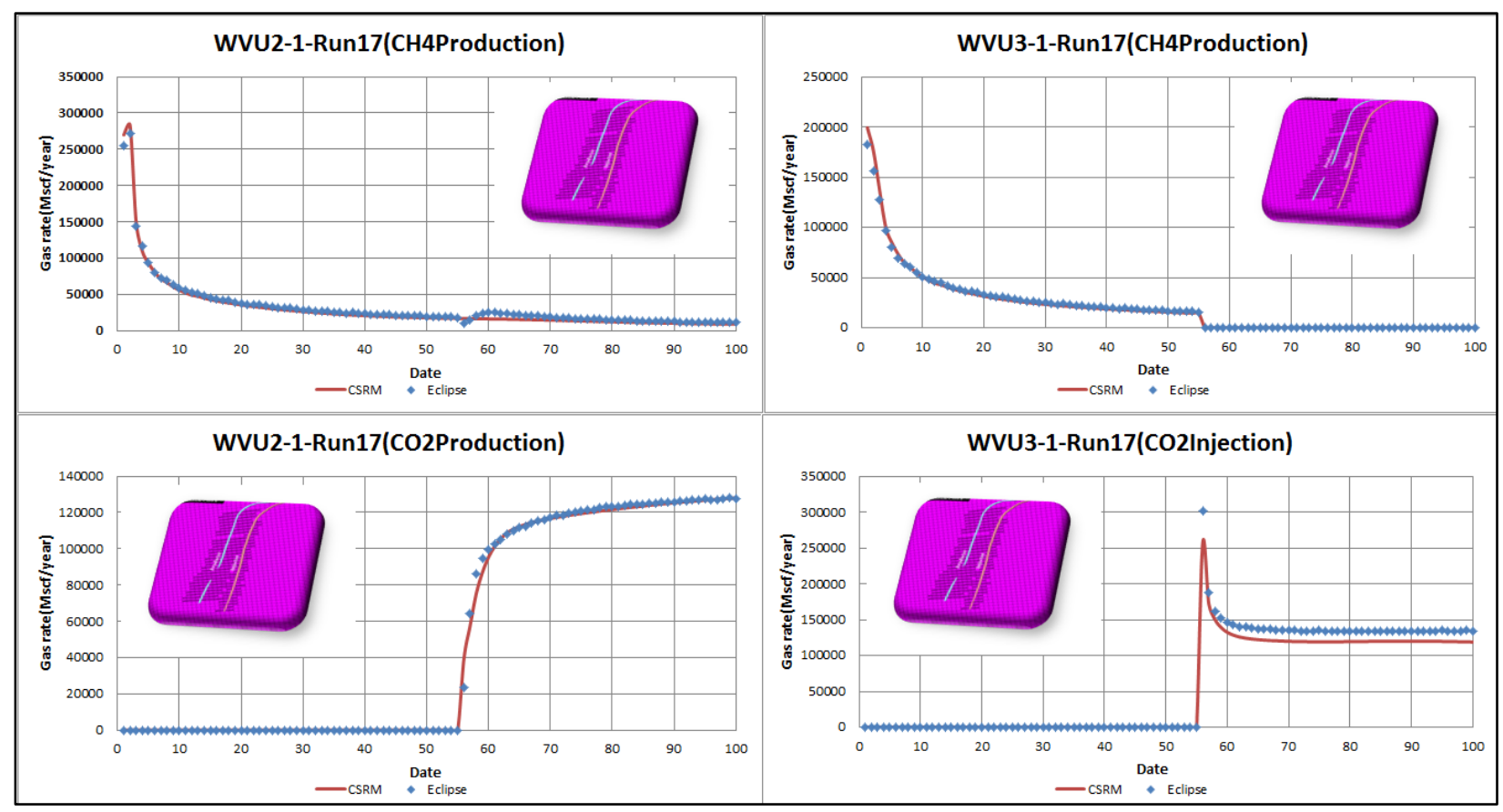

Figure B- 17-Comparison of $\mathrm{CH}_{4}, \mathrm{CO}_{2}$ Production rates (Mscf/year) and $\mathrm{CO}_{2}$ injection rate from simulator and $\mathrm{CO}_{2}$-EGR\&S Data-driven proxy model for producer/injector pairs of laterals (Cases4, Run17) 


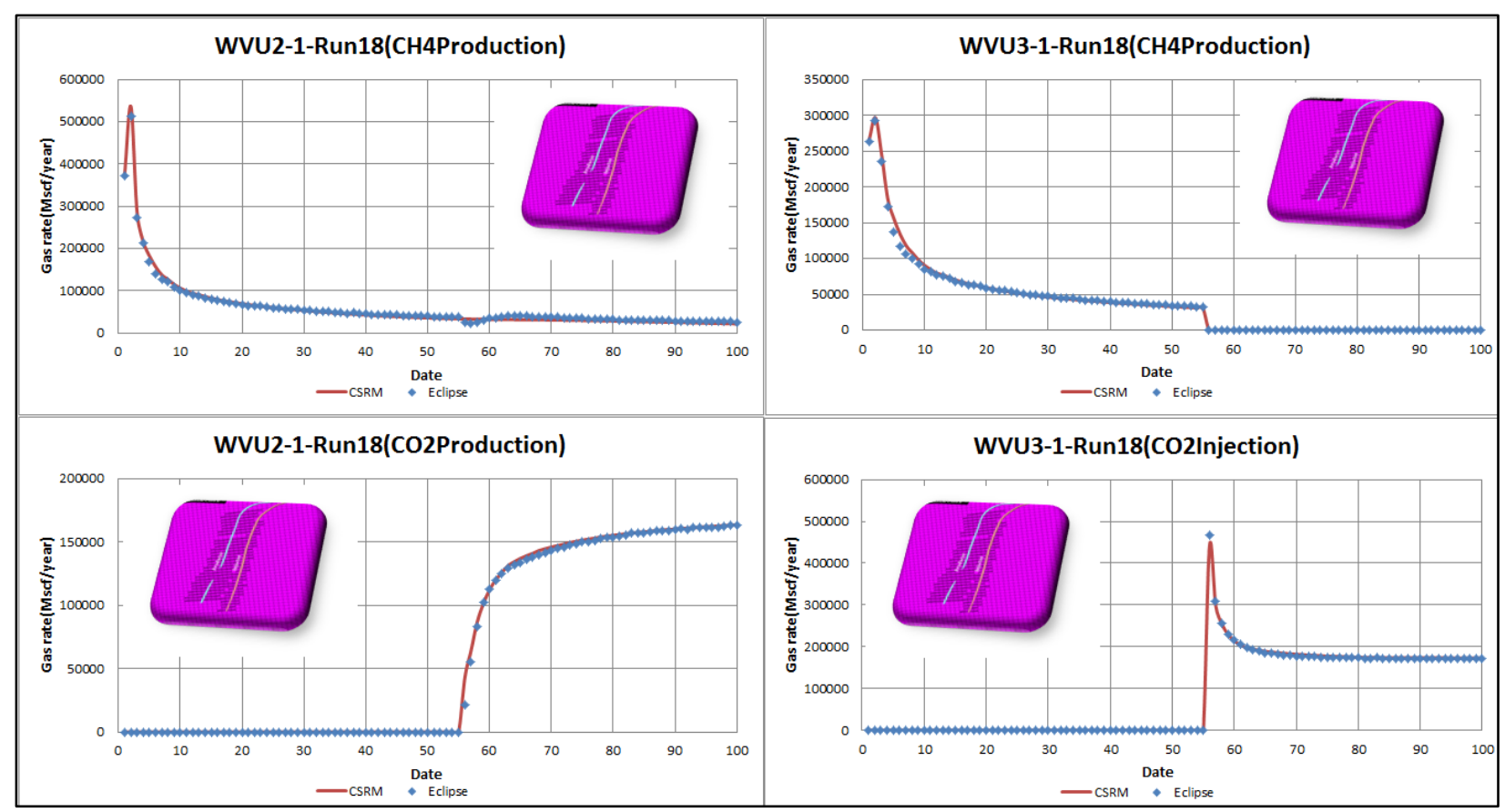

Figure B- 18-Comparison of $\mathrm{CH}_{4}, \mathrm{CO}_{2}$ Production rates (Mscf/year) and $\mathrm{CO}_{2}$ injection rate from simulator and $\mathrm{CO}_{2}$-EGR\&S Data-driven proxy model for producer/injector pairs of laterals (Cases4, Run18)

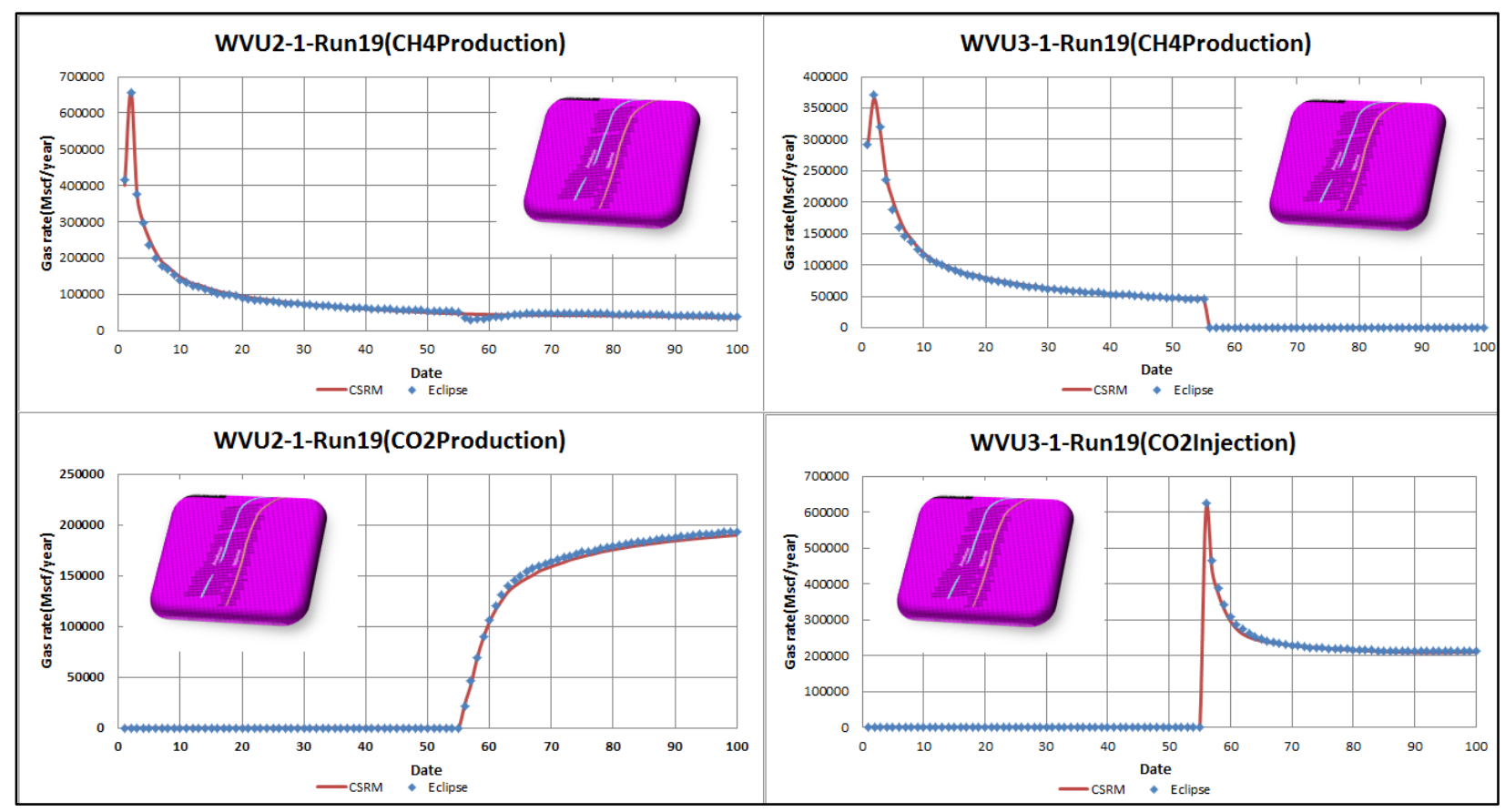

Figure B- 19-Comparison of $\mathrm{CH}_{4}, \mathrm{CO}_{2}$ Production rates (Mscf/year) and $\mathrm{CO}_{2}$ injection rate from simulator and $\mathrm{CO}_{2}$-EGR\&S Data-driven proxy model for producer/injector pairs of laterals (Cases4, Run19) 


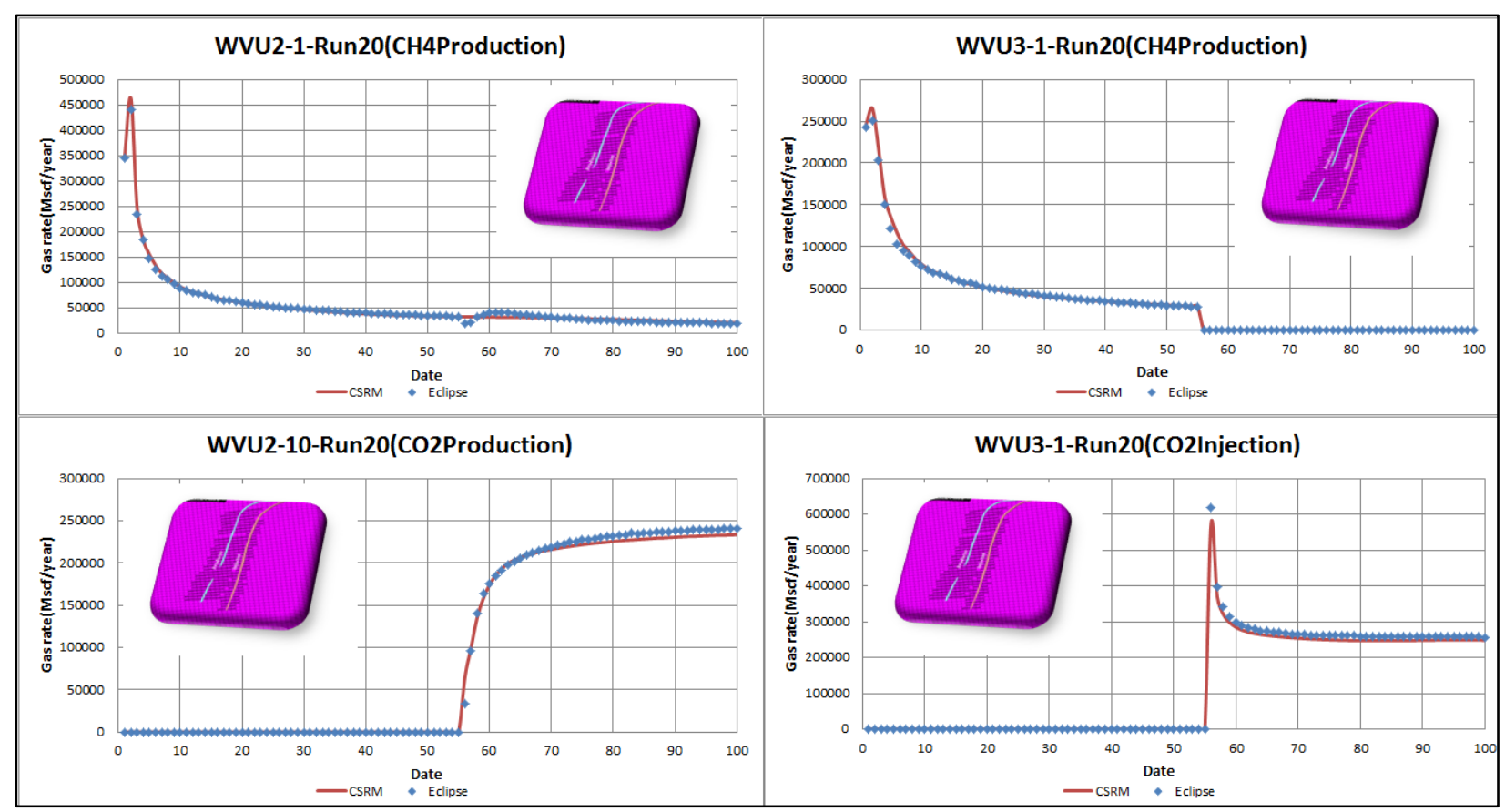

Figure B- 20-Comparison of $\mathrm{CH}_{4}, \mathrm{CO}_{2}$ Production rates (Mscf/year) and $\mathrm{CO}_{2}$ injection rate from simulator and $\mathrm{CO}_{2}$-EGR\&S Data-driven proxy model for producer/injector pairs of laterals (Cases4, Run20) 